\title{
Surviving childhood : new perspectives on the link between childhood trauma and psychosis
}

Citation for published version (APA):

van Nierop, M. (2015). Surviving childhood : new perspectives on the link between childhood trauma and psychosis. [Doctoral Thesis, Maastricht University]. Maastricht University. https://doi.org/10.26481/dis.20150130mn

Document status and date:

Published: 01/01/2015

DOI:

10.26481/dis.20150130mn

Document Version:

Publisher's PDF, also known as Version of record

\section{Please check the document version of this publication:}

- A submitted manuscript is the version of the article upon submission and before peer-review. There can be important differences between the submitted version and the official published version of record.

People interested in the research are advised to contact the author for the final version of the publication, or visit the DOI to the publisher's website.

- The final author version and the galley proof are versions of the publication after peer review.

- The final published version features the final layout of the paper including the volume, issue and page numbers.

Link to publication

\footnotetext{
General rights rights.

- You may freely distribute the URL identifying the publication in the public portal. please follow below link for the End User Agreement:

www.umlib.nl/taverne-license

Take down policy

If you believe that this document breaches copyright please contact us at:

repository@maastrichtuniversity.nl

providing details and we will investigate your claim.
}

Copyright and moral rights for the publications made accessible in the public portal are retained by the authors and/or other copyright owners and it is a condition of accessing publications that users recognise and abide by the legal requirements associated with these

- Users may download and print one copy of any publication from the public portal for the purpose of private study or research.

- You may not further distribute the material or use it for any profit-making activity or commercial gain

If the publication is distributed under the terms of Article $25 \mathrm{fa}$ of the Dutch Copyright Act, indicated by the "Taverne" license above, 


\section{SURVIVING CHILDHOOD}

NEW PERSPECTIVES ON THE LINK BETWEEN

CHILDHOOD TRAUMA AND PSYCHOSIS 
(C) M. van Nierop, Maastricht 2014

All rights reserved. No part of this thesis may be reproduced, stored in a retrieval system, or transmitted, in any form or by any means, electronic, mechanical, photocopying, recording, or otherwise, without the prior permission in writing from the proprietor.

\section{Cover design}

Martine van Nierop

\section{Layout}

Formzet BV, Zoetermeer

\section{Print}

Ridderprint BV, Ridderkerk 


\section{SURVIVING CHILDHOOD}

\section{NEW PERSPECTIVES ON THE LINK BETWEEN CHILDHOOD TRAUMA AND PSYCHOSIS}

\section{Proefschrift}

Ter verkrijging van de graad van doctor aan de Universiteit Maastricht, op gezag van de Rector Magnificus, Prof. dr. L.L.G. Soete, volgens het besluit van het College van Decanen, in het openbaar te verdedigen op vrijdag 30 januari 2015, om 12:00 uur

door

Martine van Nierop

(Den Haag, 25 juli 1982) 


\section{Promotoren}

Prof. dr. I. Myin-Germeys

Prof. dr. J. van Os

\section{Co-promotor}

Dr. R. van Winkel

\section{Beoordelingscommissie}

Prof. dr. P. Delespaul (voorzitter)

Prof. dr. M. van der Gaag (VU Amsterdam)

Prof. dr. F. Peeters

Prof. dr. J.P. Selten

Dr. W. Veling (University Medical Center Groningen)

The research presented in this thesis was performed at the School for Mental Health \& Neuroscience (MHeNS), department of Psychiatry \& Psychology, Maastricht University, Maastricht, the Netherlands

The publication of this thesis was financially supported by: Formzet B.V. te Zoetermeer 


\section{Don't panic \\ (Douglas Adams)}




\section{Paranimfen}

Rufa Diederen

Claudia Menne-Lothmann 


\section{Table of contents}

CHAPTER 1

Introduction

\section{CHAPTER 2}

Phenotypically Continuous with Clinical Psychosis, Discontinuous in Need for Care:

Evidence for an Extended Psychosis Phenotype

\section{CHAPTER 3}

Evidence That Transition from Health to Psychotic Disorder Can Be Traced to SemiUbiquitous Environmental Effects Operating against Background Genetic Risk

\section{CHAPTER 4}

Psychopathological Mechanisms Linking Childhood Traumatic Experiences to Risk of Psychotic Symptoms: Analysis of a Large, Representative Population-Based Sample

\section{CHAPTER 5}

Childhood Trauma as a Cause of Psychosis: Linking Genes, Psychology and Biology

\section{CHAPTER 6}

Does Social Defeat Mediate the Association Between Childhood Trauma and Psychosis? Evidence from the Nemesis-2 Study

\section{CHAPTER 7}

Childhood Trauma and Polygenic Risk for Schizophrenia: No Evidence for Gene-

Environment Correlation or Gene-Environment Interaction in a Family-Based Sample

\section{CHAPTER 8}

Childhood Abuse and Neglect in Relation to the Presence and Persistence of Psychotic and Depressive Symptomatology

\section{CHAPTER 9}

Evidence that Psychotic Symptoms Are Prevalent in Disorders of Anxiety and Depression, Impacting on Illness Onset, Risk, and Severity - Implications for Diagnosis and Ultra-High Risk Research

Childhood Trauma Is Associated With a Specific Admixture of Affective, Anxiety, and Psychosis Symptoms Cutting Across Traditional Diagnostic Boundaries 
The Functional and Clinical Relevance of Childhood Trauma-Related Admixture of Affective, Anxious, and Psychosis Symptoms: Further Support for a Stratified Medicine Approach in Psychiatry

\section{CHAPTER 12}

Discussion

Summary

Valorization

Dankwoord 
CHAPTER 1

Introduction 


\section{The link between childhood traumatic experiences and psychopathology}

\section{What is childhood trauma?}

In psychiatric research, many different types of childhood trauma are investigated, all of which may, immediately or even many years later, lead to psychological suffering. Some examples are physical abuse (e.g. being hit or kicked), psychological abuse (e.g. being yelled at or unjustly punished), sexual abuse (any unwanted sexual act), emotional neglect (e.g. not having any emotional support), or physical neglect (e.g. not having clean clothes, or enough food). Another example is peer victimization, or bullying, which is receiving more and more attention in recent years. A child may have been traumatized by growing up in a foster care situation, or by being involved in a serious accident or natural disaster, or even in a war situation.

Unfortunately, some of these experiences are not uncommon: within the general population, estimates have been reported of about $18 \%$ for psychological abuse ${ }^{1}, 15 \%$ report to have experienced physical abuse ${ }^{1}, 13 \%$ remember to have been subject to regular bullying ${ }^{2}$, and another $6 \%$ reports having endured sexual abuse ${ }^{1}$. Gender differences exist, for example women report more sexual abuse, whereas men report more physical abuse ${ }^{3}$. It also seems part of a vicious cycle: perpetrators of sexual abuse often have a history of sexual trauma themselves ${ }^{4}$. Types of trauma tend to co-occur within the same individual: victims of sexual abuse are often also physically abused ${ }^{1}$. Many types of trauma can go unnoticed for many years, as the child may not feel secure enough to tell someone about it ${ }^{5}$. Or, maybe even worse, no one believes them ${ }^{6}$.

\section{Psychopathology}

Psychiatric symptoms are common in the general population. Many people, for example, will at some point in their lives experience symptoms of depression, but most of these people will not have severe enough symptoms that they meet diagnostic criteria for a mood disorder (e.g. major depressive disorder, or bipolar disorder). These symptoms, outside the context of a psychiatric disorder, are also called 'non-clinical' symptoms. In the Netherlands, about $53.7 \%$ of the population has experienced symptoms of depression at some point in their lives, while about $20.2 \%$ has had a lifetime mood disorder ${ }^{7}$. The same applies for symptoms of anxiety; $57.4 \%$ of the Dutch population reports lifetime anxiety symptoms, yet $19.6 \%$ meets criteria for an anxiety disorder at some point in their life? 
What is less common knowledge is that this also applies for psychosis. Positive ${ }^{\text {a }}$ psychotic symptoms, the most well known symptoms of psychotic disorders such as schizophrenia, are delusions (e.g. 'someone is out to harm me', or 'I am being followed') and hallucinations (e.g. seeing or hearing things that are not there). These types of symptoms are relatively common in the general population, with lifetime estimates of between 4 and $18 \%^{8}$, compared with a lifetime prevalence of $0.5 \%$ of schizophrenia ${ }^{9}$. The highly different reports of prevalence of psychotic symptoms mostly lie in the methods used in different studies; some studies use self-report measures, while others utilize extensive face-to-face clinical interviews ${ }^{10}$.

Generally speaking, self-report measures for psychotic experiences generate high numbers of false-positives ${ }^{11}$, thus leading to higher estimates in studies using self-report measures than in studies using clinical interviews.

In psychiatric research, some studies focus on the clinical diagnoses (e.g. associations of cannabis use with schizophrenia), where others are interested in the full range of psychopathology, and therefore look at the whole range of symptoms that may be present, clinical or non-clinical. Most studies focus on a particular set of symptoms (e.g. depressive symptoms), or a particular diagnosis (e.g. major depressive disorder). However, comorbidity, or co-occurrence of symptoms (e.g. symptoms of depression in schizophrenia patients) is highly prevalent ${ }^{12}$. Moreover, patients with comorbid psychiatric disorders, or with co-occurring symptoms, tend to display decreased functioning and increased disease severity ${ }^{13}$. This means that this comorbidity is very relevant in clinical practice, and should be investigated more thoroughly.

\section{Childhood trauma and psychopathology}

When studying psychopathology, researchers are very interested in potential causal factors. Knowledge of these factors could not only lead to possible prevention (e.g. getting people to stop smoking after the causal association with lung cancer was found), but it also provides important information on how certain diseases develop, or how our brain in general works. One such factor, which has received much attention in the field of psychiatry, is childhood trauma. Associations between childhood trauma and psychopathology have consistently been found. Not only with post-traumatic stress disorder ${ }^{14}$, but also, amongst others, depression ${ }^{15}$, anxiety disorder ${ }^{16}$ and schizophrenia ${ }^{17}$. However, as schizophrenia has long been viewed as a more 'biological' disorder (i.e. caused by a genetic vulnerability), widespread research into childhood trauma as a risk factor for psychosis is relatively young ${ }^{18}$.

Assessing whether these associations are of a causal nature, however, is particularly difficult to prove. In most studies assessing these associations, adult participants are interviewed on their childhood trauma history.

\footnotetext{
a The term 'positive' means that these symptoms are not experienced by others, whereas 'negative' psychotic symptoms are deficits of normal responses or thought processes, such as apathy or lack of motivation.
} 
When individuals are suffering from psychiatric symptoms, it is possible that that they may unconsciously recall events from their childhood differently than individuals without any psychiatric symptoms. In an attempt to make sense of their current situation, they may over-report traumatic events ${ }^{19}$. This is also known as recall bias. Another possible factor that may influence results lies in individual differences already present long before any psychopathology arises; e.g. people who will develop schizophrenia later in life may already display some odd behaviour at a very young age, making them vulnerable to for example bullying. In this example, the traumatic experiences do not cause schizophrenia, but the vulnerability for the disease causes the traumatic experiences. This is called reverse causality ${ }^{20}$ (Figure 1B). Lastly, a genetic predisposition for a certain disease may both lead to the expression of this disease as well as to an increased risk for exposure to trauma. This means that trauma has no impact on the development of this disease, and findings of an association between trauma and a disease are based on so-called geneenvironment correlation ${ }^{21}$ (Figure 1C).

Figure 1 Etiological models explaining the association between childhood trauma and psychopathology

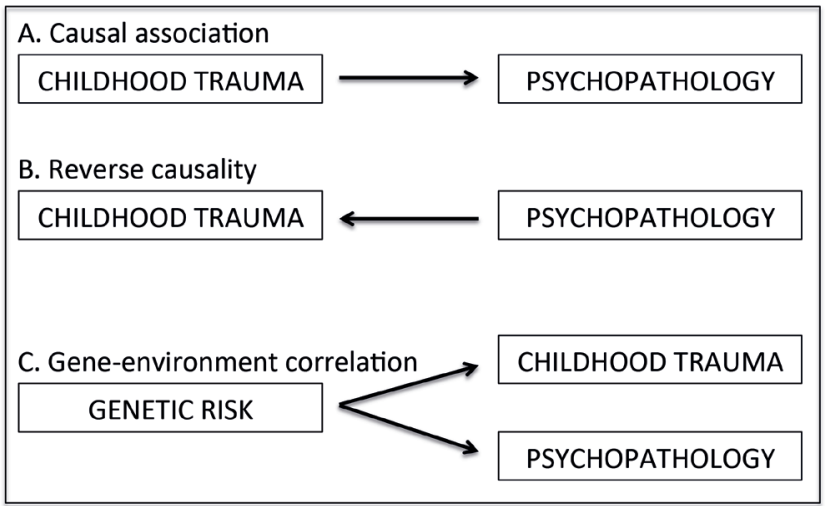

A. Exposure to childhood trauma induces psychopathology.

B. Vulnerability for psychopathology increases the likelihood of victimization.

C. Genetic risk directly increases the risk for psychopathology and also increases the risk for childhood trauma.

Especially in relation to psychosis, the question of a possible causal contribution is strongly debated. Nonetheless, some very promising results have been published, which do argue strongly for a causal association. For example, Kelleher and colleagues found, by extensively following a large sample of children for several years, that abusive events were associated with psychotic symptoms, but, more importantly, that psychotic symptoms attenuated after cessation of abusive events ${ }^{22}$. In plain terms: children displayed more (mild) psychotic symptoms when they were being bullied, but as time progressed, and the bullying stopped, the psychotic symptoms decreased or vanished completely ${ }^{22}$. Another important clue pointing towards a causal association is the consistently found 'dose-response' relationship between trauma and psychosis; as individuals report more trauma, they also display more severe psychotic symptoms ${ }^{23}$. 


\section{Psychological mechanisms}

Building on this body of research, i.e. the question whether the association is there, another important question that remains to be answered is how this association works. Moreover, finding a plausible underlying mechanism corroborates evidence of a causal association. Thus, there is a large body of work that tries to explain the trauma-psychosis link through psychological mechanisms. For example, it is thought that extreme childhood traumatic experiences can cause life-long post-traumatic reaction $\mathrm{s}^{24}$. A certain cue, like a sound or a smell, can cause a severe stress reaction in traumatized individuals. But for some individuals, no external cue is needed, and the sensory experience comes from within, thus hallucinations develop. Others postulate that being rejected by parents and being placed in foster care, possibly also growing up in an unsafe environment, will understandably induce overly distrusting ideas about others ("no one can be trusted"), which can lead to paranoid delusions throughout adulthood ${ }^{25}$. Then there are researchers that explain how certain psychotic symptoms can be compounded by others, influenced by traumatic events. For example, it has been found that traumatized individuals are more likely to develop delusional ideas about hallucinatory experiences ("the knocking on the door I hear is a ghost", instead of "I sometimes hear knocking when there's no one there"), than their non-traumatized counterparts ${ }^{26}$. This is thought to stem from a detrimental cognitive effort to make sense of hallucinatory experiences ${ }^{27}$, which is more likely to occur in traumatized individuals. Lastly, the 'social defeat' hypothesis of psychosis has been postulated, which is actually a combination of a psychological and a biological mechanism ${ }^{28}$. This hypothesis, built on animal research, entails that exposure to a certain risk factor, such as abusive events, leads to a decreased self-value in relation to others, which, through abnormalities in dopaminergic neurotransmission, can lead to psychotic symptoms ${ }^{28}$.

\section{Genetic underpinnings}

The idea that schizophrenia is mainly a biological disorder, and mostly independent of environmental influence, stems from convincing family-based studies. Up to $80 \%$ of the liability for psychotic disorder is thought to be due to genetic vulnerability ${ }^{29}$. The chances of developing schizophrenia when someone has an affected monozygotic twin are about $50 \%{ }^{29}$. However, many researchers argue that schizophrenia develops not solely based on genetic vulnerability, but from gene-environment interaction ${ }^{30}$. Moreover, based on many years of genetic research, researchers came to the conclusion that there is not one gene that causes schizophrenia, but many genes, all with a very small effect ${ }^{31}$. Now, state-ofthe-art methods for assessing effects of all of these genes together are being implemented, resulting in a so-called 'polygenic risk scores ${ }^{32}$. In essence, this score is obtained by calculating the overall genetic risk based on all genes found to be associated with schizophrenia in genome wide association studies (GWAS). Contrary to studies focused on particular SNPs, associations with psychosis in studies using polygenic risk scores are being replicated ${ }^{33}$. Possible interactions with childhood traumatic experiences are not yet explored. 
All studies discussed above have one aspect in common: each study has a top-down approach, i.e. they start from a certain set of symptoms (e.g. symptoms of depression), or a particular disorder (e.g. schizophrenia). From that starting point, researchers attempt to identify risk factors or underlying mechanisms. Recently, a more bottom-up approach is suggested, thus using a risk factor, such as childhood trauma, as a starting point ${ }^{34}$. One reason for this is the fact that within certain clinical groups, for example within a group of patients with schizophrenia, there is a large amount of heterogeneity in terms of disease severity and functional outcome. One important indicator for this heterogeneity is childhood trauma history: traumatized patients have a lower social or vocational functional outcome, increased disease severity, and lower quality of life than patients without a trauma history ${ }^{35}$. Furthermore, evidence has been reported that individuals exposed to trauma have higher rates of comorbidities; albeit in the form of multiple diagnoses ${ }^{36}$, or co-occurrence of symptoms ${ }^{26}$. Thus, stratifying according to trauma history can yield valuable progress in our understanding of mental illness.

\section{Aims of this thesis}

The aims of this thesis are a further investigation into the mechanisms that link childhood traumatic experiences to psychopathology. Given the relatively newer field of trauma research in association with psychosis, the first part (chapters two through seven) will have a deeper focus on this association, investigating possible underlying mechanisms. The second part (chapters eight through eleven) will broaden the scope, including other types of psychopathology such as depression and anxiety. Using this approach, the viability of stratifying according to childhood trauma history is investigated.

This led to the following research topics:

Chapter two delves in to the different methods used to establish psychotic experiences, comparing self-report measures to clinical interviews. In particular, the characteristics of the group of individuals who self-reported a psychotic experience, which were assessed as 'false positive' after a formal clinical interview (i.e. the clinician performing this latter interview decided that the self-reported psychotic experience was not, in fact, a psychotic experience) are investigated.

Chapter three investigates which factors (environmental or genetic) are associated with the transition to psychotic disorder, in a sample of non-affected siblings of patients with a psychotic disorder, and a sample of controls (individuals without a psychotic disorder, and without first or second degree relatives with a psychotic disorder).

In chapter four, several theories previously proposed by other researchers about psychological mechanisms that may explain the association between childhood trauma and psychosis are investigated. These theories include: (1) childhood trauma is associated with co-occurring hallucinations and delusions, rather then either symptom in isolation; 
(2) certain types of trauma have a stronger association with delusions, whereas other types are more strongly associated with hallucinations; (3) psychosis is more likely to develop in response to abusive events, rather than neglect; and (4) childhood trauma with an intentional nature (e.g. abuse) has a stronger association with psychosis than trauma without intent (e.g. accidents).

Chapter five reviews the available evidence of the association between trauma and psychosis, the nature of this association (causal or otherwise), and possible underlying biological and psychological mechanisms. The 'social defeat' hypothesis, as one example, is highlighted. Based on this discussion, combined with evidence found in chapter four, chapter six investigates the plausibility of the 'social defeat' hypothesis. In this study, the extent to which specific feelings, expressing a state of social defeat, mediate the link between childhood trauma and psychosis, is investigated.

Chapter seven investigates the possible causal nature of the association between childhood trauma and psychosis, by utilizing the newly available polygenic risk scores. Apart from attempting to replicate the association between polygenic risk and psychosis, this chapter investigated whether the association between trauma and psychosis is based on geneenvironment correlation.

In chapter eight the differential impact of trauma on several types of symptoms (positive psychotic, negative psychotic, and depressive symptoms) is investigated in a sample of patients with a psychotic disorder, their unaffected siblings, and controls. Additionally, the impact of trauma on the course of symptoms over a period of three years is assessed.

Chapter nine discusses the impact of presence of psychotic symptoms in individuals with an anxiety or mood disorder, in terms of disease severity and service use. Exposure to risk factors, such as drug use or childhood trauma, is assessed, comparing individuals with or without psychotic symptoms.

In chapter ten symptom profiles, including psychotic, anxious, mania, and depressive symptoms, of survivors of childhood trauma, are compared with symptom profiles of their non-traumatized counterparts. This chapter assesses whether traumatized individuals are more likely to develop any of these four symptom clusters in isolation, or rather combinations of these symptom clusters (i.e. clustering of symptoms).

Chapter eleven, based on findings in chapter ten, assesses the functional outcome of patients with an anxiety disorder, a mood disorder, or a psychotic disorder. Individuals exposed to trauma who also report clustering of symptoms are compared with individuals not exposed to trauma, without clustering of symptoms, or neither. 


\section{REFERENCES}

1. Bellis MA, Hughes $\mathrm{K}$, Leckenby $\mathrm{N}$, Perkins C, Lowey $\mathrm{H}$. National household survey of adverse childhood experiences and their relationship with resilience to health-harming behaviors in England. BMC medicine 2014;12:72.

2. Craig $W$, Harel-Fisch $Y$, Fogel-Grinvald $\mathrm{H}$, et al. A cross-national profile of bullying and victimization among adolescents in 40 countries. Int $j$ public health Sep 2009;54 Suppl 2:216-224.

3. May-Chahal C, Cawson P. Measuring child maltreatment in the United Kingdom: a study of the prevalence of child abuse and neglect. Child Abuse Negl Sep 2005;29(9):969-984.

4. Jespersen AF, Lalumiere ML, Seto MC. Sexual abuse history among adult sex offenders and non-sex offenders: a meta-analysis. Child Abuse Negl Mar 2009;33(3):179-192.

5. Bedard-Gilligan $\mathrm{M}$, Jaeger J, EchiverriCohen A, Zoellner LA. Individual differences in trauma disclosure. J beh ther exp psychiat Jun 2012;43(2):716-723.

6. Ullman SE. Relationship to perpetrator, disclosure, social reactions, and PTSD symptoms in child sexual abuse survivors. J child sexual $a b$ 2007;16(1):19-36.

7. De Graaf R, Ten Have M, van Gool C, Van Dorsselaer S. Prevalence of mental disorders and trends from 1996 to 2009. Results from the Netherlands Mental Health Survey and Incidence Study-2. Soc Psychiat and Psychiatr Epidem 2012;47(2):203-213.
8. van Os J, Hanssen M, Bijl RV, Vollebergh W. Prevalence of psychotic disorder and community level of psychotic symptoms. Arch Gen Psychiat 2001;58:663-668.

9. de Graaf $R$, ten Have M, van Dorsselaer S. De psychische gezondheid van de Nederlandse bevolking. Utrecht: Trimbos-instituut; 2010.

10. Linscott RJ, Van Os J. Systematic reviews of categorical versus continuum models in psychosis: evidence for discontinuous subpopulations underlying a psychometric continuum. Implications for DSM-V, DSM-VI, and DSM-VII. Ann Rev Clin Psychol 2010;6:391-419.

11. Kelleher I, Harley M, Murtagh A, Cannon M. Are screening instruments valid for psychotic-like experiences? A validation study of screening questions for psychotic-like experiences using in-depth clinical interview. Schizophr Bull 2009;37(2):362-369.

12. Buckley PF, Miller BJ, Lehrer DS, Castle DJ. Psychiatric comorbidities and schizophrenia. Schizophr Bull Mar 2009;35(2):383-402.

13. Fusar-Poli $P$, Nelson $B$, Valmaggia $L$, Yung AR, McGuire PK. Comorbid Depressive and Anxiety Disorders in 509 Individuals With an At-Risk Mental State: Impact on Psychopathology and Transition to Psychosis. Schizophr Bull 2012;doi:10.1093/schbul/sbs136. 
14. Perrin $M$, Vandeleur $C L$, Castelao $E$, Rothen S, Glaus J, Vollenweider P, Preisig M. Determinants of the development of post-traumatic stress disorder, in the general population. Soc Psychiatry Psychiatr Epidem Mar 2014;49(3):447-457.

15. Nanni V, Uher R, Danese A. Childhood maltreatment predicts unfavorable course of illness and treatment outcome in depression: a meta-analysis. Am J Psychiat 2012;169:141-151.

16. Kuo JR, Goldin PR, Werner K, Heimberg RG, Gross JJ. Childhood trauma and current psychological functioning in adults with social anxiety disorder.

J Anx Disord 2011;25(4):467-473.

17. Varese F, Smeets F, Drukker M, et al. Childhood adversities increase the risk of psychosis:

a meta-analysis of patient-control, prospective- and cross-sectional cohort studies. Schizophr Bull 2012;38(4):661-671.

18. Read J, Bentall R, Fosse R. Time to abandon the bio-bio-bio model of psychosis: exploring the epigenetics and psychological mechanisms by which adverse life events lead to psychotic symptoms. Epidem Psichiatr Soc 2009;18(4):299-310.

19. Fisher HL, Craig TK, Fearon $P$, et al. Reliability and comparability of psychosis patients' retrospective reports of childhood abuse. Schizophr Bull 2011;37(3):546-553.

20. Sideli L, Mule A, La Barbera D, Murray RM. Do child abuse and maltreatment increase risk of schizophrenia?

Psychiat invest Jun 2012;9(2):87-99.
21. Fisher H, McGuffin P, Boydell J, et al. Interplay between childhood physical abuse and familial risk in the onset of psychotic disorders. Schizophr Bull 2014;doi: 10.1093/schbul/sbt201.

22. Kelleher I, Keeley H, Corcoran P, et al. Childhood trauma and psychosis in a prospective cohort study: cause, effect, and directionality. Am J Psychiatry 2013;170(7):734-741.

23. Heins $M$, Simons $C$, Lataster $T$, et al. Childhood trauma and psychosis: $A$ case-control and case-sibling comparison across different levels of genetic liability, psychopathology, and type of trauma. Am J Psychiatry 2011;168(12):1286-1294.

24. Read J, Agar K, Argyle N, Aderhold V. Sexual and physical abuse during childhood and adulthood as predictors of hallucinations, delusions and thought disorder. Psychol Psychother: Theor Res Pract 2003;76:1-22.

25. Bentall R, Wickham S, Shevlin M, Varese F. Do specific early-life adversities lead to specific symptoms of psychosis? A study from the 2007 the adult psychiatric morbidity survey. Schizophr Bull 2012;38(4):734-740.

26. Smeets F, Lataster T, Dominguez MG, Hommes J, Lieb R, Wittchen HU, van Os J. Evidence That Onset of Psychosis in the Population Reflects Early Hallucinatory Experiences That Through Environmental Risks and Affective Dysregulation Become Complicated by Delusions. Schizophr Bull 2012;38(3):531-542. 
27. Kapur S. Psychosis as a state of aberrant salience: a framework linking biology, phenomenology, and pharmacology in schizophrenia. Am J Psychiatry 2003;160:13-23.

28. Selten JP, Cantor-Graae E. Hypothesis: social defeat is a risk factor for schizophrenia? B J Psychiatry 2007;191(suppl. 51):s9-s12.

29. Sullivan PF, Kendler KS, Neale MC. Schizophrenia as a complex trait: evidence from a meta-analysis of twin studies. Arch Gen Psychiatry Dec 2003;60(12):1187-1192.

30. van Os J, Kenis G, Rutten BPF. The environment and schizophrenia. Nature 2010;468(7321):203-212.

31. Singh $S$, Kumar $A$, Agarwal $S$, Phadke SR, Jaiswal Y. Genetic insight of schizophrenia: past and future perspectives. Gene Feb 10 2014;535(2):97-100.

32. Purcell SM, Wray NR, Stone JL, Visscher PM, O'Donovan MC, Sullivan PF, Sklar P. Common polygenic variation contributes to risk of schizophrenia and bipolar disorder. Nature 2009;460(7256):748-752.
33. Power RA, Verweij KJ, Zuhair M, et al. Genetic predisposition to schizophrenia associated with increased use of cannabis. Mol Psychiatry Jun 242014.

34. Teicher MH, Samson JA. Childhood maltreatment and psychopathology: A case for ecophenotypic variants as clinically and neurobiologically distinct subtypes. Am J Psychiatry Oct 1 2013;170(10):1114-1133.

35. Boyette LL, van Dam D, Meijer C, et al. Personality Compensates for Impaired Quality of Life and Social Functioning in Patients With Psychotic Disorders Who Experienced Traumatic Events. Schizophr Bull Apr 252014.

36. Banducci AN, Hoffman E, Lejuez CW, Koenen KC. The relationship between child abuse and negative outcomes among substance users: psychopathology, health, and comorbidities. Addict Behav Oct 2014;39(10):1522-1527. 
20 | Surviving Childhood 


\title{
CHAPTER 2
}

\section{Phenotypically Continuous with Clinical Psychosis, Discontinuous in Need for Care: Evidence for an Extended Psychosis Phenotype}

\author{
Martine van Nierop ${ }^{A}$ \\ Jim van $O s^{A B}$ \\ Nicole Gunther ${ }^{A C}$ \\ Inez Myin-Germeys ${ }^{A}$ \\ Ron de Graaf ${ }^{\mathrm{D}}$ \\ Margreet ten Have \\ Saskia van Dorsselaer ${ }^{\mathrm{D}}$ \\ Maarten Bak ${ }^{\mathrm{A}}$ \\ Ruud van Winkel ${ }^{\mathrm{AE}}$
}

\footnotetext{
A Dept. of Psychiatry and Psychology, School for Mental Health and Neuroscience, EURON, Maastricht University Medical Centre, PO Box 616 (Vijv1), 6200 MD Maastricht, The Netherlands

${ }^{B}$ King's College London, King's Health Partners, Department of Psychosis Studies, Institute of Psychiatry, London, UK

c School of Psychology, Open University, The Netherlands

D Netherlands Institute of Mental Health and Addiction, Da Costakade 45, 3521 VS Utrecht, The Netherlands

E University Psychiatric Center, Katholieke Universiteit Leuven, Campus Kortenberg, Leuvensesteenweg, Kortenberg, Belgium
} 


\section{ABSTRACT}

\section{Background}

Rates of self-reported psychotic experiences (SRPEs) in general population samples are high; however the reliability against interview-based assessments and the clinical significance of false-positive (FP) ratings remain unclear.

\section{Design}

The second Netherlands Mental Health Survey and Incidence Study, a general population study.

\section{Methods}

Trained lay interviewers administered a structured interview assessing psychopathology and psychosocial characteristics in 6646 participants. Participants with at least one SRPE $(\mathrm{N}=1084)$ were reassessed by clinical telephone interview.

\section{Results}

Thirty-six percent of participants with SRPEs were confirmed by clinical interview as true positive (TP). SPREs not confirmed by clinical interview (FP group) generated less helpseeking behavior and occurred less frequently compared with TP experiences (TP group). However, compared with controls without psychotic experiences, the FP group more often displayed mood disorder (relative risk [RR] 1.7, 1.4 - 2.2), substance use disorder (RR 2.0, 1.6 - 2.6), cannabis use (RR 1.5, 1.2-1.9), higher levels of neuroticism (RR 1.8, 1.5 2.2 ), affective dysregulation, and social dysfunction. The FP group also experienced more sexual (RR 2.0, 1.5 - 2.8) and psychological childhood trauma (RR 2.1, 1.7-2.6) as well as peer victimization (RR 1.5, 1.2 - 2.0), and recent life events (RR 2.0, 1.6-2.4) than controls without psychotic experiences. Differences between the FP group and the TP group across these domains were much smaller and less conclusive.

\section{Discussion}

SRPEs not confirmed by clinical interview may represent the softest expression of an extended psychosis phenotype that is phenotypically continuous with clinical psychosis, but discontinuous in need for care. 


\section{INTRODUCTION}

Extended phenotypes of psychotic disorder in the general population are thought to reflect the behavioral expression of distributed population risk ${ }^{1}$. Although psychometric expressions of risk in the general population are transitory in the majority of individuals, progression to clinical outcome may occur depending on the rate of persistence ${ }^{2}$; degree of "comorbid" admixture of dimensions of negative symptoms, affective dysregulation and reality distortion ${ }^{3,4}$; level of coping; number, frequency, severity and associated distress of psychotic experiences ${ }^{6-8}$; and level of premorbid social functioning ${ }^{6}$. Poulton and colleagues demonstrated that an assessment of "definite" psychotic experience carried a higher likelihood of transition to clinical psychotic disorder over a 16-year followup period than a rating of "likely" psychotic experience". These data suggest that accurate assessment of psychotic experiences is important in relation to the degree of associated risk. A systematic review of 285 rates of prevalence or incidence of psychotic experiences showed that half of the considerable heterogeneity in rates of subclinical psychotic experiences across studies is due to study cohort and design factors ${ }^{8}$. Particularly, rates were found to be much higher in studies using smaller $n$, convenience sampling, and selfreport assessment.

Self-reports of psychotic experiences generate "false-positive" (FP) ratings. Depending on how data are analyzed, the rate of FP self-reported psychotic experiences (SRPEs) when verified by clinical interview may vary from $7 \%^{9}$ to $61 \%^{10}$. There is evidence, however, that $\mathrm{FP}$ in this context does not indicate absence of risk. Thus, Bak and colleagues found that FP psychotic experiences (i.e. the presence of Composite International Diagnostic Interview [CIDI] SRPEs that were not confirmed by clinical interview) nevertheless were strongly associated with future psychotic disorder, albeit at a lower level than confirmed psychotic experiences ${ }^{11}$.These findings echo those by Poulton and colleagues regarding definite and likely psychotic symptoms, and suggest that SRPEs do not come as either 'true positive' (TP) or FP. Instead, they may index risk as a continuum reflecting the level of certainty as to what degree the experience that an individual reports can be regarded as "psychotic" factors related to the expression of mild psychotic experiences and how they eventually lead to need for care.

In the present study, the relationship between SRPES and assessment of psychosis by clinical interview was therefore investigated. First, positive predictive values (PPVs) of several different psychotic experiences were established. Low PPVs were hypothesized, with higher PPVs for hallucinations than for delusions ${ }^{10}$. In line with Bak and colleagues ${ }^{11}$, it was further hypothesized that individuals with FP psychotic experiences would be more similar to the group with confirmed psychotic symptoms than to the control group in terms of psychopathology and exposure to environmental risk factors associated with psychotic disorder ${ }^{12}$. 


\section{METHODS}

This study forms part of the recent second Netherlands Mental Health Survey and Incidence Study (NEMESIS-2), an entirely new longitudinal study of the prevalence, incidence, course, and consequences of psychiatric disorders in the Dutch general population. NEMESIS- 2 replicates and extends the first NEMESIS-1 study, conducted from 1996 to $1999^{13}$, in an independent, nonoverlapping sample. The study was approved by the Medical Ethics Review Committee for Institutions on Mental Health Care. For a more detailed description of the NEMESIS-2 method see De Graaf et al. ${ }^{14,15}$

\section{Instruments - First Interview}

The participants were interviewed at home by trained interviewers who are not clinicians with the CIDI version 3.0 ${ }^{16,17}$. Demographics, somatic health, life events, treatmentseeking, and different vulnerability factors were also assessed, such as neuroticism, using the Eysenck Personality Questionnaire (the EPQ-revised short scale) ${ }^{18,19}$ and childhood trauma (emotional, physical, psychological and sexual abuse, and peer victimization), using self-constructed questionnaires. Cannabis use was assessed in the section Illegal Substance Use of the CIDI 3.0, and analyzed, conform previous analyses in NEMESIS-120, as two dichotomous variables indicating lifetime use and regular use. Continuous ratings of general mental and physical health and social functioning were assessed by the Medical Outcomes Study Short-form Health Survey (SF-36) ${ }^{21,22}$. For a full overview of the assessment instruments in addition to the CIDI 3.0, see De Graaf et al. ${ }^{14}$

Studies on earlier CIDI versions concluded that the CIDI assesses disorders with generally acceptable reliability and validity, with the exception of psychosis ${ }^{23,24}$. As CIDI methodology to assess psychotic experiences in versions of CIDI 1 and CIDI 2 was not included in CIDI 3.0, a psychosis add-on instrument was constructed, based on the section of psychotic symptoms in CIDI versions 1 and 2. This part of the interview consisted of 20 psychotic experiences, each rated 'yes', 'no', 'don't know' or 'refuse', over the lifetime period. Whenever a psychotic experience was endorsed, the subject was asked to state, on a 1 (rarely) to 4 (almost always) scale, how often this experience occurred (Frequency), how much it bothered them (Distress), and to what extent the experience had an influence on their daily professional and social activities (Impact). The sum scores for frequency and impact of psychotic experiences, as well as distress by psychotic experiences were calculated as the mean of the sum scores of these items across the 20 psychotic experiences. Psychotic experiences were considered secondary if all endorsed psychotic items were caused by use of drugs/alcohol or physical illness. Because clinical relevance of psychotic experiences may be difficult to diagnose by lay interviewers ${ }^{25,26}$, and the interviewers made no clinical judgment about participants' answers, the reported experiences may be considered an extension of 'self-report'. 
Consistent with work in NEMESIS-1 ${ }^{27}$ and other CIDI-based population work ${ }^{4}$, a lifetime depression score was obtained by adding up 28 symptom items (present/not present) from the CIDI 3.0 Depression section. Lifetime and past-year mania scores were calculated similarly ${ }^{4,27}$.

Lastly, participants were asked about help seeking in the context of any psychopathology (help from psychiatrists/psychologists for any psychiatric problem including drug or alcohol problems) and help seeking specifically for psychotic experiences.

\section{Instruments - reinterview}

Individuals who endorsed at least one lifetime psychotic experience (1078 out of 6646 participants) were contacted for reinterview over the telephone by an experienced clinician at the level of psychologist or psychiatrist within eight weeks after the initial interview, as in NEMESIS- ${ }^{11}$. Reinterviews were conducted using questions from the Structured Clinical Interview for DSM-IV (SCID-I), an instrument with proven reliability and validity in diagnosing psychotic disorders ${ }^{28}$. Findings from all reinterviews were discussed with a second clinician (M.B.), who also conducted and supervised the clinical reinterviews in NEMESIS-1.

\section{Sample and Prevalences}

The total sample consisted of 6646 participants (response rate 65\%; see table 1 for characteristics). As this was a study of relative rather than absolute risk, data were not weighted, and therefore proportions may be different from weighted estimates of absolute risk presented elsewhere ${ }^{14,15}$.

The sample that participated in the reinterview consisted of 792 participants (participation rate: $74 \%$, see table 1 ). No significant differences existed between those that participated in the clinical reinterview and those that did not with regard to age, lifetime Axis I disorders, gender, educational level, or employment status. However, nonparticipants more often had self-reports of psychotic experiences (mean $=2.5$ [95\% Cl: 2.3-2.8]) compared with participants $($ mean $=1.9[95 \% \mathrm{Cl}: 1.8-2.0])$. 
Table 1 NEMESIS-2 Baseline Sample and Nonweighted Prevalences - First Interview and Reinterview

\begin{tabular}{|c|c|c|c|c|}
\hline & First interview, $\mathbf{N}$ & Prevalence (\%) & Re-interview, $\mathrm{N}$ & Prevalence (\%) \\
\hline Total sample & 6646 & & 792 & \\
\hline \multirow[t]{2}{*}{ Men } & 2974 & 45 & 300 & 38 \\
\hline & & Mean age $44.4 y$ (SD 12.6) & & Mean age 42.8y (SD 13.0) \\
\hline \multirow[t]{2}{*}{ Women } & 3667 & 55 & 492 & 62 \\
\hline & & Mean age 44.1y (SD 12.5) & & Mean age 44.2y (SD 12.4) \\
\hline Paid job & 4952 & 75 & 570 & 72 \\
\hline No paid job & 1689 & 25 & 222 & 28 \\
\hline \multicolumn{5}{|l|}{ Education } \\
\hline Primary education & 332 & 5 & 47 & 6 \\
\hline Lower secondary education & 1825 & 28 & 234 & 30 \\
\hline Higher secondary education & 2145 & 32 & 281 & 36 \\
\hline $\begin{array}{l}\text { Higher professional/ } \\
\text { university education }\end{array}$ & 2339 & 35 & 230 & 29 \\
\hline $\begin{array}{l}\text { Self-reported psychotic } \\
\text { experience }\end{array}$ & 1078 & 16 & 384 & 6 \\
\hline Lifetime mood disorder ${ }^{b}$ & 1389 & 21 & 270 & 34 \\
\hline Lifetime anxiety disorder $^{c}$ & 1333 & 20 & 252 & 32 \\
\hline Lifetime substance use disorder ${ }^{d}$ & 1127 & 17 & 213 & 27 \\
\hline
\end{tabular}

Note: primary education $=$ no education or (part of) primary school. ICD, International Classification of Diseases.

${ }^{a}$ Of a list of 20 positive psychotic experiences.

${ }^{\mathrm{b}}$ Any DSM-IV or ICD-10 lifetime mood disorder diagnosis, generated by CIDI 3.0.

'Any DSM-IV or ICD-10 lifetime anxiety disorder diagnosis, generated by CIDI 3.0.

${ }^{\mathrm{d} A n y}$ DSM-IV or ICD-10 lifetime substance disorder diagnosis, generated by CIDI 3.0.

\section{Analyses}

PPVs of SRPEs were calculated with clinical ratings as gold standard, using STATA, version $10^{29}$. Participants were divided into three groups, based on their symptom profiles: (1) participants who did not report any psychotic experience (control group); (2) participants who reported one or more psychotic experiences, none of which were confirmed at clinical interview (false positive [FP] group); (3) participants who reported one or more psychotic experiences and for whom at least one psychotic experience was confirmed during clinical interview (true positive [TP] group). Multinomial logistic regression was applied using group as dependent variable, a priori controlling for age and gender. Associations with categorical predictors were expressed as Relative Risk Ratio (RR), while associations with continuous variables were expressed as B-coefficient, comparing the FP group and the TP group with the controls (the reference group), and comparing the TP group with the FP group (using FP as reference). 


\section{RESULTS}

\section{Psychotic experiences}

The prevalence of at least one SRPE over the lifetime was 16\% (1078 out of 6646), similar to the lifetime rate of $18 \%$ in NEMESIS- $1^{30}$. The prevalence of at least one lifetime true psychotic experience (confirmed by the clinician) was $6 \%$ (384 out of 6360), which could not be compared with NEMESIS-1 due to methodological differences ${ }^{30}$. The PPVs for delusions were generally lower (20\%-50\%) than for hallucinations (45\%-60\%; table 2 ). The prevalence of psychotic disorder was $0.7 \%$ (43 out of 6646). Of the 43 participants with a diagnosis of psychotic disorder, 22 had been available for clinical reinterview with the SCID; the remaining 21 participants received the diagnosis of psychotic disorder based on CIDI interview data only.

Table 2 Positive Predictive Values for Self-reported Psychotic Experiences in Baseline Assessment of the NEMESIS-2 Sample, Compared with Clinical Interview

\begin{tabular}{|c|c|c|c|}
\hline Type of Psychotic Experience & PPV $(95 \% \mathrm{Cl})$ & N Lay Interview (\%) & $\begin{array}{l}\text { N Clinical } \\
\text { Interview (\%) }\end{array}$ \\
\hline \multicolumn{4}{|l|}{ Delusions } \\
\hline Being spied on & $33.5(27.8-39.1)$ & $379(6)$ & $91(1)$ \\
\hline Being followed & $27.2(20.2-34.3)$ & $228(3)$ & $44(1)$ \\
\hline Being subject of secret testing & $23.8(11.1-36.5)$ & $70(1)$ & $11(0.2)$ \\
\hline Conspiracy & $34.3(23.6-45.0)$ & $102(2)$ & $30(1)$ \\
\hline Familiar person been replaced by 'double' & n.e.d. & $4(0.1)$ & 0 \\
\hline Thoughts being read by others & $26.6(16.0-37.1)$ & $93(1)$ & $20(0.3)$ \\
\hline Hearing someone's thoughts & $18.0(9.5-26.4)$ & $115(2)$ & $16(0.2)$ \\
\hline Thought broadcasting & $27.1(14.6-39.5)$ & $80(1)$ & $13(0.2)$ \\
\hline Thought insertion & $37.9(20.9-55.0)$ & $55(1)$ & $13(0.2)$ \\
\hline Thoughts taken away & n.e.d. & $18(0.3)$ & $4(0.1)$ \\
\hline Special messages through radio or television & $47.6(27.2-68.0)$ & $33(1)$ & $12(0.2)$ \\
\hline Hypnotized or charmed by strange forces & $34.6(17.0-52.2)$ & $41(1)$ & $11(0.2)$ \\
\hline Thoughts or actions controlled & $43.5(24.1-62.9)$ & $45(1)$ & $13(0.2)$ \\
\hline Thoughts influenced by appliances & $30.0(2.9-57.1)$ & $17(0.3)$ & $4(0.1)$ \\
\hline Other delusions & $48.5(41.0-56.1)$ & $94(1)$ & $131(2)$ \\
\hline \multicolumn{4}{|l|}{ Hallucinations } \\
\hline Visual & $49.5(43.0-56.1)$ & $305(5)$ & $114(2)$ \\
\hline Auditory & $58.6(49.3-68.0)$ & $138(2)$ & $69(1)$ \\
\hline Thought echo & n.e.d. & $49(1)$ & $8(0.1)$ \\
\hline Olfactory & $59.8(49.5-70.0)$ & $136(2)$ & $54(1)$ \\
\hline Tactile & $47.8(40.1-55.4)$ & $225(3)$ & $81(1)$ \\
\hline
\end{tabular}

Note: n.e.d., not enough data for this type of experience; PPV, Positive Predictive Value 


\section{Group comparisons}

The prevalence rates and means of the various psychopathological and psychosocial variables are shown in tables 3 and 4. The FP group, similar to the TP group, had significantly higher RRs than the control group for a lifetime mood disorder, a lifetime substance use disorder, as well as for level of neuroticism, regular cannabis use and childhood sexual trauma (table 5). In the comparison between TP and FP, no significant differences were found for exposure to childhood sexual trauma, negative life events in the past year, lifetime cannabis use, and whether psychotic experiences were secondary to drug or alcohol use or a somatic condition. Even for variables that were significantly different in the comparison between FP and TP groups, RRs were smaller than for the corresponding comparisons between the FP group and controls (table 5). The TP group more often reported help seeking behavior for psychotic experiences (RR 3.79 [2.27-6.34]) and also more often sought help in the context of any psychopathology than the FP group (RR 1.86 [1.22-2.83]).

Table 3 NEMESIS-2 Baseline Assessment - Prevalence Rates for Dichotomous Variables, Psychopathology, and Environmental Risk Factors

\begin{tabular}{|c|c|c|c|}
\hline Dichotomous Variable & Controls N (\%) & FP N (\%) & TP N (\%) \\
\hline Lifetime mood disorder & $989 / 5453(18)$ & $115 / 408(28)$ & $155 / 384(40)$ \\
\hline Lifetime anxiety disorder & $951 / 5453(17)$ & $105 / 408(26)$ & $147 / 384(38)$ \\
\hline Lifetime substance dependence/abuse disorder & $807 / 5453(15)$ & $96 / 408(24)$ & $117 / 384(31)$ \\
\hline Neuroticism (dichotomous: high/low) & $1725 / 5332(32)$ & $191 / 404(47)$ & $220 / 379(58)$ \\
\hline Sexual abuse $<16$ & $351 / 5333(7)$ & $53 / 404(13)$ & $68 / 379(18)$ \\
\hline Physical abuse $<16$ & $357 / 5333(7)$ & $48 / 404(12)$ & $74 / 379(20)$ \\
\hline Emotional abuse $<16$ & $685 / 5333(13)$ & $99 / 404(25)$ & $135 / 379(36)$ \\
\hline Psychological abuse $<16$ & $773 / 5333(15)$ & $105 / 404(26)$ & $128 / 379(34)$ \\
\hline Regular peer victimization $<16$ & $675 / 5329(13)$ & $73 / 404(18)$ & $104 / 379(27)$ \\
\hline Negative life events past year & $2541 / 5333(48)$ & $259 / 404(64)$ & $249 / 379(66)$ \\
\hline Cannabis use lifetime (at least once) & $1148 / 5452(21)$ & $110 / 408(27)$ & $117 / 384(31)$ \\
\hline Regular cannabis use (at least once/wk) & $253 / 5329(5)$ & $27 / 394(7)$ & $43 / 366(12)$ \\
\hline Help seeking, general ${ }^{a}$ & $273 / 5333(5)$ & $40 / 404(10)$ & $65 / 379(17)$ \\
\hline Help seeking, specific ${ }^{b}$ & na & $21 / 407(5)$ & $66 / 384(17)$ \\
\hline Secondary experience ${ }^{c}$ & na & $10 / 406(3)$ & $17 / 381(5)$ \\
\hline
\end{tabular}

Note: na, not applicable; FP, false-positive group; TP, true positive group.

aGeneral: psychiatric problems, including drug- or alcohol-related help seeking.

bspecific: psychotic experiences.

'Psychotic experiences secondary to drug or alcohol use or somatic condition. 
Table 4 NEMESIS-2 Baseline Assessment - Means, Minimum, Maximum and SDs for Continuous Variables; Psychopathology, General Health and Social Functioning, and Severity of Psychotic Experiences

\begin{tabular}{|c|c|c|c|}
\hline Continuous variable & $\begin{array}{l}\text { Controls mean } \\
\text { (min - max) (SD) }\end{array}$ & $\begin{array}{l}\text { FP mean } \\
(\min -\max )(S D)\end{array}$ & $\begin{array}{l}\text { TP mean } \\
(\min -\max )(S D)\end{array}$ \\
\hline Lifetime depression scale & $3.42(0-28)(6.77)$ & $5.05(0-26)(7.86)$ & $8.48(0-28)(9.68)$ \\
\hline Lifetime mania scale & $0.47(0-14)(1.42)$ & $1.07(0-15)(2.35)$ & $1.77(0-17)(3.20)$ \\
\hline Past year mania scale & $0.07(0-36)(1.11)$ & $0.34(0-18)(2.21)$ & $0.39(0-24)(2.47)$ \\
\hline General mental health & $84.56(8-100)(12.41)$ & $80.33(16-100)(15.23)$ & $78.41(12-100)(15.72)$ \\
\hline General physical health & $73.34(0-100)(17.46)$ & $67.82(0-100)(19.89)$ & $64.79(0-100)(20.25)$ \\
\hline General social functioning & $91.76(0-100)(16.68)$ & $87.04(0-100)(20.87)$ & $84.95(0-100)(22.95)$ \\
\hline Frequency psychotic experiences & na & $1.65(0-4)(0.75)$ & $1.85(.75-4)(0.76)$ \\
\hline Distress psychotic experiences & na & $1.78(0-4)(0.97)$ & $1.81(1-4)(0.92)$ \\
\hline Impact of psychotic experiences & na & $1.37(0.5-4)(0.72)$ & $1.41(1-4)(0.72)$ \\
\hline $\begin{array}{l}\text { Onset of psychotic experiences } \\
\text { (age in years) }\end{array}$ & na & $27.97(2-62)(13.12)$ & $25.46(1-62)(14.44)$ \\
\hline $\begin{array}{l}\text { Recency of psychotic } \\
\text { experiences }^{a}\end{array}$ & na & $1.64(1-6)(1.44)$ & $2.48(1-6)(1.97)$ \\
\hline
\end{tabular}

Note: min, minimum; max, maximum; na, not applicable; FP, false-positive group; TP, true positive group; SD, Standard Deviation ${ }^{a}$ Higher score for recency is more recent.

Compared with the control group, both the FP and the TP groups displayed higher levels of psychopathology, including lifetime depression and lifetime mania (table 5). In addition, they had worse physical health and social functioning (table 5). No significant FP-TP between-group differences were found for manic symptoms in the past year, general mental health, general social functioning, and impact of or distress by the psychotic experiences. The TP group displayed poorer general physical health and higher lifetime depression and mania scores compared with the FP group (table 5). The TP group scored higher on the frequency scale (B FP vs TP: 0.36 [0.17-0.55], mean $_{\mathrm{FP}}: 1.65, \mathrm{SD}_{\mathrm{FP}}: 0.75$, mean $\left._{\mathrm{TP}}: 1.85, \mathrm{SD}_{\mathrm{TP}}: 0.76\right)$ compared with the FP group. There was no significant difference in age at onset (mean: 26.72, SD: 13.83), but the TP group had more recent experience of psychosis than the FP group (B FP vs TP: 0.28 [0.19-0.37], mean $_{\mathrm{FP}}: 1.64, \mathrm{SD}_{\mathrm{FP}}: 1.44$, mean $\left._{\mathrm{TP}}: 2.48, \mathrm{SD}_{\mathrm{TP}}: 1.97\right)$. No participants in the FP group met criteria for psychotic disorder, whereas 22 participants in the TP group (6\%) did. 
Table 5 Comparisons of Control Group, FP Group and TP Group in Terms of Psychopathology and Environmental Risk Factors - Relative Risk Ratios and B Coefficients

\begin{tabular}{|c|c|c|c|}
\hline & \multicolumn{3}{|l|}{ Group Comparison } \\
\hline & $\begin{array}{l}\text { Controls (Reference) } \\
\text { vs FP RR ( } 95 \% \mathrm{Cl})\end{array}$ & $\begin{array}{l}\text { Controls (Reference) } \\
\text { vs TP RR ( } 95 \% \mathrm{Cl})\end{array}$ & $\begin{array}{l}\text { FP (Reference) vs TP } \\
\text { RR ( } 95 \% \mathrm{CI})\end{array}$ \\
\hline \multicolumn{4}{|l|}{ Dichotomous Variable (N) } \\
\hline Lifetime mood disorder (1259) & $1.72 * * *(1.37-2.16)$ & $2.96 * * *(2.38-3.68)$ & $1.72 * * *(1.27-2.32)$ \\
\hline Lifetime anxiety disorder (1203) & $1.59 * * *(1.26-2.01)$ & $2.85 * * *(2.29-3.54)$ & $1.79 * * *(1.32-2.42)$ \\
\hline Lifetime substance use disorder (1020) & $2.02 * * *(1.57-2.59)$ & $3.08 * * *(2.42-3.92)$ & $1.53 *(1.10-2.12)$ \\
\hline Neuroticism (2136 - dichotomous: high/low) & $1.82^{* * *}(1.48-2.24)$ & $2.77^{* * *}(2.24-3.44)$ & $1.52 * *(1.14-2.02)$ \\
\hline Sexual abuse <16 (472) & $2.04 * * *(1.49-2.79)$ & $2.90 * * *(2.16-3.88)$ & ns \\
\hline Physical abuse <16 (479) & $1.88^{* * *}(1.37-2.59)$ & $3.47^{* * *}(2.63-4.58)$ & $1.84^{* *}(1.24-2.73)$ \\
\hline Emotional abuse $<16$ (919) & $2.16^{* * *}(1.69-2.75)$ & $3.73 * * *(2.98-4.69)$ & $1.73 * *(1.26-2.36)$ \\
\hline Psychological abuse <16 (1006) & $2.09 * * *(1.65-2.64)$ & $3.04 * * *(2.42-3.81)$ & $1.46 *(1.07-1.98)$ \\
\hline Regular peer victimization <16 (852) & $1.50 * *(1.15-1.97)$ & $2.49 * * *(1.95-3.17)$ & $1.65^{* *}(1.18-2.33)$ \\
\hline Negative life events past year (3049) & $1.95^{* * *}(1.58-2.40)$ & $2.07 * * *(1.66-2.57)$ & ns \\
\hline Cannabis use lifetime (at least once) (1375) & $1.48^{* *}(1.17-1.89)$ & $1.72 * * *(1.36-2.19)$ & ns \\
\hline Regular cannabis use (at least once/wk) (323) & $1.62 *(1.07-2.47)$ & $3.01 * * *(2.11-4.30)$ & $1.85 *(1.10-3.12)$ \\
\hline Help seeking, general ${ }^{\mathrm{a}}$ & $2.02 * * *(1.43-2.87)$ & $3.76^{* * *}(2.80-5.05)$ & $1.86 * *(1.22-2.83)$ \\
\hline Continuous variable & $\mathrm{B}(95 \% \mathrm{Cl})$ & $\mathrm{B}(95 \% \mathrm{Cl})$ & $\mathrm{B}(95 \% \mathrm{Cl})$ \\
\hline Lifetime depression scale ${ }^{b}$ & $0.03^{* * *}(0.02-0.04)$ & $0.07^{* * *}(0.06-0.09)$ & $0.05^{* * *}(0.03-0.06)$ \\
\hline Lifetime mania scale ${ }^{c}$ & $0.17^{* * *}(0.12-0.21)$ & $0.25 * * *(0.21-0.28)$ & $0.08^{* *}(0.03-0.13)$ \\
\hline Past year mania scale ${ }^{c}$ & $0.10^{* * *}(0.05-0.15)$ & $0.10^{* * *}(0.06-0.15)$ & ns \\
\hline General mental health & $-0.02 * * *(-0.03--.02)$ & $-0.03 * * *(-0.04--.02)$ & ns \\
\hline General physical health & $-0.02 * * *(-0.02--.01)$ & $-0.03 * * *(-0.03--.02)$ & $-0.01 *(-0.02--0.00)$ \\
\hline General social functioning & $-0.01 * * *(-0.02--.01)$ & $-0.02 * * *(-0.02--.01)$ & ns \\
\hline
\end{tabular}

Note: ns, not significant; FP, false-positive group; TP, true positive group; RR, relative risk.

${ }^{a}$ General: psychiatric problems, including drug- or alcohol-related help seeking.

${ }^{\mathrm{b}}$ Expressed in number of depressive symptoms.

'Expressed in number of mania symptoms.

$* \mathrm{p}<.05, * * \mathrm{p}<.01, * * * \mathrm{p}<.001$. 


\section{DISCUSSION}

The current study found a high proportion of formally FP psychotic experiences in a large general population sample, comparing self-report with clinical interview. The present study also confirms earlier work $^{11}$ that FP psychotic experiences have clinical and prognostic relevance. Compared with the control group, the FP group was more likely to have mood, anxiety or substance use disorders, as well as higher levels of neuroticism. They also had higher rates of childhood trauma and peer victimization, were more likely to have experienced a negative life event in the past year and to have ever used cannabis. They had worse physical and mental health, worse social functioning and more symptomatic expression of depression and mania. Compared with those with confirmed psychotic experiences, however, associations with psychopathology, social functioning, environmental risk factors, and help seeking were generally weaker.

To the best of our knowledge, this is the first study to investigate the characteristics of individuals with FP psychotic experiences. It confirms the findings of earlier studies that self-report questionnaires for psychotic experiences yield high rates of formally FPs ${ }^{9}, 10,31$. The PPVs for hallucinations were higher than for delusions, which is also in line with previous research ${ }^{10}$, possibly because questions about hallucinations may be less ambiguous than questions about delusions. Importantly, however, the current results also indicate that reporting a FP psychotic experience on a self-report measure may carry relevant risk-related psychometric information about an extended psychosis phenotype that is more likely to remain subclinical but may eventually also lead to need for care, as suggested by Bak and colleagues, who found that FP psychotic experiences predicted the subsequent development of psychotic disorder 3 years later ${ }^{11}$. The reported effect sizes may therefore point to a continuum of behavioral expression of risk, as suggested by Poulton and colleagues ${ }^{7}$. Indeed, probabilities of other psychopathology and environmental risk factors for psychotic disorder became successively higher with increasing certainty about the presence and nature of the reported experiences. The current study is an important addition to the NEMESIS-1 findings reported by Bak and colleagues as they found that FPs predicted future psychotic disorder but reported no additional characteristics of their FP group.

Compared with the FP group, the psychotic experiences of the TP group were more frequent and more recent. Furthermore, the TP group was about four times more likely to seek help for their psychotic experiences. This might represent the crucial difference between the FP and the TP group: the SRPEs were more likely to be confirmed by clinicians when they were more frequent and the individual had sought help for this experience. In a study by Brett and coworkers, evidence was found that underlying unhelpful metacognitive beliefs (that in turn were associated with anxiety and depression) were positively associated with help seeking for psychotic experiences ${ }^{32}$. In line with these findings, the present study showed that the TP group, compared with the FP group, more often had anxiety and mood disorder diagnoses, which could account for the increased help seeking behavior. 
The results of this study should be interpreted in the context of its strengths and limitations. Strengths are the size and representativeness of the sample. The most important limitation is the lack of information on whether the FP group also has a higher risk of transition to psychotic disorder compared with the control group. This information should become available as part of the ongoing follow-up of the current sample. A further limitation is that the clinical interviews were only conducted in those individuals reporting a possible psychotic experience and that the interview only included questions about these experiences. The consequence of this choice was that there was only information on FP experiences, not false negatives. Therefore, it was not possible to investigate the sensitivity and specificity of the various psychotic experiences. Another limitation was the chosen method of reinterviews: because it was not feasible to visit the participants with SRPEs on a second occasion for the purpose of a clinical interview, it was chosen to conduct the reinterview over the telephone. It is possible that subtle nonverbal cues have been missed that would have been picked up in a face-to-face interview. However, this method was also used in NEMESIS-1 and findings from these telephone interviews reliably predicted future psychotic disorder ${ }^{11}$, thus supporting its reliability.

In spite of these limitations, the present findings provide important clues about the characteristics of a subgroup of individuals presenting with experiences that may represent the mildest subthreshold expression of psychosis. Further study of this group may help to identify biological, psychological and social processes underlying the first expression of psychotic symptoms, the persistence of these over time and, eventually, development of need for care.

\section{Funding}

This work was supported by the Ministry of Health, Welfare and Sport (grant number 310253); with supplement support from the Netherlands Organization for Health Research and Development (ZonMw) and the Genetic Risk and Outcome of Psychosis (GROUP) investigators.

\section{Acknowledgements}

The Netherlands Mental Health Survey and Incidence Study-2 (NEMESIS-2) is conducted by the Netherlands Institute of Mental Health and Addiction (Trimbos Institute) in Utrecht. The reinterviews are conducted by the University of Maastricht, department of Psychiatry and Psychology. 


\section{REFERENCES}

1. Van Os J, Linscott RJ, Myin-Germeys I, Delespaul P, Krabbendam L. A systematic review and meta-analysis of the psychosis continuum: evidence for a psychosis-proneness-persistence-impairment model of psychotic disorder. Psychol Med 2009;39:179-195.

2. Dominguez MD, Wichers $M$, Lieb $R$, Wittchen $\mathrm{HU}$, van Os J. Evidence that onset of clinical psychosis is an outcome of progressively more persistent subclinical psychotic experiences: an 8-year cohort study. Schizophr Bull Jan 2011;37(1):84-93.

3. Dominguez MD, Saka MC, Lieb R, Wittchen $\mathrm{H}$, van Os J. Early expression of negative/disorganized symptoms predicting psychotic experiences and subsequent clinical psychosis: a 10-year study. Am J Psychiat 2010;167(9):1075-1082.

4. Tijssen MJA, van Os J, Wittchen $H U$, Lieb R, Beesdo K, Mengelers R, Wichers $M$. Prediction of transition from common adolescent bipolar experiences to bipolar disorder: a 10 year study. Brit J Psychiat 2010;196:102-108.

5. Bak M, Myin-Germeys I, Hanssen M, Bijl R, Vollebergh W, Delespaul P, van Os J. When does experience of psychosis result in need for care? A prospective general population study. Schizophr Bull 2003;29(2):349-358.

6. Werbeloff N, Drukker M, Dohrenwend $B P$, et al. Self-reported psychotic symptoms in the community are associated with increased risk of later hospitalization for non-affective psychotic disorders (conference abstract). Schizophr Bull 2009;35(suppl 1)(74).
7. Poulton R, Caspi A, Moffitt TE, Cannon M, Murray R, Harrington H. Children's self-reported psychotic symptoms and adult schizophreniform disorder: A 15 year longitudinal study. Arch Gen Psychiat 2000;57:1053-1058.

8. Linscott RJ, Van Os J. Systematic reviews of categorical versus continuum models in psychosis: evidence for discontinuous subpopulations underlying a psychometric continuum. Implications for DSM-V, DSM-VI, and DSM-VII. Annu Rev Clin Psycho 2010;6:391-419.

9. van Os J, Hanssen M, Bijl RV, Vollebergh W. Prevalence of psychotic disorder and community level of psychotic symptoms. Arch Gen Psychiat 2001;58:663-668.

10. Kelleher I, Harley M, Murtagh A, Cannon M. Are screening instruments valid for psychotic-like experiences? A validation study of screening questions for psychotic-like experiences using in-depth clinical interview. Schizophr Bull 2009;37(2):362-369.

11. Bak M, Delespaul P, Hanssen M, de Graaf R, Vollebergh W, van Os J. How false are "false" positive psychotic symptoms? Schizophr Res 2003;62:187-189.

12. van Os J, Kenis G, Rutten BP. The environment and schizophrenia. Nature Nov 11 2010;468(7321):203-212.

13. Bijl RV, van Zessen G, Ravelli A, de Rijk $C$, Langendoen Y. The Netherlands Mental Health Survey and Incidence Study (NEMESIS): Objectives and design Soc Psych Psych Epid 1998;33:581-586. 
14. De Graaf $R$, ten Have $M$, van Dorsselaer S. The Netherlands Mental Health Survey and Incidence study-2 (NEMESIS-2): design and methods. Int J Method Psych Res 2010;19(3):125-141.

15. De Graaf R, Ten Have M, van Gool C, Van Dorsselaer S. Prevalence of mental disorders and trends from 1996 to 2009. Results from the Netherlands Mental Health Survey and Incidence Study-2. Soc Psych Psych Epid;47(2):203-213.

16. Alonso J, Angermeyer M, Bernert $S$, et al. Sampling and methods of the European Study of the Epidemiology of Mental Disorders (ESEMeD) project. Acta Psychiat Scand 2004;109(Suppl. 420):8-20.

17. De Graaf R, Ormel J, Ten Have M, Burger $\mathrm{H}$, Buist-Bouwman M. Mental disorders and service use in the Netherlands. Results from the European Study of the Epidemiology of Mental Disorders (ESEMeD). The WHO World Mental Health Surveys: Global Perspectives on the Epidemiology of Mental Disorders. Kessler, R.C., Üstün, T.B. ed: Cambridge University Press; 2008:388-405.

18. Eysenck SBG, Eysenck HJ, Barrett P. Revised version of the psychoticism scale. Pers Indiv Differ 1985;6:21-29.

19. Eysenck SBG, White O, Eysenck HJ. Personality and mental illness. Psychol Rep 1976;39:1011-1022.

20. Van Os J, Bak M, Hanssen M, Bijl RV, De Graaf R, Verdoux H. Cannabis use and psychosis:alongitudinalpopulationbased study. Am J Epid 2002;156:319-327.
21. Stewart AL, Hayes RD, Ware JE. The MOS short form general health survey. Med Care 1988;26:724-735.

22. Ware JE, Sherbourne CD. The RAND36 Short-form Health status Survey: 1: Conceptual framework and item-selection. Med Care 1992;30:473-481.

23. Andrew G, Peters L. The psychometric properties of the Composite International Diagnostic Interview. Soc Psych Psych Epid 1998;33:80-88.

24. Wittchen HU. Reliability and validity studies of the WHO-Composite International Diagnostic Interview (CIDI): A critical review. J Psych Res 1994;28:57-84.

25. Helzer JE, Robins LN, McEvoy LT, Spitznagel EL, Stoltzman RK, Farmer A, Brockington IF. A comparison of clinical and diagnostic interview schedule diagnoses. Physician reexamination of lay-interviewed cases in the general population. Arch Gen Psychiatry 1985;42(7):657-666.

26. Cooper SA, Collacott RA. Clinical features and diagnostic criteria of depression in Down's syndrome. Br J Psychiatry 1994;165(3):399-403.

27. Krabbendam L, Myin-Germeys I, de Graaf R, Vollebergh W, Nolen WA, ledam J, van Os J. Dimensions of depression, mania and psychosis in the general population. Psychol Med 2004;34:1177-1186.

28. Spitzer RL, Williams JB, Gibbon M, First MB. The Structured Clinical Interview for DSM-III-R (SCID). I: History, rationale, and description. Arch Gen Psychiat 1992;49(8):624-629. 
29. Statistical Software: Release 10.0 [computer program]. Version. College Station, TX: Stata Corporation; 2007.

30. van Os J, Hanssen M, Bijl RV, Ravelli A. Strauss (1969) revisited: a psychosis continuum in the general population? Schizophr Res 2000;45:11-20.

31. Hanssen MSS, Bijl RV, Vollebergh W, van Os J. Self-reported psychotic experiences in the general population: a valid screening tool for DSMIII-R psychotic disorders? Acta Psychiat Scand 2003;107:369-377.

32. Brett CMC, Johns LC, Peters EP, McGuire PK. The role of metacognitive beliefs in determining the impact of anomalous experiences: a comparison of help-seeking and non-helpseeking groups of people experiencing psychotic-like anomalies.

Psychol Med 2009;39:939-950. 
36 | Surviving Childhood 


\section{CHAPTER 3}

\section{Evidence That Transition from Health to Psychotic Disorder Can Be Traced to Semi-Ubiquitous Environmental Effects Operating against Background Genetic Risk}

\section{Martine van Nierop ${ }^{\mathrm{D}, 1}$ \\ Mayke Janssens ${ }^{\mathrm{D}, 1}$ \\ GROUP Investigators}

Genetic Risk and OUtcome of Psychosis (GROUP) Investigators:

Richard Bruggeman ${ }^{A}$

Wiepke Cahn ${ }^{\mathrm{B}}$

Lieuwe de Haan ${ }^{\mathrm{C}}$

René S. Kahn ${ }^{B}$

Carin J. Meijer ${ }^{\mathrm{C}}$

Inez Myin-Germeys ${ }^{\mathrm{D}}$

Jim van Os*,DE

Durk Wiersma ${ }^{\mathrm{A}}$

${ }^{1}$ These authors contributed equally

A University Medical Center Groningen, Department of Psychiatry, Groningen, The Netherlands

B University Medical Center Utrecht, Department of Psychiatry, Rudolf Magnus Institute of Neuroscience, Utrecht, The Netherlands

C Academic Medical Centre, University of Amsterdam, Department of Psychiatry, Amsterdam The Netherlands

D Maastricht University Medical Centre, South Limburg Mental Health Research and Teaching Network, EURON, Maastricht, The Netherlands

E King's College London, King's Health Partners, Department of Psychosis Studies, Institute of Psychiatry, London, United Kingdom

PLOS ONE 2013; 8(11): e76690 


\section{ABSTRACT}

\section{Background}

In order to assess the importance of environmental and genetic risk on transition from health to psychotic disorder, a prospective study of individuals at average $(n=462)$ and high genetic risk $(n=810)$ was conducted.

\section{Method}

A three-year cohort study examined the rate of transition to psychotic disorder. Binary measures indexing environmental exposure (combining urban birth, cannabis use, ethnicity, and childhood trauma) and proxy genetic risk (high-risk sibling status) were used to model transition.

\section{Results}

The majority of high-risk siblings $(68 \%)$ and healthy comparison subjects $(60 \%)$ had been exposed to one or more environmental risks. The risk of transition in siblings ( $n=9,1.1 \%)$ was higher than the risk in healthy comparison subjects $\left(n=2,0.4 \%\right.$; $O R_{a d j}=2.2,95 \% \mathrm{Cl}: 0.5-$ 10.3). All transitions (100\%) were associated with environmental exposure, compared to $65 \%$ of non-transitions $(p=0.014)$, with the greatest effects for childhood trauma $\left(\mathrm{OR}_{\mathrm{adj}}=34.4,95 \% \mathrm{Cl}: 4.4-267.4\right)$, cannabis use (OR=4.1, 95\% Cl: 1.1, 15.4), minority ethnic group (OR=3.8, 95\% Cl: 1.2,12.8) and urban birth (OR=3.7, 95\% Cl: 0.9-15.4). The proportion of transitions in the population attributable to environmental and genetic risk ranged from $28 \%$ for minority ethnic group, $45 \%$ for urban birth, $57 \%$ for cannabis use, $86 \%$ for childhood trauma, and $50 \%$ for high-risk sibling status. Nine out of 11 transitions (82\%) were exposed to both genetic and environmental risk, compared to only $43 \%$ of non-transitions $(p=0.03)$.

\section{Conclusion}

Environmental risk associated with transition to psychotic disorder is semi-ubiquitous regardless of genetic high-risk status. Careful prospective documentation suggests most transitions can be attributed to powerful environmental effects that become detectable when analysed against elevated background genetic risk, indicating gene-environment interaction. 


\section{INTRODUCTION}

Several environmental factors have been implicated in the aetiology of psychotic illness including urban birth and upbringing ${ }^{1}$, minority position ${ }^{2}$, childhood trauma ${ }^{3}$ and cannabis $u^{4} e^{4}$. The impact of some environmental risk factors may be greater in those at increased genetic risk suggesting possible interaction between genetic and environmental risks ${ }^{5}$.

Associations between environment and mental illness typically are assessed in casecontrol studies that are easy to conduct but prone to bias and confounding. Cohort studies following individuals from health to illness transition are preferable but expensive and impractical given long incubation periods between exposure and outcome. In addition, given that the prevalence of psychotic disorder in the general population is low ${ }^{6}$, cohort studies require large samples that, in order to reduce costs, are subjected to inaccurate non-clinical diagnostic assessments. A high-risk cohort study, following individuals with higher than average genetic risk for psychotic disorder has advantages, given that transition rates will be higher, thus reducing required length of follow-up. In addition, by combining individuals of high average risk in the cohort, a proxy variable indexing genetic risk is created allowing for additional examination of genetic effects, as well as geneenvironment interaction ${ }^{7}$. There is also research on transition in individuals described at 'Ultra-High Risk' (UHR), however transition in this population does not refer to transition from health to psychotic disorder, as UHR samples in fact already are help-seeking patients with mental disorder diagnoses ${ }^{8}$ who present for treatment at mental health services ${ }^{9}$. In the current article, a detailed and careful follow-up was conducted of healthy siblings (of patients with non-affective psychotic disorder) and healthy comparison subjects sampled in the context of the GROUP study ${ }^{10}$, in order to determine true transitions from (nonpsychotic) health to psychotic disorder on the basis of clinical interview, and determine the contribution of genetic and environmental factors, taking into account the range of postnatal risk factors for which meta-analytic evidence exists ${ }^{5}$. Given these findings, we expect that urban birth, minority position, childhood trauma, and cannabis use all contribute to an increased probability of transition to psychotic disorder, and that this impact may be increased in individuals at higher than average genetic risk. 


\section{METHODS}

\section{Subjects}

Full details of the GROUP study have been presented elsewhere ${ }^{10,11}$. In representative geographical areas in the Netherlands and Belgium, patients were identified through clinicians working in regional psychotic disorder services, whose caseload was screened for inclusion criteria. Subsequently, a group of patients presenting at these services either as out-patients or in-patients were recruited for the study. Healthy comparison subjects were selected through random mailings to addresses in the catchment areas of the cases. The GROUP study was not conducted in a geographically well- defined small area, as it in fact included the majority of mental health services in the Netherlands, and a substantial part of mental health services in Dutch-speaking Belgium. Healthy comparison subjects could not be representative in all aspects, as an exclusion criterion was absence of a family history of psychotic disorder. The goal was to collect a control group that (i) was collected from the same geographical area as the case in the relevant mental health service, (ii) was sufficiently large to allow for chance variation and (iii) was frequency-matched in age- and sex distribution to the siblings and (iv) had absence of family history of psychotic disorder. Table 1 shows that healthy comparison subjects and siblings had similar sex distribution and also did not have large differences in age.

Table 1. Demographics of participants in the GROUP study

\begin{tabular}{|c|c|c|c|}
\hline \multirow[t]{2}{*}{ Variable } & Siblings ( $n=1057)$ & Healthy comparison subjects ( $n=589$ ) & \\
\hline & Mean (standard deviation) & Mean (standard deviation) & $t$ value $(p)$ \\
\hline Age at T0 & $27.8(8.3)$ & $30.4(10.6)$ & $5.53(<0.001)$ \\
\hline Gender, male (\%) & 45.6 & 45.7 & $-0.03(0.511)$ \\
\hline Education, Verhage $^{\mathrm{a}}$ & $5.1(2.1)$ & $5.4(1.8)$ & $3.26(0.001)$ \\
\hline WAIS-III Estimated IQ & $103.0(15.3)$ & $109.9(14.8)$ & $8.73(<0.001)$ \\
\hline Ethnicity, Caucasian (\%) & 83.2 & 92.0 & $4.98(<0.001)$ \\
\hline Urbanicity at birth & $2.7(1.7)$ & $2.6(1.7)$ & $-0.27(0.790)$ \\
\hline
\end{tabular}

aEducation (Verhage): range 0 (no education), 3-5 (school diploma) to 8 (university degree)

bUrbanicity: $1=<500 / \mathrm{km}^{2} ; 2=500-1000 / \mathrm{km}^{2} ; 3=1000-1500 / \mathrm{km}^{2} ; 4=1500-2500 / \mathrm{km}^{2} ; 5=2500+/ \mathrm{km}^{2}$

The full GROUP sample at baseline consisted of 1119 patients with non-affective psychotic disorder, 1057 siblings of these patients, 919 parents of the patients and 589 unrelated healthy comparison subjects. Inclusion criteria were: (i) age range 16 to 50 years and (ii) good command of Dutch language. For patients, an additional inclusion criterion was the presence of a clinical diagnosis of non-affective psychotic disorder. Healthy comparison subjects status was confirmed by using the Family Interview for Genetic studies ${ }^{12}$ with the control as informant, to establish absence of first degree relatives with a psychotic disorder. Diagnosis was based on the Diagnostic and Statistical Manual of Mental Disorder-IV (DSMIV) criteria ${ }^{13}$, assessed with the Comprehensive Assessment of Symptoms and History 
(CASH) interview ${ }^{14}$ or Schedules for Clinical Assessment for Neuropsychiatry (SCAN 2.1) . $^{15}$. The majority of patients had a DSM-IV diagnosis of schizophrenia (DSM-IV 295.x; $n=940$, $84 \%)$. In the sibling and control groups, there were respectively 151 (14\%) and 60 participants (10\%) with a history of a common mental disorder at baseline, the majority of who had a mood disorder (DSM-IV 296.x). For the purpose of the current analysis, the siblings and healthy comparison subjects groups were included.

The study was approved by the standing ethics committee (Medisch Ethische Toetsingscommissie, UMC Utrecht), and all the subjects gave written informed consent in accordance with the committee's guidelines. This committee waived the need for additional informed consent of parents or supervisors for underaged participants ages 16 and older, given the non-experimental/medical nature of this study.

\section{Substance use}

Substance use was assessed using the Composite International Diagnostic Interview (CIDI) ${ }^{16}$ and through urinalysis. Two different measures of cannabis exposure, assessed both at baseline and follow-up, were used to construct incident exposure to cannabis over the follow-up period: (i) CIDI lifetime cannabis use (hereafter: interview cannabis use): none (0), versus any use (1) and (ii) current cannabis use assessed by urinalysis (hereafter: urinalysis cannabis use): none (0) and present (1). Urinalysis was carried out as a screen for the presence of cannabis at the national Alcohol- and Drug use 'Jellinek' Laboratory. The method used was immunoassays with a cut-off of $50 \mathrm{ng} / \mathrm{ml}$. In addition, as an integrity parameter, the creatinine level of every sample was measured. Cannabis urine screening has a detection window up to 30 days, but the detection time has been documented in literature to be even longer (up to three months), depending on level of cannabis use ${ }^{17}$. Given the relatively high cut-off level of $50 \mathrm{ng} / \mathrm{ml}$, a conservative detection window of one month can be inferred. A dichotomous measure was created reflecting first exposure to cannabis over the follow-up period, defined as any instance of positive interview cannabis use or positive urinalysis cannabis use in those without interview cannabis use and without urinalysis cannabis use at baseline.

\section{Childhood trauma}

Childhood trauma was assessed with the Dutch version of the Childhood Trauma Questionnaire (CTQ) 25 item Short Form ${ }^{18}$, consisting of 25 items rated on a 5-point Likert scale (1=never to $5=$ very often). Emotional, physical and general abuse, and emotional and physical neglect were assessed, five items covering each trauma type ${ }^{18}$. Total trauma represents the mean score of all 25 items (range in siblings and healthy comparison subjects: $1-4.3$ ). Conform previous analyses in this sample ${ }^{19}$, trauma scores were dichotomized a priori into high trauma and low trauma, the cut-off being defined as the 80th percentile of scores for the healthy comparison subjects. 


\section{Urban birth}

Subjects were asked where they were born. To describe urbanicity, a historical population density record was generated for each municipality from 1930 onwards using the database of the Central Bureau of Statistics (Netherlands) and the HISSTAT database (University of Gent, Department Modern History, Belgium). When data was not available, linear extrapolations were computed. When historical names of municipalities disappeared from historical records (e.g. due to city mergers) available date from the agglomerate city were used. For each location, population density (by square kilometre, excluding water) at the municipality for that year was computed, on the basis of which the urbanicity code $\left(1=<500 / \mathrm{km}^{2} ; \quad 2=500-1000 / \mathrm{km}^{2} ; \quad 3=1000-1500 / \mathrm{km}^{2} ; \quad 4=1500-2500 / \mathrm{km}^{2} ; \quad 5=2500+/ \mathrm{km}^{2}\right)$ was calculated. In accordance with research using the five-level exposure, a binary urban birth exposure was calculated combining categories 1 to 3 (" 0 "), reflecting low urbanicity, and 4 and 5 ("1"), reflecting high urbanicity ${ }^{20,21}$.

\section{Psychosis measures}

The Community Assessment of Psychic Experiences (CAPE; www.cape42.homestead.com) was developed in order to rate self-reports of lifetime psychotic experiences. Items are modelled on patient experiences as contained in the PSE- $9^{22}$ and the schedules assessing negative symptoms such as the Scale for the Assessment of Negative Symptoms (SANS) ${ }^{23}$ and the Subjective Experience of Negative Symptoms (SENS) ${ }^{24}$. Items are scored on a 4-point scale. In the current analyses, CAPE dimensions of frequency of positive experiences (20 items) and negative experiences (14 items) were included (measured at baseline and 3-year follow-up), representing the person's perceived psychosis load over the lifetime (at baseline) or in the past three years (follow-up). A total score representing the mean of all items was calculated for each dimension (CAPE positive: range in siblings and comparison subjects: 0-2.5; CAPE negative: range 0-2.4).

\section{Other measures}

At baseline and at follow-up, the short form of the Wechsler Adult Intelligence Scale (WAIS) - III was assessed for an indication of intellectual functioning, and included the following tests: 'Block Design', 'Digit Symbol', 'Arithmetic', and 'Information'25,26. The WHOQOL-BREF ${ }^{27}$ was used at baseline and at follow-up to assess four domains of quality of life (1) physical health, (2) mental health, (3) social relationships, and (4) environment. At baseline, the Premorbid Adjustment Scale (PAS) ${ }^{28}$ was administered. The PAS is a rating scale that includes measures of social isolation, peer relationships, functioning outside of the family, and school functioning at 3 age periods (up to age 12 [4 items], 12-15 [5 items], 16-18 years [10 items]). Validity, interrater reliability and internal consistency have been found to be high ${ }^{29,30}$. An overall score based on the three age periods was created with a sample range from 0 (healthiest adjustment) to 5 (lowest adjustment). 


\section{Follow-up}

Healthy comparison subjects and siblings were eligible for follow-up. Of these, 78\% ( $n=1272$ ) were assessed at 3-year follow-up (healthy comparison subjects: $78 \%, n=462$; siblings: $77 \%, n=810$ ). Measures of cannabis use at follow-up reflected use over the interval between baseline and follow-up. Ratings of CASH, SCAN, SIS-R, and CAPE at follow-up reflected the period between baseline and follow-up. Mean follow-up was 3.3 years $(S D=0.5)$.

\section{Transition}

Transition from health to psychotic disorder was defined as (i) onset of non-affective psychotic disorder in individuals without psychotic disorder (DSMIV 295, 297, 298) and without psychotic affective disorder at baseline [n=11; 7 with 295, 3 with 298, 1 with documented psychotic illness who refused follow-up], (ii) onset of affective disorder or other non-psychotic disorder with evidence of psychotic symptoms rated of at least "considerable" or "severe" quality (or equivalent) on the CASH, PANSS or SCAN in individuals without baseline affective disorder or other non-psychotic disorder and without evidence of psychotic symptoms rated of at least "considerable" or "severe" quality at baseline $(n=0)$. Individuals who refused to be seen at follow-up were queried about mental health and contacts with mental health services. Participating relatives of refusing participants also provided information.

\section{Analysis}

Analyses were conducted using Stata, version $12^{31}$. Analyses focused on the siblings ( $n=1057$ at baseline and $n=810$ at follow-up) and healthy comparison subjects $(n=589$ at baseline, $n=462$ at follow-up). The dependent variable in the analyses was transition to psychotic disorder. Standard errors were corrected for hierarchical clustering of the data at the level of the family (clustering of siblings in the same family) or, when applicable, for clustering at the two levels occasioned by clustering of individuals in the same family and of repeated measures within the same person, using the Stata routines of cluster, xtreg or xtmixed, as appropriate.

Associations were expressed as the odds ratio from the logistic regression model (dichotomous transition outcome) or the regression coefficient (B) from multilevel random regression models (continuous variables). All analyses were a priori adjusted for age and sex. Comparisons between transition and non-transition status were additionally adjusted for sibling high risk status, in order to verify whether transition and non-transition differed in key variables independent of sibling high risk status.

In order to validate transitions, a comparison was made between transition and nontransition status with respect to key baseline variables as well as with respect to changes 
from baseline to follow-up. We thus expected that those who would make a transition to psychotic disorder would display more developmental impairment and higher levels of psychometric risk indicators at baseline (as measured with the CAPE, WHOQOL, PAS and WAIS). Differences in change from baseline to follow-up were examined in an xtmixed model of a repeated measure, whilst fitting an interaction between measurement occasion and transition status. Stratified associations were derived by linear combination from the model containing the interaction using the Stata margin command. The population attributable fraction associated with proxy environmental and genetic exposures was calculated using the cc command in Stata, and defined as the reduction in incidence that would be observed if the population were entirely unexposed, compared with its current exposure pattern.

\section{RESULTS}

\section{Sample and attrition}

At baseline, the risk set consisted of 589 healthy comparison subjects and 1057 siblings. Baseline demographic characteristics are shown in table 1 . Of these, respectively 462 (men: 44\%, mean age: 34.2 years, $s d=10.6$ ) and 810 (men: $44 \%$, mean age: 30.5 years, $\mathrm{sd}=7.9$ ) were seen at follow-up. Attrition was associated with male sex, urban environment and ethnic minority status, as well as with lower IQ and small differences in premorbid adjustment (Table 2). Attrition was not associated with age at baseline, CAPE positive or negative symptoms, cannabis use, childhood trauma and WHOQOL-BREF domains (small or non-significant differences; Table 2).

\section{Transition}

Those who made a transition to psychotic disorder were younger at baseline (transition: 22.9 years, $s d=4.8$; non-transition: 29.0 years, $s d=9.2$ years; $F=4.82, p=0.028$ ). Transition was not associated with $\operatorname{sex}(\mathrm{OR}=1.4,95 \% \mathrm{Cl}: 0.4-4.7)$. The 11 transitions were characterized by higher baseline psychopathology (CAPE positive and negative domains), poorer WHOQOL scores, lower IQ and poorer premorbid adjustment (Table 3). In addition, transitions also displayed greater increases in psychopathology from baseline to follow-up (CAPE positive and CAPE negative) and greater decreases in quality of life, with the exception of the environmental domain. Transition was not associated with changes in IQ (Table 3). One of the 11 individuals had a non-psychotic DSM-IV diagnosis at baseline (300.3 obsessivecompulsive disorder), in the absence of significant psychotic symptoms at interview. 
Table 2. Differences at baseline as a function of follow-up attrition

\begin{tabular}{|c|c|c|c|c|c|c|}
\hline & & Mean or \% & SD & $\mathrm{n}$ & F or $\chi^{2}$ & $\mathbf{p}$ \\
\hline \multirow[t]{2}{*}{ Age at baseline } & No follow-up & 28.1 & 9.6 & 374 & 2.4 & 0.126 \\
\hline & Follow-up & 28.9 & 9.2 & 1,272 & & \\
\hline \multirow[t]{2}{*}{ Male sex } & No follow-up & $51 \%$ & & 374 & 5.2 & 0.022 \\
\hline & Follow-up & $44 \%$ & & 1,272 & & \\
\hline \multirow[t]{2}{*}{ Minority ethnic group } & No follow-up & $24 \%$ & & 374 & 37.5 & $<0.001$ \\
\hline & Follow-up & $12 \%$ & & 1,272 & & \\
\hline \multirow[t]{2}{*}{ Urban birth } & No follow-up & $41 \%$ & & 326 & 8.5 & 0.004 \\
\hline & Follow-up & $32 \%$ & & 1,193 & & \\
\hline \multirow[t]{2}{*}{ Cannabis use } & No follow-up & $39 \%$ & & 369 & 0.4 & 0.547 \\
\hline & Follow-up & $37 \%$ & & 1,272 & & \\
\hline \multirow[t]{2}{*}{ Early Trauma } & No follow-up & $50 \%$ & & 137 & 0.2 & 0.654 \\
\hline & Follow-up & $52 \%$ & & 1,177 & & \\
\hline \multirow[t]{2}{*}{ CAPE positive } & No follow-up & 0.21 & 0.21 & 325 & 1.6 & 0.21 \\
\hline & Follow-up & 0.20 & 0.18 & 1,156 & & \\
\hline \multirow[t]{2}{*}{ CAPE negative } & No follow-up & 0.50 & 0.37 & 325 & 3.1 & 0.077 \\
\hline & Follow-up & 0.54 & 0.36 & 1,156 & & \\
\hline \multirow[t]{2}{*}{ WHOQOL physical } & No follow-up & 4.05 & 0.57 & 310 & 8.0 & 0.005 \\
\hline & Follow-up & 4.15 & 0.53 & 1,174 & & \\
\hline \multirow[t]{2}{*}{ WHOQOL mental } & No follow-up & 3.85 & 0.55 & 309 & 1.0 & 0.326 \\
\hline & Follow-up & 3.88 & 0.52 & 1,174 & & \\
\hline \multirow[t]{2}{*}{ WHOQOL social } & No follow-up & 3.86 & 0.76 & 309 & 1.2 & 0.282 \\
\hline & Follow-up & 3.91 & 0.65 & 1,174 & & \\
\hline \multirow[t]{2}{*}{ WHOQOL environmental } & No follow-up & 3.89 & 0.57 & 309 & 26.7 & $<0.001$ \\
\hline & Follow-up & 4.06 & 0.47 & 1,174 & & \\
\hline \multirow[t]{2}{*}{ IQ } & No follow-up & 101.0 & 14.1 & 342 & 37.6 & $<0.001$ \\
\hline & Follow-up & 106.7 & 15.7 & 1,236 & & \\
\hline \multirow[t]{2}{*}{ PAS premorbid adjustment } & No follow-up & 1.20 & 0.63 & 347 & 4.9 & 0.027 \\
\hline & Follow-up & 1.11 & 0.63 & 1,187 & & \\
\hline
\end{tabular}

\section{Environmental and genetic prediction of transition}

The majority of high-risk siblings (68\%) and healthy comparison subjects $(60 \%)$ had been exposed to one or more environmental risks. The risk of transition in siblings ( $n=9$ out of $810,1.1 \%$ ) was higher than the risk in healthy comparison subjects ( $n=2$ out of $462,0.4 \%$; OR adjusted for age and sex $=2.2,95 \% \mathrm{Cl}: 0.5-10.3$; Table 4). All transitions were associated with environmental exposure, compared to $65 \%$ of non-transitions $(p=0.014)$, with the greatest effects for childhood trauma (OR adjusted for age, sex and sibling status=34.4, $95 \% \mathrm{Cl}: 4.4-267.4)$, cannabis use (OR=4.1, 95\% Cl: 1.1, 15.4), minority ethnic group $(\mathrm{OR}=3.8,95 \% \mathrm{Cl}: 1.2,12.8)$ and urban birth $(\mathrm{OR}=3.7,95 \% \mathrm{Cl}: 0.9,15.4)$ (Table 3). 


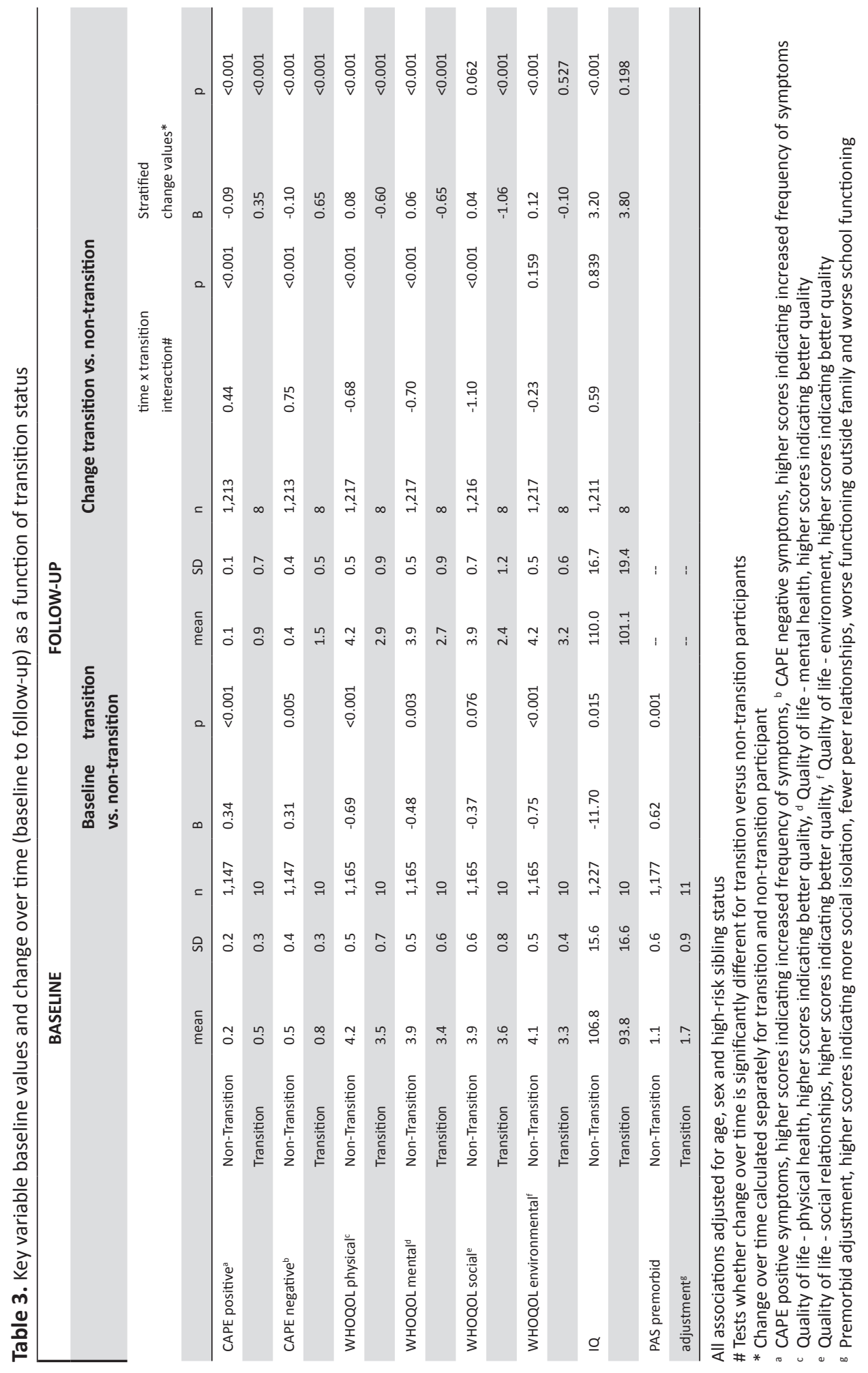


The proportion of transitions in the population attributable to environmental risk (PAF), assuming causality, ranged from $28 \%$ for minority ethnic group, $45 \%$ for urban birth, $57 \%$ for cannabis use, $86 \%$ for childhood trauma, and $50 \%$ for high-risk sibling status (Table 4). Nine out of 11 transitions (82\%) were exposed to both proxy genetic and environmental risk, compared to only $43 \%$ of non-transitions ( $p=0.03$; Table 5 ).

Table 4. Transition as a function of proxy environmental and genetic exposures

\begin{tabular}{|c|c|c|c|c|c|c|c|c|}
\hline & & \multicolumn{2}{|c|}{ Non transition } & \multicolumn{2}{|c|}{ Transition } & \multirow{2}{*}{$\begin{array}{l}\text { Odds } \\
\text { ratio }\end{array} *$} & \multirow[t]{2}{*}{$95 \% \mathrm{Cl}$} & \multirow[t]{2}{*}{ PAF\# } \\
\hline & & $\mathrm{n}$ & $\%$ & $\mathrm{n}$ & $\%$ & & & \\
\hline \multirow[t]{2}{*}{ Minority position } & Majority & 1,117 & 88.5 & 7 & 63.6 & 3.8 & $1.2-12.8$ & $28 \%$ \\
\hline & Minority & 145 & 11.5 & 4 & 36.4 & & & \\
\hline \multirow[t]{2}{*}{ Urban birth } & Non-urban & 807 & 68.0 & 3 & 32.0 & 3.7 & $0.9-15.4$ & $45 \%$ \\
\hline & Urban & 379 & 37.5 & 5 & 62.5 & & & \\
\hline \multirow[t]{2}{*}{ Cannabis use } & No use & 798 & 63.2 & 3 & 27.3 & 4.1 & $1.1-15.4$ & $57 \%$ \\
\hline & Use & 464 & 36.8 & 8 & 72.7 & & & \\
\hline \multirow[t]{2}{*}{ Early trauma } & No & 921 & 78.9 & 1 & 11.1 & 34.4 & $4.4-267.4$ & $86 \%$ \\
\hline & Yes & 247 & 21.2 & 8 & 88.9 & & & \\
\hline \multirow[t]{2}{*}{ Any exposure } & No & 447 & 35.4 & 0 & 0.0 & $\infty$ & & \\
\hline & Yes & 815 & 64.6 & 11 & 100.0 & & & \\
\hline \multirow[t]{2}{*}{ High risk group } & Comparison subject & 460 & 99.6 & 2 & 0.4 & 2.2 & $0.5-10.3$ & $50 \%$ \\
\hline & Sibling & 802 & 98.9 & 9 & 1.1 & & & \\
\hline
\end{tabular}

* Odd ratio's adjusted for age sex and high-risk sibling status

\# PAF=population attributable fraction, or the reduction in incidence that would be observed if the population were entirely unexposed, compared with its current exposure pattern

$\infty \quad=$ OR is infinity due to zero denominator

Table 5. Transition status as a function of exposure to proxy environmental (E) and/or genetic (G) exposures

\begin{tabular}{lllll}
\hline & & Neither G nor E & G or E & G and E \\
\hline Non-transition & $\mathrm{n}$ & 184 & 539 & 539 \\
& $\%$ & 14.6 & 42.7 & 42.7 \\
\multirow{3}{*}{ Transition } & $\mathrm{n}$ & 0 & 2 & 9 \\
& $\%$ & 0.0 & 18.2 & 81.8 \\
\hline
\end{tabular}

Pearson $\operatorname{chi}^{2}(2)=7.0 \mathrm{Pr}=0.030$ 


\section{DISCUSSION}

In order to assess the importance of environmental and genetic risk on transition from health to psychotic disorder, a prospective study of a cohort of individuals with average and high genetic risk was carried out. The findings suggest that the rate of exposure to any environmental risk in the population is very high, or semi-ubiquitous, and that transition from health to psychotic disorder is strongly dependent on such exposure. Thus, all environmental risk factors were associated with transition to psychotic disorder, with the greatest effect, in terms of both relative and attributable risk, for childhood trauma. Exposure to environmental risk did not vary as a function of genetic high risk status, suggesting absence of genetic control of environmental exposure, or gene-environment correlation. In those who made the transition to psychotic disorder, $82 \%$ were exposed to both proxy genetic and environmental risk, compared to only $43 \%$ of those who did not transition. This finding suggests that exposure to both genetic and environmental risk factors is necessary for transition, which is compatible with underlying gene-environment interaction. Careful prospective documentation therefore suggests most transitions can be attributed to powerful environmental effects operating against elevated background genetic risk.

\section{Incidence of transition}

Johnstone and colleagues followed a cohort of 163 young adults at average and high genetic risk, of which about $12 \%$ made a transition to psychotic disorder within 2.5 years ${ }^{7}$, representing a yearly transition rate of $4.9 \%^{7}$. The yearly transition rate in the current study was $0.34 \%$ for siblings ( $1.1 \%$ / 3.3 years of follow-up), and $0.13 \%$ for healthy comparison subjects ( $0.4 \%$ / 3.3 years of follow-up). Differences with the Edinburgh high risk study of Johnstone and colleagues ${ }^{32}$ may be related to the fact that their "high-risk" denoted more familial loading ( 2 affected relatives), and that the mean age was younger (21 years). The incidence in the healthy comparison subjects of the current study $(0.13 \%)$ appears high compared to the classic incidence estimate of schizophrenia $(0.02 \%)$. However, a direct comparison is not valid as our outcome included all affective and nonaffective psychosis, was restricted to a young age group and case identification did not depend on use of health care. Previous work has shown that the rate of psychosis, thus defined, is up to six times higher than typical estimates ${ }^{33}$.

The binary concept of transition may be difficult to define ${ }^{9}$. However, in the context of the current study, transition was from health to psychotic disorder, a clear and valid qualitative contrast that can be assessed reliably in the context of a clinical follow-up. The comparisons between transition and non-transition add to the notion of a valid contrast, given pretransition differences in premorbid adjustment and cognition, that did not further decline after onset, conform expectation ${ }^{34,35}$. However, the finding that cognition does not decline after onset has not been undisputed ${ }^{36}$. In addition, individuals developing psychotic disorder over the follow-up period displayed higher non-specific indices of psychometric risk and maladjustment as measured with the CAPE and WHOQOL at baseline, conform the model of clinical staging ${ }^{37}$. Furthermore, transition resulted in substantial increases for these variables, indicating true clinical change. 


\section{Comparison with previous work}

The results are in line with previous research showing associations between several environmental risk factors and development of psychotic symptoms or psychotic disorder ${ }^{1-4}$, particularly in those at high risk ${ }^{5}$. As "high-risk" in the current study was defined on the basis of higher than average genetic risk, rather that psychometric risk as observed in UHR samples $^{38}$ or samples with attenuated psychotic symptoms in the general population ${ }^{39}$, comparison with previous work is limited. Both Habets and colleagues ${ }^{40}$, as well as Welch and colleagues ${ }^{41}$ showed that cannabis use was associated with differential impact on brain structures in individuals at familial high risk for schizophrenia, which Habets and colleagues furthermore did not observe in controls. Similarly, epidemiological studies have demonstrated that the impact of urbanicity on schizophrenia risk is greater in those with additional evidence of elevated genetic risk ${ }^{21,42,43}$.

\section{Genetic risk and environment risk: ubiquitous?}

Studies focussing on the nature and extent of molecular genetic risk for schizophrenia have provided "molecular genetic evidence for a substantial polygenic component to the risk of schizophrenia involving thousands of common alleles of very small effect" 44 . In other words, molecular genetic variation contributing to risk for schizophrenia can be considered ubiquitous and distributed. Interestingly, the current study, being one of the first to examine multiple environmental risks together, suggests that conceptually the situation with regard to environmental risks may be similar. Thus, most individuals in the population were exposed to one or more of the environmental risks included in this study, and most of the transitions were attributable to environmental risk factors, against a background of genetic risk (most of the transitions being siblings of higher than average genetic risk). Methodologically this is an important issue, as the impact of a risk factor on a disease outcome cannot be detected if the entire population is exposed, unless the population can be separated into those who are differentially susceptible. Given the very high rate of exposure to environmental risks, the results suggest that careful follow-up of samples of differential genetic risk for psychotic disorder may be necessary to examine the true impact of environmental risk factors.

The nature of the impact of the environmental risks examined in the current study requires further clarification. First, the sample was too small to examine to what degree the environmental risks acted additively or more-than-additively. Previous work in general populations samples suggest that relationships may be both additive ${ }^{45}$ and more-thanadditive $^{46,47}$. In addition, the focus was on postnatal risk factors, although pre-natal risks may also play an important role $48-51$.

Second, the data are not informative as to when and how the environmental factors examined impact on development to increase risk, and whether environmental risks gave rise to enduring liability early in life, or acted as precipitants in individuals at higher than average genetic risk. The temporal focus of the current investigation was on transition from health to illness, and retrospective examination of environmental impact is methodologically challenging. 
A remarkable finding was the very high relative and attributable risk associated with childhood trauma. Given the prospective nature of the investigation, bias associated with a "search for meaning" cannot explain the results, in agreement with a growing number of prospective analyses testing the relationship between childhood adversity on the one hand, and psychosis on the other ${ }^{52-56}$. The results confirm the need to urgently identify the nature and the mechanism of risk associated with early adversity, as well the clinical implications thereof ${ }^{57}$.

\section{Methodological issues}

Strengths of the study include careful prospective assessment and confirmation of control status by excluding those with a positive family history. Because of the relatively short follow-up period, the number of individuals in this study who transitioned to psychotic disorder was relatively small. Although some results were statistically conclusive, other analyses, for example risk associated with sibling status, were underpowered. As the sample will be seen again at six-year follow-up, amplification of the sample and more robust results will be possible, as well as more fine-grained testing of relationships between genetic and non-genetic risks. Another issue is selection, as the focus was on transition in siblings and healthy comparison subjects who had lived through a substantial period of risk. It cannot be excluded that the mix of risk factors impacting on transition varies as a function of age-at-onset, thus the results cannot necessarily be generalised to transitions from health to illness at all ages.

\section{Acknowledgements:}

We are grateful for the generosity of time and effort by the families who make the GROUP project possible. 


\section{REFERENCES}

1. Vassos E, Pedersen CB, Murray R, Collier DA, Lewis CM. Meta-analysis of the association of urbanicity with schizophrenia. Schizophr Bull 2012;38(6):1118-1123.

2. Cantor-Graae E, Selten JP. Schizophrenia and migration: a meta-analysis and review. Am J Psychiat 2005;162:12-24.

3. Varese F, Smeets F, Drukker M, et al. Childhood adversities increase the risk of psychosis: a meta-analysis of patient-control, prospective- and cross-sectional cohort studies. Schizophr Bull 2012;38(4):661-671.

4. Matheson SL, Shepherd AM, Laurens KR, Carr VJ. A systematic meta-review grading the evidence for non-genetic risk factors and putative antecedents of schizophrenia. Schizophr Res Dec 2011;133(1-3):133-142.

5. van Os J, Kenis G, Rutten BP. The environment and schizophrenia. Nature Nov 11 2010;468(7321): 203-212.

6. van Nierop M, Van Os J, Gunther N, et al. Phenotypically continuous with clinical psychosis, discontinuous in need for care: Evidence for an extended psychosis phenotype. Schizophr Bull 2012;38(2):231-238.

7. Johnstone EC, Ebmeier KP, Miller P, Owens DG, Lawrie SM. Predicting schizophrenia: findings from the Edinburgh High-Risk Study.

Br J Psychiat Jan 2005;186:18-25.

8. Fusar-Poli P, Nelson B, Valmaggia L, Yung AR, McGuire PK. Comorbid Depressive and Anxiety Disorders in 509 Individuals With an At-Risk Mental State: Impact on Psychopathology and Transition to Psychosis.
Schizophr Bull 2012;doi:10.1093/ schbul/sbs136.

9. Fusar-Poli P, van Os J. Lost in transition: setting the psychosis threshold in prodromal research. Acta Psychiat Scand 2013;127(3):248-252.

10. G.R.O.U.P. Evidence that familial liability for psychosis is expressed as differential sensitivity to cannabis: an analysis of patient-sibling and sibling-control pairs. Arch Gen Psychiat 2011;68(2):138-147.

11. Korver N, Quee PJ, Boos HB, Simons C, de Haan L. Genetic Risk and Outcome of Psychosis (GROUP), a multi site longitudinal cohort study focused on gene-environment interaction: objectives, sample characteristics, recruitment and assessment methods. Int J Method Psych 2012;21(3):205-221.

12. NIMH.Genetics.Initiative. Family Interview for Genetic Studies (FIGS). Rockville, Md: National Institute of Mental Health; 1992.

13. American Psychiatric Association. Diagnostic and statistical manual of mental disorders. 4th ed., text rev. ed: Washington, DC; 2000.

14. Andreasen NC, Flaum M, Arndt S. The Comprehensive Assessment of Symptoms and History (CASH). An instrument for assessing diagnosis and psychopathology. Arch Gen Psychiat 1992;49(8):615-623.

15. Wing JK, Babor T, Brugha $\mathrm{T}$, et al. SCAN. Schedules for Clinical Assessment in Neuropsychiatry. Arch Gen Psychiat 1990;47(6):589-593.

16. World Health Organisation. Composite International Diagnostic Interview (CIDI) Version 1.0. Geneva: World Health Organisation; 1990. 
17. Musshoff F, Madea B. Review of biologic matrices (urine, blood, hair) as indicators of recent or ongoing cannabis use. Ther Drug Monit Apr 2006;28(2):155-163.

18. Bernstein DP, Ahluvalia T, Pogge $D$, Handelsman L. Validity of the Childhood Trauma Questionnaire in an adolescent psychiatric population.

J Am Acad Child Psy 1997;36(3):340-348.

19. Heins $M$, Simons $C$, Lataster $T$, et al. Childhood trauma and psychosis:

A case-control and case-sibling comparison across different levels of genetic liability, psychopathology, and type of trauma. Am J Psychiat 2011;168(12):1286-1294.

20. Marcelis M, Takei N, Van Os J. Urbanization and risk for schizophrenia: does the effect operate before or around the time of illness onset? Psychol Med Sep 1999;29(5):11971203.

21. Van Os J, Hanssen M, Bak M, Bijl RV, Vollebergh W. Do urbanicity and familial liability coparticipate in causing psychosis? Am J Psychiat Mar 2003;160(3):477-482.

22. Wing JK, Cooper JE, Sartorius N. The measurement and classification of psychiatric symptoms. London: Cambridge University Press; 1974.

23. Andreasen NC. Negative symptoms in schizophrenia. Definition and reliability. Arch Gen Psychiat 1982;39(7):784-788.

24. Selten JP, Sijben NE, van den Bosch RJ, Omloo Visser J, Warmerdam $\mathrm{H}$. The subjective experience of negative symptoms: a self-rating scale.

Compr Psychiatry 1993;34(3):192-197.
25. Blyler CR, Gold JM, lannone VN, Buchanan RW. Short form of the WAIS-III for use with patients with schizophrenia. Schizophr Res Dec 15 2000;46(2-3):209-215.

26. Velthorst $E$, Levine $S Z$, Henquet $C$, de Haan L, van Os J, Myin-Germeys I, Reichenberg A. To cut a short test even shorter: Reliability and validity of a brief assessment of intellectual ability in Schizophrenia-a control-case family study. Cogn Neuropsychiatry Nov 202012.

27. The WHOQOL Group. Development of the World Health Organization WHOQOL-BREF quality of life assessment. Psychol Med 1998;28:551-558.

28. Cannon Spoor HE, Potkin SG, Wyatt RJ. Measurement of premorbid adjustment in chronic schizophrenia. Schizophr Bull 1982;8(3):470-484.

29. Brill N, Reichenberg A, Weiser M, Rabinowitz J. Validity of the premorbid adjustment scale. Schizophr Bull 2008;34(5):981-983.

30. Small NE, Mohs RC, Halperin R, Rosen WG, Masterson C, Kendler KS, Horvath TB, Davis KL. A study of the reliability of reported premorbid adjustment in schizophrenic patients. Biol Psychiatry 1984;19(2):203-211.

31. StataCorp. STATA Statistical Software: Release 12. Texas: College Station; 2011.

32. Johnstone EC, Ebmeier KP, Miller P, Owens DGC, Lawrie SM. Predicting schizophrenia: findings from the Edinburgh High-Risk Study. Brit J Psychiat 2005;186:18-25. 
33. Perala J, Suvisaari J, Saarni SI, et al. Lifetime prevalence of psychotic and bipolar I disorders in a general population.Arch Gen Psychiat Jan 2007;64(1):19-28.

34. Heaton RK, Gladsjo JA, Palmer BW, Kuck J, Marcotte TD, Jeste DV. Stability and course of neuropsychological deficits in schizophrenia. Arch Gen Psychiat Jan 2001;58(1):24-32.

35. Russell AJ, Munro JC, Jones PB, Hemsley DR, Murray RM.

Schizophrenia and the myth of intellectual decline. Am J Psychiat 1997;154(5):635-639.

36. Kahn RS, Keefe RSE. Schizophrenia is a cognitive illness; time for a change in focus. JAMA in press.

37. McGorry P, Van Os J. Redeeming Diagnosis in Psychiatry: Timing versus Specificity Lancet 2013;381:343-345.

38. Fusar-Poli P, Bonoldi I, Yung A, et al. Predicting psychosis: meta-analysis of transition outcomes in individuals at high clinical risk. Arch Gen Psychiat 2012;69(3):220-229.

39. Kaymaz N, Drukker M, Lieb R, Wittchen $\mathrm{H}$, Werbeloff $\mathrm{N}$, Weiser M, Lataster T, Van Os J. Do subthreshold psychotic experiences predict clinical outcomes in unselected non-helpseeking population-based samples? A systematic review and meta-analysis, enriched with new results.

Psychol Med 2012;DOI:10.1017/ S0033291711002911.

40. Habets P, Marcelis M, E. G, Drukker $M$, van Os J. Reduced cortical thickness as an outcome of differential sensitivity to environmental risks in schizophrenia. Biol Psychiatry 2011;69(5):487-494.
41. Welch KA, Stanfield AC, McIntosh AM, et al. Impact of cannabis use on thalamic volume in people at familial high risk of schizophrenia. $\mathrm{Br} J$ Psychiat Nov 2011;199(5):386-390.

42. van Os J, Pedersen CB, Mortensen PB. Confirmation of synergy between urbanicity and familial liability in the causation of psychosis. Am J Psychiat Dec 2004;161(12):2312-2314.

43. Weiser M, van Os J, Reichenberg A, et al. Social and cognitive functioning, urbanicity and risk for schizophrenia. Br J Psychiat Oct 2007;191:320-324.

44. Purcell SM, Wray NR, Stone JL, et al. Common polygenic variation contributes to risk of schizophrenia and bipolar disorder. Nature Jul 12009.

45. Cougnard A, Marcelis M, MyinGermeys I, et al. Does normal developmental expression of psychosis combine with environmental risk to cause persistence of psychosis? A psychosis proneness-persistence model. Psychol Med Apr 2007;37(4):513-527.

46. Harley M, Kelleher I, Clarke M, Lynch F, Arseneault L, Connor D, Fitzpatrick C, Cannon M. Cannabis use and childhood trauma interact additively to increase the risk of psychotic symptoms in adolescence. Psychol Med Oct 2010;40(10):1627-1634.

47. Clarke MC, Tanskanen A, Huttunen M, Whittaker JC, Cannon M. Evidence for an interaction between familial liability and prenatal exposure to infection in the causation of schizophrenia. Am J Psychiat Sep 2009;166(9):1025-1030. 
48. McGrath J, Burne TH, Feron F, Mackay-Sim A, Eyles DW.

Developmental vitamin $D$ deficiency and risk of schizophrenia: a 10-year update.SchizophrBull2010;36(6):10731078.

49. Cannon M, Jones PB, Murray RM. Obstetric complications and schizophrenia: historical and meta-analytic review.AmJPsychiat2002;159(7):10801092.

50. Brown AS. Epidemiologic studies of exposure to prenatal infection and risk of schizophrenia and autism. Dev Neurobiol 2012;72(10):1272-1276.

51. Kirkbride JB, Susser E, Kandakovic M, Kresovich JK, Davey Smith G, Relton $\mathrm{CL}$. Prenatal nutrition, epigenetics and schizophrenia risk: can we test causal effects? Epigenomics 2012;4(3):303315.

52. Wicks S, Hjern A, Gunnell D, Lewis G, Dalman C. Social adversity in childhood and the risk of developing psychosis: a national cohort study. Am J Psychiat 2005;162:1652-1657.

53. Janssen I, Krabbendam L, Bak M, Hanssen M, Vollebergh W, De Graaf R, Van Os J. Childhood abuse as a risk factor for psychotic experiences. Acta Psychiat Scand 2004;109:38-45.
54. Elklit A, Shevlin M. Female Sexual Victimization Predicts Psychosis:

A Case-Control Study Based on the Danish Registry System. Schizophr Bull 2011;37(6):1305-1310.

55. Schreier A, Wolke $D$, Thomas $K$, et al. Prospective study of peer victimization in childhood and psychotic symptoms in a nonclinical population at age 12 years. Arch Gen Psychiat 2009;66(5):527-536.

56. Cutajar MC, Mullen PE, Ogloff JRP, Thomas SD, Wells DL, Spataro J. Schizophrenia and other psychotic disorders in a cohort of sexually abused children. Arch Gen Psychiat 2010;67(11):1114-1119.

57. Read J, Bentall RP. Negative childhood experiences and mental health: theoretical, clinical and primary prevention implications. Br J Psychiat Feb 2012;200(2):89-91. 


\title{
CHAPTER 4
}

\section{Psychopathological Mechanisms Linking Childhood Traumatic Experiences to Risk of Psychotic Symptoms: Analysis of a Large, Representative Popu- lation-Based Sample}

\author{
Martine van Nierop ${ }^{A}$ \\ Tineke Lataster ${ }^{A}$ \\ Feikje Smeets ${ }^{A}$ \\ Nicole Gunther ${ }^{c}$ \\ Catherine van Zelst ${ }^{A}$ \\ Ron de Graaf ${ }^{\mathrm{D}}$ \\ Margreet ten Have ${ }^{\mathrm{D}}$ \\ Saskia van Dorsselaer ${ }^{\mathrm{D}}$ \\ Maarten Bak ${ }^{\mathrm{A}}$ \\ Inez Myin-Germeys ${ }^{A}$ \\ Wolfgang Viechtbauer ${ }^{A}$ \\ Jim van $O s^{A B}$ \\ Ruud van Winkel ${ }^{A E}$
}

\footnotetext{
A Dept. of Psychiatry and Psychology, School for Mental Health and Neuroscience, EURON, Maastricht University Medical Centre, PO Box 616 (Vijv1), 6200 MD Maastricht, The Netherlands

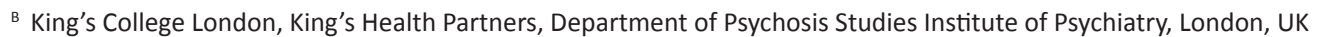

c School of Psychology, Open University, The Netherlands

${ }^{D}$ Netherlands Institute of Mental Health and Addiction, Da Costakade 45, 3521 VS Utrecht, The Netherlands

E University Psychiatric Center Katholieke Universiteit Leuven, campus Kortenberg, Leuvensesteenweg, Kortenberg, Belgium
} 


\section{ABSTRACT}

\section{Background}

Different psychological models of trauma-induced psychosis have been postulated, often based on the observation of 'specific' associations between particular types of childhood trauma (CT) and particular psychotic symptoms, or the co-occurrence of delusions and hallucinations. However, the actual specificity of these associations remains to be tested.

\section{Methods}

In two population-based studies with comparable methodology (NEMESIS-1 and NEMESIS-2, N=13722), trained interviewers assessed $C T$, psychotic symptoms and other psychopathology. Specificity of associations was assessed with mixed-effects regression models with multiple outcomes, a statistical method suitable to examine specificity of associations in case of multiple correlated outcomes.

\section{Results}

Associations with $\mathrm{CT}$ were strong and significant across the entire range of psychotic symptoms, without evidence for specificity in the relationship between particular trauma variables and particular psychotic experiences (PE). Abuse and neglect were both associated with PEs (OddsRatio [OR $]_{\text {abuse }} 2.12, p<0.001 ; \mathrm{OR}_{\text {neglect }} 1.96, \mathrm{p}<0.001$ ), with no large or significant difference in effect size. Intention-to-harm experiences showed stronger associations with psychosis than CT without intent $\left(\chi^{2} 58.62, p<0.001\right)$. Most trauma variables increased the likelihood of co-occurrence of delusions and hallucinations rather than either symptom in isolation.

\section{Discussion}

Intention-to-harm is the key component linking childhood traumatic experiences to psychosis, most likely characterized by co-occurrence of hallucinations and delusions, indicating buildup of psychotic intensification, rather than specific psychotic symptoms in isolation. No evidence was found to support psychological theories regarding specific associations between particular types of CT and particular psychotic symptoms. 


\section{INTRODUCTION}

Childhood trauma (CT) has been studied extensively as a risk factor for psychosis ${ }^{1}$. In a comprehensive meta-analysis, a positive association with an overall odds ratio of 2.78 was found ${ }^{1}$. However, knowledge of possible underlying mechanisms is still limited. Several authors, in an attempt to interpret epidemiological data, have postulated different psychological theories linking childhood traumatic experiences to later development of psychosis.

For example, it has been proposed that CT results in post-traumatic reactions later in life, expressed as hallucinations ${ }^{2}$. A related theory is that delusions may be a cognitive effort to make sense of hallucinatory experiences ${ }^{3}$, which may be more likely to occur in individuals who have experienced $\mathrm{CT}$, as a study in a large sample of adolescents and young adults recently found that co-occurrence of hallucinations and delusions, compared to occurrence of either symptom in isolation, was more likely in individuals exposed to trauma ${ }^{4}$.

In addition to studies examining differences in the phenotypic expression of psychosis associated with CT, a number of studies have focused on the possible influence of the type of trauma experienced, using subdivisions of $\mathrm{CT}$, i.e. abuse and neglect ${ }^{5,6}$. These studies reported stronger associations of abuse and psychosis, compared with neglect ${ }^{5,6}$. Other authors focused on the possible influence of particular traumatic experiences on specific psychotic symptoms such as auditory-verbal hallucinations (AVH) and paranoia ${ }^{7}$, based on the hypothesis that sexual abuse may specifically impair source monitoring, thus inducing $\mathrm{AVH}$, whereas growing up outside a family setting (i.e. foster care) may impact on attachment styles, thus predisposing to paranoia ${ }^{7}$.

Lastly, some studies examined whether the intentional nature of the traumatic experience is an important factor, in agreement with the 'social defeat' theory of psychosis ${ }^{8}$. These studies suggested that the possible effect of accidents during childhood, compared to childhood maltreatment, may be smaller ${ }^{9}$.

A hitherto neglected point is that examination of the question whether particular trauma variables are specifically associated with particular types of psychotic symptoms, requires the use of specialized statistical models. The observation of greater effect sizes of particular trauma variables on particular psychotic symptoms $\mathrm{s}^{4-7}$, or the finding of a significant association with one type of trauma but not another ${ }^{5,9}$, does not necessarily imply that there is a 'specific' association that validates a specific etiopathogenic model ${ }^{10}$. In fact, the examination of possible specificity of associations in case of multiple correlated outcomes (such as hallucinations and delusions) requires the use of specific mixed-effects regression models with multiple outcomes, which have not been used previously ${ }^{10}$. 
The aim of the present study was to investigate epidemiological evidence underlying the aforementioned psychological theories linking trauma and psychotic experiences (PE), using mixed-effects regression models with multiple outcomes where applicable, in a combined sample of Dutch individuals from the general population $(N=13722)$, consisting of two population-based samples with comparable methodology (NEMESIS-1, N=7076 and NEMESIS-2, $\mathrm{N}=6646$ ). 


\section{METHODS}

This study is part of the first and second Netherlands Mental Health Survey and Incidence Study (NEMESIS-1 and NEMESIS-2), two longitudinal studies on the prevalence and incidence of psychiatric disorders in the Dutch general population. Both studies were approved by a medical ethics committee and respondents provided written informed consent. The participants were interviewed at home by trained interviewers, who were not clinicians, with the Composite International Diagnostic Interview (CIDI) version $1.1^{11}$ (NEMESIS-1) and 3.012 (NEMESIS-2), and additional questionnaires. A more detailed description of NEMESIS- $1^{13}$ and NEMESIS- $2^{14}$ is presented elsewhere.

\section{Childhood trauma}

CT was assessed using a questionnaire developed for NEMESIS- $1^{13}$. Whenever a subject reported having experienced one of four types of CT (emotional neglect [ignored or unsupported], physical abuse [kicked, hit, bitten or hurt], psychological abuse [yelled at, insulted or threatened] and sexual abuse [any unwanted sexual experience]) before the age of 16 , they were asked to state how often it had occurred, on a scale of 1 (once) to 5 (very often). As these trauma scales loaded strongly onto one factor (eigenvalue 2.56), an overall trauma score was made by adding the scores of each trauma type (range 0-20). Furthermore, subjects were asked about upbringing in a foster family, and whether during childhood a parent, sibling or close friend had died. As these trauma variables were binary, they were not included in the total trauma score.

\section{Psychosis}

Studies with earlier versions of the CIDI concluded that the instrument provides a reliable and valid assessment of mental disorders, with the exception of psychotic disorders ${ }^{15}$. Thus, a psychosis add-on instrument was constructed, based on the section of psychotic symptoms in CIDI version 1.1. This part of the interview consisted of questions regarding 20 lifetime PE, each rated 'yes' or 'no' (see van Nierop et al. ${ }^{16}$ for a detailed description).

Individuals who endorsed at least one lifetime PE were contacted for reinterview over the telephone by an experienced clinician. Reinterviews were conducted using questions from the Structured Clinical Interview for DSM-IV (SCID-I), an instrument with proven reliability and validity ${ }^{17}$. Self-reported PE were considered present when the subject reported a PE at the first (lay) interview; validated PEs were considered present if the selfreported PEs were confirmed at the follow-up interview. In NEMESIS-2, all self-reported PEs were followed-up for validation ${ }^{16}$. In NEMESIS-1, the lay-interviewers scored each (self-reported) PE on a scale of 1-6: ' 1 ', no symptom; ' 2 ', PE present but not clinically relevant; ' 3 ', PE result of drug use; ' 4 ', PE result of somatic disease; ' 5 ', true PE; ' 6 ', possible plausible explanation for what appears to be a PE. Each participant with a score of either 2,5 or 6 score was followed up for validation ${ }^{18}$. 


\section{Depression and cannabis}

Consistent with earlier work in NEMESIS- $1^{19}$ and other CIDI-based population work ${ }^{20}$, a lifetime depression score was obtained by adding the ratings of the 28 symptom items (present/not present) from the CIDI 1.1 or 3.0 Depression section. Cannabis use was assessed in the section Illegal Substance Use of the CIDI 1.1 or CIDI 3.0. Conform previous work in NEMESIS, 'cannabis use' was defined as use of cannabis a least once in the lifetime ${ }^{21}$.

\section{Sample and prevalences}

The NEMESIS-1 sample consisted of 7076 participants (response rate $70 \%$ ), see Bijl et al. ${ }^{13}$ and Table 1 for characteristics. Of the 479 participants eligible for reinterview, 226 participants (47\%) were interviewed. The NEMESIS-2 sample consisted of 6646 participants (response rate 65\%; see Table 1 for characteristics). Of the 1078 participants eligible for re-interview, 792 participants were interviewed $(74 \%)$. 


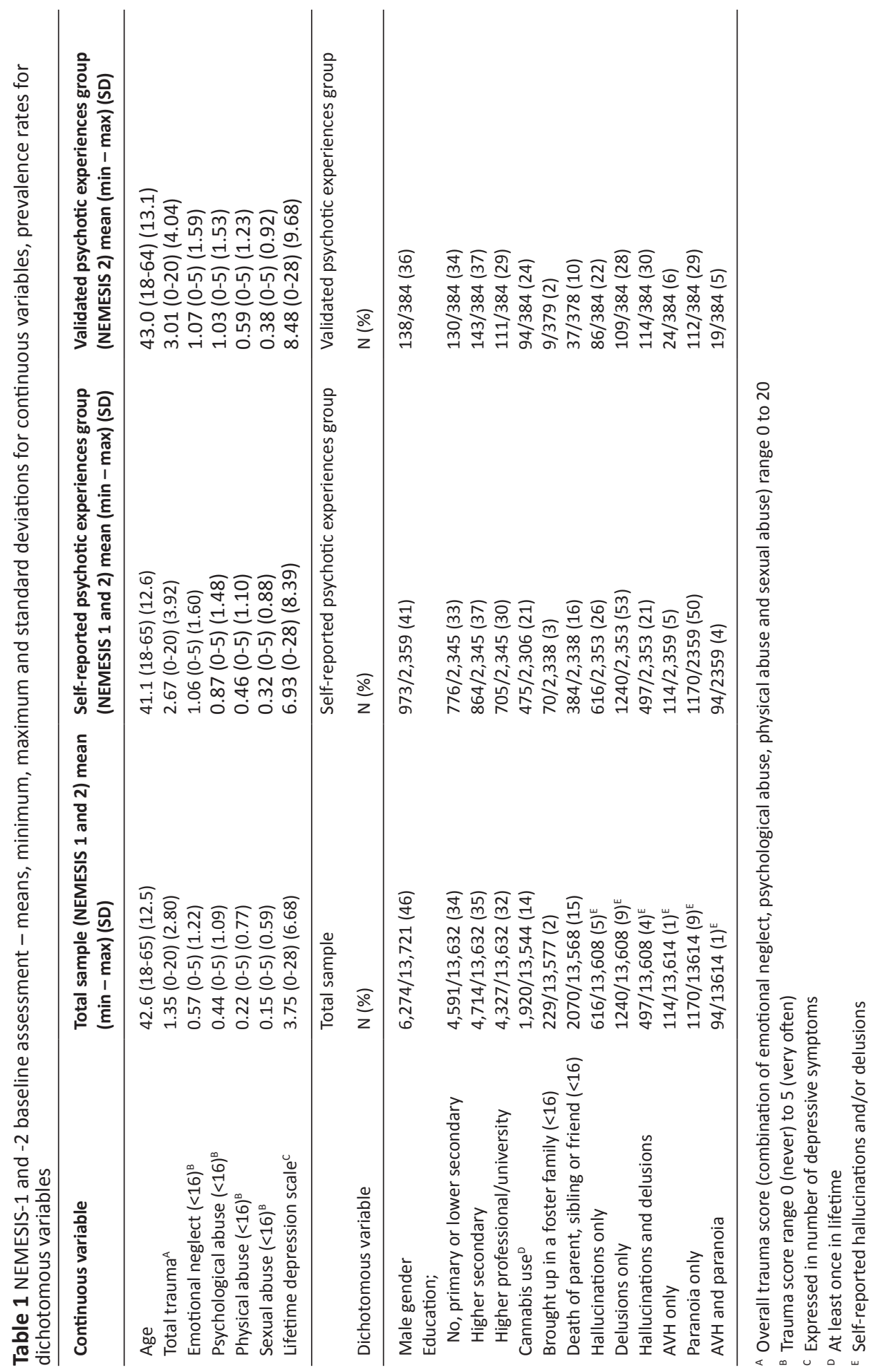




\section{Analyses}

All analyses were performed using Stata, version $11^{22}$. Each type of analysis was first performed in the full sample (NEMESIS- 1 and NEMESIS- 2 combined) using self-reported PES as a measure of psychosis. However, since self-report of psychotic symptoms is known to yield 'false-positives' ${ }^{\prime 16}$, additional sensitivity analyses were conducted in the subsample with confirmed interview-based PEs. In order to avoid issues concerning differences in methodology between NEMESIS-1 and NEMESIS-2 with regard to the validation of selfreported PEs, for the sensitivity analyses validated PEs were taken from the NEMESIS-2 sample only. Furthermore, given the amount of multiple, related hypotheses involved, the threshold of statistical significance was set at $p<0.001$. A priori confounders included in all analyses were depression ${ }^{23}$, cannabis use ${ }^{24}$, age and gender.

\section{Hallucinations, delusions, AVH and paranoia}

Associations between all trauma variables and occurrence of any hallucinations, delusions, $\mathrm{AVH}$ (hearing voices) or paranoia (persecutory ideation, fear of being spied on, secretly being tested on or being the victim of a conspiracy) were first established using logistic regression with hallucinations, delusions, AVH or paranoia as dependent variables. Regression analyses were carried out for each type of symptom and type of trauma separately.

In order to examine whether CT increases the likelihood of hallucinations and delusions co-occurring together, multinomial logistic regression was applied using type and combination of PEs (no PE [reference group], isolated hallucinations, isolated delusions, or combination of hallucinations and delusions) as the dependent variable. Then, associations of trauma with a combination of symptoms versus isolated symptoms was assessed using post hoc analyses by Wald test ${ }^{25}$. A sensitivity analysis in the NEMESIS-2 subsample was impossible due to small group sizes (Table 1 ).

\section{Specificity of associations between 'type of trauma' and either hallucinations or delusions, and AVH or paranoia}

Analyses examined whether any of the trauma variables had a stronger association with either hallucinations or delusions, by performing a mixed-effects logistic regression model (XTMELOGIT command in Stata). The multilevel models used for the analyses allow for a proper test for differences in how trauma impacts on hallucinations and delusions. In other words, instead of analyzing the impact of trauma on each symptom separately (which would only indicate whether trauma is or is not significantly related to each symptom), this method tests whether the impact of trauma differs significantly for hallucinations or delusions. This approach is therefore preferable in the same sense that subgroup analyses in clinical trials should be conducted by testing proper interaction terms instead of analyzing subgroups separately ${ }^{10}$. Assessment of specificity of trauma for 
AVH or paranoia was done using the same analysis, now with presence of AVH or paranoia as outcomes. A (simplified) mathematical equation for this model can be found in box 1 .

\section{Abuse, neglect and intention-to-harm}

In order to investigate whether abuse or neglect have differential associations with psychosis, logistic regression analyses were applied. Presence of any PE was the dependent variable, and abuse (psychological, sexual or physical abuse) or neglect (emotional neglect) were dichotomous independent variables. As these analyses include only one outcome, mixedeffects analyses were not necessary. The difference of influence of abuse and neglect was thus established by post-hoc Wald test ${ }^{25}$. A similar analysis was performed to investigate whether CT with an intention-to-harm (psychological, sexual or physical abuse) or trauma without intent (death of a parent, sibling or close friend) have differential associations with psychosis.

Box 1 Mathematical equation for multilevel regression analysis with multiple outcomes, used to assess difference of influence of trauma on hallucinations versus delusions.

$\mathrm{y}^{\mathrm{A}}=\boldsymbol{\beta}_{0}+\beta_{1}\left(\right.$ dummy $\left.^{\mathrm{B}}\right)+\beta_{2}\left(\right.$ trauma $\left.^{\mathrm{C}}\right)+\beta_{3}\left(\right.$ confounder $\left.^{\mathrm{D}}\right)+\beta_{4}($ dummy)$)(\text { trauma })^{\mathrm{E}}+\beta_{5}$ (dummy)(confounder) $)^{\mathrm{F}}$

A Outcome is presence of any hallucinations $(0 / 1)$ or presence of any delusions $(0 / 1)$

B Dummy variable ( $0=$ hallucinations; $1=$ delusions) to distinguish between two outcome variables

C Each type of trauma (emotional neglect, psychological, physical or sexual abuse, growing up in foster care and the death of a parent, sibling or friend) was added simultaneously to the model (all six variables were used in the analysis)

$D$ The four confounders (depression, age, gender and cannabis use) were added to the model

E Interaction terms of each type of trauma and the dummy variable

F Interaction terms of each confounder and the dummy variable

$\beta_{4}$; Indicates statistical difference of influence of trauma on hallucinations versus delusions (if positive: stronger association with delusions; if negative: stronger association with hallucinations)

For each subject, two dichotomous outcomes were assessed, namely the presence of any hallucinations and the presence of any delusions. By using a random intercept term at the subject level, the influence of all the various childhood trauma types could be examined simultaneously on both outcomes. A dummy variable was added to the model to differentiate the two outcome variables. In addition, interaction terms between this dummy variable and the various childhood trauma variables were added to the model (i.e., to examine whether the influence of the childhood trauma variables differed for the two outcomes).

The coefficients of these interaction terms can therefore be interpreted as the differential association of each type of trauma on hallucinations versus delusions. Each of the six trauma variables (excluding the total trauma score) was added simultaneously to the model, in order to assess the specific association of each trauma type and psychosis (i.e the effect of one trauma type controlled for other trauma types). The influence of these potential confounders was allowed to differ across the two outcomes by including their respective interaction terms with the outcome dummy variable in the model. This analysis was conducted on the full sample with self-reported hallucinations and delusions as outcomes and complemented by a sensitivity analysis in the subsample with confirmed psychotic symptoms. 


\section{RESULTS}

\section{Prevalence rates}

The prevalence of at least one self-reported lifetime PE in both datasets combined was $17 \%$ (2359 of 13615 participants), or 18\% in NEMESIS-1 (1278 of 7076) and 16\% in NEMESIS-2 (1084 of 6646). The prevalence of at least one validated PE in NEMESIS-2 was 6\% (384 of 6357).

\section{Hallucinations and delusions}

Associations with traumatic experiences were significant across the entire range of PEs, except for death of a loved one, and these results were largely confirmed in the sensitivity analysis (Table 2). However, none of the trauma variables showed a significantly stronger association (at $p<0.001$ ) with delusions than with hallucinations, either in the full sample or in the sensitivity analysis (Table 3).

Moreover, emotional neglect and psychological abuse were more strongly associated with the co-occurrence of hallucinations and delusions than with isolated hallucinations (Table 4). In addition, a trend for a stronger association with co-occurring hallucinations and delusions compared with isolated hallucinations was also found for physical ( $\chi^{2} 3.65$, degrees of freedom[df] $1, p=0.0562)$ and sexual abuse $\left(\chi^{2} 5.25, d f 1, p=0.0220\right)$. Similarly, emotional neglect, psychological abuse, physical abuse and sexual abuse were all more strongly associated with a co-occurrence of hallucinations and delusions, compared with isolated delusions (Table 4). Growing up in foster care and death of a loved one were not associated more strongly with co-occurrence of hallucinations and delusions compared

with isolated hallucinations $\left(\chi_{\text {foster }}^{2} 0.24, \mathrm{df} 1, \mathrm{p}=0.626 ; \chi_{\text {death }}^{2} 0.85, \mathrm{df} 1, \mathrm{p}=0.357\right.$ ) or isolated delusions ( $\chi_{\text {foster }}^{2} 0.10$, df $1, p=0.754 ; \chi_{\text {death }}^{2} 0.42$, df $\left.1, p=0.518\right)$. 
Table 2 NEMESIS- 1 and -2 baseline measurement. Effect sizes of each type of trauma and hallucinations and delusions, self-reported and validated by reinterview (sensitivity analysis)

\begin{tabular}{|c|c|c|c|c|c|c|c|c|}
\hline & $\begin{array}{l}\text { Hallucinations } \\
\text { (self-report) }\end{array}$ & & $\begin{array}{l}\text { Hallucinations } \\
\text { (interview }{ }^{A} \text { ) }\end{array}$ & & $\begin{array}{l}\text { Delusions } \\
\text { (self-report) }\end{array}$ & & $\begin{array}{l}\text { Delusions } \\
\text { (interview) }\end{array}$ & \\
\hline $\begin{array}{l}\text { Continuous trauma } \\
\text { scales (standardized) }\end{array}$ & $\begin{array}{l}\mathrm{OR}^{\mathrm{B}} \\
\left(95 \% \mathrm{Cl}^{\mathrm{C}}\right)\end{array}$ & $\mathrm{p}$ & $\begin{array}{l}\text { OR } \\
(95 \% \mathrm{Cl})\end{array}$ & $p$ & $\begin{array}{l}\text { OR } \\
(95 \% \mathrm{Cl})\end{array}$ & $\mathrm{p}$ & $\begin{array}{l}\text { OR } \\
(95 \% \mathrm{Cl})\end{array}$ & $\mathrm{p}$ \\
\hline Total trauma ${ }^{\mathrm{D}}$ & $1.39(1.32-1.46)$ & 0.000 & $1.36(1.23-1.50)$ & 0.000 & $1.35(1.29-1.41)$ & 0.000 & $1.43(1.30-1.57)$ & 0.000 \\
\hline Emotional neglect ${ }^{\mathrm{E}}$ & $1.30(1.23-1.37)$ & 0.000 & $1.32(1.19-1.47)$ & 0.000 & $1.31(1.25-1.37)$ & 0.000 & $1.40(1.27-1.54)$ & 0.000 \\
\hline Psychological abuse ${ }^{\mathrm{E}}$ & $1.34(1.27-1.41)$ & 0.000 & $1.28(1.15-1.43)$ & 0.000 & $1.28(1.23)$ & 0.000 & $1.34(1.22-1.49)$ & 0.000 \\
\hline Physical abuse ${ }^{\mathrm{E}}$ & $1.25(1.20-1.31)$ & 0.000 & $1.27(1.15-1.39)$ & 0.000 & $1.20(1.15-1.25)$ & 0.000 & $1.26(1.15-1.38)$ & 0.000 \\
\hline Sexual abuse $\mathrm{E}^{\mathrm{E}}$ & $1.22(1.17-1.28)$ & 0.000 & $1.22(1.11-1.33)$ & 0.000 & $1.16(1.12-1.21)$ & 0.000 & $1.20(1.10-1.31)$ & 0.000 \\
\hline $\begin{array}{l}\text { Growing up in foster } \\
\text { care }^{F}\end{array}$ & $2.11(1.45-3.07)$ & 0.000 & $1.80(0.70-4.64)$ & 0.221 & $1.80(1.29-2.52)$ & 0.001 & $2.12(0.87-5.15)$ & 0.097 \\
\hline $\begin{array}{l}\text { Death of parent, } \\
\text { sibling or friend }{ }^{F}\end{array}$ & $1.00(0.83-1.19)$ & 0.976 & $0.77(0.47-1.28)$ & 0.316 & $1.21(1.05-1.39)$ & 0.008 & $0.98(0.65-1.48)$ & 0.925 \\
\hline
\end{tabular}

Note: All effect sizes obtained by separate regression analyses, a priori confounders added are depression, cannabis use, age and gender. Only the effect sizes in bold are considered significant, set at the more stringent level of 0.001 .

A Interview-based validation of psychotic symptoms (NEMESIS-2 subsample); sensitivity analysis

${ }^{B}$ Odds Ratio

c $95 \%$ confidence interval

${ }^{D}$ Overall trauma score (combination of emotional neglect, psychological abuse, physical abuse and sexual abuse) range 0 to 20; thus Odds Ratios (OR) linear trend

E Trauma score range 0 (never) to 5 (very often); thus OR's linear trend

F Binary trauma variable 
Table 3 NEMESIS- 1 and -2 baseline assessment. Associations (at $p<0.001$ ) of all trauma types and hallucinations versus delusions; self-reported and validated by reinterview sensitivity analysis).

\begin{tabular}{|c|c|c|c|c|}
\hline & \multicolumn{2}{|c|}{$\begin{array}{l}\text { Hallucinations vs Delusion } \\
\text { (self-report) }\end{array}$} & \multicolumn{2}{|c|}{$\begin{array}{l}\text { Hallucinations vs Delusions } \\
\text { (interview }{ }^{A} \text { ) }\end{array}$} \\
\hline & $\mathrm{OR}^{\mathrm{B}}\left(\mathrm{Cl}^{\mathrm{C}}\right)$ & p-value & $\mathrm{OR}^{\mathrm{B}}(\mathrm{Cl})$ & p-value \\
\hline Emotional neglect & $1.13(1.01-1.26)$ & 0.028 & $1.12(0.85-1.48)$ & 0.426 \\
\hline Psychological abuse & $0.93(0.84-1.05)$ & 0.242 & $1.08(0.79-1.48)$ & 0.612 \\
\hline Physical abuse & $0.95(0.87-1.04)$ & 0.304 & $0.91(0.71-1.17)$ & 0.483 \\
\hline Sexual abuse & $0.93(0.86-1.00)$ & 0.064 & $0.93(0.76-1.14)$ & 0.489 \\
\hline Growing up in foster care & $0.78(0.42-1.45)$ & 0.432 & $1.07(0.14-8.25)$ & 0.943 \\
\hline $\begin{array}{l}\text { Death of a parent, sibling or } \\
\text { close friend }\end{array}$ & $1.36(1.03-1.79)$ & 0.027 & $1.65(0.70-3.90)$ & 0.250 \\
\hline
\end{tabular}

Note: Each coefficient expresses the differential influence of trauma on hallucinations versus delusions. A priori confounders added are depression, cannabis use, age and gender. All trauma variables were added simultaneously to the model. Significance is set at more stringent level of 0.001 .

A Interview-based validation of psychotic symptoms (NEMESIS-2 subsample); sensitivity analysis

B $O R>1$ indicates stronger association with delusions, $O R<1$ indicates stronger association with hallucinations

C $95 \%$ confidence interval 


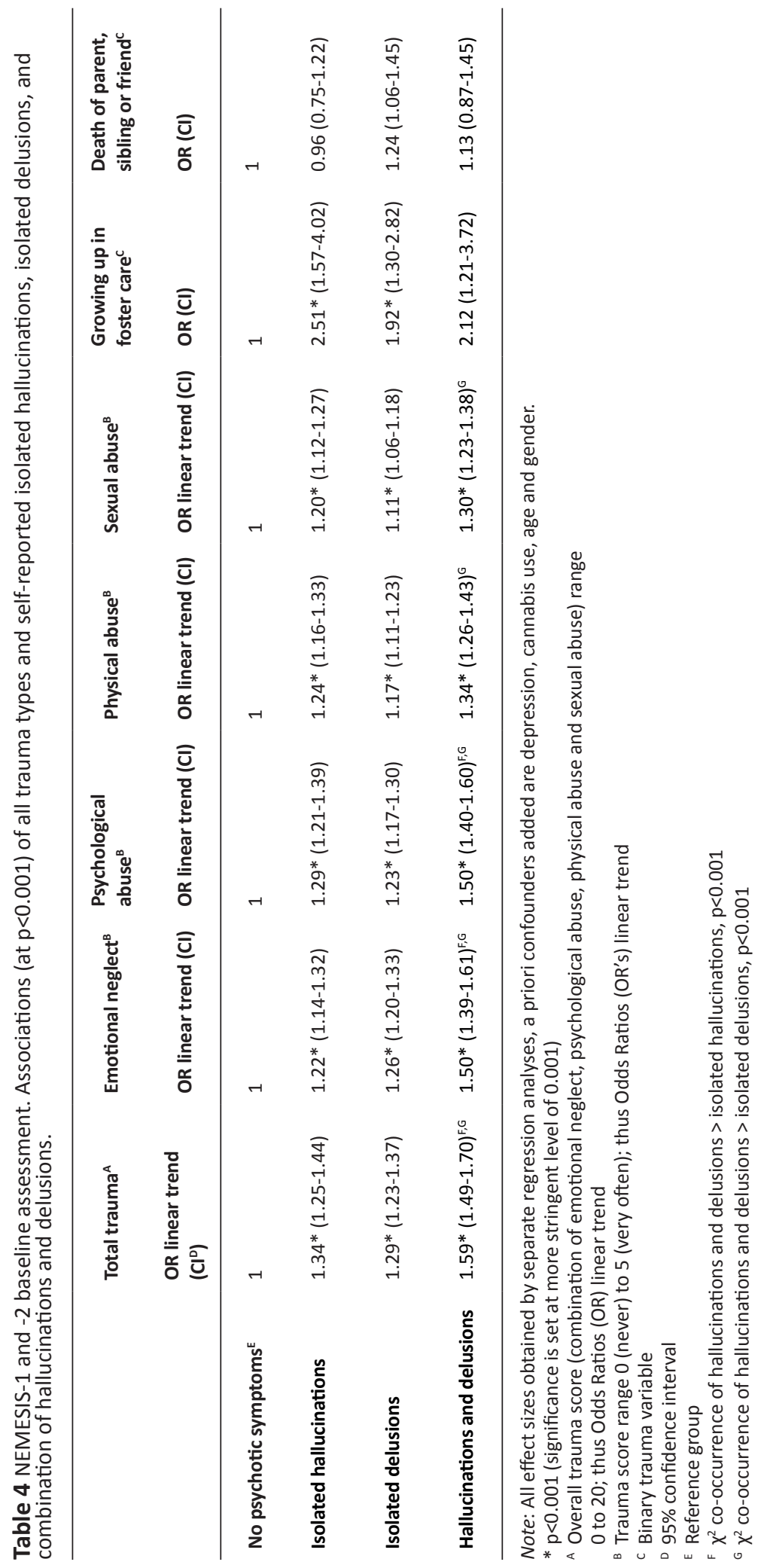




\section{AVH and paranoia}

Results from the separate regression analyses are shown in Table 5. None of trauma variables had a specific or significantly stronger association with AVH or with paranoia, in either the full sample or in the sensitivity analysis (Table 6).

Table 5 NEMESIS- 1 and -2 baseline measurement. Effect sizes of each type of trauma and hallucinations and delusions, self-reported and validated by reinterview (sensitivity analysis)

\begin{tabular}{|c|c|c|c|c|c|c|c|c|}
\hline & $\begin{array}{l}\text { AVH }^{A} \\
\text { (self-report) }\end{array}$ & & $\begin{array}{l}\text { AVH } \\
\text { (interview }{ }^{\mathrm{B}} \text { ) }\end{array}$ & & $\begin{array}{l}\text { Paranoia } \\
\text { (self-report) }\end{array}$ & & $\begin{array}{l}\text { Paranoia } \\
\text { (interview) }\end{array}$ & \\
\hline $\begin{array}{l}\text { Continuous } \\
\text { trauma scales } \\
\text { (standardized) }\end{array}$ & $\begin{array}{l}\mathrm{OR}^{\mathrm{C}} \\
\left(95 \% \mathrm{Cl}^{\mathrm{D}}\right)\end{array}$ & p & $\begin{array}{l}\text { OR } \\
(95 \% \mathrm{Cl})\end{array}$ & p & $\begin{array}{l}\text { OR } \\
(95 \% \mathrm{Cl})\end{array}$ & $\mathbf{p}$ & $\begin{array}{l}\text { OR } \\
(95 \% \mathrm{Cl})\end{array}$ & $\mathbf{p}$ \\
\hline Total trauma ${ }^{\mathrm{E}}$ & $1.42(1.30-1.55)$ & 0.000 & $1.30(1.10-1.54)$ & 0.002 & $1.34(1.28-1.41)$ & 0.000 & $1.46(1.30-1.65)$ & 0.000 \\
\hline Emotional neglect ${ }^{F}$ & $1.38(1.25-1.53)$ & 0.000 & $1.26(1.05-1.51)$ & 0.012 & $1.29(1.22-1.35)$ & 0.000 & $1.37(1.21-1.56)$ & 0.000 \\
\hline Psychological abuse ${ }^{F}$ & $1.34(1.22-1.48)$ & 0.000 & $1.21(1.00-0.47)$ & 0.052 & $1.29(1.23-1.35)$ & 0.000 & $1.38(1.21-1.58)$ & 0.000 \\
\hline Physical abuse ${ }^{F}$ & $1.25(1.16-1.36)$ & 0.000 & $1.24(1.05-1.45)$ & 0.009 & $1.20(1.15-1.25)$ & 0.000 & $1.27(1.13-1.43)$ & 0.000 \\
\hline Sexual abuse ${ }^{\mathrm{F}}$ & $1.28(1.20-1.38)$ & 0.000 & $1.26(1.10-1.44)$ & 0.001 & $1.17(1.12-1.23)$ & 0.000 & $1.27(1.14-1.42)$ & 0.000 \\
\hline $\begin{array}{l}\text { Growing up in } \\
\text { foster care }\end{array}$ & $2.44(1.21-4.92)$ & 0.013 & $2.31(0.54-9.84)$ & 0.258 & $1.74(1.19-2.54)$ & 0.004 & $3.27(1.25-8.52)$ & 0.015 \\
\hline $\begin{array}{l}\text { Death of parent, } \\
\text { sibling or friend }\end{array}$ & $1.47(1.04-2.08)$ & 0.029 & $0.61(0.24-1.54)$ & 0.295 & $1.20(1.02-1.40)$ & 0.028 & $1.01(0.57-1.80)$ & 0.959 \\
\hline
\end{tabular}

Note: All effect sizes obtained by separate regression analyses, a priori confounders added are depression, cannabis use, age and gender. Only the effect sizes in bold are considered significant, set at the more stringent level of 0.001 .

${ }^{\text {A }}$ Auditory-verbal hallucinations

B Interview-based validation of psychotic symptoms (NEMESIS-2 subsample); sensitivity analysis

C Odds Ratio

D 95\% confidence interval

E Overall trauma score (combination of emotional neglect, psychological abuse, physical abuse and sexual abuse) range 0 to 20; thus Odds Ratios (OR) linear trend

F Trauma score range 0 (never) to 5 (very often); thus OR's linear trend

G Binary trauma variable 
Table 6 NEMESIS- 1 and -2 baseline assessment. Associations (at $p<0.001$ ) of all trauma types and hallucinations versus delusions; self-reported and validated by reinterview sensitivity analysis).

\begin{tabular}{lllll}
\hline & \multicolumn{2}{l}{ AVH $^{\mathrm{A}}$ vs Paranoia (self-report) } & \multicolumn{2}{l}{ AVH vs Paranoia (interview ${ }^{\mathrm{B}}$ ) } \\
& $\mathrm{OR}^{\mathrm{C}}\left(\mathrm{Cl}^{\mathrm{D}}\right)$ & $\mathrm{p}$-value & OR $(\mathrm{Cl})$ & p-value \\
\hline Emotional neglect & $0.98(0.82-1.16)$ & 0.804 & $1.16(0.79-1.70)$ & 0.445 \\
Psychological abuse & $1.08(0.90-1.30)$ & 0.390 & $1.23(0.80-1.88)$ & 0.335 \\
Physical abuse & $0.97(0.84-1.12)$ & 0.662 & $0.84(0.61-1.19)$ & 0.333 \\
Sexual abuse & $0.91(0.83-1.02)$ & 0.096 & $1.04(0.81-1.34)$ & 0.770 \\
Growing up in foster care & $0.89(0.34-2.34)$ & 0.810 & $3.06(0.13-72.24)$ & 0.487 \\
$\begin{array}{l}\text { Death of a parent, sibling } \\
\text { or close friend }\end{array}$ & $0.84(0.54-1.28)$ & 0.419 & $1.65(0.43-6.30)$ & 0.465 \\
\hline
\end{tabular}

Note: Each coefficient expresses the differential influence of trauma on auditory-verbal hallucinations versus paranoia. A priori confounders added are depression, cannabis use, age and gender. All trauma variables were added simultaneously to the model. Significance is set at more stringent level of 0.001 .

A Auditory-Verbal hallucinations

${ }^{B}$ Interview-based validation of psychotic symptoms (NEMESIS-2 subsample); sensitivity analysis

c $\mathrm{OR}>1$ indicates stronger association with paranoia, $\mathrm{OR}<1$ indicates stronger association with AVH

D $95 \%$ confidence interval

\section{Abuse, neglect and intention-to-harm}

In the full sample, abuse (OR 2.12, $\mathrm{Cl} 1.92-2.35, \mathrm{p}<0.001)$ and neglect (OR 1.96, $\mathrm{Cl} 1.76-$ 2.17, $p<0.001$ ) were both associated with self-reported PEs. Post-hoc analysis showed that the effect of one was not larger than the other $\left(\chi^{2} 2.77, \mathrm{df} 1, \mathrm{p}=0.0961\right)$. Similarly, in the sensitivity analysis both abuse (OR 2.19, $\mathrm{Cl} 1.74-2.75, \mathrm{p}<0.001$ ) and neglect (OR 2.49, $\mathrm{Cl} 1.94-3.20, p<0.001)$ were associated with psychosis, however of equivalent effect size $\left(\chi^{2} 0.21\right.$, df $\left.1, p=0.646\right)$.

Individuals reporting CT with intention-to-harm had an increased likelihood of reporting self-reported PEs (OR 2.12, Cl 1.92-2.35, p<0.001). This was also true for individuals reporting CT without intention-to-harm, although at much lower effect size and at trendlevel significance (OR 1.14, $\mathrm{Cl} 1.00-1.29, \mathrm{p}=0.047$ ). Post-hoc analysis showed that the association between trauma with intention-to-harm and psychosis was significantly stronger $\left(\chi^{2} 58.62\right.$, df $\left.1, p<0.001\right)$.

Comparable results were found in the sensitivity analysis, in that trauma with intention-toharm was associated with validated PEs (OR 2.19, $\mathrm{Cl} 1.74-2.75, \mathrm{p}<0.001)$, whereas trauma without intention-to-harm was not (OR 0.88, $\mathrm{Cl} 0.61-1.28, \mathrm{p}=0.512$ ). Post-estimation analysis confirmed that the association between trauma with intention-to-harm and psychosis was significantly stronger $\left(\chi^{2} 16.91, \mathrm{df} 1, \mathrm{p}<0.001\right)$. 


\section{DISCUSSION}

This study confirms earlier findings of associations between CT on the one hand, and hallucinations and delusions on the other ${ }^{2,5-7}$. Most types of childhood trauma were associated with an increased probability of co-occurrence of hallucinations and delusions, rather than their isolated occurrence, in agreement with previous findings ${ }^{4}$.

No evidence was found for specificity of any of the trauma variables for any of the psychotic symptoms, except for trauma with or without 'intention-to-harm'. Trauma with 'intention-to-harm' showed a stronger association with PE, compared to trauma without 'intention-to-harm'.

\section{Social defeat, intention-to-harm experiences and psychosis}

The finding that the experience of 'intention-to-harm' is a key factor in the association between $\mathrm{CT}$ and psychosis may fit with the 'social defeat' hypothesis of psychosis's, which postulates that several environmental risk factors for psychosis, including $\mathrm{CT}$, may increase feelings of outsider status, which in turn is associated with psychosis ${ }^{26}$. However, one could argue that the loss of a parent may also result in an outsider status in children, as afterwards peers may have difficulty including them in social activities. As this type of trauma showed a weaker association with psychosis in the present study, this would suggest that not feelings of social defeat itself, but perceived social threat, especially present in bullying or other types of abuse, could be the key risk factor for psychosis, in agreement with previous work ${ }^{9}$.

\section{Co-occurrence of hallucinations and delusions}

The present study found evidence for an increased probability of co-occurrence of hallucinations and delusions associated with CT. Previous work has reported similar results, including an increased risk of this co-occurrence associated with other known environmental risk factors for psychosis, such as cannabis use and urbanicity ${ }^{4,27}$, indicating higher levels of etiological loading in individuals reporting both symptoms. Furthermore, co-occurrence of these symptoms has been shown to be associated with an increase in help-seeking and greater severity of symptoms ${ }^{4}$. Therefore, the data suggest that CT increases risk for intensification of the psychotic process, causing buildup from simple to more complex psychotic states that increase the risk for help-seeking and, finally, clinical decompensation.

\section{Specificity in the association between childhood trauma and psychosis}

Other than differences in associations with psychosis when assessing the presence of 'intention-to-harm', no other evidence of specificity, in terms of trauma variables or symptoms, was found. Even though the theory that childhood abuse leads to source 
monitoring deficits, resulting in hallucinations, may be appealing ${ }^{7}$, it does not exclude the possibility that negative views about the world and others may arise, which have been suggested to fuel delusions ${ }^{28}$.

Furthermore, as the present findings indicate that all trauma variables, including sexual abuse and growing up in foster care, did not show statistically dissimilar associations with paranoia and $\mathrm{AVH}$, assumptions about specific psychological processes following these trauma variables may not apply?.

\section{Statistical methods}

The present results underscore the importance of using mixed-effects logistic regression models for assessing specificity in effects of trauma on symptoms. For example, in this sample, physical abuse showed a significant association with paranoia, and no significant association with $\mathrm{AVH}$. By using mixed-effects logistic regression it was revealed that these effect sizes were not statistically different, in contrast to what previous work had suggested ${ }^{7}$. Similarly, by statistically comparing the effect sizes of abuse and neglect it was shown that neither abuse nor neglect showed a stronger association with psychosis, again not confirming previous interpretations ${ }^{5,6}$.

\section{Strengths and weaknesses}

To the best of our knowledge, this is the first study including such a wide array of aspects regarding the association between $\mathrm{CT}$ and psychosis, using a large representative sample of the Dutch population. In addition, the quality of the psychological assessments was high, as self-reported PE were re-assessed by clinical interview (by an experienced rater with clinical experience), thus decreasing the possibility of false-positives. An important limitation of this study lies in its cross-sectional nature, with lifetime assessment of symptoms (precluding inferences on chronological order of trauma and symptoms), abolishing any claims of causality. Furthermore, as CT was assessed using retrospective reports, recall bias may have influenced these results, although other studies have found that recall of childhood trauma is reliable, even in individuals with presence of psychosis ${ }^{29}$.

\section{Funding}

This work was supported by the Ministry of Health, Welfare and Sports (grant number 310253); with supplement support from the Netherlands Organization for Health Research and Development (ZonMw) and the Genetic Risk and Outcome of Psychosis (GROUP) investigators. Supported by the European Community's Seventh Framework Program under grant agreement No.HEALTH-F2-2009-241909(Project EU-GEI). 


\section{REFERENCES}

1. Varese F, Smeets F, Drukker M, et al. Childhood adversities increase the risk of psychosis: a meta-analysis of patient-control, prospective- and cross-sectional cohort studies. Schizophr Bull 2012;38(4):661-671.

2. Read J, Agar K, Argyle N, Aderhold V. Sexual and physical abuse during childhood and adulthood as predictors of hallucinations, delusions and thought disorder. Psychol

Psychother-T 2003;76:1-22.

3. Kapur S. Psychosis as a state of aberrant salience: a framework linking biology, phenomenology, and pharmacology in schizophrenia. Am J Psychiat 2003;160:13-23.

4. Smeets F, Lataster T, Dominguez MG, Hommes J, Lieb R, Wittchen HU, van Os J. Evidence That Onset of Psychosis in the Population Reflects Early Hallucinatory Experiences That Through Environmental Risks and Affective Dysregulation Become Complicated by Delusions. Schizophr Bull 2012;38(3):531-542.

5. Heins $M$, Simons $C$, Lataster $T$, et al. Childhood trauma and psychosis:

A case-control and case-sibling comparison across different levels of genetic liability, psychopathology, and type of trauma. Am J Psychiat 2011;168(12):1286-1294.

6. Shevlin M, Dorahy M, Adamson G. Childhood traumas and hallucinations: An analysis of the National Comorbidity Survey. J Psychiat Res 2007;41:222-228.

7. Bentall R, Wickham S, Shevlin M, Varese F. Do specific early-life adversities lead to specific symptoms of psychosis? A study from the 2007 the adult psychiatric morbidity survey. Schizophr Bull

2012;38(4):734-740.

8. Selten JP, Cantor-Graae E. Social defeat: risk factor for schizophrenia? B J Psychiat 2005;187:101-102.

9. Arseneault $L$, Cannon $M$, Fisher $\mathrm{H}$, Polanczyk G, Moffitt TE, Caspi A. Childhood trauma and children's emerging psychotic symptoms: a genetically sensitive longitudinal cohort study. Am J Psychiat 2011;168:65-72.

10. Pocock SJ, Assmann SE, Enos LE, Kasten LE. Subgroup analysis, covariate adjustment and baseline comparisons in clinical trial reporting: current practice and problems. Stat Med 2002;21(19):2917-2930.

11. Smeets RMW, Dingemans PMAJ. The Composite International Diagnostic Interview (CIDI) version 1.1 (Dutch version).Geneva, Switserland: World Health Organization, 1993.

12. Alonso J, Angermeyer M, Bernert $S$, et al. Sampling and methods of the European Study of the Epidemiology of Mental Disorders (ESEMeD) project. Acta Psychiat Scand 2004;109(Suppl. 420):8-20.

13. Bijl RV, van Zessen G, Ravelli A, de Rijk $C$, Langendoen Y. The Netherlands Mental Health Survey and Incidence Study (NEMESIS): Objectives and design Soc Psych Psych Epidem 1998;33:581-586.

14. De Graaf $R$, ten Have $M$, van Dorsselaer S. The Netherlands Mental Health Survey and Incidence study-2 (NEMESIS-2): design and methods. Int J Meth Psychiat Res 2010;19(3):125-141.

15. Andrew G, Peters $L$. The psychometric properties of the Composite 
International Diagnostic Interview.

Soc Psych Psych Epidem

1998;33:80-88.

16. van Nierop M, Van Os J, Gunther N, et al. Phenotypically continuous with clinical psychosis, discontinuous in need for care: Evidence for an extended psychosis phenotype. Schizophr Bull 2012;38(2):231-238.

17. Spitzer RL, Williams JB, Gibbon M, First MB. The Structured Clinical Interview for DSM-III-R (SCID). I: History, rationale, and description. Arch Gen Psychiat 1992;49(8):624629.

18. Janssen I, Krabbendam L, Bak M, Hanssen M, Vollebergh W, De Graaf R, Van Os J. Childhood abuse as a risk factor for psychotic experiences. Acta Psychiat Scand 2004;109:38-45.

19. Krabbendam L, Myin-Germeys I, de Graaf R, Vollebergh W, Nolen WA, ledam J, van Os J. Dimensions of depression, mania and psychosis in the general population. Psychol Med 2004;34:1177-1186.

20. Tijssen MJA, van Os J, Wittchen HU, Lieb R, Beesdo K, Mengelers R, Wichers M. Prediction of transition from common adolescent bipolar experiences to bipolar disorder: a 10 year study. $B$ J Psychiat 2010;196:102-108.

21. Van Os J, Bak M, Hanssen M, Bijl RV, De Graaf R, Verdoux H. Cannabis use and psychosis: a longitudinal population-based study. Am J Epidem 2002;156:319-327.

22. Statacorp. Stata Statistical Software:

Release 11. College Station, TX: StataCorp LP. 2009.

23. Wigman JTW, van Nierop $M$, Vollebergh W, Lieb R, Beesdo-Baum K,
Wittchen HU, Van Os J. Evidence that psychotic symptoms are prevalent in disorders of anxiety and depression, impacting on illness onset, risk, and severity - implications for diagnosis and ultra-high risk research. Schizophr Bull 2012;38(2):247-257.

24. Moore THM, Zammit $S$, LingfordHughes A, Barnes TRE, Jones PB, Burke $M$, Lewis $G$. Cannabis use and risk of psychotic or affective mental health outcomes: a systematic review. The Lancet 2007;370(9584):319-328.

25. Clayton D, Hills M. Statiatical Models in Epidemiology. Oxford: OUP; 1993.

26. Van Winkel R, Van Nierop M, MyinGermeys I, van Os J. Childhood Trauma as a Cause of Psychosis: Linking Genes, Psychology, and Biology. Can J Psychiat 2013;58(1):44-51.

27. Smeets $F$, Lataster $T$, van Winkel $R$, De Graaf R, Ten Have M, Van Os J. Testing the hypothesis that psychotic illness begins when subthreshold hallucinations combine with delusional ideation. Acta Psychiat Scand 2012; DOI: 10.1111/j.1600-0447.2012.01888.x.

28. Read J, van Os J, Morrison AP, Ross CA. Childhood trauma, psychosis and schizophrenia: a literature review with theoretical and clinical implications. Acta Psychiat Scand 2005;112:330-350.

29. Fisher HL, Craig TK, Fearon $P$, et al. Reliability and comparability of psychosis patients' retrospective reports of childhood abuse. Schizophr Bull 2011;37(3):546-553. 


\title{
CHAPTER 5
}

Childhood Trauma as a Cause of Psychosis: Linking Genes, Psychology and Biology

\author{
Ruud van Winkel ${ }^{A B}$ \\ Martine van Nierop ${ }^{A}$ \\ Inez Myin-Germeys ${ }^{A}$ \\ Jim van $\mathrm{Os}^{\mathrm{AC}}$
}

A Dept. of Psychiatry and Psychology, School for Mental Health and Neuroscience, EURON, Maastricht University Medical Centre, PO Box 616 (Vijv1), 6200 MD Maastricht, The Netherlands

B University Psychiatric Center Katholieke Universiteit Leuven, campus Kortenberg, Leuvensesteenweg, Kortenberg, Belgium

c King's College London, King's Health Partners, Department of Psychosis Studies Institute of Psychiatry, London, UK

Canadian Journal of Psychiatry 2013; 58(1): $44-51$ 


\section{ABSTRACT}

Recent studies have provided robust evidence for an association between childhood trauma and psychosis. Meta-analyses have quantified the association, pointing to odds ratios in the order of around 3 , and prospective studies have shown that reverse causation is unlikely to explain the association. However, more work is needed to address the possibility of gene-environment correlation, that is, whether genetic risk for psychosis predicts exposure to childhood trauma. Nevertheless, multiple studies have convincingly shown that the association between childhood trauma and psychosis remains strong and significant when controlling for genetic risk, in agreement with a possible causal association. In addition, several studies have shown plausible psychological and neurobiological mechanisms linking adverse experiences to psychosis, including induction of social defeat and reduced self-value, sensitization of the mesolimbic dopamine system, changes in the stress and immune system and concomitant changes in stress-related brain structures such as the hippocampus and the amygdala, findings that should be integrated, however, in more complex models of vulnerability. It is currently unclear whether genetic vulnerability plays a role in conferring the mental consequences of adversity, and which genes are likely to be involved. The current, limited evidence points to genes that are not specifically involved in psychosis but more generally in regulating mood (serotonin transporter gene), neuroplasticity (brain-derived neurotrophic factor) and the stressresponse system (FKBP5), in line with a general effect of childhood trauma on a range of mental disorders rather, than suggesting specificity for psychosis. 


\section{INTRODUCTION}

Several factors in the social environment have been associated with an increased risk for psychotic disorder ${ }^{1}$. Of these, early life adversity, or childhood trauma, is one of the most extensively studied social factors associated with the development of psychosis ${ }^{2}$. Childhood trauma is a general term that refers to a range of adverse experiences before the age of 16 years, including sexual, physical and emotional abuse, and neglect. An early review on this topic generated controversy, claiming that childhood trauma is a causal factor for psychosis and schizophrenia ${ }^{3}$. However, other reviews concluded that this was premature given the methodological limitations of the studies on which this conclusion was based ${ }^{2,4}$. This review provides an update on the studies that have examined the association between childhood trauma and psychosis, including two recently published meta-analyses. In addition, we will identify remaining issues that need to be resolved before claims of causality can be substantiated. Third, we will provide an overview of psychological and biological mechanisms that could plausibly tie childhood trauma to risk of psychosis.

\section{Evidence for association}

Since the reviews of Read ${ }^{3}$, Morgan and Fisher ${ }^{4}$, and Bendall ${ }^{2}$, numerous novel, methodologically rigorous studies have been published ${ }^{5-11}$. For example, in a prospective study investigating the association between childhood trauma and psychotic disorder, a large cohort of adolescents and adults ( $N=2759$, age range $15-58$ years) who were sexually abused before the age of 16 years were compared with age and gender matched nonabused controls $(\mathrm{N}=2677)$ from the general population ${ }^{6}$. Psychiatric case registers were used to assess the history of mental health problems. Sexual abuse was assessed using police records, hence excluding the possibility of recall bias. This study found a two-fold increased risk for a diagnosis of any psychotic disorder and a 2.6-fold increased risk for schizophrenia ${ }^{6}$.

Other investigators have studied the broader concept of psychotic experiences as opposed to the narrower concept of a clinical diagnosis of psychotic disorder, 9 . For example, Arseneault and colleagues followed 2232 twin children and their families ${ }^{7}$. Participants (twin children and their mothers) were interviewed at home when the children were 7, 10 and 12 years old. Childhood adversity (maltreatment by an adult, peer victimization, and accidents) was assessed by interviewing the children and the mothers separately. Psychotic experiences were assessed by interviewing the children and mothers at the last follow-up interview. The study found that maltreatment by an adult and peer victimization (intention to harm experiences) were associated with a significantly increased risk for the development of psychotic experiences (OR 3.3,95\% $\mathrm{Cl}$ 2.25-4.76), even after adjusting for individual and socioeconomic factors, other child psychopathology and genetic vulnerability for psychosis ${ }^{7}$.

Two recent extensive meta-analyses have systematically quantified the association between childhood trauma and psychosis. Varese and colleagues ${ }^{12}$ included 36 studies (18 
case-control, 10 [quasi] prospective and 8 population-based cross-sectional studies) with over 80.000 subjects combined. Psychosis was defined as having either (sub-clinical) psychotic experiences, or psychotic disorder. Childhood trauma was defined as sexual, physical, or emotional abuse, neglect, bullying, and parental death, before the age of 18 . The overall Odds Ratio (OR) of psychosis after childhood trauma was 2.8, with comparable OR's for each of the three types of studies: case-control studies 2.7 (95\% Cl 1.90-3.88); prospective studies 2.8 (95\% Cl 2.17-3.47); and population-based studies $3.0(95 \% \mathrm{Cl}$ 2.12-4.20). The OR's for specific types of trauma were also reported: bullying (OR 2.4), emotional abuse (OR 3.4), neglect (OR 2.9), physical abuse (OR 3.0), and sexual abuse (OR 2.4). The OR of psychosis after parental death was 1.7 , but this result was not significant. The authors were unable to include a synthesis of data on a possible dose-response relationship between trauma and psychosis, because of heterogeneous definitions applied in the primary studies. However, 9 out of 10 studies that examined this issue were in support of a dose-response relationship ${ }^{12}$.

In another recent meta-analysis ${ }^{13}$, the specificity of the association between childhood trauma and psychosis was investigated. The authors included studies investigating childhood trauma in schizophrenia patients versus non-psychiatric controls $(\mathrm{N}=7)$, affective psychosis $(\mathrm{N}=8)$, anxiety disorders $(\mathrm{N}=7)$, depressive disorders $(\mathrm{N}=7)$, dissociative disorders and posttraumatic stress disorder $(\mathrm{N}=4)$, other psychosis $(\mathrm{N}=3)$, and compared with personality disorders $(\mathrm{N}=3)$. When comparing the occurrence of childhood trauma in schizophrenia patients with non-psychiatric controls, an OR of $3.6(p<0.001)$ was found. As expected, given extensive literature on the effect of childhood trauma across a range of mental disorder outcomes, the risk of developing any of the other major psychiatric disorders following childhood trauma was comparable, with the exception of anxiety disorders, pointing to a non-specific association with psychotic disorder ${ }^{13}$. This is remarkably similar to reports of the non-specificity of family history across the range of mental disorders ${ }^{14,15}$.

\section{Causal association?}

Given that the robustness of the association now has been clearly established, it is important to consider alternative explanations before any claim of causality can be substantiated. These concern reverse causation and gene-environment correlation ${ }^{16}$.

In this regard, reverse causation would be the theory that early symptoms of psychosis lead to an increased risk of exposure to childhood adversity rather than the other way around (Figure $1 \mathrm{~A}$ and $\mathrm{B}$ ). Is this a plausible explanation for the association between childhood trauma and psychosis? The timing of the exposure and the first occurrence of symptoms are crucially important in this regard. In agreement with this temporal order, prospective studies have shown that childhood adverse experiences are most likely to occur before the onset of psychosis ${ }^{17}$. Cutajar and colleagues demonstrated that childhood adverse experiences predicted clinical psychosis later in life, by linking police records of reports of childhood sexual abuse to psychiatric case registers ${ }^{6}$. The mean age at the 
allegation of abuse was 10 years and the first youth to use psychiatric services for psychosis did this when they were 14 years old. However, these findings do not completely exclude the possibility of reverse causation, because attenuated psychotic experiences could have been present before the onset of sexual abuse. Similarly, though Arseneault and colleagues ${ }^{7}$ assessed childhood trauma at ages 7, 10 and 12 years, they only assessed psychotic experiences at age 12 years. In another prospective study, investigating the association between bullying (at age 8 or 10 years) and subsequent psychotic experiences (at age 13 years), the same issue remains: psychotic experiences were not assessed prior to onset of bullying ${ }^{18}$.

A second, non-mutually exclusive explanation for the association between childhood trauma and psychosis is gene-environment correlation. In case of gene-environment correlation, a genetic predisposition leads both to the later expression of psychosis as well as an increased likelihood of exposure to early life adversity. In this case, trauma has no additional impact on the occurrence of psychotic symptoms (Figure $1 \mathrm{C})^{16}$. It is not unlikely that individuals at increased genetic risk for psychosis may be more vulnerable to victimization because of traits associated with psychosis, such as cognitive impairments and impaired social functioning. To our knowledge, no studies have specifically investigated this explanation. One reason for this is the difficulty of defining genetic risk for psychosis. A study of patients with psychosis, their unaffected siblings and healthy controls reported associations between childhood adversity and psychosis across all levels of familial liability ${ }^{5}$. In addition, the unaffected siblings were more likely to have experienced childhood trauma than the healthy controls, which could be indicative of gene-environment correlation ${ }^{5}$. As many of the relevant adverse experiences pertain to the family environment, the increased prevalence of adversity in siblings in this study may therefore merely represent familial clustering of traumatic experiences per se (i.e. unrelated to genetic risk for psychosis) rather than true gene-environment correlation.

Other studies have not investigated gene-environment correlation, but whether childhood trauma may still have an effect when controlling for genetic risk. All of these studies reported strong and significant associations between childhood adversity and psychosis, even when controlling for genetic risk ${ }^{7,19,20}$. These studies thus suggest that, even when assuming that genetic risk for psychosis increases the likelihood of exposure to childhood trauma, the actual exposure in itself further increases the risk for psychosis. The conclusion would be that the association is not merely be confounding or gene-environment correlation, but rather (partial) genetic mediation of environmental effects (Figure 1D). This would support an etiological role for the exposure to trauma on the development of psychosis ${ }^{3}$. 
Figure 1 Etiological models explaining the association between childhood trauma and psychosis

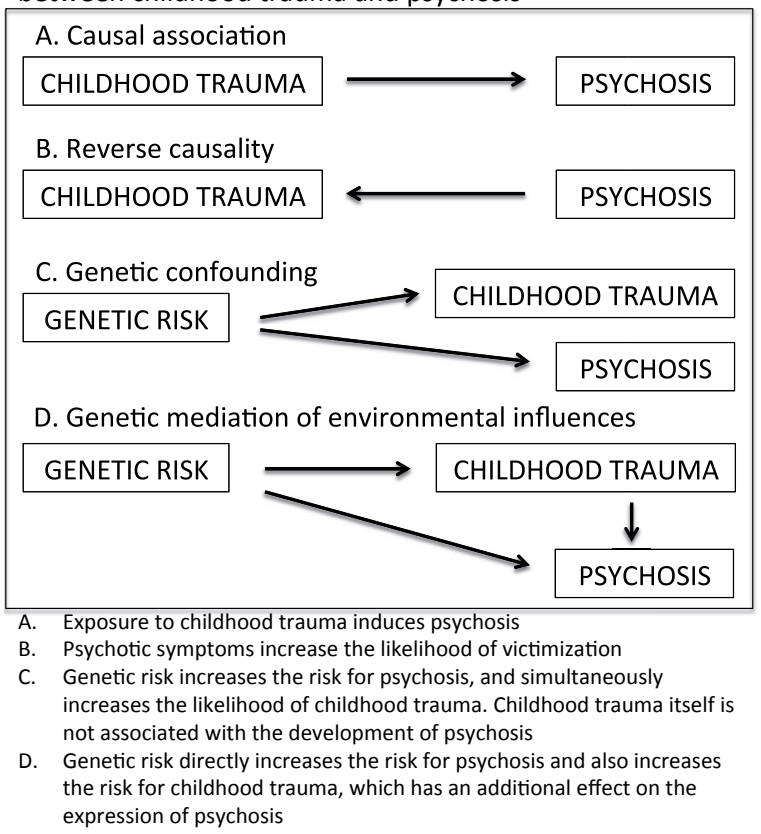

\section{Underlying mechanism: psychology meets biology}

Assuming that the association between childhood trauma and psychosis is indeed causal, are there plausible mechanisms linking the two? Most of the theories on this link postulate a central role for psychological processes ${ }^{21-23}$.

On of these is decreased self-value in relation to others ${ }^{21,22}$. For example, Selten and Cantor-Graae ${ }^{21,22}$ proposed that enduring exposure to a subordinate position or outsider status may be the common denominator linking different environmental factors, including childhood trauma, to risk for psychotic disorder. According to the authors, urban upbringing, minority status, low IQ, hearing impairment and childhood physical or sexual abuse may all conceptually related. They argue that the higher levels of competition in urban environments, the outsider status associated with minority status, fewer job opportunities available for people with lower IQ, social exclusion as a result of hearing impairment, and the humiliation of physical or sexual abuse lead to a state of social defeat. The social defeat theory builds on animal research, showing that social defeat stress, most commonly the stress occasioned by repeated attacks of a stronger intruder animal, leads to abnormalities in dopaminergic neurotransmission ${ }^{24-29}$. Given the well-established role of dopamine in occasioning psychotic symptoms $\mathrm{s}^{30-32}$, the social defeat hypothesis has been considered an appealing theory, linking multiple environmental exposures to risk of psychosis ${ }^{1,33}$. 
Preliminary support for the social defeat theory was recently reported in a study of 38 individuals at ultra-high risk for psychosis. In this study, evidence was found that feelings of a failed struggle and losing rank fuelled negative schemas about the self, which in turn predicted psychotic symptoms ${ }^{34}$.

Other cognitive biases are also likely to play a role in the development of psychotic symptoms. These include jumping to conclusions, difficulties in theory of mind skills, and an attribution style characterized by the tendency to attribute negative events to external factors (especially those that involve the intentional actions of others rather than situational circumstances $)^{35}$. According to the model of Bentall and Fernyhough, early adverse experiences (especially when occurring repeatedly) thus increase the likelihood of negative self-esteem on the one hand and induce an externalizing attributional style on the other ${ }^{23}$. This is exacerbated by problems in understanding others intentions because of difficulties with theory of mind skills. A person may be unable to attribute the negative actions of others to situational circumstances ${ }^{23}$. These factors result in a tendency to anticipate social encounters as a threat, which may persist over time when combined with a cognitive style characterized by drawing conclusions from limited evidence (jumping to conclusions), preventing contextual updating of information, or reality testing ${ }^{23}$.

At the level of the neurobiological substrate, these psychological processes translate to observable changes in the structure or function of brain areas relevant to psychosis. Perhaps the most commonly hypothesized biological mechanism linking childhood adversity to risk of psychosis is sensitization of the mesolimbic dopamine system ${ }^{1,21,36,37}$, resulting in exaggerated dopamine release to subsequent social stressors later in life and possibly also to recreational drugs associated with striatal dopamine release. There are observational reports that may be compatible with trauma-related sensitization at the behavioral level. For example, a study using experience-sampling (a diary method to assess thoughts, emotions and context in the flow of daily life ${ }^{38}$ ) found that individuals that were exposed to childhood trauma reacted more strongly to minor stresses as they occurred in daily life ${ }^{39}$. A 10-year prospective cohort study of 3021 adolescents and young adults from Germany found that childhood trauma and recent life events acted synergistically to induce psychotic symptoms ${ }^{40}$. In addition, replicated evidence suggests that persons exposed to early life adversity have a higher risk for psychosis when they expose take cannabis ${ }^{41-43}$, although not all studies were able to confirm this ${ }^{44}$.

The direct evidence linking early experience to stress-induced dopamine release is limited to the study by Pruessner and colleagues ${ }^{45}$, which reported that a psychosocial stressor induced significant mid-brain dopamine release in individuals reporting low parental care, whereas this was not the case in individuals reporting high levels of care. Moreover, this dopaminergic brain response was significantly correlated $(r=0.78)$, with the salivary cortisol response to stress ${ }^{45}$. In agreement with this finding, both elevated baseline cortisol secretion ${ }^{46-48}$ and a blunted cortisol awakening response $e^{49-51}$ have been reported in patients with psychosis, including those with a history of childhood trauma ${ }^{52}$. Other studies have reported pro-inflammatory alterations in individuals exposed to childhood trauma ${ }^{53-55}$. There is increasing evidence for immune alterations in patients with schizophrenia, 
including replicated evidence of increased IL-2 and IL-6 plasma levels ${ }^{56}$ and replicated, genome-wide significant association at the Major Histocompatibility Complex (MHC) locus $^{57}$. These pro-inflammatory alterations may be especially present in patients with a history of early life adversity ${ }^{58}$ or later exposure to psychosocial stressors ${ }^{58}$, resulting in reduced BDNF mRNA levels, possibly linking early life adversity to brain changes in a number of relevant areas ${ }^{58}$. For example, in patients with first-episode psychosis, childhood trauma may be associated with reduced left hippocampal volume ${ }^{58,59}$ and right and left amygdala volumes ${ }^{59}$, which may underlie the reported worse cognitive performance in schizophrenia patients with childhood trauma compared with their non-traumatized counterparts ${ }^{60,61}$.

\section{Interaction with genetic liability?}

As most of the individuals exposed to childhood trauma do not develop psychotic symptoms, it is commonly accepted that childhood trauma is neither a necessary nor sufficient cause of psychosis and that underlying genetic vulnerability may play an essential role in bringing about psychotic experiences following adversity ${ }^{62,63}$. There is a limited number of studies that have tried to verify this assumption, either using indirect measures of genetic liability, such as cognitive speed ${ }^{64}$, twin modeling ${ }^{7,65}$, parental psychopathology ${ }^{7,66}$, or using direct measures of genetic variation ${ }^{67-69}$.

At the indirect level, Pfeifer and colleagues reported that cognitive speed, a heritable trait associated with psychosis, moderated the effects of childhood trauma on psychotic symptoms in a general population twin sample of 575 female participants ${ }^{64}$. In the same sample, it was similarly shown that a measure of genetic risk for depression, based on the presence of a lifetime diagnosis of depression in the co-twin as well as the twins' genetic relationship (dizygotic/monozygotic), also moderated the effects of trauma on the development of psychotic symptoms ${ }^{65}$. Other studies, however, did not find evidence for an interaction between indirect genetic liability and childhood trauma on the emergence of psychosis ${ }^{7,66}$. In a cohort of Dutch adolescents followed from age 10 to 16 , there was no evidence for an interaction between parental psychopathology and childhood trauma on psychotic symptoms at age 16 or on the developmental course of psychotic symptoms over the follow-up period ${ }^{66}$. Similar findings were reported in the study of Arseneault and colleagues, whether genetic risk was indexed by parental psychopathology or by using the co-twins symptoms as indicator of genetic risk?

Three studies have investigated molecular-genetic interactions with childhood trauma ${ }^{67-69}$. In a sample from the general population, Alemany found that the Val66Met polymorphism of BDNF moderated the effects of trauma on psychotic experiences ${ }^{68}$. Met-carriers showing higher levels of psychotic symptoms than Val/Val individuals following exposure to trauma ${ }^{68}$. Aas and colleagues reported that genetic variation in the serotonin transporter gene may moderate the effects of trauma on cognitive performance, particularly memory, in a sample of 118 patients with a psychotic disorder ${ }^{67}$. Finally, Collip and colleagues investigated interactions between childhood trauma and 4 functional FKBP5 polymor- 
phisms (SNPs) with regard to their effect on psychotic symptoms and cortisol levels in 401 twin individuals from the general population, and in three follow-up samples ( 175 controls, 200 siblings of patients with a psychotic disorder and 195 patients; on psychotic symptoms $)^{69}$. The authors focused on FKBP5 as this gene is a critical modulator of the feedback loop determining glucocorticoid receptor (GR) sensitivity ${ }^{70}$, and FKBP5 SNPs have been consistently associated with development of posttraumatic stress disorder symptoms $\mathrm{s}^{71-74}$ and depression ${ }^{73,75,76}$ in combination with exposure to childhood trauma. The most consistent finding in the Collip study was an interaction between childhood trauma and two FKBP5 SNPs (rs9296158 and rs4713916) on psychotic symptoms and cortisol in the twin sample, combined with a directionally similar interaction in the siblings (rs4713916) or patients (rs9296158) ${ }^{69}$.

While these studies have investigated whether particular variation in the DNA sequence moderates the influence of childhood trauma on psychosis (gene-environment interaction), a second genetic mechanism through which environmental exposures may exert effects on the phenotype of psychosis are epigenetics ${ }^{62,77}$. Epigenetics refers to the reversible regulation of various genomic functions, occurring independently of DNA sequence, mediated through changes in DNA methylation and chromatin structure ${ }^{78}$. Such epigenetic mechanisms have essential functions during development, and allow the long-term regulation of gene function and may mediate environmental effects on gene function. No in vivo studies in humans have yet investigated the possibility that childhood trauma may exert effects on mental outcomes via epigenetic mechanisms. However, animal studies have provided evidence for changes in the methylation of the glucocorticoid NR3C1 receptor associated with the level of post-natal maternal care in rats ${ }^{79}$, and a post-mortem study comparing the hippocampi of suicide victims exposed to childhood abuse to those of healthy controls and suicide victims without a history of abuse reported increased methylation of the NR3C1 promoter, with concomitant changes in $\mathrm{mRNA}^{79}$. It should be noted that a second study failed to replicate this finding ${ }^{79}$. Nevertheless, these findings illustrate the diverse genetic mechanisms through which childhood experiences may mediate the onset of symptoms of different mental disorders, including psychosis ${ }^{62,77}$.

\section{Conclusions and future directions}

Recent, methodologically rigorous studies have provided robust evidence for an association between adverse experiences in childhood and psychosis. Meta-analyses have quantified the association, pointing to ORs in the order of around 3. Prospective studies have shown that reverse causation is unlikely to explain the association, but the current evidence does not exclude it as a possibility. Studies have convincingly shown that the association between childhood trauma and psychosis remains strong and significant when controlling for genetic risk. But, more work is needed to address the possibility of genetic mediation of environmental influences.

Several studies have shown plausible psychological and neurobiological mechanisms linking adverse experiences to psychosis. Psychological processes may induce sensitization 
of the mesolimbic dopamine system, change the stress and immune system and change stress-related brain structures, such as the hippocampus and the amygdala. More work is needed to integrate these findings in more complex models of vulnerability. As the association between childhood trauma and psychosis is not specific but extends to a range of mental disorders, this lack of specificity should be accounted for in any model linking childhood trauma and psychosis. These models should also investigate whether genetic vulnerability plays a role in conferring the mental consequences of adversity, and which genes are likely to be involved. The current, limited evidence, points to genes that are not specifically involved in psychosis but more generally in regulating mood (serotonin transporter gene), neuroplasticity (BDNF) and stress-reactivity (FKBP5), in keeping with an effect of childhood trauma on a range of mental disorders.

\section{Acknowledgments}

The Canadian Psychiatric Association supports the In Review series by providing an honorarium to the authors. The research leading to these results has received funding from the European Community's Seventh Framework Program under grant agreement No. HEALTH-F2-2009-241909 (Project EU-GEI). 


\section{REFERENCES}

1. van Winkel R, Stefanis NC, MyinGermeys I. Psychosocial stress and psychosis. A review of the neurobiological mechanisms and the evidence for gene-stress interaction. Schizophr Bull. 2008;34(6):1095-1105.

2. Bendall $\mathrm{S}$, Jackson $\mathrm{HJ}$, Hulbert $\mathrm{CA}$, et al. Childhood trauma and psychotic disorders: a systematic, critical review of the evidence. Schizophr Bull. 2008;34(3):568-579.

3. Read J, van Os J, Morrison AP, et al. Childhood trauma, psychosis and schizophrenia: a literature review with theoretical and clinical implications. Acta Psychiatr Scand. 2005;112(5):330-350.

4. Morgan C, Fisher H. Environmental factors in schizophrenia: Childhood trauma -- a critical review. Schizophr Bull. 2007;33(1):3-10.

5. Heins $M$, Simons $C$, Lataster $T$, et al. Childhood trauma and psychosis: a case-control and case-sibling comparison across different levels of genetic liability, psychopathology, and type of trauma. Am J Psychiat. 2011;168(12):1286-1294.

6. Cutajar M, Mullen P, Ogloff J, et al. Schizophrenia and other psychotic disorders in a cohort of sexually abused children. Arch Gen Psychiat. 2010;67:1114-1119.

7. Arseneault $L$, Cannon $M$, Fisher $H$, et al. Childhood trauma and children's emerging psychotic symptoms: a genetically sensitive longitudinal cohort study. Am J Psychiat. 2011;168:65-72.

8. Fisher $\mathrm{HL}$, Jones $\mathrm{PB}$, Fearon $\mathrm{P}$, et al. The varying impact of type, timing and frequency of exposure to childhood adversity on its association with adult psychotic disorder. Psychol Med. 2010;40:1967-1978.

9. Freeman D, Fowler D. Routes to psychotic symptoms: trauma, anxiety and psychosis-like experiences. Psychiatr Res. 2009;169:107-112.

10. Steel C, Marzillier S, Fearon P, et al. Childhood abuse and schizotypal personality. Soc Psych Psych Epidem. 2009;44:917-923.

11. Galletly $C$, van Hooff M, McFarlane A. Psychotic symptoms in young adults exposed to childhood trauma - A 20 year follow-up study. Schizophr Res. 2011;127:76-82.

12. Varese F, Smeets F, Drukker M, et al. Childhood adversities increase the risk of psychosis: a meta-analysis of patient-control, prospective and cross-sectional cohort studies. Schizophr Bull. 2012:doi:10.1093/ schbul/sbs1050.

13. Matheson S, Shepherd A, Pinchbeck RM, et al. Childhood adversity in schizophrenia: a systematic metaanalysis. Psychol Med. 2012: 1-13.

14. Dean K, Stevens H, Mortensen PB, et al. Full spectrum of psychiatric outcomes among offspring with parental history of mental disorder. Arch Gen Psychiat. 2010;67(8):822-829.

15. Mortensen PB, Pedersen MG, Pedersen CB. Psychiatric family history and schizophrenia risk in Denmark: which mental disorders are relevant? Psychol Med. 2010;40(2):201-210.

16. van Os J, Rutten BP, Poulton R. Gene-environment interactions in schizophrenia: review of epidemiological findings and future directions. Schizophr Bull. 2008;34(6):1066-1082. 
17. Wigman JTW, van Winkel $R$, Raaijmakers QAW, et al. Evidence for a persistent, deteriorating subtype of subclinical psychotic experiences: a six-year longitudinal general population study. Psychol Med. 2011;41(11):2317-2329.

18. Schreier A, Wolke D, Thomas K, et al. Prospective study of peer victimization in childhood and psychotic symptoms in a nonclinical population at age 12 years. Arch Gen Psychiat. 2009;66(527-536).

19. Husted JA, Ahmed R, Chow EWC, et al. Childhood trauma and genetic factors in familial schizophrenia associated with the NOS1AP gene. Schizophr Res. 2010;121:187-192.

20. Alemany $S$, Goldberg $X$, van Winkel $R$, et al. Childhood adversity and psychosis: examining whether the association is due to genetic confounding using a monozygotic twin differences approach. Eur Psychiatry. 2012:in press.

21. Selten JP, Cantor-Graae E. Social defeat: risk factor for schizophrenia? Br J Psychiatry. 2005;187:101-102.

22. Selten JP, Cantor-Graae E. Hypothesis: social defeat is a risk factor for schizophrenia? Br J Psychiatry Suppl. 2007;51:s9-12.

23. Bentall R, Fernyhough C. Social predictors of psychotic experiences: specificity and psychological mechanisms. Schizophr Bull. 2008;34(6):1012-1020.

24. Abercrombie ED, Keefe KA, DiFrischia DS, et al. Differential effect of stress on in vivo dopamine release in striatum, nucleus accumbens, and medial frontal cortex. J Neurochem. 1989;52(5):1655-1658.
25. Finlay JM, Zigmond MJ. The effects of stress on central dopaminergic neurons: possible clinical implications. Neurochem Res. 1997;22(11):1387-1394.

26. King D, Zigmond MJ, Finlay JM. Effects of dopamine depletion in the medial prefrontal cortex on the stress-induced increase in extracellular dopamine in the nucleus accumbens core and shell. Neuroscience. 1997;77(1):141-153.

27. Krishnan V, Han MH, Graham DL, et al. Molecular adaptations underlying susceptibility and resistance to social defeat in brain reward regions. Cell. 2007;131(2):391-404.

28. Krishnan $\mathrm{V}$, Han $\mathrm{MH}$, Mazei-Robison $M$, et al. AKT signaling within the ventral tegmental area regulates cellular and behavioral responses to stressful stimuli. Biol Psychiatry. 2008;64(8):691-700.

29. Cao JL, Covington HE, 3rd, Friedman $A K$, et al. Mesolimbic dopamine neurons in the brain reward circuit mediate susceptibility to social defeat and antidepressant action. J Neurosci. 2010;30(49):16453-16458.

30. Howes $\mathrm{O}$, Fusar-Poli P, Bloomfield M, et al. From the prodrome to chronic schizophrenia: the neurobiology underlying psychotic symptoms and cognitive impairments. Curr Pharm Des. 2012;18(4):459-465.

31. Howes O, Bose SK, Turkheimer F, et al. Dopamine synthesis capacity before onset of psychosis: a prospective (18F)-DOPA PET imaging study. Am J Psychiat. 2011;168(2):1311-1317.

32. Miyake N, Thompson J, Skinbjerg M, et al. Presynaptic dopamine in 
schizophrenia. CNS Neurosci Ther. 2011;17(2):104-109.

33. Morgan $\mathrm{C}$, Charalambides $\mathrm{M}$, Hutchinson $\mathrm{G}$, et al. Migration, ethnicity, and psychosis: toward a sociodevelopmental model. Schizophr Bull. 2010;36(4):655-664.

34. Stowkowy J, Addington J. Maladaptive schemas as a mediator between social defeat and positive symptoms in young people at clinical high risk for psychosis. Early Interv Psychiatry. 2012;6(1):87-90.

35. Bentall R, Rowse G, Shryane N, et al. The cognitive and affective structure of paranoid delusions: a transdiagnostic investigation of patients with schizophrenia spectrum disorders and depression. Arch Gen Psychiatry. 2009;66(3):236-247.

36. Collip D, Myin-Germeys I, Van Os J. Does the concept of "sensitization" provide a plausible mechanism for the putative link between the environment and schizophrenia? Schizophr Bull. 2008;34(2):220-225.

37. Howes OD, McDonald C, Cannon M, et al. Pathways to schizophrenia: the impact of environmental factors. Int J Neuropsychopharmacol. 2004;7 Suppl 1:S7-S13.

38. Myin-Germeys I, Oorschot M, Collip $D$, et al. Experience sampling research in psychopathology: opening the black-box of daily life. Psychol Med. 2009;39(9):1533-1547.

39. Lardinois $M$, Lataster $T$, Mengelers $R$, et al. Childhood trauma and increased stress sensitivity in psychosis. Acta Psychiatr Scand. 2010:DOI: 10.1111/j.1600-0447.2010.01594.x.

40. Lataster J, Myin-Germeys I, Lieb R, et al. Adversity and psychosis: a 10-year prospective study investigating synergism between early and recent adversity in psychosis. Acta Psychiatr Scand. 2012;125(5):388-399.

41. Harley M, Kelleher I, Clarke M, et al. Cannabis use and childhood trauma interact additively to increase the risk of psychotic symptoms in adolescence. Psychol Med. 2010;40:1627-1634.

42. Houston JE, Murphy JP, Adamson G, et al. Childhood sexual abuse, early cannabis use, and psychosis: testing an interaction model based on the National Comorbidity Survey. Schizophr Bull. 2008;34(3):580-585.

43. Konings M, Stefanis N, Kuepper R, et al. Replication in two independent population-based samples that childhood maltreatment and cannabis use synergistically impact on psychosis risk. Psychol Med. 2012;42(1):149-159.

44. Kuepper R, Henquet C, Lieb R, et al. Non-replication of interaction between cannabis use and trauma in predicting psychosis. Schizophr Res. 2011;131(1-3):262-263.

45. Pruessner JC, Champagne F, Meaney $\mathrm{MJ}$, et al. Dopamine release in response to a psychological stress in humans and its relationship to early life maternal care: a positron emission tomography study using [11C] raclopride. J Neurosci. 2004;24(11):2825-2831.

46. Ryan MC, Sharifi N, Condren R, et al. Evidence of basal pituitary-adrenal overactivity in first episode, drug naive patients with schizophrenia. Psychoneuroendocrinology. 2004;29(8):1065-1070.

47. Gallagher P, Watson $S$, Smith MS, et al. Plasma cortisol-dehydroepiandros- 
terone (DHEA) ratios in schizophrenia and bipolar disorder. Schizophr Res. 2007;90:258-265.

48. Ritsner M, Gibel A, Maayan R, et al. State and trait related predictors of serum cortisol to DHEA(S) molar ratios and hormone concentrations in schizophrenia patients.

Eur Neuropsychopharmacol. 2007;17:257-264.

49. Pruessner M, Boekestyn L, BechardEvans $L$, et al. Sex differences in the cortisol response to awakening in recent onset psychosis.

Psychoneuroendocrinology. 2008;33:1151-1154.

50. Mondelli V, Dazzan $P$, Hepgul N, et al. Abnormal cortisol levels during the day and cortisol awakening response in first-episode psychosis: the role of stress and of antipsychotic treatment. Schizophr Res. 2010;116:234-242.

51. Pruessner M, Vracotas $N$, Joober R, et al. Blunted cortisol awakening response in men with first episode psychosis: relationship to parental bonding. Psychoneuroendocrinology. 2012:http://dx.doi.org/10.1016/j. psyneuen.2012.1006.1002.

52. Braehler C, Holowka D, Brunet A, et al. Diurnal cortisol in schizophrenia patients with childhood trauma. Schizophr Res. 2005;79:353-354.

53. Pace $T$, Mletzko $T$, Alagbe $O$, et al. Increased stress-induced inflammatory responses in male patients with major depression and increased early life stress. Am J Psychiat. 2006;163:1630-1633.

54. Danese A, Moffitt TE, Pariante CM, et al. Elevated inflammation levels in depressed adults with a history of childhood treatment. Arch Gen Psychiat. 2008;65:409-415.
55. Carpenter L, Tyrka A, Ross N, et al. Association between plasma IL- 6 response to acute stress and early-life adversity in healthy adults. Neuropsychopharmacology. 2010;35:2617-2623.

56. Potvin S, Stip E, Sepehry A, et al. Inflammatory cytokine alterations in schizophrenia: a systematic quantitative review. Biol Psychiatry. 2008;63(801-808).

57. Ripke S, Sanders AR, Kendler KS, et al. Genome-wide association study identifies five new schizophrenia loci. Nat Genet. 2011;43(10):969-976.

58. Mondelli V, Cattaneo A, Belvederi Murri $M$, et al. Stress and inflammation reduce brain-derived neurotrophic factor expression in first-episode psychosis: a pathway to smaller hippocampal volume. J Clin Psychiatry. 2011;72(12):1677-1684.

59. Hoy K, Barrett S, Shannon C, et al. Childhood Trauma and Hippocampal and Amygdalar Volumes in FirstEpisode Psychosis. Schizophr Bull. 2011.

60. Aas M, Dazzan P, Fisher HL, et al. Childhood trauma and cognitive function in first-episode affective and non-affective psychosis. Schizophr Res. 2011;129(1):12-19.

61. Shannon C, Douse K, McCusker C, et al. The association between childhood trauma and memory functioning in schizophrenia. Schizophr Bull. 2011;37(3):531-537.

62. van Winkel R, Esquivel G, Kenis G, et al. Genome-wide findings in schizophrenia and the role of gene-environment interplay. CNS Neurosci Ther. 2010;16:e185-e192 
63. Van Os J, Kenis G, Rutten B. The environment and schizophrenia. Nature. 2010;468(7321):203-212.

64. Pfeifer S, Krabbendam L, Myin-Germeys I, et al. A cognitive intermediate phenotype study confirming possible gene-early adversity interaction in psychosis outcome: a general population twin study. Psychosis. 2010;2(1): 1-11.

65. Kramer IM, Simons C, Myin-Germeys I, et al. Evidence that genes for depression impact on the pathway from trauma to psychotic-like symptoms by occasioning emotional dysregulation. Psychol Med. 2011:doi:10.1017/ S0033291711001474

66. Wigman JTW, van Winkel R, Ormel J, et al. Early trauma and familial risk in the development of the extended psychosis phenotype in adolescence. Acta Psychiatr Scand. 2012;126(4):266-273.

67. Aas M, Djurovic S, Athanasiu L, et al. Serotonin transporter gene polymorphisms, childhood trauma, and cognitiion in patients with psychotic disorders. Schizophr Bull. 2012;38(1):2012.

68. Alemany $S$, Arias $M$, Aguilera $M$, et al. Childhood abuse, the BDNF Val66Met polymorphism and adult psychotic-like experiences. Br J Psychiat. 2011;199(38-42).

69. Collip D, Myin-Germeys I, Wichers M, et al. FKBP5 as a possible moderator of the psychosis-inducing effects of childhood trauma across different levels of familial liability. Br J Psychiat. submitted.

70. Binder EB. The role of FKBP5, a co-chaperone of the glucocorticoid receptor in the pathogenesis and therapy of affective and anxiety disorders. Psychoneuroendocrinology. 2009;34S1:S186-S195.

71. Mehta $\mathrm{D}$, Gonik $\mathrm{M}$, Klengel $\mathrm{T}$, et al. Using polymorphisms in FKBP5 to define biologically distinct subtypes of posttraumatic stress disorder: evidence from endocrine and gene expression studies. Arch Gen Psychiatry. 2011;68(9):901-910.

72. Binder EB, Bradley RG, Liu W, et al. Association of FKBP5 polymorphisms and childhood abuse with risk of posttraumatic stress disorder symptoms in adults. Jama. 2008;299(11):1291-1305.

73. Koenen KC, Uddin M. FKBP5 polymorphisms modify the effects of childhood trauma.

Neuropsychopharmacology. 2010;35(8):1623-1624.

74. Xie $P$, Kranzler HR, Poling J, et al. Interaction of FKBP5 with childhood adversity on risk for post-traumatic stress disorder.

Neuropsychopharmacology. 2010;35(8):1684-1692.

75. Zimmermann P, Bruckl T, Nocon A, et al. Interaction of FKBP5 gene variants and adverse life events in predicting depression onset: results from a 10-year prospective community study. Am J Psychiat. 2011;168(10):1107-1116.

76. Appel K, Schwan C, Mahler J, et al. Moderation of adult depression by a polymorphism in the FKBP5 gene and childhood physical abuse in the general population. Neuropsychopharmacology. 2011;36(10):1982-1991. 
77. Rutten BP, Mill J. Epigenetic mediation of environmental influences in major psychotic disorders. Schizophr Bull. 2009;35(6):1045-1056.

78. Jaenisch R, Bird A. Epigenetic regulation of gene expression: how the genome integrates intrinsic and environmental signals. Nat Genet. 2003;33 Suppl:245-254.

79. Weaver IC, Cervoni N, Champagne F, et al. Epigenetic programming by maternal behavior. Nat Neurosci. 2004;7(8):847-854. 


\title{
CHAPTER 6
}

\section{Does Social Defeat Mediate the Association Between Childhood Trauma and Psychosis? Evidence from the Nemesis-2 Study}

\author{
Martine van Nierop ${ }^{A}$ \\ Jim van $O s^{A B}$ \\ Nicole Gunther ${ }^{C}$ \\ Catherine van Zelst ${ }^{\mathrm{A}}$ \\ Ron de Graaf ${ }^{D}$ \\ Margreet ten Have ${ }^{\mathrm{D}}$ \\ Saskia van Dorsselaer ${ }^{\mathrm{D}}$ \\ Maarten Bak ${ }^{\mathrm{A}}$ \\ Inez Myin-Germeys ${ }^{A}$ \\ Ruud van Winkel ${ }^{\mathrm{AE}}$
}

\footnotetext{
A Dept. of Psychiatry and Psychology, School for Mental Health and Neuroscience, EURON, Maastricht University Medical Centre, PO Box 616 (Vijv1), 6200 MD Maastricht, The Netherlands

${ }^{B}$ King's College London, King's Health Partners, Department of Psychosis Studies Institute of Psychiatry, London, UK

c School of Psychology, Open University, The Netherlands

${ }^{D}$ Netherlands Institute of Mental Health and Addiction, Da Costakade 45, 3521 VS Utrecht, The Netherlands

E University Psychiatric Center Katholieke Universiteit Leuven, campus Kortenberg, Leuvensesteenweg, Kortenberg, Belgium
} 


\section{ABSTRACT}

\section{Objective}

Based on theoretical considerations and animal studies mediation of 'social defeat' (SD) in the association between childhood trauma (CT) and psychosis was investigated.

\section{Methods}

Trained interviewers administered a structured interview assessing CT, psychotic experiences and other psychopathology in 6646 participants in the second Netherlands Mental Health Survey and Incidence Study (NEMESIS-2).

\section{Results}

CT was associated with psychotic experiences making up the extended psychosis phenotype (EPP), as well as with a diagnosis of psychotic disorder (PD). Similarly, CT was associated with a priori selected items indexing SD (discouraged, hopeless, worthless, loss of selfconfidence, low self-esteem, better off dead, suicidal thoughts) and with a measure of affective dysregulation (AD), which in turn were also associated with psychosis. While SD and $A D$ individually acted as mediators in the association between CT and EPP, only SD acted as a mediator in the association between CT and PD. Cannabis use did not mediate the association between $\mathrm{CT}$ and EPP or PD.

\section{Conclusion}

The present results suggest a developmental model implicating SD as an important mediator in the link between childhood adverse experiences and later development of psychotic experiences. The combined mediation by SD and AD is compatible with an 'affective pathway' to early psychosis. 


\section{INTRODUCTION}

Childhood trauma (CT) is one of the most extensively studied environmental factors associated with the development of psychosis ${ }^{1-3}$. Associations have been reported between $\mathrm{CT}$ and psychotic disorder ${ }^{4,5}$, psychotic experiences ${ }^{6-8}$, and schizotypy ${ }^{9}$. There is evidence for a dose-response relationship ${ }^{10,11}$ and increased severity of psychotic experiences in adulthood after $\mathrm{CT}^{12,13}$. In a recent extensive meta-analysis, including 41 studies and over 80,000 respondents, examining the association between $\mathrm{CT}$ and psychosis, an overall odds ratio of $2.78(95 \% \mathrm{Cl} 2.34-3.31)$ was found ${ }^{14}$.

Different types of CT have been investigated, suggesting that trauma in the areas of sexual abuse $^{4}$ or peer victimization ${ }^{11}$, which have a clear intentional component, may have stronger associations with psychosis than trauma with unintentional harm ${ }^{6}$.

However, epidemiological studies with larger sample sizes commonly used self-report measures of psychotic experiences ${ }^{7}$, or had interview-based validation available for only a small part of the sample ${ }^{12,15}$. In earlier studies it was found that self-reports of psychotic experiences generate high rates of false positives ${ }^{16,17}$, pointing to the importance of interview-based validation conducted by trained interviewers.

Even if the observed association between CT and psychosis is assumed to be causal, its nature in terms of underlying mechanisms remains incompletely understood. Some possible psychological mechanisms that have been postulated include deficits in source monitoring or an overestimation of personal threat ${ }^{18}$, or insecure attachment styles ${ }^{19}$. Alternatively, guided by animal studies reporting associations between induction of social defeat stress on the one hand and depression-related phenotypes and dopaminergic abnormalities on the other ${ }^{20,21}$, Selten and Cantor-Graae hypothesized that several known environmental risk factors for psychosis, such as outsider status through migration ${ }^{22}$ and different forms of psychological, emotional, physical and sexual abuse may cause feelings of outsider status and reduced value, referred to as 'social defeat'. Social defeat, in turn, may increase risk for psychosis ${ }^{23}$. Preliminary support for this theory was recently reported in a study of 38 individuals at ultra high risk for psychosis ${ }^{24}$. In this study, evidence was found that feelings of a failed struggle and losing rank, fuelled negative schemas about the self, which in turn predicted psychotic symptoms. Similarly, Taylor and colleagues found a significant association between perceptions of defeat and positive psychotic symptoms in a sample of 78 schizophrenic outpatients ${ }^{25}$. Thus, the theory that the experience of 'social defeat' mediates the association between $\mathrm{CT}$ and psychosis is worthy of further investigation ${ }^{26}$.

Other potential mediators of the association between CT and psychosis should also be considered. For example, several studies report evidence that affective dysregulation directly exacerbates psychotic phenomena and increases the risk of psychotic disorder ${ }^{27,28}$. As CT is associated with affective dysregulation ${ }^{29}$, depression should be accounted for in any study examining the association between $\mathrm{CT}$ and psychosis. Similarly, cannabis use is more prevalent in individuals that have experienced $\mathrm{CT}^{30}$. This is relevant, given the wellestablished association between cannabis use and psychosis ${ }^{31}$. 


\section{Aims of the study}

The current study, therefore, aimed to investigate to what degree the association between childhood trauma and psychotic experiences is mediated by social defeat, as well as by cannabis use and affective dysregulation. The risk set was a large, population-based sample using lay interview measures and additional follow-up interviews, both at the level of subclinical psychotic experiences that constitute the extended psychosis phenotype in the general population, and at the level of psychotic disorder.

\section{METHODS}

\section{NEMESIS-2}

This study forms part of the recent second Netherlands Mental Health Survey and Incidence Study (NEMESIS-2), a longitudinal study of the prevalence, incidence, course and consequences of psychiatric disorders in the Dutch general population. NEMESIS-2 replicates and extends the first NEMESIS-1 study, conducted from 1996 to 199932, in an independent, non-overlapping sample. The study was approved by the Medical Ethics Review Committee for Institutions on Mental Health Care. Participants were selected based on a multistage random sampling procedure, resulting in a sample that was representative of the Dutch adult population in terms of age, region and population density ${ }^{33}$. Participants were included between the ages of 18-65, insufficient fluency in Dutch was an exclusion criterion. The participants were interviewed at home by trained interviewers, who were not clinicians, with the Composite International Diagnostic Interview (CIDI) version 3.0 34, 35 , and questionnaires. A more detailed description of NEMESIS-2 methodology was presented by De Graaf and colleagues ${ }^{33,36}$.

\section{Childhood trauma}

Childhood trauma was assessed using a questionnaire based on the NEMESIS-1 trauma questionnaire ${ }^{33}$. Whenever a subject reported having experienced one of five types of CT (emotional neglect [not listened to, ignored or unsupported], physical abuse [kicked, hit, bitten or hurt with object or hot water], psychological abuse [yelled at, insulted, unjustly punished/treated, threatened, belittled or blackmailed], sexual abuse [any unwanted sexual experience] and peer victimization [any bullying]; before the age of 16), they were asked to state how often it had occurred, on a scale of 1 (once) to 5 (very often). Factor analysis indicated that the five CT scales loaded onto one factor (eigenvalue 2.47); therefore one CT scale (range 0-25) was constructed consisting of the sum of the five types of CT.

\section{Psychosis}

Studies on earlier CIDI versions concluded that the instrument provides a reliable and valid assessment of mental disorders, with the exception of psychosis ${ }^{37}$. As CIDI 
methodology to assess psychotic experiences in CIDI 1.0 and CIDI 2.0 was not included in CIDI 3.0, a psychosis add-on instrument was constructed, based on the section of psychotic symptoms in CIDI versions 1.0 and 2.0. This part of the interview consisted of questions regarding 20 lifetime psychotic experiences, each rated 'yes', 'no', 'don't know' or 'refuse'. The 20 items consisted of 15 delusional experiences and 5 hallucinatory experiences (see

${ }^{17}$ for a detailed description). As clinical relevance of psychotic experiences may be difficult to diagnose by lay interviewers ${ }^{38}$, and the interviewers made no clinical judgment about participants' answers, the reported experiences may be considered a more accurate version of 'self-report'.

Individuals who endorsed at least one lifetime psychotic experience (1084 out of 6646 participants) were contacted for reinterview over the telephone by an experienced clinician at the level of psychologist or psychiatrist within eight weeks after the initial interview, similar to the procedure in NEMESIS- ${ }^{12}$. Reinterviews were conducted using questions from the Structured Clinical Interview for DSM-IV (SCID-I), an instrument with proven reliability and validity in diagnosing psychotic disorders ${ }^{39}$. Findings from all reinterviews were discussed with a second clinician (MB).

Psychotic experiences were considered present when the psychotic nature of at least one of the self-reported psychotic experiences was confirmed at the follow-up interview (hereafter: Extended Psychosis Phenotype ${ }^{40}$ [EPP] group, $\mathrm{N}=384$ ). Clinical diagnoses of psychotic disorder (schizophrenia or schizophreniform disorder) were based on both the self-report part of the questionnaire and on the follow-up interview (Psychotic Disorder [PD] group, $\mathrm{N}=43$ ). These two samples were overlapping: all participants in the PD group were also part of the EPP group.

\section{Psychosis severity}

Whenever a psychotic experience was endorsed, the subject was asked to state, on a 1 (rarely) to 4 (almost always) scale, how often this experience occurred (frequency), how much it bothered them (distress) and to what extent the experience had an influence on their daily professional and social activities (impact). Total frequency, distress and impact scores were calculated as the mean of the scores of these items across the psychotic experiences. As factor analysis indicated that the three aspects of severity loaded onto one factor (eigenvalue 1.86), the three severity items were summed to a psychosis severity scale (range 0-12) for both the severity of psychotic experiences in the extended psychosis phenotype [i.e. EPP severity] and the severity of psychotic experiences in the context of a diagnosis of psychotic disorder [i.e. PD severity]. 


\section{Social defeat}

Participants were asked if they had ever experienced an episode of a number of consecutive days during which they had felt low. If endorsed, participants were asked if, during this period, they had experienced ten symptoms indexing self-devaluation, feelings of worthlessness and hopelessness for the future, which were $a$ priori selected to represent the concept of 'social defeat' based on theoretical considerations ${ }^{23}$. These were: (i) being discouraged about things going on in life; (ii) hopeless about the future; (iii) having loss of self-confidence; (iv) feeling less than other people; (v) feeling worthless; (vi) considering oneself better off dead; (vii) thinking about suicide; (viii) repeatedly thinking about death; (ix) making suicidal plans; $(x)$ making a suicidal attempt. Factor analysis indicated that seven of the initially selected ten symptoms strongly loaded onto a single factor (items $i$ through vii; eigenvalue 3.7), while three suicide-related items (viii, ix and x) loaded onto a separate factor. Therefore, the 7 items loading onto a single factor were summed to constitute a social defeat scale (range 0-7).

\section{Affective symptoms}

As described above, participants were asked if they had ever experienced an episode lasting a number of consecutive days during which they had felt low. If endorsed, subjects were asked if, during this period, they had experienced any of 21 general affective symptoms. These were: (i) feeling sad for several consecutive days; (ii) loss of interest; (iii) diminished pleasure; (iv) problems with concentration; (v) difficulty making decisions; (vi) feeling guilty; (vii) irritability; (viii) feeling nervous or anxious; (ix) panic attacks; (x) repeatedly thinking about death; (xi) suicide plans; (xii) suicide attempt; (xiii) overwhelmed by daily responsibilities; (xiv) preferring to be alone; (xv) reduced motivation to talk; (xvi) crying; (xvii) loss or increase in appetite; (xviii) change in sleep pattern; (xix) change in energy; ( $x x$ ) slower or agitated movements; (xxi) slower or erratic thoughts. Factor analysis indicated that these items loaded onto two factors (eigenvalues 3.9 and 1.6); however the three items that loaded onto the second factor (thinking about death, suicide plans and suicide attempt) also loaded on the first factor, together with all the other items. Thus, a general affective dysregulation score was obtained by summing the 21 affective symptoms. 


\section{Cannabis use}

Cannabis use was assessed in the section Illegal Substance Use of the CIDI 3.0. Subjects were asked to state whether they had ever used cannabis, and, if so, were rated on frequency of use in the period of most frequent use on a scale of 1 (less than once per month) to 6 (every day).

\section{Sample and prevalences}

The total sample consisted of 6646 participants (response rate 65\%), with a mean age of 44, of which $45 \%$ was male. 1084 individuals endorsed a 'self-reported' psychotic experience (16\%). From this sample, 792 subjects participated in the reinterview (participation rate: 73\%). No significant differences existed between those that participated in the reinterview and those that did not with regard to age, lifetime Axis I disorder, sex, educational level or employment status. These similarities notwithstanding, non-participants more often had self-reports of psychotic experiences (mean $=2.5$ [95\% Cl: $2.3-2.8]$ ) compared to participants (mean $=1.9[95 \% \mathrm{Cl}: 1.8-2.0]$ ). For full sample characteristics see Table 1 . 
Table 1 NEMESIS-2 baseline sample characteristics and non-weighted prevalences

\begin{tabular}{|c|c|c|c|c|c|c|}
\hline & \multicolumn{2}{|l|}{$\begin{array}{l}\text { Controls }^{\mathrm{F}} \\
(\mathrm{N}=5868)\end{array}$} & \multicolumn{2}{|c|}{$\begin{array}{l}\text { Extended psychosis } \\
\text { phenotype group } \\
(\mathrm{N}=384)\end{array}$} & \multicolumn{2}{|c|}{$\begin{array}{l}\text { Psychotic disorder group } \\
(\mathrm{N}=43)\end{array}$} \\
\hline & $\mathbf{N}$ & $\%$ & $\mathbf{N}$ & $\%$ & $\mathbf{N}$ & $\%$ \\
\hline Men & 2672 & 46 & 138 & 36 & 17 & 40 \\
\hline Paid job & 4422 & 75 & 266 & 69 & 20 & 47 \\
\hline No or primary education & 273 & 5 & 26 & 7 & 3 & 7 \\
\hline Lower secondary education & 1595 & 27 & 104 & 27 & 18 & 42 \\
\hline Higher secondary education & 1864 & 32 & 143 & 37 & 12 & 28 \\
\hline $\begin{array}{l}\text { Higher professional } \\
\text { education/university }\end{array}$ & 2136 & 36 & 111 & 29 & 10 & 23 \\
\hline $\begin{array}{l}\text { Ethicity } \\
\text { - White European }\end{array}$ & 5389 & 92 & 340 & 89 & 31 & 72 \\
\hline \multirow[t]{2}{*}{ - Other } & 479 & 8 & 44 & 11 & 12 & 28 \\
\hline & $\begin{array}{l}\text { Mean } \\
\text { (min-max) }\end{array}$ & SD & $\begin{array}{l}\text { Mean } \\
\text { (min-max) }\end{array}$ & SD & $\begin{array}{l}\text { Mean } \\
\text { (min-max) }\end{array}$ & SD \\
\hline Age & $44(18-65)$ & 12.4 & $43(18-64)$ & 13.1 & $41(18-63)$ & 12.9 \\
\hline Childhood trauma $^{A}$ & $1.4(0-24)$ & 2.9 & $3.9(0-24)$ & 4.75 & $5.8(0-19)$ & 4.8 \\
\hline Bullying ${ }^{B}$ & $0.4(0-5)$ & 1.0 & $0.9(0-5)$ & 1.6 & $1.9(0-5)$ & 1.8 \\
\hline Emotional abuse $^{\mathrm{B}}$ & $0.4(0-5)$ & 1.0 & $1.1(0-5)$ & 1.6 & $1.5(0-5)$ & 1.9 \\
\hline Psychological abuse ${ }^{B}$ & $0.4(0-5)$ & 1.0 & $1.0(0-5)$ & 1.5 & $1.6(0-5)$ & 1.7 \\
\hline Physical abuse $^{B}$ & $0.2(0-5)$ & 0.7 & $0.6(0-5)$ & 1.2 & $0.8(0-5)$ & 1.4 \\
\hline Sexual abuse ${ }^{B}$ & $0.1(0-5)$ & 0.5 & $0.4(0-5)$ & 0.9 & $0.4(0-3)$ & 0.8 \\
\hline Social defeat ${ }^{c}$ & $0.8(0-7)$ & 1.8 & $2.0(0-7)$ & 2.6 & $4.3(0-7)$ & 2.9 \\
\hline Affective dysregulation ${ }^{D}$ & $2.7(0-21)$ & 5.3 & $6.5(0-21)$ & 7.3 & $12.4(0-21)$ & 7.4 \\
\hline Cannabis use ${ }^{\mathrm{E}}$ & $0.4(0-6)$ & 1.0 & $0.7(0-6)$ & 1.6 & $1.2(0-6)$ & 2.0 \\
\hline
\end{tabular}

A Childhood trauma scale (range 0-25): 5 types of trauma combined

B Score range 0 (never) -5 (very often)

c Score range $0-7$ (expressing number of social defeat items - discouraged, hopeless, worthless, loss of self-confidence, low self-esteem, better off dead, suicidal thoughts)

D Score range 0-21 (expressing number of general affective dysregulation items)

E Frequency of use in the period of most frequent use on a scale of 0 (never) to 6 (every day)

F Participants with no self-reported psychotic experiences

${ }^{G}$ Participants with at least one validated psychotic experience (self-reported psychotic experience confirmed at follow-up interview) 


\section{Analyses}

All analyses were performed using STATA, version $11^{41}$. The level of significance $(\alpha)$ is set at 0.05 .

\section{Presence of psychosis}

To examine the association between $\mathrm{CT}$ and dichotomous presence of psychosis, irrespective of its severity, logistic regression analyses were applied using any psychotic experience (vs no psychotic experiences) and PD as respective dichotomous dependent variables. Covariates included in the analyses were CT, age, sex, cannabis use ${ }^{42}$, and affective dysregulation ${ }^{43}$. Additional analyses were performed using the scales of each separate type of CT as covariates.

\section{Severity of psychosis}

In order to examine the association between CT and continuous severity of psychosis (EPP and PD severity), linear regression analyses were applied. Covariates included in the analyses were $\mathrm{CT}$, age, sex, cannabis use and affective dysregulation. As there may be gender differences in the association of childhood trauma and psychosis ${ }^{44}$, separate analyses were performed in which an interaction term of gender and trauma was added to the analysis. This was done at the level of the EPP but not at the level of PD, as the groups with psychotic disorder were too small for meaningful subgroup analyses (14 males and 19 females with presence of PD and CT).

\section{Mediation models}

In order to investigate whether and how social defeat, affective dysregulation and cannabis use mediated the association between $\mathrm{CT}$ and psychosis, several analyses were performed.

First, associations between $\mathrm{CT}$ and social defeat, and social defeat and psychosis severity were investigated using linear regression. The same was done for other potential mediators of the association between CT and psychosis, i.e. cannabis use $\mathrm{e}^{30}$ and affective dysregulation ${ }^{45}$. Furthermore, additional analyses were performed including the interaction term with gender, at the level of the EPP.

Second, in order to investigate mediation, each of the potential mediators (cannabis use, social defeat and affective dysregulation) was added to the model of CT predicting psychosis severity, separately at the level of the EPP and at the level of PD. Each variable that $a$ priori (i) diminished the standardized effect size ( $\beta$ ) of CT with more than $10 \%$ and (ii) showed a significant association with psychosis in this model was selected for further mediation analyses. The variables that diminished the effect size of CT with less than $10 \%$ or did not show a significant association with psychosis would be added as a confounder 
in further analyses. In case of more than one identified mediator, multiple mediator analysis was conducted by applying linear regression using the SUREG (Zellner's seemingly unrelated regression) command in STATA ${ }^{46}$. Effect sizes and $p$-values were obtained from this model using the assumption-free BOOTSTRAP routine. With these methods, size and proportion of possible indirect (i.e. mediated) effects can be determined. SUREG assumes that the identified mediating effects represent separate pathways, which is not necessarily the case for the selected possible mediators in this study, as they may also lie on the same causal pathway (e.g. social defeat predicting cannabis use), or in the case of social defeat and affective dysregulation, represent a more general affective factor mediating the association between CT and psychosis. Therefore, we further conducted a series of regressions of the identified mediators, deconstructing the multiple mediation model in different steps (CT predicting the mediators [step 1] and the mediators predicting psychosis [step 2]), so that the interrelationships between mediators in the pathway can be intuited. All effect sizes in the linear regression models were standardized, and thus expressed as beta $(\beta)$ coefficients. Continuous psychosis severity was used as the outcome variable in all mediation analyses.

\section{RESULTS}

\section{Prevalence rates}

The prevalence of at least one lifetime psychotic experience validated by reinterview was $6 \%$ (384 out of 6357). The prevalence rate of PD was $0.7 \%$ (43 out of 6357). As expected, 'social defeat' was more prevalent in individuals with increased expression of psychosis (Table 1). In addition, individuals with higher social defeat scores were more likely to be unemployed $(\beta 0.17, \mathrm{Cl} 0.12-0.23, p<0.001)$, were less likely to have received university or higher professional education $(\beta-0.16, \mathrm{Cl}-0.27--0.05, p<0.01)$, and had a lower income ( $\beta$ $0.36, \mathrm{Cl} 0.29-0.43, p<0.001)$. Individuals with higher social defeat scores were less likely to live with a partner $(\beta-0.28, \mathrm{Cl}-0.33--0.23, p<0.001)$, and more likely to be female $(\beta 0.28$, $\mathrm{Cl} 0.23-0.33, p<0.001)$. Individuals with higher $\mathrm{CT}$ scores were also more likely to be female ( $\beta$ 0.19, $\mathrm{Cl} 0.13-0.24, p<0.001$ ), and no evidence for interaction between gender and trauma in terms of social defeat scores was found $(p=0.760)$.

\section{Childhood trauma and psychotic experiences}

CT was significantly associated with the presence of any psychotic experience (OR $1.12, \mathrm{Cl}$ 1.09-1.15, $\mathrm{p}<0.001$ ) and PD (OR 1.10, $\mathrm{Cl} 1.03-1.17, \mathrm{p}=0.003)$. The separate different types of $C T$ were also significantly associated with presence of any psychotic experience (OR [bullying] 1.23, Cl 1.14-1.33, $\mathrm{p}<0.001$; OR [emotional] 1.31, $\mathrm{Cl}$ 1.21-1.41, $\mathrm{p}<0.001$; OR [psychological] 1.28, Cl 1.18-1.38, $\mathrm{p}<0.001$; OR [physical] 1.37, Cl 1.24-1.51, $\mathrm{p}<0.001 ; \mathrm{OR}$ [sexual] 1.39, $\mathrm{Cl} 1.22-1.59, \mathrm{p}<0.001$ ). Only bullying (OR 1.45, $\mathrm{Cl} 1.21-1.72, \mathrm{p}<0.001$ ), emotional abuse (OR 1.23, $\mathrm{Cl} 1.02-1.49, \mathrm{p}=0.034)$ and psychological abuse (OR $1.32, \mathrm{Cl}$ 
1.09-1.61, $\mathrm{p}=0.005$ ) were associated with presence of PD. In addition, CT predicted significantly greater severity of psychotic experiences, both for severity of experiences at the level of the EPP $(\beta 0.15, p<0.001)$ and at the level of PD $(\beta 0.08, p<0.001)$. No evidence for interaction of gender and trauma was found, associated with EPP psychosis severity ( $\beta$ $-0.05, p=0.148)$.

\section{Mediation models}

Higher CT scores were associated with an increase in social defeat scores $(\beta 0.33, p<0.001)$. Higher CT scores were also significantly associated with cannabis use $(\beta 0.13, p<0.001)$ and affective dysregulation $(\beta 0.30, p<0.001)$. Higher social defeat scores predicted higher severity scores for the $\operatorname{EPP}(\beta 0.18, p<0.001)$ and for $\operatorname{PD}(\beta 0.13, p<0.001)$. Higher affective dysregulation scores predicted greater severity of psychotic experiences at the level of the $\operatorname{EPP}(\beta 0.19, p<0.001)$ and of PD $(\beta 0.12, p<0.001)$, as did more cannabis use $\left(\beta_{\text {EPP }} 0.08\right.$, $\left.\mathrm{p}<0.001 ; \beta_{\mathrm{PD}} 0.06, \mathrm{p}<0.001\right)$.

In most of these analyses, no evidence for an interaction with gender was found; in the association of childhood trauma and social defeat $(\beta-0.01, p=0.760)$, the association of CT and affective dysregulation $(\beta-0.02, p=0.540)$, social defeat and EPP severity $(\beta-0.04$, $p=0.204)$, affective dysregulation and EPP severity $(\beta-0.03, p=0.403)$ and cannabis use and EPP severity $(\beta-0.04, p=0.247)$. Childhood trauma did show a significant interaction with gender, associated with cannabis use $(\beta-0.15, p<0.001)$, most likely due to increased cannabis use in males (mean 0.58) compared with females (mean 0.27) (t[11.44], $p<0.001$ ).

In addition, social defeat and affective dysregulation showed evidence for mediation at the level of the EPP and PD, whereas for cannabis use mediation was not clear-cut (Table 2).

Based on these results, social defeat and affective dysregulation were fitted in the SUREG multiple mediator model. The SUREG analysis indicated evidence for mediation by affective dysregulation at the level of the extended psychosis phenotype, but not at the level of psychotic disorder, whereas supportive evidence for mediation was found for social defeat at both levels, albeit borderline significant at the level of the extended psychosis phenotype (Table 3 ).

Additional regression analyses deconstructing the multiple mediation model in two steps (childhood trauma predicting the mediators [step 1] and the mediators predicting psychosis [step 2]) revealed complete mediation by social defeat in the association between CT and affective dysregulation (step 1; Table 4). In step 2, the effects of social defeat on the extended psychosis phenotype were mediated by affective dysregulation, while this was not the case at the level of psychotic disorder, in agreement with the SUREG analysis (Table 4). 
Table 2 NEMESIS-2 baseline assessment. Standardized effect sizes of childhood trauma associated with severity of psychotic experiences at the level of the extended psychosis phenotype, as well as with severity of psychotic experiences in psychotic disorder.

\begin{tabular}{ll}
\hline $\begin{array}{l}\text { Effect size of childhood trauma } \\
\text { psychosis }\end{array}$ & $\begin{array}{l}\text { Possible mediator added to the model ( } \beta \text {; effect size of } \\
\text { possible mediator associated with psychosis) }\end{array}$ \\
\hline
\end{tabular}

Severity of psychotic experiences ${ }^{\mathrm{B}}$ : extended psychosis phenotype

$$
\begin{aligned}
& 0.21 * * * \\
& 0.20 * * * \\
& 0.17 * * * \\
& 0.16 * * *
\end{aligned}
$$

Severity of psychotic experiences ${ }^{\mathrm{A}}$ : psychotic disorder

$$
\begin{aligned}
& 0.11 * * * \\
& 0.10 * * * \\
& 0.07 * * * \\
& 0.08 * * *
\end{aligned}
$$

Cannabis use ${ }^{\mathrm{C}}\left(0.05^{* *}\right)$

Social defeat ${ }^{D}\left(0.13^{* * *}\right)$

Affective dysregulation ${ }^{E}\left(0.14^{* * *}\right)$

\author{
Cannabis use ${ }^{\mathrm{C}}$ (ns) \\ Social defeat ${ }^{D}\left(0.11^{* * *}\right)$ \\ Affective dysregulation ${ }^{E}\left(0.10^{* * *}\right)$
}

Note: Possible mediator variables (cannabis use, social defeat and affective dysregulation) were added separately to the model. Possible mediators in bold and italic diminish the effect size of childhood trauma associated with psychosis with more than $10 \%$ (left column), and show significant associations with psychosis. All effect sizes were adjusted for age and sex. $* * * p<0.001$, $* * p<0.01$, ns: not significant.

A Summed score of five types of childhood trauma before age 16 years, each on a scale of 0 (never) to 5 (very often)

B Summed score of distress, frequency and impact, each on scale of 1 (seldom or no) to 4 (almost always or much)

c Scale of 0 (never) to 6 (every day). Adding cannabis use to the model yielded minimal change in the effect size of childhood trauma, and was therefore added as a confounder in further analyses, not as a possible mediator.

D Summed score of 7 affective symptoms indexing social defeat

E Summed score of 21 general affective dysregulation symptoms

Table 3 NEMESIS-2 baseline assessment; SUREG mediation results. All effect sizes were adjusted for age, cannabis use and sex.

\begin{tabular}{llll}
\hline & $\begin{array}{l}\text { Indirect effect } \\
\text { size ( } \beta \text { ) }\end{array}$ & p-value & $\begin{array}{c}\text { Proportion of } \\
\text { effect (\%) }\end{array}$ \\
\hline Severity of psychotic experiences: extended psychosis phenotype & & \\
Affective dysregulation & 0.04 & 0.002 & 30.7 \\
Social defeat & 0.03 & 0.081 & 80.4 \\
Total indirect effect & 0.07 & 0.000 & 19.6 \\
Direct effect of childhood trauma & & & n.a. \\
Severity of psychotic experiences: psychotic disorder & & 0.557 & 86.6 \\
Affective dysregulation & 0.01 & 0.004 & 86.6 \\
Social defeat & 0.04 & 0.000 & 13.4 \\
Total indirect effect & 0.04 & & \\
Direct effect of childhood trauma & & & \\
\hline
\end{tabular}

Note: Proportion of effect (right column) indicates the percentage of the total effect in the association between childhood trauma and psychosis severity that is mediated. For the extended psychosis phenotype, the direct effect of childhood trauma was $19.6 \%$ (thus about $20 \%$ of the change in psychosis outcome was due to increased frequency of experienced childhood trauma), the indirect effect of affective dysregulation was $49.7 \%$ and of social defeat $30.7 \%$. For psychotic disorder, the direct effect of childhood trauma was $13.4 \%$, and the indirect effect of social defeat was $86.6 \%$. n.a., not applicable. 
Table 4 NEMESIS-2 baseline assessment, regression mediation analysis, first and second step.

\begin{tabular}{lllll}
\hline Outcome & Predictor & $\boldsymbol{\beta}$ & p-value & Mediation \\
\hline Step 1 & & & & \\
Affective dysregulation & Childhood trauma & 0.29 & 0.000 & \\
Social defeat & Childhood trauma & 0.32 & 0.000 & \\
Affective dysregulation & Childhood trauma & 0.01 & 0.171 & Complete mediation by \\
& Social defeat & 0.88 & 0.000 & social defeat \\
Step 2: Extended psychosis phenotype & Affective dysregulation & 0.18 & 0.000 & \\
Severity psychotic experiences & Social defeat & 0.17 & 0.000 & \\
Severity psychotic experiences & Social defeat & 0.04 & 0.172 & Partial mediation by \\
Severity psychotic experiences & Affective dysregulation & 0.15 & 0.000 & affective dysregulation \\
& & & & \\
Step 2: Psychotic disorder & & 0.11 & 0.000 & \\
Severity psychotic experiences & Affective dysregulation & 0.13 & 0.000 & \\
Severity psychotic experiences & Social defeat & 0.11 & 0.000 & No mediation by affective \\
Severity psychotic experiences & Social defeat & 0.02 & 0.330 & dysregulation \\
& Affective dysregulation & 0.00 &
\end{tabular}

Step one: Effects of childhood trauma on social defeat and affective dysregulation.

Each row displays the outcome and predictor(s) that were added to the model, with corresponding standardized effect sizes. The first two rows were two separate univariable analyses and the last row was a multivariable analysis (with childhood trauma and social defeat as predictors).

Step two: Effects of social defeat and affective dysregulation on psychosis severity (in context of EPP or PD).

All effect sizes were adjusted for age, sex and cannabis use. 


\section{DISCUSSION}

The present study found an association between the experience of childhood trauma and the expression of psychosis, both at the level of psychotic experiences in the population and at the level of PD. These findings replicate earlier studies on the association between childhood trauma and psychosis ${ }^{6,7,10-12}$. There was also evidence for a dose-response relationship: increased frequency of childhood adverse experiences was associated with increased severity of psychotic experiences.

In addition, we present evidence that social defeat acts as a possible underlying mechanism relating childhood trauma to psychosis. The mediating role of social defeat is in line with the hypothesis put forward by Selten and Cantor-Graae ${ }^{23}$, that experiences that lead individuals to feel rejected by their immediate social environment (i.e. their peers or direct family) may increase risk for psychosis by causing feelings of outsider status and not fitting in. There is replicated evidence that being the exception in relation to the wider social environment increases the risk for psychotic disorder ${ }^{47,48}$. A more recent study of all Swedes born between 1972 and 1977 found that characteristics that define individuals as being different from most of the people in their local environment were associated with risk of schizophrenia ${ }^{49}$. For example, coming from another ethnic background was associated with a higher risk of psychosis, but much more so if non-native individuals were in schools with a high percentage of native Swedes, suggesting that the experience of 'not fitting-in' may be an important factor in the association between early social risk and schizophrenia. Importantly, the present results show the largest OR for being bullied (1.45 for each one-point increase in frequency of bullying), suggesting the influence of peers may be decisive in bringing about feelings of rejection.

In addition to social defeat, affective dysregulation was an important mediator linking childhood trauma and psychosis. The results of this study thus suggest a developmental pathway, in which childhood trauma may lead to feelings of social defeat, which in turn may result in affective dysregulation and psychotic symptoms (partially mediated by affective dysregulation) in the general population (Figure 1a). At the highest levels of psychosis expression (psychotic disorder), social defeat may directly and exclusively mediate the association between childhood trauma and psychosis (Figure 1b). The divergence of these patterns of mediation can be understood from the difference between the extended psychosis phenotype and the phenotype of psychotic disorder. Psychotic experiences as part of the extended psychosis phenotype in the general population are distributed across all diagnostic categories of mental disorder (psychotic and non-psychotic) and are particularly prevalent in disorders characterised by affective dysregulation such as disorders of anxiety and depression ${ }^{27}$, which are also associated with childhood trauma ${ }^{50}$. Mediation by affective dysregulation therefore may be more common if psychosis occurs in the context of non-psychotic outcomes, whereas social defeat may play a more prominent role in the more narrow outcome of psychotic disorder. 


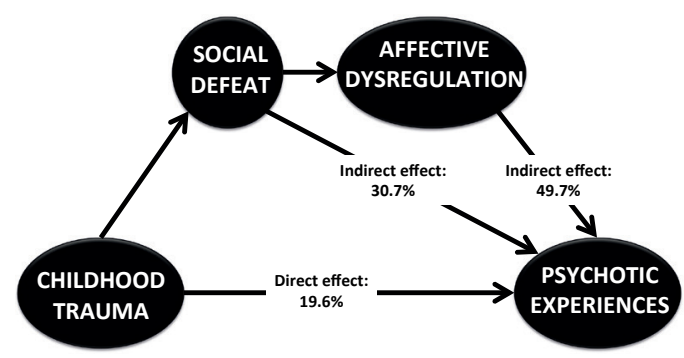

Figure 1a Childhood trauma leads to social defeat, which in turn leads to psychotic experiences in the general population (extended psychosis phenotype), with mediation by affective dysregulation

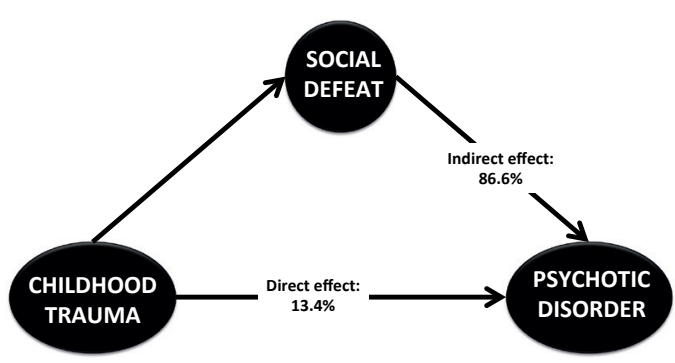

Figure $1 b$ Effects of childhood trauma on the development of psychotic disorder is mediated by social defeat.

The present study underscores the importance of incorporating trauma history in standard mental health care, contrary to current medical practice ${ }^{51}$. Furthermore, efforts to prevent childhood trauma may have the potential to reduce incidence rates of psychosis, as indicated by the population attributable risk of $33 \%$ reported by Varese and colleagues ${ }^{14}$.

These findings should be interpreted in the light of several strengths and limitations. In terms of strengths, the present study has the advantage of a high number of participants representative of the Dutch population, with clinical validation of reported psychotic experiences, which is especially important in general population studies ${ }^{16,17}$.

The most obvious limitation of the current study is its cross-sectional nature, precluding conclusions of causality (social defeat causing psychosis). Further follow-up is required for this type of inference. Another limitation, although difficult to avoid, is the retrospective nature of the information on childhood trauma. Recall bias may have influenced these data; however other studies have found that recall of childhood trauma is reliable, even in patients with psychotic disorder ${ }^{52}$. Lastly, the operationalization of 'social defeat' was done at face value. However, the items were chosen a priori based on theoretical considerations ${ }^{23}$ and showed robust evidence for consistency using factor analysis. Even though it must be acknowledged that these items overlap with the cognitive symptoms of depression, highly similar items have previously been described as specific 'defeat' items for a validated scale in social rank theory research ${ }^{53}$. Moreover, the association between childhood trauma and affective dysregulation was completely mediated by social defeat, supporting the claim that they represent separate constructs rather than a single, general affective factor.

These social defeat items were assessed in the context of more stable personality traits or general presence of feelings, with some overlap with 'Early Maladaptive Schemas' from the Young Schema Questionnaire ${ }^{54}$. The epidemiological design of the present study does not allow for the assessment of feelings of 'defeat' in the context of an actual social setting. A recent study has attempted to disentangle the differences between the personal interpretation of social situations on the one hand, and actual social interactions as experienced by others, by comparing self-reports and peer-reports of peer victimization ${ }^{55}$. 
In this study, it was found that not peer-reports, but the subjective (personal) reports of victimization were associated with presence of psychotic experiences. This may indicate that these internal interpretations may be the key components that are associated with psychosis, in agreement with findings from the present study.

Future research focused on the association of social defeat and psychosis should incorporate more dynamic approaches, such as momentary assessment strategies ${ }^{56}$, which are able to measure feelings of defeat in response to certain social situations, in order to better capture relevant underlying mechanisms.

\section{Funding}

This work was supported by the Ministry of Health, Welfare and Sport (grant number 310253); with supplement support from the Netherlands Organization for Health Research and Development (ZonMw) and the Genetic Risk and Outcome of Psychosis (GROUP) investigators. Supported by the European Community's Seventh Framework Program under grant agreement No. HEALTH-F2-2009-241909 (Project EU-GEI).

\section{Acknowledgements}

The Netherlands Mental Health Survey and Incidence Study-2 (NEMESIS-2) is conducted by the Netherlands Institute of Mental Health and Addiction (Trimbos Institute) in Utrecht. The reinterviews are conducted by the University of Maastricht, department of Psychiatry and Psychology. 


\section{REFERENCES}

1. Read J, van Os J, Morrison AP, Ross CA. Childhood trauma, psychosis and schizophrenia: a literature review with theoretical and clinical implications. Acta Psychiat Scand 2005;112:330-350.

2. Bendall $S$, Jackson $H J$, Hulbert CA. Childhood trauma and psychosis: Review of the evidence and directions for psychological interventions. Australian Psychol 2010;45(4):299-306.

3. Morgan C, Fisher H. Environmental factors in schizophrenia: childhood trauma - a critical review. Schizophr Bull 2007;33(1):3-10.

4. Cutajar MC, Mullen PE, Ogloff JRP, Thomas SD, Wells DL, Spataro J. Schizophrenia and other psychotic disorders in a cohort of sexually abused children. Arch Gen Psychiat 2010;67(11):1114-1119.

5. Fisher $\mathrm{HL}$, Jones $\mathrm{PB}$, Fearon $\mathrm{P}$, et al. The varying impact of type, timing and frequency of exposure to childhood adversity on its association with adult psychotic disorder. Psychol Med 2010;40:1967-1978.

6. Arseneault $L$, Cannon $M$, Fisher $\mathrm{H}$, Polanczyk G, Moffitt TE, Caspi A. Childhood trauma and children's emerging psychotic symptoms: a genetically sensitive longitudinal cohort study. Am J Psychiatry 2011;168:65-72.

7. Whitfield $\mathrm{CL}$, Dube SR, Felitti VJ, Anda RF. Adverse childhood experiences and hallucinations. Child $\mathrm{Ab} \mathrm{Neg}$ 2005;29:797-810.
8. Wigman JTW, Van Winkel R, Ormel J, Verhulst FC, Van Os J, Vollebergh WAM. Early trauma and familial risk in the development of the extended psychosis phenotype in adolescence. Acta Psychiat Scand 2012;126(4):266-273.

9. Steel C, Marzillier S, Fearon P, Ruddle A. Childhood abuse and schizotypal personality. Soc Psych Psych Epidem 2009;44:917-923.

10. Heins $M$, Simons $C$, Lataster $T$, et al. Childhood trauma and psychosis: A case-control and case-sibling comparison across different levels of genetic liability, psychopathology, and type of trauma. Am J Psychiatry 2011;168(12):1286-1294.

11. Lataster T, Van Os J, Drukker M, Henquet C, Feron F, Gunther N, Myin-Germeys I. Childhood victimisation and developmental expression of non-clinical delusional ideation and hallucinatory experiences. Soc Psych Psych Epidem 2006;41:423-428.

12. Janssen I, Krabbendam L, Bak M, Hanssen M, Vollebergh W, De Graaf R, Van Os J. Childhood abuse as a risk factor for psychotic experiences. Acta Psychiat Scand 2004;109:38-45.

13. Ucok A, Bıkmaz S. The effects of childhood trauma in patients with first-episode schizophrenia. Acta Psychiat Scand 2007;116:371-377. 
14. Varese F, Smeets F, Drukker M, et al. Childhood adversities increase the risk of psychosis: a meta-analysis of patient-control, prospective- and cross-sectional cohort studies. Schizophr Bull 2012;38(4):661-671.

15. Bebbington $P$, Jonas $S$, Kuipers $E$, et al. Childhood sexual abuse and psychosis: data from a cross-sectional national psychiatric survey in England. Br J Psychiatry 2011:1-9.

16. Linscott RJ, Van Os J. Systematic reviews of categorical versus continuum models in psychosis: evidence for discontinuous subpopulations underlying a psychometric continuum. Implications for DSM-V, DSM-VI, and DSM-VII. Ann Rev Clin Psychol 2010;6:391-419.

17. van Nierop M, Van Os J, Gunther N, et al. Phenotypically continuous with clinical psychosis, discontinuous in need for care: Evidence for an extended psychosis phenotype. Schizophr Bull 2012;38(2):231-238.

18. Bentall R, Fernyhough C. Social predictors of psychotic experiences: specificity and psychological mechanisms. Schizophr Bull 2008;34:1009-1011.

19. Read J, Bentall R, Fosse R. Time to abandon the bio-bio-bio model of psychosis: exploring the epigenetics and psychological mechanisms by which adverse life events lead to psychotic symptoms. Epidem Psich Soc 2009;18(4):299-310.

20. Krishnan V, Han MH, Graham DL, et al. Molecular adaptations underlying susceptibility and resistance to social defeat in brain reward regions. Cell 2007;131:391-404.
21. Morgan D, Grant K, Gage D, et al. Social dominance in monkeys: dopamine $D_{2}$ receptors and cocaine self-administration. Nature Neurosc 2002;5(2):169-174.

22. Cantor-Graae E, Selten JP. Schizophrenia and migration: a meta-analysis and review. Am J Psychiatry 2005;162:12-24.

23. Selten JP, Cantor-Graae E. Social defeat: risk factor for schizophrenia? B J Psychiatry 2005;187:101-102.

24. Stowkowy J, Addington J. Maladaptive schemas as a mediator between social defeat and positive symptoms in young people at clinical high risk for psychosis. Early Int Psychiatry 2012;6:87-90.

25. Taylor PJ, Gooding P, Wood AM, Johnson J, Pratt D, Tarrier N. Defeat and entrapment in schizophrenia: the relationship with suicidal ideation and positive psychotic symptoms. Psychiat Res 2010;178:244-248.

26. van Winkel R, Stefanis NC, MyinGermeys I. Psychosocial stress and psychosis. A review of the neurobiological mechanisms and the evidence for gene-stress interaction. Schizophr Bull 2008;34(6):1095-1105.

27. Wigman JTW, van Nierop $M$, Vollebergh W, Lieb R, Beesdo-Baum K, Wittchen HU, Van Os J. Evidence that psychotic symptoms are prevalent in disorders of anxiety and depression, impacting on illness onset, risk, and severity - implications for diagnosis and ultra-high risk research. Schizophr Bull 2012;38(2):247-257. 
28. Van Rossum I, Dominguez MD, Lieb R, Wittchen HU, Van Os J. Affective dysregulation and reality distortion: a 10-year prospective study of their association and clinical relevance. Schizophr Bull 2011;37(3):561-571.

29. Nanni V, Uher R, Danese A. Childhood maltreatment predicts unfavorable course of illness and treatment outcome in depression: a meta-analysis. Am J Psychiatry 2012;169:141-151.

30. Oshri A, Rogosch FA, Burnette ML, Cicchetti D. Developmental pathways to adolescent cannabis abuse and dependece: child maltreatment, emerging personality, and internalizing versus externalizing psychopathology. Psychol Add Beh 2011;25(4):634-644.

31. Moore THM, Zammit $S$, LingfordHughes A, Barnes TRE, Jones PB, Burke M, Lewis $G$. Cannabis use and risk of psychotic or affective mental health outcomes: a systematic review. Lancet 2007;370(9584):319-328.

32. Bijl RV, van Zessen $G$, Ravelli $A$, de Rijk C, Langendoen Y. The Netherlands Mental Health Survey and Incidence Study (NEMESIS): Objectives and design Soc Psych Psych Epidem 1998;33:581-586.

33. De Graaf $R$, ten Have $M$, van Dorsselaer S. The Netherlands Mental Health Survey and Incidence study-2 (NEMESIS-2): design and methods. Int $J$ Meth Psych Res 2010;19(3):125-141.

34. Alonso J, Angermeyer M, Bernert S, et al. Sampling and methods of the European Study of the Epidemiology of Mental Disorders (ESEMeD) project. Acta Psychiat Scand 2004;109(Suppl. 420):8-20.
35. De Graaf R, Ormel J, Ten Have M, Burger $\mathrm{H}$, Buist-Bouwman M. Mental disorders and service use in the Netherlands. Results from the European Study of the Epidemiology of Mental Disorders (ESEMeD). The WHO World Mental Health Surveys: Global Perspectives on the Epidemiology of Mental Disorders. Kessler, R.C., Üstün, T.B. ed: Cambridge University Press; 2008:388-405.

36. De Graaf R, Ten Have M, van Gool C, Van Dorsselaer S. Prevalence of mental disorders and trends from 1996 to 2009. Results from the Netherlands Mental Health Survey and Incidence Study-2. Soc Psych Psych Epidem 2012;47(2):203-213.

37. Andrew G, Peters L. The psychometric properties of the Composite International Diagnostic Interview. Soc Psych Psych Epidem 1998;33:80-88.

38. Helzer JE, Robins LN, McEvoy LT, Spitznagel EL, Stoltzman RK, Farmer A, Brockington IF. Physician reexamination of lay-interviewed cases in the general population. Arch Gen Psychiat 1985;42(7):657-666.

39. Spitzer RL, Williams JB, Gibbon M, First MB. The Structured Clinical Interview for DSM-III-R (SCID). I: History, rationale, and description. Arch Gen Psychiat 1992;49:624-629.

40. Van Os J, Linscott RJ, Myin-Germeys I, Delespaul P, Krabbendam L. A systematic review and meta-analysis of the psychosis continuum: evidence for a psychosis-proneness-persistence-impairment model of psychotic disorder. Psychol Med 2009;39:179-195. 
41. Statacorp. Stata Statistical Software:

Release 11. College Station, TX:

StataCorp LP. 2009.

42. van Winkel $R$, Genetic Risk and Outcome of Psychosis (GROUP) investigators. Family-based analysis of genetic variation underlying psychosis-inducing effects of cannabis. Arch Gen Psychiat 2011;68(2):148-157.

43. Krabbendam L, Myin-Germeys I, Hanssen M, De Graaf R, Vollebergh W, Bak M, van Os J. Development of depressed mood predicts onset of psychotic disorder in individuals who report hallucinatory experiences. $B \mathrm{~J}$ Clin Psychol 2005;44:113-125.

44. Fisher H, Morgan C, Dazzan P, et al. Gender differences in the association between childhood abuse and psychosis. B J Psychiatry 2009;194(4):319-325.

45. Hovens JGFM, Wiersma JE, Spinhoven P, Penninx BWJH, Zitman FG. Impact of childhood life events on the course of depressive and anxiety disorders. Acta Psychiatr Scand 2012;DOI: 10.1111/j.1600-0447.2011.01828.x.

46. Zellner $A$. An efficient method of estimating seemingly unrelated regression equations and tests of aggregation bias. J Am Stat Assoc 1962;57:500-509.

47. Veling $W$, Susser E, van Os J, Mackenbach JP, Selten JP, Hoek HW. Ethnic density of neighborhoods and incidence of psychotic disorders among immigrants. A J Psychiatry 2008;165:66-73.

48. Boydell J, Van Os J, McKenzie K, Allardyce J, Goel R, McCreadie RG, Murray RM. Incidence of schizophrenia in ethnic minorities in London: ecological study into interactions with environment. $B$ M J 2001;323:1-4.

49. Zammit $S$, Lewis $G$, Rasbach J, Dalman C, Gustafsson JE, Allebeck P. Individuals, schools and neighborhood. Arch Gen Psychiat 2010;67(9):914-922.

50. Matheson SL, Shepherd AM, Pinchbeck RM, Laurens KR, Carr VJ. Childhood adversity in schizophrenia: a systematic meta-analysis. Psychol Med 2012;43(2):225-238.

51. Read J, Hammersley P, Rudegeair T. Why, when and how to ask about childhood abuse. Adv Psychiat Treat 2007;13:101-110.

52. Fisher HL, Craig TK, Fearon $\mathrm{P}$, et al. Reliability and comparability of psychosis patients' retrospective reports of childhood abuse. Schizophr Bull 2011;37(3):546-553.

53. Gilbert $P$, Allan $\mathrm{S}$. The role of defeat and entrapment (arrested flight) in depression: an exploration of an evolutionary view. Psychol Med 1998;28:585-598.

54. Young J. Cognitive therapy for personality disorders: a schema-focused approach. 2 ed. Sarasota, FL: Professional Resource Press; 1994.

55. Gromann PM, Goossens FA, Olthof T, Pronk J, Krabbendam L. Selfperception but not peer reputation of bullying victimization is associated with non-clinical psychotic experiences in adolescents. Psychol Med 2013;43:781-787.

56. Myin-Germeys I, Oorschot M, Collip D, Lataster J, Delespaul P, van Os J. Eperience sampling research in psychopathology: opening the black box of daily life. Psychol Med 2009;39:1533-1547. 


\title{
CHAPTER 7
}

\section{Childhood Trauma and Polygenic Risk for Schizophrenia: No Evidence for Gene-Environment Correlation or Gene-Environment Interaction in a Family-Based Sample}

\author{
Martine van Nierop ${ }^{A^{*}}$ \\ Ruud van Winkel $\mathrm{I}^{\mathrm{AB}}$ \\ Wolfgang Viechtbauer ${ }^{A}$ \\ Marjan Drukker ${ }^{A}$ \\ Bart P. F. Rutten ${ }^{A}$ \\ Christiaan Vinckers ${ }^{\mathrm{C}}$ \\ Alexander Richards ${ }^{\mathrm{D}}$ \\ Mike Owens ${ }^{\mathrm{D}}$ \\ Peter Holmans ${ }^{D}$ \\ Michael O'Donovan ${ }^{\text {D }}$ \\ The Psychiatric Genomic Consortium \\ Genetic Risk and OUtcome of Psychosis (GROUP) Investigators ${ }^{\S}$.
}

\author{
* These authors contributed equally to this work \\ $\S$ The GROUP Investigators are: \\ Richard Bruggeman ${ }^{\mathrm{E}}$ \\ Wiepke Cahn ${ }^{c}$ \\ Lieuwe de Haan ${ }^{\mathrm{F}}$ \\ René S. Kahn ${ }^{\mathrm{C}}$ \\ Carin J. Meijer ${ }^{\mathrm{F}}$ \\ Inez Myin-Germeys ${ }^{A}$ \\ Jim van $\mathrm{Os}^{\mathrm{AG}}$ \\ Durk Wiersma ${ }^{\mathrm{E}}$
}

A Maastricht University Medical Centre, Dept. of Psychiatry and Psychology, School for Mental Health and Neuroscience, EURON, Maastricht, The Netherlands

B University Psychiatric Center Katholieke Universiteit Leuven, campus Kortenberg, Leuvensesteenweg, Kortenberg, Belgium C University Medical Center Utrecht, Department of Psychiatry, Rudolf Magnus Institute of Neuroscience, The Netherlands D Cardiff University School of Medicine, Institute of Psychological Medicine and Clinical Neuroscience, Maindy Road,

Cathays, Cardiff, UK

E University Medical Center Groningen, Department of Psychiatry, University of Groningen, The Netherlands

F Academic Medical Centre University of Amsterdam, Department of Psychiatry, Amsterdam The Netherlands

G King's College London, King's Health Partners, Department of Psychosis Studies, Institute of Psychiatry, London, United Kingdom

Submitted 


\section{ABSTRACT}

\section{Background}

Meta-analytic evidence ties childhood trauma to an increased risk for psychosis, but it is unknown whether this link is causal or that genetic risk for schizophrenia increases the risk for victimization (i.e., gene-environment correlation).

\section{Methods}

Data on childhood trauma and polygenic risk scores for schizophrenia were available for 439 white European patients with schizophrenia, 476 of their non-psychotic siblings, and 322 white European controls.

\section{Results}

Associations between childhood trauma and psychosis were confirmed the level of patient status (case-control: Odds Ratio [OR] 5.2, 95\% Cl 3.3-8.0, p<0.001; case-sibling: OR $3.1,95 \% \mathrm{Cl} 2.2-4.2, \mathrm{p}<0.001$ ), as well as at the level of schizotypy in controls ( $\mathrm{B} 0.18,95 \%$ $\mathrm{Cl} 0.09-0.28, \mathrm{p}<0.001$ ) and non-psychotic siblings (B $0.22,95 \% \mathrm{Cl} 0.16-0.28, \mathrm{p}<0.001$ ). Polygenic risk for schizophrenia did not predict exposure to childhood trauma in controls, non-psychotic siblings, or patients, or in the combined sample. Moreover, associations between childhood trauma and psychosis remained comparable after controlling for polygenic risk (case-control OR 6.0, 95\% Cl 3.5-10.4, p<0.001; case-sibling OR 3.0, 95\% Cl $2.0-4.5, p<0.001$ ). No evidence for statistical interaction between polygenic risk and childhood trauma was found.

\section{Conclusion}

Gene-environment correlation is unlikely to explain the association between childhood trauma and psychosis, supporting a causal contribution. The timing, nature, and duration of traumatic experiences may be more important than genetic background in determining which individuals develop psychotic experiences following trauma. 


\section{INTRODUCTION}

The association between childhood traumatic experiences and psychosis has been clearly established in recent meta-analytic work ${ }^{1}$. However, the contribution of childhood trauma as a causal risk factor for psychosis is much debated, as plausible alternative explanations for the link between childhood trauma and psychosis, such as reverse causation and geneenvironment correlation, have not been sufficiently addressed.

Reverse causation denotes the situation where, instead of an environmental factor causing later psychotic experiences, the expression of psychotic experiences increases the risk of exposure to an environmental factor ${ }^{2}$. In the context of childhood trauma, it is quite plausible that an individual with psychotic experiences may be more likely to be victimized or bullied. A recent paper by Kelleher and colleagues indeed reported that children aged 13 to 16 who reported psychotic experiences were more likely to experience adversity later in life ${ }^{3}$. However, the reverse was equally true, in that childhood traumatic experiences were predictive of later psychotic experiences. Importantly, cessation of the adverse experience resulted in a decrease of psychotic experiences, in agreement with a possible causal explanation. Together with a number of prospective studies showing that early traumatic experiences are predictive of later psychotic disorder ${ }^{4,5}$, it therefore seems unlikely that reverse causation can entirely explain the link between childhood trauma and psychosis.

The possibility that a person at genetic risk for psychosis is more likely to experience adversities in childhood, however, so far has not been clearly addressed. Gene-environment correlation can take the form of 'genetic confounding', in which case the environmental factor is non-causal and merely a correlate of underlying genetic risk (Figure 1a), or it can take the form of 'environmental mediation of genetic risk' (genetic risk leads to childhood trauma, which further increases the risk for psychosis compared to genetic risk alone) (Figure 1b). One study reported higher rates of childhood trauma in siblings of patients with schizophrenia ${ }^{6}$, compared with controls, possibly compatible with gene-environment correlation (Figure 1a).

Figure 1 Alternative explanations for findings of associations between childhood trauma and psychosis

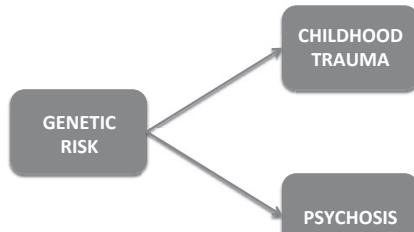

Figure 1a: Genetic confounding (non-causal); childhood trauma is merely a correlate of underlying genetic risk

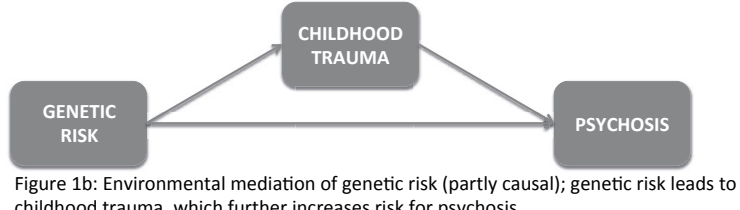

Figure 1b: Environmental mediation of genetic risk (partly causal); genetic risk leads to childhood trauma, which further increases risk for psychosis 
Recent developments in the field of psychiatric genetics have made it possible to partially quantify genetic risk for schizophrenia, using so-called polygenic risk scores ${ }^{7}$. These scores consist of the weighted sum of top ranking (assessed by their $p$-values) markers in a training set, usually a large, genome-wide association study ${ }^{8}$. Studies have shown that liberal thresholds for inclusion ( $p$-values $<.5$ ) are able to capture genetic risk most adequately,, thus providing a useful instrument to quantify genetic risk for schizophrenia.

The present study aimed to examine (1) whether genetic risk for schizophrenia predicts exposure to childhood traumatic events (gene-environment correlation), (2) whether the association between childhood trauma remains significant after controlling for genetic risk, and (3) examine the possibility of interaction between polygenic risk for schizophrenia and childhood trauma on the development of psychosis.

\section{METHODS}

\section{Sample}

The sample of the present study consists of 611 white European non-affective psychotic disorder patients, 591 of their non-psychotic siblings, and 381 white European controls. Of these, 439 patients, 476 siblings, and 322 controls completed the Childhood Trauma Questionnaire ${ }^{10}$ (CTQ; see below: measures). The siblings and controls that completed the CTQ did not differ in schizotypy scores (see below: measures) compared with siblings and controls that did not complete the CTQ. Controls without CTQ scores were older (mean nocTQ $31.7 \mathrm{yrs}$, mean $^{\text {стQ }} 26.6 \mathrm{yrs}, \mathrm{t}=3.51, \mathrm{p}<0.001$ ), more often male (mean ${ }^{\text {nocTQ }} 0.41$, mean ${ }^{\text {стQ }}$ $0.59, \mathrm{t}=2.58, \mathrm{p}=0.010$ ). Siblings and patients who did not complete the CTQ did not differ in terms of age and sex compared with siblings and patients who did. Patients, siblings and controls that did not complete the CTQ did not differ in terms of polygenic risk scores from participants with completed CTQs (patients $t=-1.00, p=0.318$, siblings $t=0.55$, $p=0.583$, controls $t=-1.55, p=0.123$ ). Of the participants with completed CTQ's, 472 siblings and 316 controls participated in the Structured Interview for Schizotypy-Revised ${ }^{11}$ (SIS-R; see below: measures). Subjects who did not participate in the SIS-R interview did not differ in terms of age, gender, or polygenic risk scores, compared with subjects who were available for SIS-R assessment. Table 1 provides an overview of all participants included in the analyses. 
Table 1 Baseline measurement and demographics of GROUP

\begin{tabular}{llll}
\hline & $\begin{array}{l}\text { Patients }(\mathbf{n}=439) \\
\text { Mean (SD) }\end{array}$ & $\begin{array}{l}\text { Unaffected siblings (n=476) } \\
\text { Mean (SD) }\end{array}$ & $\begin{array}{l}\text { Healthy controls }(\mathbf{n}=322) \\
\text { Mean (SD) }\end{array}$ \\
\hline Age (at baseline) & $27.3(7.3)$ & $27.2(7.8)$ & $31.7(10.5)$ \\
Childhood trauma $^{\mathrm{A}}$ & $1.6(0.5)$ & $1.4(0.4)$ & $1.3(0.3)$ \\
Polygenic risk score $^{\mathrm{B}}$ & $0.04(0.70)$ & $-0.27(0.40)$ & $-0.36(0.86)$ \\
SIS-R total schizotypy score $^{\mathrm{c}}$ & $\mathrm{n} . \mathrm{a}$. & $0.3(0.3)$ & $0.3(0.3)$ \\
& $\mathrm{N}(\%)$ & $\mathrm{N}(\%)$ & $\mathrm{N}(\%)$ \\
Gender (male) & $320(73)$ & $226(48)$ & $133(41)$ \\
\hline
\end{tabular}

A Childhood trauma is an average score of 25 items each scored on a 5-point Likert scale, range 1-3.8

B Polygenic risk score ranging from -3.12 to 2.88 , constructed using the data release from the Schizophrenia Working Group of the Psychiatric Genomic Consortium meta-analysis as a discovery set ${ }^{12}$

C SIS-R total score is an average all schizotypy subscales, range 0-1.7

Inclusion criteria were: (i) age range 16 to 50 years and (ii) good command of Dutch language. For patients, an additional inclusion criterion was the presence of a clinical diagnosis of non-affective psychotic disorder. Diagnosis was based on the Diagnostic and Statistical Manual of Mental Disorder-IV (DSM-IV) criteria $^{13}$, assessed with the Comprehensive Assessment of Symptoms and History (CASH) interview ${ }^{14}$ or Schedules for Clinical Assessment for Neuropsychiatry (SCAN 2.1) ${ }^{15}$. Control subject status was confirmed by using the Family Interview for Genetic studies ${ }^{16}$ with the control as informant, to establish absence of first degree relatives with a psychotic disorder. Participants were excluded if their estimated level of intelligence was below 70 (as assessed with the short form of the Wechsler Adult Intelligence Scale [WAIS] - III ${ }^{17}$. For a more detailed description of recruitment of the participants, see Korver et al. ${ }^{18}$. All questionnaires were administered by trained interviewers, diagnoses were confirmed by psychiatrists.

The study was approved by the standing ethics committee (Medisch Ethische Toetsingscommissie, UMC Utrecht), and all the subjects gave written informed consent in accordance with the committee's guidelines.

\section{Measures}

Genotyping and polygenic risk scores

Subjects were genotyped at the University of California, Los Angeles (UCLA) Neurosciences Genomic Core (UNGC) using the illumina HumanHap550 beadchip, or at the Welcome Trust Case-Control Consortium facilities using the Affymetrix SNP array 6.0. An extensive quality control (QC) protocol was carried out, the procedure is described in full detail elsewhere ${ }^{12}$. Briefly, SNPs were included if the missing rate was $<.02$, the SNP frequency difference to HapMap was $<.15$, the difference missing rate per SNP between cases and controls $<.02$, and Hardy-Weinberg Equilibrium was not violated in controls $\left(p<10^{-6}\right)$. Individuals were included if the missing rate was $<.02$. Polygenic scores were constructed using the data release from the Schizophrenia Working Group of the Psychiatric Genomics Consortium 
meta-analysis as a discovery set (after excluding samples that overlap with the sample set presented in this article) ${ }^{12}$. These SNPs were linkage disequilibrium (LD) pruned using the 'clumping' procedure implemented in PLINK ${ }^{19}$, which retains those SNPs most associated with schizophrenia in the discovery set from each LD block (maximum $\mathrm{r} 2=0.25$, window $=250 \mathrm{~kb}$ ). Additive polygenic scores, weighted by the log odds ratio of each SNP in the discovery set, were then calculated for the samples presented here using PLINK, using a $p$-value threshold of $p<.1$, as the polygenic scores at this threshold explained most of the variance compared to other cut-offs in the discovery set ${ }^{12}$.

\section{Childhood trauma}

Childhood trauma was assessed with the Dutch version of the Childhood Trauma Questionnaire (CTQ-SF ${ }^{10}$ ), a 25-item self-report questionnaire rated on a scale of 1 (never true) to 5 (very often true). The CTQ measures physical abuse, physical neglect, sexual abuse, emotional abuse, and emotional neglect, occurring before the age of 16 . An average score of all items was used for an overall trauma score (possible range 1-5).

\section{Schizotypy}

Subclinical psychosis, or schizotypy, was assessed with the Structured Interview for Schizotypy-Revised (SIS-R ${ }^{11}$ ) in the sibling and control groups. The SIS-R is a semi-structured interview containing 20 schizotypal symptoms and 11 schizotypal signs, rated on a fourpoint scale. Symptoms are defined as verbal responses to standardized questions concerning, for example, magical ideation, illusions, and referential thinking. Signs refer to behaviors that are rated by the interviewers, for example, goal directedness of thinking and flatness of affect. Questions and rating procedures are standardized. A total schizotypy score was calculated by assessing the mean of all schizotypal symptoms or signs ${ }^{11}$ (possible range 0-3).

\section{Cannabis use}

Cannabis use has been shown to be associated with childhood trauma and psychotic outcomes, and thus was added as a potential confounder in some of the analyses ${ }^{20,21}$ (see below: analyses). Cannabis use was assessed with the Composite International Diagnostic Interview ${ }^{22}$. Consistent with previous research in this sample ${ }^{23}$, the cannabis pattern of use during the lifetime period of heaviest use was considered most informative and used for analysis: none (0), less than weekly (1), weekly (2), and daily (3). 


\section{Analyses}

\section{Childhood trauma, polygenic risk, and psychosis}

In order to assess associations of childhood trauma with schizophrenia, logistic regression in a case-control and case-sibling design was used, while accounting for clustering of subjects within families by using robust standard errors.

Associations of childhood trauma with total schizotypy scores in the siblings and controls were assessed with two separate censored regression analyses ${ }^{24}$ (using the TOBIT command in Stata), while accounting for clustering within families (using cluster-robust estimates of the standard errors of the model coefficients using the Huber-White method). Tobit regression models allow for the estimation of the relationship between one or more predictor variables and some outcome variable of interest when there is either left and/ or right censoring in the outcome variable ${ }^{24}$. In particular, the model coefficients indicate how unit changes in the predictor variables are related to changes on a latent continuum, which is only observed in censored form via the outcome variable. Censoring can occur, for example, when using measurement instruments with detection limits (e.g., viral load tests). In the present study, left-censoring is occurring, due to a number of participants with a score of zero for the SIS-R assessment.

Analogous analyses were conducted to assess associations between polygenic risk and psychotic outcomes. Age, sex, and cannabis use were considered a priori as potential confounders in all analyses described above examining psychotic outcomes (caseness, schizotypy) as these variables are robustly associated with psychosis, and independently of that association, may also be linked to childhood trauma ${ }^{20,25}$ and possibly familial/ genetic risk for schizophrenia ${ }^{21}$. Other analyses (not examining psychotic outcomes) were co-varied for age and gender.

\section{Examination of gene-environment correlation}

Associations of childhood trauma with polygenic risk scores in the siblings and controls were assessed using censored regression models (as left-censoring is occurring due to a number of participants with a minimum value of 1 for childhood trauma), with childhood trauma score as dependent variable, and polygenic risk score as independent variable, again accounting for clustering within families, using robust standard errors. This analysis was performed separately for each group.

In order to assess whether associations of trauma with schizotypy were dependent on genetic risk, censored regression analyses were performed including polygenic risk scores as covariate, childhood trauma as dependent variable, and schizotypy as dependent, while accounting for clustering within families using robust standard errors. Additionally, logistic regressions in a case-control and case-sibling design were performed, including polygenic risk as a covariate, while accounting for clustering within families using robust 
standard errors, to assess influence of genetic risk on associations between childhood trauma and schizophrenia (with childhood trauma as independent and caseness as dependent variables).

\section{Examination of gene-environment interaction}

The interaction effect of childhood trauma and polygenic risk was assessed by using logistic regression in a case-control and case-sibling design. Childhood trauma, polygenic risk, and an interaction term of these variables were added to the model. A recent paper argued that simply adding covariates to statistical models examining gene-environment interaction fails to control for the confounding effect that these variables might have on the gene-environment interaction itsel ${ }^{26}$. The solution to this problem is to add the covariate $x$ environment interaction and the covariate $x$ gene interaction term to the model as well, in order to properly control for the effects of potential confounders. Age, sex, and cannabis use were again considered a priori as potential confounders, as described above, and therefore included as main effects and with the respective covariate $x$ environment interaction and the covariate $\mathrm{x}$ gene interaction terms.

Interaction effects of childhood trauma with polygenic risk scores were similarly assessed in the siblings and controls using the censored regression models, with subclinical psychosis (total SIS-R scores) as outcome.

\section{Power analyses}

Power analyses were performed using the $\mathrm{G}^{*}$ Power software tool version $3.1^{27}$ in order to assess the power to detect small $\left(f^{2}=0.02\right)$, medium $\left(f^{2}=0.15\right)$, and large $\left(f^{2}=0.35\right)$ Cohen's effect $\operatorname{sizes}^{28}$ for the gene-environment correlation analyses, as well as the effect size reported in a recent study reporting a significant, but very small association between cannabis use and polygenic risk ${ }^{29}$. No studies to date have reported on associations between polygenic risk and childhood trauma, yet we have incorporated this effect size in the present study as this may be a good indicator of an expected effect size of polygenic risk associated with environmental exposure. We used the effect size reported for quantity of use, since that measure is most similar to the continuous trauma measure used in our study $\left(0.85 \%\right.$ or an $\mathrm{f}^{2}$ of 0.00857$)$. Analyses showed that the available sample sizes provided adequate power to detect moderate and even small effect sizes, but relatively low power to detect an effect size as reported in the study of Power and colleagues ${ }^{29}$ (Table 2). 
Table 2 Results of the power analyses for the gene-environment correlation analyses

\begin{tabular}{|c|c|c|c|c|}
\hline & \multicolumn{4}{|c|}{ Gene-environment correlation } \\
\hline & $f^{2} 0.00857^{A}$ & small $\left(f^{2}=0.02\right)^{B}$ & medium $\left(f^{2}=0.15\right)$ & large $\left(f^{2}=0.35\right)$ \\
\hline & Power & Power & Power & Power \\
\hline Siblings & 0.52 & 0.87 & 1.00 & 1.00 \\
\hline Controls & 0.38 & 0.72 & 1.00 & 1.00 \\
\hline
\end{tabular}

${ }^{A}$ Effect size $R^{2} 0.85 \%\left(R^{2} 0.0085\right)$ based on previous work ${ }^{29} ; f^{2}=R^{2} / 1-R^{2}$

${ }^{B}$ Cohen's effect size $f^{2}$ (small $=0.02$, medium $=0.15$, large $=0.35$ ) equals $V_{s}$ (proportion of variance explained by a set of predictors) divided by $\mathrm{V}_{\mathrm{E}}$ (residual or error variance) $)^{28}$

For the GxE analyses, we conducted power analyses by simulation using Stata $13^{30}$. Thus, predicted values of the dependent variable (y-hat) were generated, using the formula for the regression, including $\times 1$ (polygenic risk), $\times 2$ (childhood trauma), and the interaction term $(x 1 * x 2): y-h a t={ }^{\prime} b 0^{\prime}+{ }^{\prime} b 1^{\prime *} x 1+{ }^{\prime} b 2{ }^{\prime *} x 2+{ }^{\prime} b 3^{\prime *} \times 1{ }^{*} \times 2$

Random noise was generated around $y$-hat to simulate the error term in the regression equation (residuals). This simulated $y$-variable was analysed using the appropriate regression analysis (including $\mathrm{x} 1$ and $\mathrm{x} 2$ as well as the two interaction terms). This step was repeated 1,000 times. The power is the percentage in which the interaction term is statistically significant. We used the actual main effect of trauma as the reference for the effects size for the interaction. Analyses showed sufficient power at around standardized coefficients (beta) of 0.3 or higher, which is considerably smaller than the main effect of trauma in both the case-sibling (beta 0.6), as well as the case-control comparison (beta 0.9), indicating sufficient power (Table 3). The GxE analysis examining schizotypy revealed a weaker, but significant main effect of trauma in the siblings (beta 0.1) as well as in the controls (beta 0.08). Analyses showed that the power to detect an interaction effect was modest at beta $0.1(0.54)$, and sufficient at beta $0.2(0.99)$ and beta $0.3(1.00)$ in both the siblings and the controls.

Table 3 Results of the power analyses for the gene-environment interaction analyses

\begin{tabular}{llll}
\hline & beta $^{\mathrm{A}}=\mathbf{0 . 2}$ & beta $=\mathbf{0 . 3}$ & beta $=\mathbf{0 . 5}$ and higher \\
\hline Case-control & 0.45 & 0.75 & 1.00 \\
Case-sibling & 0.58 & 0.89 & 1.00 \\
\hline
\end{tabular}

A Beta trauma in case-control design 0.9, beta trauma in case-sibling design 0.6 


\section{RESULTS}

\section{Childhood trauma and psychosis}

Childhood trauma predicted caseness in the case-control and case-sibling comparison (Table 4). The association between childhood trauma and schizotypy was strong and significant in both the siblings (B $0.22,95 \% \mathrm{Cl} 0.16-0.28, \mathrm{p}<0.001$ ) and the controls (B 0.18, $95 \% \mathrm{Cl} 0.09-0.28, \mathrm{p}<0.001)$.

\section{Polygenic risk and psychosis}

The average polygenic risk scores for the patients, their unaffected siblings, and the healthy controls can be found in Table 1. Polygenic risk was not associated with schizotypy in the siblings (B $0.0495 \% \mathrm{Cl}-0.02-0.11, \mathrm{p}=0.184)$ or in the controls $(\mathrm{B}-0.0004,95 \% \mathrm{Cl}$ $-0.03-0.03, p=0.981$ ). The polygenic risk score was however associated with psychotic disorder in both the case-control and the case-sibling design (Table 4).

Table 4 GROUP baseline measurement. Group comparisons (case-control and case-sibling) of associations of childhood trauma and polygenic risk

\begin{tabular}{|c|c|c|c|c|}
\hline & \multicolumn{2}{|c|}{ Case-control } & \multicolumn{2}{|c|}{ Case-sibling } \\
\hline & \multicolumn{2}{|c|}{ OR $(95 \% \mathrm{Cl})$} & \multicolumn{2}{|c|}{ OR $(95 \% \mathrm{Cl})$} \\
\hline & & Adjusted for & & Adjusted for \\
\hline & & polygenic risk & & polygenic risk \\
\hline Childhood trauma & $5.15^{* * *}(3.30-8.03)$ & $6.02 * * *(3.47-10.42)$ & $3.06^{* * *}(2.24-4.19)$ & $3.03 * * *(2.04-4.49)$ \\
\hline Polygenic risk score & $1.90 * * *(1.53-2.36)$ & n.a. & $2.82 * * *(2.22-3.58)$ & n.a. \\
\hline
\end{tabular}

Note: OR, Odds Ratio; Cl, Confidence Interval; *** $\mathrm{p}<0.001$; Odds Ratios adjusted for age and gender

\section{Examination of gene-environment correlation}

Polygenic risk scores were not associated with childhood trauma in the unaffected siblings (B -0.04, 95\% Cl -0.14-0.06, $\mathrm{p}=0.434$ ) and healthy controls (B $0.01,95 \% \mathrm{Cl}-0.04-0.05$, $\mathrm{p}=0.756$ ). Associations between childhood trauma and psychosis remained similar after adding polygenic risk scores as a covariate, on a clinical level (Table 4), as well as subclinical level in the siblings $(\mathrm{B} 0.22,95 \% \mathrm{Cl} 0.14-0.31, \mathrm{p}<0.001)$ and the controls $(\mathrm{B} 0.15,95 \% \mathrm{Cl}$ $0.05-0.25, p=0.004)$.

\section{Examination of gene-environment interaction}

No evidence was found for gene-environment interaction in the case-control (OR 0.88, 95\% $\mathrm{Cl} 0.39-1.97, \mathrm{p}=0.750$ ) or case-sibling design (OR 1.24, 95\% Cl 0.64-2.40, $\mathrm{p}=0.520$ ). Similarly, no gene-environment interaction was found for subclinical psychosis in the controls (B-0.05, $95 \% \mathrm{Cl}-0.15-0.06), \mathrm{p}=0.358$ ) or in the siblings ( $\mathrm{B} 0.11,95 \% \mathrm{Cl}-0.08-0.29, \mathrm{p}=0.265$ ). 


\section{DISCUSSION}

Childhood trauma showed strong and significant associations with psychotic outcomes, both at the clinical (caseness) and at the subclinical level (schizotypy in siblings and controls). The most salient finding of this study was a lack of evidence that this association may be explained by gene-environment correlation. Firstly, there was no association between polygenic risk for schizophrenia and exposure to childhood traumatic experiences. Secondly, the association between childhood trauma and psychotic outcomes was not attenuated by including polygenic risk as a possible confounder. In addition to the lack of gene-environment correlation, we also did not find evidence for gene-environment interaction in the association of childhood trauma with psychosis.

These findings provide further support that childhood trauma contributes causally to the development of psychosis, throughout the entire psychosis continuum, as they argue against the possibility that the association is entirely attributable to gene-environment correlation. However, the power to detect the small association between polygenic risk and environmental exposure, reported elsewhere ${ }^{29}$, was limited, although this reported association was between polygenic risk and cannabis use, not childhood trauma, therefore could only be used as an approximate in the present study. Furthermore, data from a more resent GWAS was used in the present study, which explained considerably more of the variance in schizophrenia ${ }^{12}$. Evidence arguing against gene-environment correlation explaining the association of trauma with psychosis was also provided by Alemany and colleagues ${ }^{31}$. Their study, using a monozygotic twin sample, showed that differences in exposure to childhood trauma were associated with psychotic phenotypic differences within the twin pairs. As these twin pairs were genetically identical, these findings indicate that this phenotypic variability must have been due to differences in environmental exposures to childhood traumatic experiences ${ }^{31}$.

The present study could not rule out the possibility of another alternative non-causal explanation, namely reverse causation ${ }^{32}$, as reverse causation has been shown to be present in previous work by Kelleher and colleagues $^{3}$. Nevertheless, Kelleher and colleagues also provided evidence that reverse causation cannot entirely explain the association between childhood trauma and psychosis, by showing a decrease in psychosis scores after cessation of abusive events ${ }^{3}$, which suggests a causal influence. Furthermore, prospective studies have shown that individuals at Ultra High Risk for psychosis ${ }^{4}$ and healthy (non-psychotic) individuals ${ }^{5}$ exposed to childhood trauma were more likely to transition to clinical psychosis. Additionally, over-reporting of childhood traumatic events by patients with a psychotic disorder has been shown to be unlikely ${ }^{33}$.

Another important finding of the present study is the absence of evidence for geneenvironment interaction. This is consistent with studies using familial risk for psychosis as a proxy for genetic risk, which consistently reveal an absence of significant interactions with childhood adverse events ${ }^{34-36}$. The nature ${ }^{37}$, timing ${ }^{38}$, and duration of traumatic events $^{34}$, as well as possible synergistic effects with other risk factors such as cannabis use $^{39}$, may therefore be more important in determining which exposed individuals will 
develop psychotic experiences. It further seems plausible that 'schizophrenia' patients represent a spectrum of etiological trajectories, each of which may be mediated by distinct biological pathways, interacting with different environmental factors that may vary in terms of their nature, severity and timing ${ }^{40}$, rather than by cumulative scores of genetic risk and/or trauma exposure.

These finding should be interpreted in terms of their strengths and limitations. This study is one of the first studies to use polygenic risk scores (based on the most recent PGC2 data) in order to assess gene-environment interplay associated with psychotic outcomes, using a sample of not only patients with a psychotic disorder, but also their unaffected siblings and unrelated controls. Trained interviewers assessed diagnoses and subclinical psychosis using extensive interviews, and diagnoses were confirmed by psychiatrists. Limitations are that childhood trauma was assessed by retrospective report, although this has been found to yield reliable results, also in psychiatric patients ${ }^{33}$. Furthermore, the variance explained by the current polygenic risk scores is still a matter of debate, but conservative estimations show that the present polygenic risk score is able to explain around $18 \%$ of the variance. This further leaves open the possibility of an association between childhood trauma and future polygenic risk scores explaining a larger proportion of the heritability. Nevertheless, given the power estimations in the present study, showing essentially $100 \%$ power to detect moderate to large effects, it seems unlikely that if such an association between polygenic risk and schizophrenia would exist, this would explain a large proportion of the association between childhood trauma and psychosis. 


\section{REFERENCES}

1. Varese F, Smeets F, Drukker M, et al. Childhood adversities increase the risk of psychosis: a meta-analysis of patient-control, prospective- and cross-sectional cohort studies. Schizophr Bull 2012;38(4):661-671.

2. van Os J, Rutten BPF, Poulton R. Gene-environment interaction in schizophrenia: review of epidemiological findings and future directions. Schiophr Bull 2008;34(6):1066-1082.

3. Kelleher I, Keeley H, Corcoran $\mathrm{P}$, et al. Childhood trauma and psychosis in a prospective cohort study: cause, effect, and directionality. Am J Psychiat 2013;170(7):734-741.

4. Thompson AD, Nelson B, Yuen HP, Lin A, Amminger GP, McGorry PD, Wood SJ, Yung AR. Sexual trauma increases the risk of developing psychosis in an ultra high-risk "prodromal" population. Schizophr Bull May 2014;40(3):697-706.

5. van Nierop M, Janssens M, GROUP. Evidence that transition from health to psychotic disorder can be traced to semi-ubiquitous environmental effects operating against background genetic risk. Plos One 2013;8(11):e76690.

6. Heins $M$, Simons $C$, Lataster $T$, et al. Childhood trauma and psychosis: A case-control and case-sibling comparison across different levels of genetic liability, psychopathology, and type of trauma. Am J Psychiat 2011;168(12):1286-1294.

7. Purcell SM, Wray NR, Stone JL, Visscher PM, O'Donovan MC, Sullivan PF, Sklar P. Common polygenic variation contributes to risk of schizophrenia and bipolar disorder. Nature 2009;460(7256):748-752.
8. Dudbridge F. Power and predictive accuracy of polygenic risk scores. PLoS genetics 2013;9(3):e1003348.

9. Wray NR, Goddard ME, Visscher PM. Prediction of individual genetic risk to disease from genome-wide association studies. Genome res Oct 2007;17(10):1520-1528.

10. Bernstein DP, Stein JA, Newcomb MD, et al. Development and validation of a brief screening version of the Childhood Trauma Questionnaire. Child Abuse \& Neglect 2003;27:169-190.

11. Vollema MG, Ormel J. The reliability of the structured interview for schizotypy-revised. Schizophr Bull 2000;26(3):619-629.

12. Schizophrenia Working Group of the Psychiatric Genomic Consortium. Biological insights from 108 schizophrenia-associated genetic loci. Nature 2014;in press.

13. American Psychiatric Association. Diagnostic and statistical manual of mental disorders. 4th ed., text rev. ed: Washington, DC; 2000.

14. Andreasen NC, Flaum M, Arndt S. The Comprehensive Assessment of Symptoms and History (CASH). An instrument for assessing diagnosis and psychopathology. Arch Gen Psychiat 1992;49(8):615-623.

15. Wing JK, Babor T, Brugha $T$, et al. SCAN. Schedules for Clinical Assessment in Neuropsychiatry. Arch Gen Psychiat 1990;47(6):589-593.

16. NIMH.Genetics.Initiative. Family Interview for Genetic Studies (FIGS). Rockville, Md: National Institute of Mental Health; 1992. 
17. Wechsler D. WAIS-III: Wechsler Adult Intelligence Scale (3rd edition).

Administration and Scoring Manual.

San Antonio, TX: Psychological

Corporation; 1997.

18. Korver N, Quee PJ, Boos HB, Simons

$C$, de Haan L. Genetic Risk and

Outcome of Psychosis (GROUP), a

multi site longitudinal cohort study

focused on gene-environment

interaction: objectives, sample

characteristics, recruitment and

assessment methods. Int J Meth

Psychiat Res 2012;21(3):205-221.

19. Purcell $S$, Neale B, Todd-Brown K, et al. PLINK: a tool set for whole-genome association and population-based linkage analyses. Am J Hum Gen Sep 2007;81(3):559-575.

20. Harley M, Kelleher I, Clarke M, Lynch

F, Arseneault L, Connor D, Fitzpatrick

C, Cannon M. Cannabis use and childhood trauma interact additively to increase the risk of psychotic symptoms in adolescence. Psychol Med 2010;40:1627-1634.

21. van Winkel $R$, Kuepper $R$.

Epidemiological, neurobiological, and genetic clues to the mechanisms linking cannabis use to risk for nonaffective psychosis. Ann Rev Clin Psychol 2014;10:767-791.

22. Andrew G, Peters L. The psychometric properties of the Composite International Diagnostic Interview. Soc Psych Psych Epidem 1998;33:80-88.

23. van Winkel $R$, Genetic Risk and Outcome of Psychosis (GROUP) investigators. Family-based analysis of genetic variation underlying psychosis-inducing effects of cannabis. Arch Gen Psychiat 2011;68(2):148-157.
24. Breen R. Regression models:

Censored, sample selected or truncated data. Thousand Oaks, CA: Sage; 1996.

25. Fisher H, Morgan C, Dazzan P, et al. Gender differences in the association between childhood abuse and psychosis. B J Psychiat 2009;194(4):319-325.

26. Keller MC. Gene x Environment Interaction studies have not properly controlled for potential confounders: the problem and the (simple) solution. Bio Psychiatry 2014;75:18-24.

27. Faul F, Erdfelder E, Buchner A, Lang AG. Statistical power analyses using G*Power 3.1: Tests for correlation and regression analyses. Beh Res Meth 2009;41:1149-1160.

28. Cohen J. Statistical power analysis for the behavioral sciences. Hillsdale, New Jersey: Lawrence Erlbaum Associates; 1988.

29. Power RA, Verweij KJ, Zuhair M, et al. Genetic predisposition to schizophrenia associated with increased use of cannabis. Mol Psychiatry Jun 242014.

30. Statacorp. Stata Statistical Software: Release 13. College Station, TX: StataCorp LP. 2013.

31. Alemany S, Goldberg X, van Winkel R, Gasto C, Peralta V, Fananas L. Childhood adversity and psychosis: examining whether the association is due to genetic confounding using a monozygotic twin differences approach. Eur psychiat : J Ass Eur Psychiatrists May 2013;28(4):207-212. 
32. Van Winkel R, Van Nierop M, MyinGermeys I, van Os J. Childhood Trauma as a Cause of Psychosis: Linking Genes, Psychology, and Biology. Can J Psychiat 2013;58(1):44-51.

33. Fisher HL, Craig TK, Fearon $\mathrm{P}$, et al. Reliability and comparability of psychosis patients' retrospective reports of childhood abuse. Schizophr Bull 2011;37(3):546-553.

34. Arseneault $L$, Cannon $M$, Fisher $H$, Polanczyk G, Moffitt TE, Caspi A. Childhood trauma and children's emerging psychotic symptoms: a genetically sensitive longitudinal cohort study. Am J Psychiat 2011;168:65-72.

35. Fisher H, McGuffin P, Boydell J, et al. Interplay between childhood physical abuse and familial risk in the onset of psychotic disorders. Schizophr Bull 2014;doi: 10.1093/schbul/sbt201.

36. Wigman JTW, Van Winkel R, Ormel J, Verhulst FC, Van Os J, Vollebergh WAM. Early trauma and familial risk in the development of the extended psychosis phenotype in adolescence. Acta Psychiat Scand 2012;126(4):266-273.

37. van Nierop $M$, Lataster $T$, Smeets $F$, et al. Psychopathological mechanisms linking childhood traumatic experiences to risk of psychotic symptoms. Analysis of a large, representative population-based sample. Schizophr Bull 2014;40(suppl.2):S123-S130.
38. Cutajar MC, Mullen PE, Ogloff JR, Thomas SD, Wells DL, Spataro J. Psychopathology in a large cohort of sexually abused children followed up to 43 years. Child Abuse Neg/ Nov 2010;34(11):813-822.

39. Konings $M$, Stefanis N, Kuepper R, de Graaf R, ten Have M, van Os J, Bakoula C, Henquet C. Replication in two independent population-based samples that childhood maltreatment and cannabis use synergistically impact on psychosis risk. Psychol Med Jan 2012;42(1):149-159.

40. EU-GEI. Identifying Gene-

Environment Interactions in Schizophrenia: Contemporary Challenges for Integrated, Large-scale Investigations. Schizophr Bull May 24 2014. 
126 | Surviving Childhood 


\title{
CHAPTER 8
}

\section{Childhood Abuse and Neglect in Relation to the Presence and Persistence of Psychotic and Depressive Symptomatology}

\author{
Martine van Nierop ${ }^{A *}$ \\ Daniëlla S. van Dam ${ }^{B *}$ \\ Wolfgang Viechtbauer ${ }^{A}$ \\ Eva Velthorst ${ }^{\mathrm{B}}$ \\ Ruud van Winkel ${ }^{\mathrm{AC}}$
}

Genetic Risk and Outcome of Psychosis (GROUP) investigators ${ }^{\S}$

\author{
* These authors contributed equally \\ $\S$ The GROUP Investigators are: \\ Richard Bruggeman ${ }^{\mathrm{D}}$ \\ Wiepke Cahn ${ }^{\mathrm{E}}$ \\ Lieuwe de Haan $^{\mathrm{B}}$ \\ René S. Kahn ${ }^{\mathrm{E}}$ \\ Carin J. Meijer ${ }^{\mathrm{B}}$ \\ Inez Myin-Germeys ${ }^{\mathrm{A}}$ \\ Jim van Os ${ }^{\mathrm{AF}}$ \\ Durk Wiersma ${ }^{\mathrm{D}}$
}

\footnotetext{
A Maastricht University Medical Centre, South Limburg Mental Health Research and Teaching Network, EURON, Maastricht, The Netherlands

B Academic Medical Centre University of Amsterdam, Department of Psychiatry, Amsterdam The Netherlands

C University Psychiatric Center Katholieke Universiteit Leuven, campus Kortenberg, Leuvensesteenweg, Kortenberg, Belgium D University Medical Center Groningen, Department of Psychiatry, University of Groningen, The Netherlands

E University Medical Center Utrecht, Department of Psychiatry, Rudolf Magnus Institute of Neuroscience, The Netherlands

F King's College London, King's Health Partners, Department of Psychosis Studies, Institute of Psychiatry,

London, United Kingdom
}

Psychological Medicine 2014; doi:10.1017/S0033291714001561 


\section{ABSTRACT}

\section{Background}

The association between childhood trauma and psychotic and depressive symptomatology is well established. However, less is known about the specificity and course of these symptoms in relation to childhood trauma.

\section{Methods}

In a large sample $(\mathrm{N}=2765)$ of patients with psychosis $(\mathrm{N}=1119)$, their siblings $(\mathrm{N}=1057)$, and controls $(\mathrm{N}=589)$, multivariate (mixed-effects) regression analyses with multiple outcomes were performed to examine the association between childhood trauma and psychotic and depressive symptomatology over a three year period.

\section{Results}

A dose-response relationship was found between childhood trauma and psychosis. Abuse was more strongly associated with positive symptoms than with negative symptoms whereas the strength of the associations between neglect and positive and negative symptoms was comparable. In patients, similar associations between childhood trauma and psychotic or depressive symptoms were found, and in siblings and controls, stronger associations were found between trauma and depressive symptomatology. Childhood trauma was not related to a differential course of symptoms over a 3-year time period.

\section{Conclusions}

In congruence with earlier work, our findings suggest that childhood trauma, and abuse in particular, is associated with (subthreshold) psychosis. However, childhood trauma does not seem to be associated with a differential course of symptoms, nor does it uniquely heighten the chance of developing (subthreshold) psychotic symptomatology. Our results indicate that trauma may rather contribute to a shared vulnerability for psychotic and depressive symptoms. 


\section{INTRODUCTION}

The association between childhood abuse and neglect (hereafter childhood trauma) and psychosis is well established. A growing number of prospective cohort studies, casecontrol, and cross-sectional studies found strong associations between childhood trauma and the development of psychotic disorders ${ }^{1}$. However, in studies examining this association, there are still important issues that have received limited attention.

One of these issues concerns the specificity of type of childhood trauma in relation to different symptom domains of psychosis. That is, various types of early trauma may have different effects on neurodevelopmental, social, and emotional development ${ }^{2}$ and are therefore possibly associated with different symptoms ${ }^{3,4}$. A study conducted by Heins and colleagues addressed this issue by comparing effect sizes of abuse and neglect in relation to distinct psychosis symptom domains in patients, siblings, and controls ${ }^{5}$. They found the strongest associations between abuse and (subthreshold) positive symptoms, whereas neglect was more strongly related to general psychopathology.

Although the study of Heins and colleagues ${ }^{5}$ has increased our understanding of specific associations between childhood trauma and psychosis, to date there are more sophisticated statistical techniques to assess the influence of childhood trauma on different, yet often co-occurring symptom clusters (e.g. positive, negative, and general symptoms in psychotic disorders). Specifically, multivariate (mixed-effects) regression allows examination of the unique influence of childhood trauma in relation to multiple symptom domains, and direct comparison of which association is stronger ${ }^{6}$.

It is also unclear to what extent childhood trauma specifically increases the chance of developing psychotic symptoms in comparison to other psychiatric symptoms. Childhood trauma is not only related to a greater risk of developing psychotic disorders, but has also been linked to a variety of other psychiatric disorders later in life, such as depression, affective psychosis, and different anxiety disorders including posttraumatic stress disorder ${ }^{7}$. An important question in this regard is whether childhood trauma uniquely heightens the chance of developing (subtreshold) psychotic symptomatology in persons who are genetically vulnerable for developing psychotic symptoms.

Another issue that needs evaluation is the association between childhood trauma and course of symptoms over time in patients with psychosis. General population studies suggest that childhood trauma not only predicts the development of subclinical psychotic symptoms but is also related to higher symptom levels over time ${ }^{8-14}$. However, the literature about the association between childhood trauma and course of symptoms in patients with psychosis is sparse and results are contradictory ${ }^{15,16}$. Although it is known that childhood trauma is related to heightened symptoms levels $s^{5,17,18}$, it is not clear how these symptoms evolve over time.

The literature on the impact of childhood trauma on the course of symptoms in affective 
disorders is more consistent. Although only a few studies have investigated the association between childhood trauma and symptomatology over time, it has been reported that the presence of childhood trauma leads to higher levels of manic, depressive, and anxiety symptoms over time in patients with bipolar ${ }^{19,20}$ and depressive disorder ${ }^{21,22}$.

Investigating childhood trauma in relation to the course of symptoms over time is required to estimate the long-term impact of trauma on symptomology. It is also of interest to examine whether childhood trauma is related to a differential course of symptoms (that is, whether symptoms in the trauma group increase or decrease to a similar extent as compared to the non-trauma group), which to the best our knowledge, no study has yet examined.

The aims of the present study were (i) to replicate the study of Heins and colleagues ${ }^{5}$ in an independent and larger sample of patients with psychosis, siblings, and control subjects, by using more advanced statistical methods accounting for the unique influence of childhood trauma in relation to multiple symptom domains, (ii) to examine whether childhood trauma is more strongly related to (subtreshold) psychotic than affective symptoms in subjects at genetic risk of psychosis, and (iii) to examine whether childhood trauma is related to a differential course of symptoms.

\section{METHODS}

\section{Subjects}

This research is part of a longitudinal observational study called the 'Genetic Risk and Outcome of Psychosis Project (GROUP) ${ }^{23}$. The GROUP study investigates the vulnerability and resilience factors for the development of a psychotic disorder and the variation in the course of the illness. The full sample consists of patients $(n=1119)$, their siblings ( $n=1057)$, and a control group $(n=589)$.

In representative geographical areas in the Netherlands and Belgium, patients were identified through clinicians working in regional psychotic disorder services, whose caseload was screened for inclusion criteria. Subsequently, a group of patients presenting at these services either as out-patients or in-patients were recruited for the study. Patients were recruited from four university departments of psychiatry in the Netherlands (Amsterdam, Groningen, Maastricht, and Utrecht) and affiliated mental health care institutions. Heins and colleagues ${ }^{5}$ used the sample that was recruited in Maastricht (patients: $n=306$; siblings: $n=289$; controls: $n=244$ ). Thus, for the replication analyses, only subjects recruited from the other sites were used: Amsterdam (patients: $n=283$; siblings: $n=258$; controls: $n=104)$, Groningen (patients: $n=287$; siblings: $n=273$; controls: 84 ), and Utrecht (patients: $n=243$; siblings: $n=237$; controls: $n=157$ ). For all other analyses, the full sample (including the Maastricht sample) was used. 


\section{Inclusion criteria}

Patients were eligible to be included in the study if: (1) they were aged between 16 and 50 years, (2) they met the Diagnostic and Statistical Manual of Mental Disorders, Fourth Edition (DSM-IV) criteria for a non-affective psychotic disorder (schizophrenia, schizophreniform disorder, schizoaffective disorder, delusional disorder, psychotic disorder NOS), (3) their first contact with mental health care had occurred less than ten years ago, (4) they were able to communicate the Dutch language, and (5) had one or more siblings volunteering to participate in the research. Except for the DSM-IV and the mental health care criteria, similar criteria were applied to the siblings and the controls.

Control subjects were selected through random mailings to addresses in the catchment areas of the cases. The majority of mental health care services in the Netherlands and a substantial number of mental health services in Dutch-speaking Belgium took part in the GROUP study. Representativeness of the control sample was maximized as the control sample (i) was collected from the same geographical area as the case in the relevant mental health service, (ii) was sufficiently large to allow for chance variation, and (iii) was frequency-matched in age and sex distribution to the siblings.

\section{Exclusion criteria}

Subjects were excluded if their estimated level of intelligence was below 70, as assessed with the short form of the Wechsler Adult Intelligence Scale [WAIS] - III ${ }^{24}$. When siblings fulfilled criteria for a psychotic disorder, they were included in the patient group. Controls were excluded if they had a history of psychosis or if they had a first-degree family member diagnosed with a psychotic disorder. To confirm absence of a family history of psychotic disorders in the controls, the Family Interview for Genetic studies (FIGS ${ }^{25}$ ) was conducted, with the control as informant, to establish absence of first degree relatives with a psychotic disorder.

\section{Measures}

The Comprehensive Assessment of Symptoms and History $\left(\mathrm{CASH}^{26}\right)$ or the Schedules for Clinical Assessment in Neuropsychiatry (SCAN 2.127) (Groningen site) was used to assess DSM-IV diagnosis at baseline and at the 3-year follow-up. The CASH includes the Scale for the Assessment of Positive Symptoms (SAPS, with 34 items measured on a Likert scale ranging from 0 [absent] to 5 [severe] ${ }^{28}$ ) and the 21-item Scale for the Assessment of Negative Symptoms $\left(\mathrm{SANS}^{29}\right.$ ). The SCAN is a semi-structured computer-based interview to assess psychiatric symptoms. Level of functioning was assessed by The Global Assessment of Functioning (GAF) scale ${ }^{30}$. This scale defined a symptom score (GAF-S) and a functioning score (GAF-F), reflecting the severity of symptoms and the level of daily functioning. 


\section{Childhood Trauma}

Childhood trauma was measured (at baseline at the Maastricht site, the other sites added this measure to the protocol at the follow-up measurement) with the Dutch version of the Childhood Trauma Questionnaire Short Form (CTQ-SF ${ }^{31,32}$ ), a 25-item self-report questionnaire rated on a 5-point Likert scale with good internal consistency, reliability, and validity ${ }^{32}$.

The CTQ measures: physical abuse (bodily assaults on a child by an adult or older person that posed a risk of or resulted in injury); physical neglect (the failure of caretakers to provide for a child's basic physical needs, including food, shelter, clothing, safety, and health care); sexual abuse (unwanted sexual contact or conduct between a child younger than 18 years of age and an adult or older person); emotional abuse (verbal assaults on a child's sense of worth or well-being or any humiliating or demeaning behaviour directed toward a child by an adult or older person) ; and emotional neglect (the failure of caretakers to meet children's basic emotional and psychological needs, including love, belonging, nurturance, and support), all occurring before the age of 17 .

For all analyses, three different trauma scales were used: total trauma (all 5 trauma types combined), abuse (emotional, physical, and sexual abuse), and neglect (emotional and physical neglect). Each type of trauma was dichotomized in high trauma and low trauma, using the 80th percentile of trauma scores of controls, conform previous work ${ }^{5}$, enabling us to present results based on comparable methods.

\section{Symptomatology}

The positive and negative subscales of the Positive and Negative Syndrome Scale (PANSS ${ }^{33}$ ) were used to assess the severity of a variety of symptoms in the patient population at baseline and at the 3-year follow-up. The PANSS is a 30-item interview and originally consisting of three subscales (the positive scale [e.g. delusions/hallucinations], the negative scale [e.g. blunted affect, difficulty in abstract thinking], and a general psychopathology scale [e.g. depression, feelings of guilt]), and is scored on a 7-point scale ranging from 1 (absent) to 7 (very severe).

Siblings and controls were assessed at baseline and the 3-year follow-up with the Structured Interview for Schizotypy-Revised (SIS-R ${ }^{34-36}$ ) to measure schizotypy. Guided by previous research ${ }^{37}$, item scores were reduced a priori to two dimensional scores, representing the means of positive schizotypy items (e.g. referential thinking, psychotic phenomena, and derealisation; range 0-2.7), and negative disorganised schizotypy items (e.g. social isolation and introversion; range $0-1.8$ ).

Depressive symptoms in siblings and controls were assessed at baseline and the 3-year follow-up with the Community Assessment of Psychic Experiences (CAPE; www.cape42. homestead.com). The CAPE was developed for assessing self-reports of psychotic and depressive symptoms. For the purpose of this study, only the depression scale was used, as self-reports of psychotic experiences may yield high numbers of false positives ${ }^{38}$. 
Each of the eight items was rated in terms of frequency, on a scale of 0 (never) to 3 (almost always).

Depressive symptoms in patients were assessed at the 3-year follow-up with the Calgary Depression Scale $\left(\mathrm{CDS}^{39}\right)$, an interviewed-based scale developed specifically for assessing depressive symptoms in patients with psychosis. Each of the nine items was rated in terms of severity, on a scale of 0 (absent) to 3 (severe). The CDS rather than the CAPE was used for the assessment of depression in the patients, as this measure was specifically designed for use in patients with a psychotic disorder.

\section{Cannabis use}

Cannabis use was assessed at baseline and 3-year follow-up with the Composite International Diagnostic Interview $\left(\mathrm{CIDI}^{40}\right)$. Consistent with previous research in this sample ${ }^{41}$, the cannabis pattern of use during the lifetime period of heaviest use was considered most informative and used for analysis: none (0), less than weekly (1), weekly (2), and daily (3).

\section{Procedure}

The study protocol was approved centrally by the Ethical Review Board of the University Medical Centre Utrecht and subsequently by local review boards of each participating institute. All subjects gave written informed consent in accordance with the committee's guidelines. Assessments by trained research assistants took place at one of the participating regional psychosis departments or at the academic centres.

\section{Statistical analysis}

To determine the relationship between trauma and psychotic disorder, we used the assessments of trauma and diagnoses. Psychotic symptoms, schizotypy, and depressive symptoms were assessed at baseline and follow-up, and analyses of these symptoms included both measurements, taking into account the within-person level clustering of data. A priori confounders added to all analyses were age, gender, and cannabis use ${ }^{42}$. All analyses were performed using Stata 11 . Analyses were repeated including ethnicity (white, non-white), family history of depression, and family history of psychosis as potential confounders ${ }^{43,44}$. 


\section{Replication analyses}

To replicate earlier findings of an association of trauma and psychotic disorder or psychotic symptoms in the Maastricht sample ${ }^{5}$, analyses were performed using the Amsterdam, Groningen, and Utrecht sample.

\section{Between-group comparisons}

For the assessment of the association of total trauma, abuse, or neglect with psychotic disorder, we used a case-control, case-sibling, and sibling-control design. We conducted logistic regression analyses and accounted for dependent observations (clustering of families). In addition, a dose-response relationship between trauma and psychotic disorder was investigated in all groups by dividing the total trauma score of the controls in four quartiles and applying logistic regression analyses, accounting for dependence of observations by clustering for families.

\section{Childhood trauma and symptoms}

Multilevel regression analyses (XTMIXED command) were carried out to investigate the association between trauma and psychotic symptoms or schizotypy. Instead of analyzing the impact of trauma on each symptom category separately (which would only indicate whether trauma is significantly related to each symptom category), these (multivariate) multilevel models assess the differential impact of trauma on positive or negative symptoms within the same model. This approach is therefore preferable in the same sense that subgroup analyses in clinical trials should be conducted by testing proper interactions terms instead of analyzing subgroups separately ${ }^{45}$. The model (Box 1 ) included a dummy variable to distinguish between the two symptom categories. Random effects were added to the multilevel model to account for clustering in families, and to account for clustering within subjects. This analysis was performed separately for total trauma, abuse, and neglect.

Box 1 Mathematical formula for multilevel regression analysis with multiple outcomes, used to assess cross-sectional associations of trauma and positive or negative symptoms.

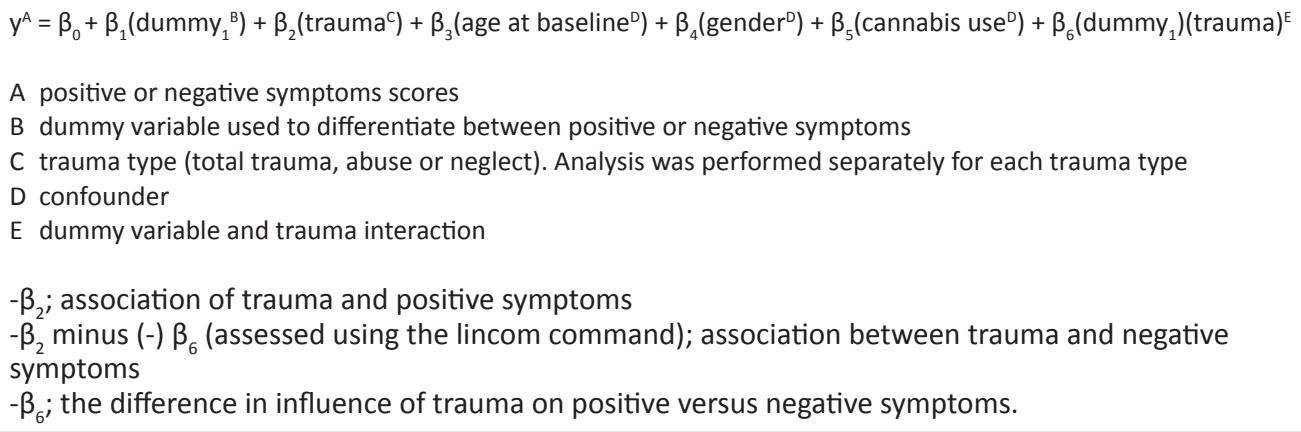




\section{Childhood trauma as a risk factor for developing psychosis or depression}

For the assessment of the differential association of trauma and relevant symptom clusters (positive and negative psychotic symptoms or schizotypy and depression, see Figure 1a), multilevel regression analyses (XTMIXED command) were carried out, using the full sample (all sites). For this model, a second dummy was added to the previous model, allowing the estimation of the differential impact of trauma on the three symptom categories. Where available, both measurements were used in the analyses, while accounting for clustering within subjects and within families. This analysis was performed separately for total trauma, abuse, and neglect.

\section{Type of trauma}

The analyses described above only allow for inferences on differential impact of trauma for symptoms (i.e. the differential impact of trauma on one symptom cluster versus another), not for differences of influence of different types of trauma (i.e. influence of abuse versus neglect on symptom development, see Figure 1b). We assessed differences of influence of abuse or neglect on positive, negative, or depressive symptoms in all three groups, using the XTMIXED command. Both the baseline and first follow-up measurements were used. Random effects were added to the multilevel model to account for clustering in families and to account for clustering within subjects. We performed separate analyses for each group, and each symptom cluster as dependent variable. Both abuse and neglect were added as independent variables. Post-hoc analyses were performed to assess differences in effect sizes of abuse or neglect using the LINCOM command.

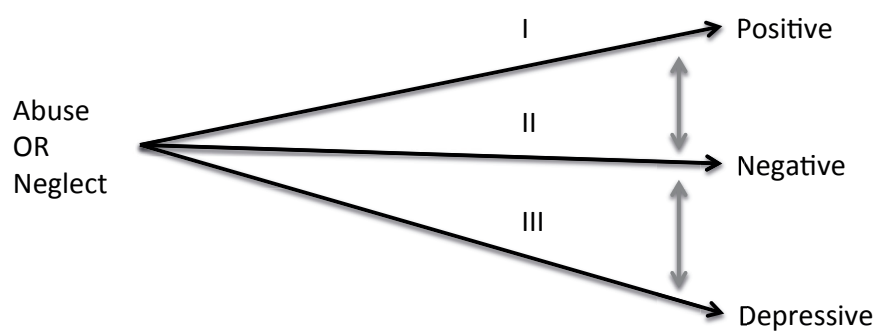

Figure 1a specificity of trauma for symptoms. Testing the differences of the effect sizes of (e.g.) abuse in association with each of the three symptom clusters, by comparing the B coefficients of arrows I, II and III.

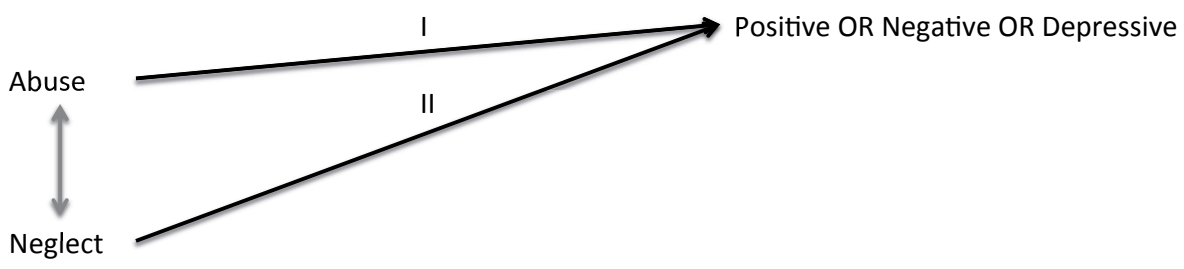

Figure 1b specificity of type of trauma for symptoms. Testing the differences of the effect sizes of (e.g.) abuse and positive symptoms, versus neglect and positive symptoms, by comparing the B coefficients of arrows I and II. 


\section{Influence of childhood trauma on course of symptoms}

In order to assess specificity (differential association between trauma and positive versus negative symptoms or schizotypy) and course of these symptoms, multilevel regression analyses (XTMIXED command) were carried out, using the full sample. All measurements of symptoms at baseline and the follow-up were used in the analysis. For this model, additional two-way and three-way interaction terms were added to the previous model, to estimate (a) the (differential) impact of trauma on the two symptom types at baseline, (b) the (differential) impact of trauma on the two symptom dimensions at follow-up, (c) the (differential) course of symptoms over time, and (d) how the (differential) course of symptoms over time was impacted by trauma (Box 2). This analysis was performed separately for total trauma, abuse, and neglect.

Box 2 Mathematical formula for multilevel regression analysis with multiple outcomes, used to assess both cross-sectional associations of trauma and symptoms, as well as developmental course of symptoms, as influenced by trauma.

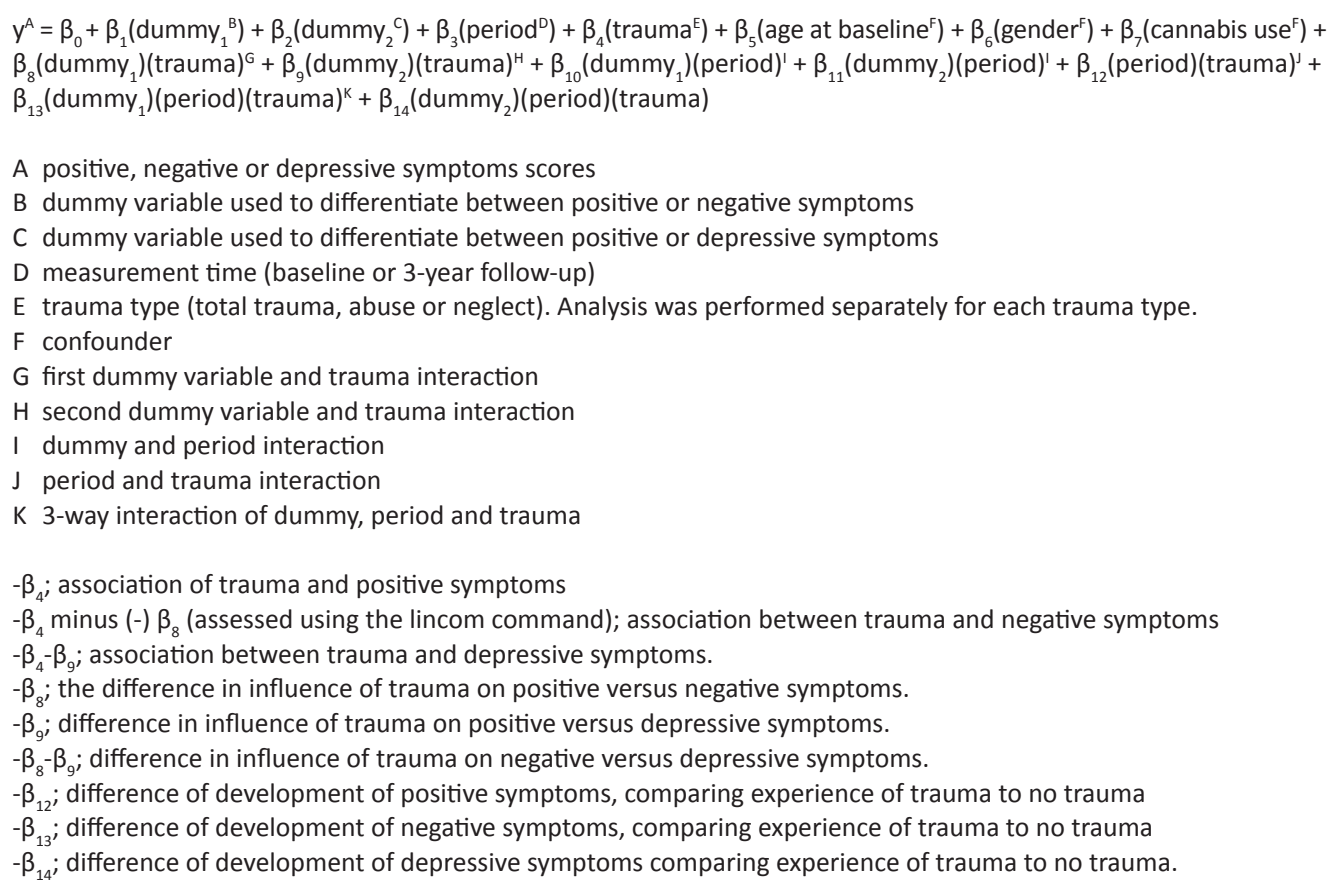




\section{RESULTS}

\section{Subject Characteristics}

This study included 1119 patients, 1057 siblings, and 589 controls at baseline (see Table 1 for characteristics). Of these, $75 \%(n=2074)$ were assessed at follow-up (controls: $78 \%$, $\mathrm{n}=462$; siblings: $77 \%, \mathrm{n}=810$; patients: $72 \%, \mathrm{n}=802$ ); 633 patients completed the CTQ (baseline), the CDS (3-year follow-up), and the PANSS (baseline and 3-year follow-up). Baseline and follow-up data for the CTQ (baseline), the SIS-R and CAPE were available for 645 siblings and 407 controls. The other participants were excluded from the symptom analyses.

Patients who had to be excluded because of incomplete data did not significantly differ in terms of age or sex compared with patients who did participate on all measures. However, significantly more patients from a different ethnic background were excluded from analyses $(t=6.30, p<0.001)$. Controls excluded were significantly younger $(t=-3.03$, $p=0.003)$, were more often male $(t=-1.98, p=0.048)$ and non-white $(t=2.69, p=0.007)$. The siblings who did not participate on all measures did not significantly differ in terms of age or sex, but were more often non-white $(t=5.16, p<0.001)$.

A description of the replication sample (recruited at the Amsterdam, Groningen and Utrecht sites) is shown in Table 1. Of these, 429 patients completed the CTQ (baseline), the CDS (3-year follow-up), and the PANSS (baseline and 3-year follow-up). 497 siblings and 251 controls participated in all measurements. In analyses using the replication sample, only subjects who participated on all measurements were included.

In the replication sample, patients who were excluded from analyses did not significantly differ from participants in terms of age or sex. Patients who did not participate on all measures were, however, more often non-white than patients who did not $(t=5.77, p<0.001)$. Controls who did not participate on all measurements were younger $(t=-2.38, p=0.018)$ and more often non-white than controls available for all measurements $(t=2.19, p=0.029)$. Siblings who did not participate on all measurements did not differ in age or sex; however, they were more often non-white $(t=5.15, p<0.001)$.

\section{Replication analyses}

\section{Between-group comparisons}

In the replication sample, total trauma, abuse, and neglect were all associated with psychotic disorder in the case-control as well as in the case-sibling comparisons (Table 2), with evidence for positive dose-response relationships in both analyses (Table 3). Further, siblings reported more childhood trauma compared with the controls (Table 2). 


\section{Childhood trauma and symptoms}

In all three groups, total trauma, abuse, and neglect were all associated with more severe positive and negative symptoms or schizotypy (table 4). However, abuse was more strongly associated with positive than with negative symptoms or schizotypy, whereas this differential impact was not found for neglect. These results remained robust after adding ethnicity, family history of depression, and family history of psychosis as confounders.

Table 1 Sociodemographic and clinical characteristics of patients, siblings and healthy controls

\begin{tabular}{|c|c|c|c|c|c|c|}
\hline Full sample & \multicolumn{2}{|c|}{ Patients ( $\mathrm{N}=1119$ ) } & \multicolumn{2}{|c|}{ Siblings (N=1057) } & \multicolumn{2}{|c|}{ Controls ( $N=589)$} \\
\hline Male gender N (\%) & $852(76)$ & & $482(46)$ & & $269(46)$ & \\
\hline $\begin{array}{l}\text { Age at baseline (years), mean } \\
\text { (SD) }\end{array}$ & $27.6(8.0)$ & & $27.8(8.3)$ & & $30.4(10.6)$ & \\
\hline Cannabise use ${ }^{\mathrm{A}}$, mean (SD) & $1.21(1.35)$ & & $0.58(1.01)$ & & $0.38(0.82)$ & \\
\hline \multicolumn{7}{|l|}{ GAF disability score, mean (SD) } \\
\hline Baseline & $55.8(16.2)$ & & -- & & -- & \\
\hline 3-year follow-up & $60.1(16.4)$ & & -- & & -- & \\
\hline \multirow[t]{3}{*}{ GAF symptom score, mean (SD) } & $56.7(16.2)$ & & & & & \\
\hline & $59.4(16.3)$ & & & & & \\
\hline & Low trauma & High trauma & $\begin{array}{l}\text { Low } \\
\text { trauma }\end{array}$ & High trauma & Low trauma & $\begin{array}{l}\text { High } \\
\text { trauma }\end{array}$ \\
\hline \multicolumn{7}{|c|}{ Positive symptoms/schizotypy ${ }^{\mathrm{B}}$, mean (SD) } \\
\hline Baseline & $1.65(0.67)$ & $2.01(0.91)$ & $0.37(0.39)$ & $0.57(0.48)$ & $0.31(0.32)$ & $\begin{array}{l}0.53 \\
(0.48)\end{array}$ \\
\hline 3-year follow-up & $1.47(0.59)$ & $1.70(0.69)$ & $0.30(0.29)$ & $0.53(0.38)$ & $0.25(0.27)$ & $\begin{array}{l}0.41 \\
(0.30)\end{array}$ \\
\hline \multicolumn{7}{|c|}{ Negative symptoms/schizotypyc ${ }^{\mathrm{c}}$, mean (SD) } \\
\hline Baseline & $1.81(0.80)$ & $2.01(0.94)$ & $0.25(0.23)$ & $0.34(0.29)$ & $0.24(0.22)$ & $\begin{array}{l}0.31 \\
(0.26)\end{array}$ \\
\hline 3-year follow-up & $1.60(0.64)$ & $1.72(0.79)$ & $0.28(0.23)$ & $0.42(0.29)$ & $0.25(0.21)$ & $\begin{array}{l}0.31 \\
(0.24)\end{array}$ \\
\hline \multicolumn{7}{|l|}{ Depressive symptoms ${ }^{\mathrm{D}}$, mean (SD) } \\
\hline Baseline & --- & --- & $0.58(0.36)$ & $0.81(0.44)$ & $0.53(0.29)$ & $\begin{array}{l}0.84 \\
(0.45)\end{array}$ \\
\hline 3-year follow-up & $1.40(0.60)$ & $1.58(0.74)$ & $0.45(0.36)$ & $0.69(0.46)$ & $0.39(0.30)$ & $\begin{array}{l}0.62 \\
(0.46)\end{array}$ \\
\hline \multicolumn{7}{|c|}{ Trauma scores dichotomized by $80^{\text {th }}$ percentile of healthy controls scores, $\mathrm{N}$ high trauma (\%) } \\
\hline Total trauma score & $336(44)$ & & $202(25)$ & & $94(19)$ & \\
\hline Abuse score & $336(44)$ & & $214(26)$ & & $101(20)$ & \\
\hline Neglect score & $311(41)$ & & $203(25)$ & & 95 (19) & \\
\hline \multicolumn{7}{|c|}{ Continuous trauma scores, mean (SD) } \\
\hline Total trauma score & $1.61(0.50)$ & & $1.41(0.41)$ & & $1.34(0.35)$ & \\
\hline Abuse score & $1.44(0.52)$ & & $1.26(0.40)$ & & $1.22(0.34)$ & \\
\hline Neglect score & $1.86(0.63)$ & & $1.64(0.55)$ & & $1.53(0.49)$ & \\
\hline
\end{tabular}


Table 1 Continued

\begin{tabular}{|c|c|c|c|c|c|c|}
\hline Replication sample ${ }^{\mathrm{E}}$ & \multicolumn{2}{|c|}{ Patients ( $\mathrm{N}=813$ ) } & \multicolumn{2}{|c|}{ Siblings ( $N=768$ ) } & \multicolumn{2}{|c|}{ Controls ( $\mathrm{N}=345)$} \\
\hline Male gender N (\%) & $634(78)$ & & $353(46)$ & & $188(54)$ & \\
\hline $\begin{array}{l}\text { Age at baseline (years), mean } \\
\text { (SD) }\end{array}$ & $27.5(7.8)$ & & $27.9(8.1)$ & & $29.5(9.9)$ & \\
\hline \multirow[t]{2}{*}{ Cannabis use $\mathrm{A}^{\mathrm{A}}$, mean (SD) } & $1.25(1.35)$ & & $0.56(1.0)$ & & $0.46(0.90)$ & \\
\hline & Low trauma & High trauma & $\begin{array}{l}\text { Low } \\
\text { trauma }\end{array}$ & High trauma & Low trauma & $\begin{array}{l}\text { High } \\
\text { trauma }\end{array}$ \\
\hline \multicolumn{7}{|c|}{ Positive symptoms/schizotypy ${ }^{\mathrm{B}}$, mean (SD) } \\
\hline Baseline & $1.66(0.65)$ & $2.05(0.84)$ & $0.23(0.29)$ & $0.40(0.36)$ & $0.17(0.22)$ & $\begin{array}{l}0.30 \\
(0.30)\end{array}$ \\
\hline 3-year follow-up & $1.45(0.53)$ & $1.81(0.70)$ & $0.23(0.27)$ & $0.41(0.35)$ & $0.22(0.26)$ & $\begin{array}{l}0.39 \\
(0.30)\end{array}$ \\
\hline \multicolumn{7}{|c|}{ Negative symptoms/schizotypy ${ }^{\mathrm{C}}$, mean (SD) } \\
\hline Baseline & $1.90(0.73)$ & $2.25(0.88)$ & $0.22(0.24)$ & $0.30(0.27)$ & $0.18(0.19)$ & $\begin{array}{l}0.27 \\
(0.21)\end{array}$ \\
\hline 3-year follow-up & $1.64(0.61)$ & $1.89(0.84)$ & $0.24(0.24)$ & $0.39(0.28)$ & $0.23(0.22)$ & $\begin{array}{l}0.36 \\
(0.29)\end{array}$ \\
\hline \multicolumn{7}{|c|}{ Trauma scores dichotomized by $80^{\text {th }}$ percentile of healthy controls scores, $\mathrm{N}$ high trauma (\%) } \\
\hline Total trauma score & $196(41)$ & & $125(23)$ & & $47(18)$ & \\
\hline Abuse score & $196(41)$ & & $145(26)$ & & 49 (19) & \\
\hline Neglect score & $184(39)$ & & $123(22)$ & & $48(18)$ & \\
\hline \multicolumn{7}{|c|}{ Continuous trauma scores, mean (SD) } \\
\hline Total trauma score & $1.58(0.48)$ & & $1.40(0.39)$ & & $1.33(0.33)$ & \\
\hline Abuse score & $1.41(0.50)$ & & $1.26(0.37)$ & & $1.20(0.31)$ & \\
\hline Neglect score & $1.83(0.61)$ & & $1.62(0.53)$ & & $1.52(0.46)$ & \\
\hline
\end{tabular}

Note: GAF, Global Assessment of Functioning; SD, Standard Deviation

A Cannabis use assessed as frequency of use in the most intensive period lifetime on a scale of 0 (none) to 3 (daily).

${ }^{B}$ Positive symptoms measured with the Positive and Negative Syndrome Scale (PANSS) in patients, positive schizotypy measured with the Structured Interview for Schizotypy - Revised (SIS-R) in siblings and controls.

${ }^{c}$ Negative symptoms measured with the PANSS in patients, negative schizotypy measured with the SIS-R in siblings and controls.

${ }^{D}$ Depressive symptoms measured with the Calgary Depression Scale (CDS) at 3-year follow-up only in patients, depressive symptoms measured with the Community Assessment of Psychic Experiences (CAPE) at baseline and follow-up in siblings and controls.

E Replication sample includes participants from the Amsterdam, Groningen, and Utrecht sites only

Table 2 Association of all types of trauma and psychotic disorder. Case-control, case-sibling and sibling-control comparisons

\begin{tabular}{lllllll}
\hline & Case versus control & & Case versus sibling & \multicolumn{3}{l}{ Sibling versus control } \\
& OR $(95 \% \mathrm{Cl})$ & p-value & OR $(95 \% \mathrm{Cl})$ & p-value & OR $(95 \% \mathrm{Cl})$ & -value \\
\hline Total trauma & $3.11(2.06-4.70)$ & $<0.001$ & $2.57(1.92-3.43)$ & $<0.001$ & $1.28(0.86-1.91)$ & 0.219 \\
Abuse & $3.24(2.16-4.86)$ & $<0.001$ & $2.23(1.65-3.00)$ & $<0.001$ & $1.51(1.03-2.21)$ & 0.035 \\
Neglect & $2.56(1.71-3.83)$ & $<0.001$ & $2.15(1.61-2.87)$ & $<0.001$ & $1.32(0.90-1.95)$ & 0.158 \\
\hline
\end{tabular}

Note: OR, Odds Ratio; $\mathrm{Cl}$, Confidence Interval; A priori corrected for age, gender and cannabis use 


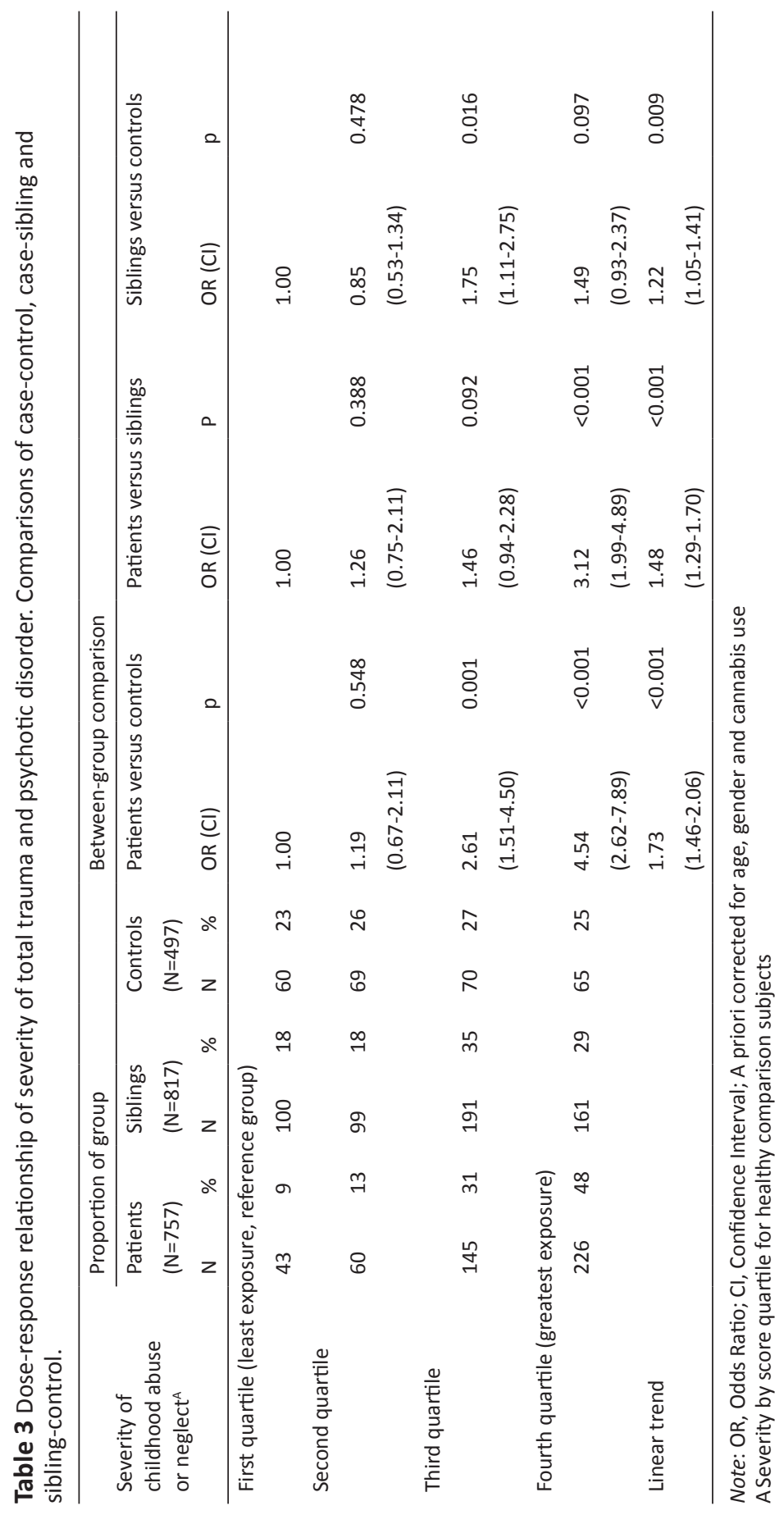


Table 4 Associations of trauma and PANSS symptoms (patients) or SIS-R schizotypy (siblings and controls)

\begin{tabular}{|c|c|c|c|c|}
\hline Type of childhood trauma and group & B coefficient $(95 \% \mathrm{Cl})$ & p-value & Comparison of effect sizes ${ }^{A}$ & p-value \\
\hline \multicolumn{5}{|l|}{ Total trauma } \\
\hline \multicolumn{5}{|l|}{ Patients } \\
\hline Positive symptoms & $0.35(0.24-0.45)$ & $<0.001$ & $-0.07(-0.18-0.04)$ & 0.202 \\
\hline Negative symptoms & $0.27(0.17-0.38)$ & $<0.001$ & & \\
\hline \multicolumn{5}{|l|}{ Siblings } \\
\hline Positive schizotypy & $0.16(0.11-0.21)$ & $<0.001$ & $-0.06(-0.10--0.02)$ & 0.004 \\
\hline Negative schizotypy & $0.10(0.05-0.15)$ & $<0.001$ & & \\
\hline \multicolumn{5}{|l|}{ Controls } \\
\hline Positive schizotypy & $0.16(0.10-0.22)$ & $<0.001$ & $-0.04(-0.11-0.02)$ & 0.181 \\
\hline Negative schizotypy & $0.11(0.05-0.17)$ & $<0.001$ & & \\
\hline \multicolumn{5}{|l|}{ Abuse } \\
\hline \multicolumn{5}{|l|}{ Patients } \\
\hline Positive symptoms & $0.36(0.25-0.47)$ & $<0.001$ & $-0.19(-0.30--0.08)$ & 0.001 \\
\hline Negative symptoms & $0.17(0.06-0.28)$ & 0.002 & & \\
\hline \multicolumn{5}{|l|}{ Siblings } \\
\hline Positive schizotypy & $0.15(0.10-0.19)$ & $<0.001$ & $-0.04(-0.08--0.003)$ & 0.035 \\
\hline Negative schizotypy & $0.11(0.06-0.15)$ & $<0.001$ & & \\
\hline \multicolumn{5}{|l|}{ Controls } \\
\hline Positive schizotypy & $0.18(0.12-0.24)$ & $<0.001$ & $-0.08(-0.14--0.02)$ & 0.012 \\
\hline Negative schizotypy & $0.10(0.04-0.16)$ & 0.001 & & \\
\hline \multicolumn{5}{|l|}{ Neglect } \\
\hline \multicolumn{5}{|l|}{ Patients } \\
\hline Positive symptoms & $0.25(0.14-0.35)$ & $<0.001$ & $0.003(-0.11-0.12)$ & 0.952 \\
\hline Negative symptoms & $0.25(0.14-0.36)$ & $<0.001$ & & \\
\hline \multicolumn{5}{|l|}{ Siblings } \\
\hline Positive schizotypy & $0.11(0.07-0.16)$ & $<0.001$ & $-0.0003(-0.04-0.04)$ & 0.991 \\
\hline Negative schizotypy & $0.11(0.07-0.16)$ & $<0.001$ & & \\
\hline \multicolumn{5}{|l|}{ Controls } \\
\hline Positive schizotypy & $0.16(0.10-0.21)$ & $<0.001$ & $-0.03(-0.10-0.03)$ & 0.277 \\
\hline Negative schizotypy & $0.12(0.06-0.18)$ & $<0.001$ & & \\
\hline
\end{tabular}

Note: PANSS, Positive and Negative Syndrome Scale; SIS-R, Structured Interview for Schizotypy - Revised;

$\mathrm{Cl}$, Confidence Interval

A priori corrected for age, gender and cannabis use

A Negative effect size indicates stronger association of trauma with positive symptoms. Positive effect size indicates stronger association of trauma with negative symptoms.

\section{Childhood trauma as a risk factor for developing psychosis or depression}

In the patient sample (including the Maastricht sample), total trauma, abuse and neglect were associated with depressive symptoms (table 5). As in the replication analysis, abuse showed a stronger association with positive than with negative symptoms; however there were no differences in influence of abuse between positive and depressive symptoms. Abuse was more strongly associated with depression than with negative symptoms. Neglect did not show any specificity for symptoms; there were no differences in influence of neglect between all three symptom clusters. 
In the sibling group (including the Maastricht sample), total trauma, abuse and neglect were also associated with depressive symptoms. Abuse showed the strongest association with depression, a weaker (yet significant) association with positive schizotypy, and the weakest (yet significant) association with negative schizotypy. Neglect showed a stronger association with depressive symptoms than with negative schizotypy. There was no evidence for a differential impact of neglect for positive versus negative schizotypy, or positive schizotypy versus depression (table 5).

In the controls (including the Maastricht sample), abuse and neglect showed the strongest association with depression, a weaker (yet significant) association with positive schizotypy, and the weakest (yet significant) association with negative schizotypy (table 5). These results were not significantly altered after adding ethnicity, family history of depression or family history of psychosis as confounders.

Table 5 Associations of trauma and depressive symptoms, PANSS symptoms (patients) or SIS-R schizotypy (siblings and healthy controls)

\begin{tabular}{|c|c|c|c|}
\hline \multirow[b]{2}{*}{ Type of childhood trauma and group } & \multirow[b]{2}{*}{ B coefficient ( $95 \% \mathrm{Cl})$} & \multicolumn{2}{|l|}{ Comparison of effect sizes ${ }^{A}$} \\
\hline & & $\begin{array}{l}\text { versus negative } \\
\text { (symptoms / schizotypy) }\end{array}$ & $\begin{array}{l}\text { versus depressive } \\
\text { symptoms }\end{array}$ \\
\hline \multicolumn{4}{|l|}{ Total trauma } \\
\hline \multicolumn{4}{|l|}{ Patients } \\
\hline Positive symptoms & $0.25^{* * *}(0.15-0.35)$ & $-0.10(-0.22-0.02)$ & $-0.06(-0.19-0.06)$ \\
\hline Negative symptoms & $0.14 * *(0.04-0.24)$ & & $-0.04(-0.16-0.08)$ \\
\hline Depressive symptoms & $0.18 * * *(0.08-0.28)$ & & \\
\hline \multicolumn{4}{|l|}{ Siblings } \\
\hline Positive schizotypy & $0.18 * * *(0.13-0.22)$ & $-0.09 * * *(-0.13--0.04)$ & $0.04(-0.01-0.08)$ \\
\hline Negative schizotypy & $0.09 * * *(0.04-0.14)$ & & $-0.13^{* * *}(-0.17--0.08)$ \\
\hline Depressive symptoms & $0.22 * * *(0.17-0.26)$ & & \\
\hline \multicolumn{4}{|l|}{ Controls } \\
\hline Positive schizotypy & $0.18 * * *(0.12-0.23)$ & $-0.10 * *(-0.16--0.04)$ & $0.09 * *(0.04-0.15)$ \\
\hline Negative schizotypy & $0.08 * *(0.02-0.13)$ & & $-0.19 * * *(-0.25--0.13)$ \\
\hline Depressive symptoms & $0.27 * * *(0.21-0.32)$ & & \\
\hline
\end{tabular}


Table 5 Continued

\begin{tabular}{|c|c|c|c|}
\hline \multirow[b]{2}{*}{ Type of childhood trauma and group } & \multirow[b]{2}{*}{ B coefficient ( $95 \% \mathrm{Cl})$} & \multicolumn{2}{|l|}{ Comparison of effect sizes ${ }^{A}$} \\
\hline & & $\begin{array}{l}\text { versus negative } \\
\text { (symptoms / schizotypy) }\end{array}$ & $\begin{array}{l}\text { versus depressive } \\
\text { symptoms }\end{array}$ \\
\hline \multicolumn{4}{|l|}{ Abuse } \\
\hline \multicolumn{4}{|l|}{ Patients } \\
\hline Positive symptoms & $0.30 * * *(0.19-0.39)$ & $-0.24 * * *(-0.36--0.12)$ & $-0.07(-0.19-0.06)$ \\
\hline Negative symptoms & $0.05(-0.05-0.15)$ & & $-0.18 * *(-0.30--0.05)$ \\
\hline Depressive symptoms & $0.23 * * *(0.12-0.33)$ & & \\
\hline \multicolumn{4}{|l|}{ Siblings } \\
\hline Positive schizotypy & $0.17 * * *(0.12-0.20)$ & $-0.08 * *(-0.12--0.03)$ & $0.09 * *(0.04-0.13)$ \\
\hline Negative schizotypy & $0.09 * * *(0.05-0.13)$ & & $-0.16 * * *(-0.21--0.12)$ \\
\hline Depressive symptoms & $0.25 * * *(0.21-0.30)$ & & \\
\hline \multicolumn{4}{|l|}{ Controls } \\
\hline Positive schizotypy & $0.18 * * *(0.12-0.23)$ & $-0.10 * * *(-0.16--0.05)$ & $0.06 *(0.004-0.12)$ \\
\hline Negative schizotypy & $0.07 * *(0.02-0.13)$ & & $-0.16 * * *(-0.22--0.11)$ \\
\hline Depressive symptoms & $0.24 * * *(0.18-0.29)$ & & \\
\hline \multicolumn{4}{|l|}{ Neglect } \\
\hline \multicolumn{4}{|l|}{ Patients } \\
\hline Positive symptoms & $0.16 * *(0.06-0.26)$ & $0.04(-0.08-0.16)$ & $-0.01(-0.14-0.11)$ \\
\hline Negative symptoms & $0.20 * * *(0.10-0.30)$ & & $0.05(-0.07-0.17)$ \\
\hline Depressive symptoms & $0.15^{* *}(0.05-0.25)$ & & \\
\hline \multicolumn{4}{|l|}{ Siblings } \\
\hline Positive schizotypy & $0.13^{* * *}(0.09-0.18)$ & $-0.03(-0.08-0.01)$ & $0.04(-0.01-0.08)$ \\
\hline Negative schizotypy & $0.10 * * *(0.06-0.15)$ & & $-0.07^{* *}(-0.11--0.02)$ \\
\hline Depressive symptoms & $0.17 * * *(0.13-0.22)$ & & \\
\hline \multicolumn{4}{|l|}{ Controls } \\
\hline Positive schizotypy & $0.17 * * *(0.11-0.22)$ & $-0.08 * *(-0.13--0.02)$ & $0.07 *(0.01-0.12)$ \\
\hline Negative schizotypy & $0.09 * *(0.03-0.14)$ & & $-0.14 * * *(-0.20--0.09)$ \\
\hline Depressive symptoms & $0.23 * * *(0.18-0.29)$ & & \\
\hline
\end{tabular}

${ }^{*} \mathrm{p}<0.05,{ }^{*} \mathrm{p}<0.01, * * * \mathrm{p}<0.001$, A priori corrected for age, gender, and cannabis

A Comparison of effect sizes.

Left column: positive versus negative symptoms/schizotypy. Negative effect sizes indicate stronger associations of trauma and positive symptoms; Right column, upper line: positive symptoms/schizotypy versus depressive symptoms. Negative effect sizes indicate stronger associations of trauma and positive symptoms; Right column, lower line: negative symptoms/schizotypy versus depressive symptoms. Negative effect sizes indicate stronger associations of trauma and depressive symptoms. 


\section{Type of childhood trauma}

Assessing the differential impact of abuse and neglect on symptoms, abuse showed a stronger association with positive symptoms than neglect, but only in the patient group (B 0.24, $95 \% \mathrm{Cl} 0.09-0.40, \mathrm{p}=0.002$ ). In the sibling group, abuse showed a stronger association with depression than neglect ( $\mathrm{B} 0.12,95 \% \mathrm{Cl} 0.03-0.22, \mathrm{p}=0.012$ ). In controls there was no differential effect of trauma type on any symptom domain. Similar results were obtained after adding ethnicity, family history of depression, and family history of psychosis as confounders.

\section{Influence of childhood trauma on course of symptoms}

Neither abuse nor neglect was significantly associated with a differential course over the 3-year follow-up period of positive, negative, or depressive symptom domains in the three groups. That is, the course of symptom domains was similar in traumatized versus nontraumatized individuals (Table 6). Adding ethnicity, family history of depression, or family history of psychosis did not influence these results significantly.

Table 6 Interaction of trauma and developmental 3-year course of PANSS symptoms (patients) and SIS-R schizotypy (siblings and controls)

\begin{tabular}{|c|c|c|c|c|c|c|}
\hline Type of symptoms and group & $\begin{array}{l}\text { Total trauma } \\
\text { B coefficient } \\
(95 \% \mathrm{Cl})\end{array}$ & $\mathbf{p}$ & $\begin{array}{l}\text { Abuse } \\
\text { B coefficient (95\% } \\
\text { Cl) }\end{array}$ & $\mathbf{p}$ & $\begin{array}{l}\text { Neglect } \\
\text { B coefficient (95\% } \\
\text { Cl) }\end{array}$ & $\mathbf{p}$ \\
\hline \multicolumn{7}{|l|}{ Patients } \\
\hline Positive symptoms & $-0.11(-0.24-0.02)$ & 0.099 & $-0.10(-0.23-0.03)$ & 0.124 & $-0.09(-0.22-0.04)$ & 0.178 \\
\hline Negative symptoms & $0.06(-0.12-0.24)$ & 0.539 & $0.04(-0.14-0.22)$ & 0.674 & $0.10(-0.08-0.28)$ & 0.297 \\
\hline \multicolumn{7}{|l|}{ Siblings } \\
\hline Positive schizotypy & $0.02(-0.03-0.08)$ & 0.403 & $0.03(-0.03-0.08)$ & 0.354 & $0.03(-0.03-0.08)$ & 0.355 \\
\hline Negative schizotypy & $0.03(-0.05-0.11)$ & 0.515 & $\begin{array}{l}-0.004 \\
(-0.08-0.07)\end{array}$ & 0.911 & $0.01(-0.07-0.09)$ & 0.802 \\
\hline \multicolumn{7}{|l|}{ Controls } \\
\hline Positive schizotypy & $-0.01(-0.09-0.06)$ & 0.787 & $-0.01(-0.08-0.06)$ & 0.788 & $\begin{array}{l}-0.001 \\
(-0.07-0.07)\end{array}$ & 0.989 \\
\hline Negative schizotypy & $0.06(-0.05-0.16)$ & 0.296 & $0.04(-0.06-0.14)$ & 0.461 & $0.01(-0.09-0.11)$ & 0.882 \\
\hline
\end{tabular}

Note: $\mathrm{Cl}$, Confidence Interval; A priori corrected for age, gender and cannabis use 


\section{DISCUSSION}

\section{Replication analyses}

The current study adds evidence to the notion that childhood trauma is associated with (subthreshold) psychosis. Evidence for a dose-response relationship was found for casecontrol, case-sibling and sibling-control comparisons, holding that more childhood trauma was associated with more severe psychopathology. These findings are in keeping with the results of Heins and colleagues ${ }^{5}$, and are now replicated by making use of a substantially larger, independent sample of patients, siblings and control subjects.

In line with recent literature we found that people who experienced abuse and neglect in their childhood are more vulnerable to develop both (subtreshold) positive and negative symptoms than people who did not experience childhood trauma $5,17,46$. Moreover, our results indicate that childhood abuse or neglect may differentially impact on symptomatology. Although associations with abuse were more pronounced for positive symptoms, associations with neglect were comparable for both symptom domains.

\section{Childhood trauma as a risk factor for developing psychosis or depression}

Our results do not support the hypothesis that childhood trauma specifically increases the chance of developing psychotic symptoms compared to depressive symptoms, not even in subjects with a (genetic risk of) psychosis. In patients with a history of childhood trauma, the risk of reporting more severe psychotic or depressive symptoms was comparable, in siblings and controls, however, stronger associations were found between trauma and depressive symptomatology. These findings indicate that childhood trauma may contribute to a shared vulnerability for psychotic and depressive symptoms.

There are several theories that could explain this shared vulnerability. One of these theories underscores the role of negative beliefs about self and others in the relationship between childhood trauma and psychopathology. In this theory, it is argued that the experience of childhood trauma may heighten the change of developing negative schemas of the self and the world ${ }^{47}$. Although negative schemata of the self and others may arguably cause depressive symptoms, Garety and colleagues showed that they could eventually also contribute to the development of psychotic symptoms ${ }^{47}$. It may be that childhood abuse or neglect increases hypervigilance to hostile cues from people in their environment; a mechanism that could also feed paranoid ideation and ideas of reference ${ }^{3,47-49}$. A second theory suggests that traumatic events alter brain systems $\mathrm{s}^{50}$ that give rise to a variety of psychiatric disorders ${ }^{51-53}$. For example, trauma during childhood could lead to a dysregulation of the hypothalamic-pituitary-adrenal (HPA) axis ${ }^{54}$, possibly caused by alterations in the cortisol feedback loop ${ }^{55,56}$, that may result in the emergence of both psychotic and/or depressive symptoms ${ }^{52,53}$. 


\section{Type of childhood trauma}

In agreement with previous work, associations between childhood abuse and symptoms were stronger than between symptoms and neglect. In addition, while associations with abuse were more pronounced for positive symptoms, associations with neglect were comparable for both symptom domains. This divergence may suggest that abuse and neglect impact differently on neurodevelopmental, social and emotional development ${ }^{2}$, 50. Abuse in particular is considered to be highly stressful and is assumed to alter brain systems that are involved in mediating the stress-response which have mostly been related to the development of positive psychotic symptoms ${ }^{52}$. A different pathway has been proposed for people with a history of neglect. This hypothesized pathway is based on the encountered association between a history of deprivation from stimulating experiences and several cognitive and psychosocial deficits in children ${ }^{57}$; deficits that in turn have been found to be associated with higher levels of both positive and negative symptoms s8,59. $^{5}$.

\section{Course of symptoms}

Our results are in agreement with general population studies suggesting that childhood trauma not only predicts the development of psychotic symptoms but also impacts on the persistency of symptoms ${ }^{9-12,14}$. In our sample, individuals with childhood trauma reported higher levels of symptoms both at baseline and 3-year follow-up in comparison to individuals without childhood trauma. This finding indicates not only that the experience of childhood trauma creates a vulnerability to develop more severe (subthreshold) psychotic symptoms, but also that these heightened symptom levels are present over time. As childhood trauma has been suggested to create enduring cognitive biases and long-lasting alterations in stress-systems associated with the development of psychotic symptoms $3,47,50,52,55,56$, one might expect that in individuals with a history of childhood trauma the severity of symptoms would have been constant or increasing over time. However, we did not find childhood trauma to be related to a differential course of symptom domains (that is, although patients with childhood trauma have higher symptom levels at baseline and follow-up, symptoms in the trauma group decreased to a similar

extent compared to the non-trauma group). This finding tentatively suggests that having experienced a traumatic event in childhood does not necessarily indicate a deteriorating outcome compared to those who did not experienced a traumatic event in childhood, at least not on symptom level.

\section{Methodological issues}

Some methodological limitations need to be taken into consideration when interpreting these findings. First, childhood trauma was measured by using a retrospective, self-report questionnaire, which increases the chance of report(ing) biases and recall biases.

However, studies on retrospective self-report measures of childhood trauma have shown 
considerable reliability ${ }^{60}$. Moreover, the instrument we used to measure childhood trauma is well-validated and was found to be a reliable measurement in previous studies ${ }^{31,32}$.

Second, the study lacks detailed information about the experienced childhood trauma, such as timing, age of occurrence, subject's relationship to the perpetrator, severity and duration of trauma. Morgan and Fisher argued that when investigating trauma, timing, (perceived) severity, and duration of trauma should be taken into account because they are likely to influence the association with psychotic symptomatology ${ }^{61}$. This is considered an important issue for further research on childhood trauma.

Third, for the measurement of depressive symptoms in different groups of participants different instruments were used. However, the CDS was specifically developed for the reliable and valid assessment of depression in schizophrenia patients, in contrast to the CAPE, which is developed for assessing positive, negative, and depressive symptoms in nonclinical groups. In particular, the CAPE is less sensitive to differentiate between negative and depressive symptoms. Therefore, we preferred to use the CDS for our within group analyses assessing the different influence of childhood trauma on positive, negative, and depressive symptoms. However, as a limitation we cannot make inferences about the differential influence of childhood trauma on symptomatology between these groups. Fourth, although we made an effort to include a representative prevalence sample of patients with psychosis and their siblings in the present study, we cannot fully exclude the possibility of selection bias. Unfortunately we have no systematic records of reasons for refusal of all eligible cases that were approached but refused participation. This should be taken into account when considering the generalizability of our findings. Moreover, we were not able to prevent drop-out of participants over time. We found those participants who were lost during follow-up were more often non-white compared with participants completing both measurements. Controls who did participate on both measurements were slightly older than controls not participating on both measurements. We have investigated whether ethnicity (white / non-white), gender and sex were influential confounders for our analyses, and found that they were not. Therefore we do not believe that the drop-out of participants over time have a great impact on our results.

The major strength of this study is that we were able to take into account the association with the course of symptom domains and the association with depression in subjects with variation in psychosis vulnerability. By using special statistical techniques, we were able to assess the influence of trauma on several different, yet often co-occurring symptoms.

In conclusion, this study strengthens and extends the evidence for a robust association between childhood trauma and psychotic symptoms across different levels of severity. However, we found no support for the hypothesis that childhood trauma specifically increases the chance of developing psychotic symptoms compared to depressive symptoms. Moreover, although patients with childhood trauma have higher symptom levels at baseline and at the 3-year follow-up, childhood trauma was not associated with a differential course of symptoms. 


\section{Acknowledgements}

We are grateful for the generosity of time and effort by the families who make the GROUP project possible.

\section{Financial support}

This work was supported by the Geestkracht program of the Dutch Health Research Council (ZON-MW, grant number 10-000-1002) and matching funds from participating universities and mental health care organizations (Site Amsterdam: Academic Psychiatric Centre AMC, Ingeest, Arkin, Dijk en Duin, Rivierduinen, Erasmus MC, GGZ Noord Holland Noord; Site Utrecht: University Medical Centre Utrecht, Altrecht, Symfora, Meerkanten, Riagg Amersfoort, Delta; Site Groningen: University Medical Centre Groningen, Lentis, GGZ Friesland,GGZ Drenthe, Dimence, Mediant, GGZ De Grote Rivieren and Parnassia psychomedical centre; Site Maastricht: Maastricht University Medical Centre, GGZ Eindhoven, GGZ Midden-Brabant, GGZ Oost-Brabant, GGZ Noord- Midden Limburg, Mondriaan Zorggroep, Prins Clauscentrum Sittard, RIAGG Roermond, Universitair Centrum Sint-Jozef Kortenberg, CAPRI University of Antwerp, PC Ziekeren Sint-Truiden, PZ Sancta Maria Sint-Truiden, GGZ Overpelt, OPZ Rekem). The analyses were supported by unrestricted grants from JansenCilag, Eli Lilly and Company, Astra-Zeneca and Lundbeck. The research leading to these results has received funding from the European Community's Seventh Framework Program under grant agreement No. HEALTH-F2-2009-241909 (Project EU-GEI). 


\section{REFERENCES}

1. Varese F, Smeets F, Drukker M, et al. Childhood adversities increase the risk of psychosis: a meta-analysis of patient-control, prospective- and cross-sectional cohort studies. Schizophr Bull 2012;38(4):661-671.

2. Glaser D. Child abuse and neglect and the brain--a review. J child psychol psychiat, allied disc Jan 2000;41(1):97-116.

3. Bentall R, Fernyhough C. Social predictors of psychotic experiences: specificity and psychological mechanisms. Schizophr Bull 2008;34:1009-1011.

4. Bentall R, Wickham S, Shevlin M, Varese F. Do specific early-life adversities lead to specific symptoms of psychosis? A study from the 2007 the adult psychiatric morbidity survey. Schizophr Bull 2012;38(4):734-740.

5. Heins $M$, Simons $C$, Lataster $T$, et al. Childhood trauma and psychosis: A case-control and case-sibling comparison across different levels of genetic liability, psychopathology, and type of trauma. Am J Psychiat 2011;168(12):1286-1294.

6. Hox JJ. Multilevel analysis: techniques and applications. 2nd ed. New York, NY: Routledge; 2010.

7. Matheson SL, Shepherd AM, Pinchbeck RM, Laurens KR, Carr VJ. Childhood adversity in schizophrenia: a systematic meta-analysis. Psychol Med 2012;43(2):225-238.

8. Cougnard A, Marcelis M, MyinGermeys I, et al. Does normal development expression of psychosis combine with environmental risk to cause persistence of psychosis? A psychosis proneness-persistence model. Psychol Med 2007;

37:513-527.
9. de Loore E, Drukker M, Gunther N, et al. Childhood negative experiences and subclinical psychosis in adolescence: a longitudinal general population study. Early Int Psychiatry 2007;1:201-207.

10. Schreier A, Wolke $D$, Thomas K, et al. Prospective study of peer victimization in childhood and psychotic symptoms in a nonclinical population at age 12 years. Arch Gen Psychiat 2009;66(5):527-536.

11. Arseneault $L$, Cannon $M$, Fisher $H$, Polanczyk G, Moffitt TE, Caspi A. Childhood trauma and children's emerging psychotic symptoms: a genetically sensitive longitudinal cohort study. Am J Psychiat 2011;168:65-72.

12. Mackie CJ, Castellanos-Ryan N, Conrod PJ. Developmental trajectories of psychotic-like experiences across adolescence: impact of victimization and substance use. Psychol Med 2011;41:47-58.

13. Wigman JT, van Winkel $R$, Raaijmakers QA, Ormel J, Verhulst FC, Reijneveld $\mathrm{SA}$, van Os J, Vollebergh WA. Evidence for a persistent, environment-dependent and deteriorating subtype of subclinical psychotic experiences: a 6-year longitudinal general population study. Psychol Med 2011;41(11):2317-2329.

14. Kelleher I, Keeley H, Corcoran P, et al. Childhood trauma and psychosis in a prospective cohort study: cause, effect, and directionality. Am J Psychiat 2013;170(7):734-741.

15. Greenfield SF, Strakowski SM, Tohen $\mathrm{M}$, Batson SC, Kolbrener ML. Childhood abuse in first-episode psychosis. Br J Psychiat Jun 1994;164(6):831-834. 
16. Lysaker PH, Beattie NL, Strasburger AM, Davis LW. Reported history of child sexual abuse in schizophrenia: associations with heightened symptom levels and poorer participation over four months in vocational rehabilitation. J Nerv Ment Dis Dec 2005;193(12):790-795.

17. Ross CA, Anderson G, Clark P. Childhood abuse and the positive symptoms of schizophrenia. Hosp Comm Psychiat 1994;45:489-491.

18. Conus $P$, Cotton $S$, Schimmelmann BG, Berk M, Daglas R, McGorry PD, Lambert M. Pretreatment and outcome correlates of past sexual and physical trauma in 118 bipolar I disorder patients with a first episode of psychotic mania. Bipolar Disord May 2010;12(3):244-252.

19. Leverich GS, McElroy SL, Suppes $T$, et al. Early physical and sexual abuse associated with an adverse course of bipolar illness. Biol Psychiat Feb 15 2002;51(4):288-297.

20. Neria Y, Bromet EJ, Carlson GA, Naz B. Assaultive trauma and illness course in psychotic bipolar disorder: findings from the Suffolk county mental health project. Acta Psychiatr Scand May 2005;111(5):380-383.

21. Zlotnick C, Ryan CE, Miller IW, Keitner Gl. Childhood abuse and recovery from major depression. Child Abuse Negl Dec 1995;19(12):1513-1516.

22. Hovens JGFM, Wiersma JE, Spinhoven P, Penninx BWJH, Zitman FG. Impact of childhood life events on the course of depressive and anxiety disorders. Acta Psychiatr Scand 2012;DOI: 10.1111/j.1600-0447.2011.01828.x.

23. Korver N, Quee PJ, Boos HB, Simons C, de Haan L. Genetic Risk and
Outcome of Psychosis (GROUP), a multi site longitudinal cohort study focused on gene-environment interaction: objectives, sample characteristics, recruitment and assessment methods. Int J Meth Psychiat Res 2012;21(3):205-221.

24. Wechsler D. WAIS-III: Wechsler Adult Intelligence Scale (3rd edition). Administration and Scoring Manual. San Antonio, TX: Psychological Corporation; 1997.

25. Maxwell ME. Manual for the FIGS (Family Interview for Genetic Studies). Bethesda, MD: Clinical Neurogenetics Branch, Intramural Research Program, National Institute of Mental Health; 1992.

26. Andreasen NC, Flaum M, Arndt S. The comprehensive assessment of symptoms and history (CASH). An instrument for assessing diagnosis and psychopathology. Arch Gen Psychiat 1992;49(8):615-623.

27. Wing JK, Babor $\mathrm{T}$, Brugha $\mathrm{T}$, et al. SCAN. Schedules for Clinical Assessment in Neuropsychiatry. Arch Gen Psychiat 1990;47(6):589-593.

28. Andreasen NC. Scale for the assessment of positive symptoms. Iowa City, IA: University of lowa; 1984.

29. Andreasen NC. Negative symptoms in schizophrenia. Definition and reliability. Arch Gen Psychiat Jul 1982;39(7):784-788.

30. A.P.A. Diagnostic and Statistical Manual of Mental Disorders (DSM-IV$T R$ ), 4th edition. Washington, DC: American Psychiatric Association; 2000.

31. Bernstein DP, Stein JA, Newcomb MD, et al. Development and validation of a brief screening version of the 
Childhood Trauma Questionnaire. Child Ab Neg/ 2003;27:169-190.

32. Thombs BD, Bernstein DP, Lobbestael J, Arntz A. A validation study of the Dutch Childhood Trauma Questionnaire-Short Form: factor structure, reliability, and knowngroups validity. Child Ab Negl Aug 2009;33(8):518-523.

33. Kay SR, Fiszbein A, Opler LA. The positive and negative syndrome scale (PANSS) for schizophrenia. Schizophr Bull 1987;13(2):261-276.

34. Kendler KS, Lieberman JA, Walsh D. The Structured Interview for Schizotypy (SIS): a preliminary report. Schizophr Bull 1989;15(4):559-571.

35. Vollema MG, Ormel J. The reliability of the structured interview for schizotypy-revised. Schizophr Bull 2000;26(3):619-629.

36. Vollema MG, Postma B.

Neurocognitive correlates of schizotypy in first degree relatives of schizophrenia patients. Schizophr Bull 2002;28(3):367-377.

37. Hanssen $\mathrm{M}$, Krabbendam L, Vollema $M$, Delespaul P, Van Os J. Evidence for instrument and family-specific variation of subclinical psychosis dimensions in the general population. $J$ Abnorm Psychol Feb 2006;115(1): 5-14.

38. van Nierop M, Van Os J, Gunther N, et al. Phenotypically continuous with clinical psychosis, discontinuous in need for care: Evidence for an extended psychosis phenotype. Schizophr Bull 2012;38(2):231-238.

39. Addington $D$, Addington J, Schissel B. A depression rating scale for schizophrenics. Schizophr Res Jul-Aug 1990;3(4):247-251.
40. WHO. Composite International Diagnostic Interview (CIDI), Version 1.0. Geneva, Switzerland: World Health Organization; 1990.

41. van Winkel $R$, Genetic Risk and Outcome of Psychosis (GROUP) investigators. Family-based analysis of genetic variation underlying psychosis-inducing effects of cannabis. Arch Gen Psychiat 2011;68(2): 148-157.

42. Matheson SL, Shepherd AM, Laurens $\mathrm{KR}$, Carr VJ. A systematic meta-review grading the evidence for non-genetic risk factors and putative antecedents of schizophrenia. Schizophr Res Dec 2011;133(1-3):133-142.

43. Morgan $C$, Charalambides $M$, Hutchinson G, Murray RM. Migration, ethnicity, and psychosis: toward a sociodevelopmental model. Schizophr Bull 2010;36(4):655-664.

44. Van Winkel R, Van Nierop M, MyinGermeys I, van Os J. Childhood Trauma as a Cause of Psychosis: Linking Genes, Psychology, and Biology. Can J Psychiat 2013;58(1): 44-51.

45. Pocock SJ, Assmann SE, Enos LE, Kasten LE. Subgroup analysis, covariate adjustment and baseline comparisons in clinical trial reporting: current practice and problems. Stat Med 2002;21(19):2917-2930.

46. Janssen I, Krabbendam L, Bak M, Hanssen M, Vollebergh W, De Graaf R, Van Os J. Childhood abuse as a risk factor for psychotic experiences. Acta Psychiat Scand 2004;109:38-45.

47. Garety PA, Kuipers E, Fowler D, Freeman D, Bebbington PE. A cognitive model of the positive symptoms of 
psychosis. Psychol Med Feb

2001;31(2):189-195.

48. Morrison AP, Frame L, Larkin $W$.

Relationships between trauma and

psychosis: a review and integration.

Br J Clin Psychol Nov 2003;42(Pt 4):

331-353.

49. Read J, Gumley A. Can attachment theory help explain the relationship between childhood adversity and psychosis? Attachment: New Dir Psychother Rel Psychoan 2008;2:1-35.

50. Perry BD. Child maltreatment: $a$ neurodevelopmental perspective on the role of trauma and neglect in psychopathology in Child and Adolescent Psychopathology. Somerset, NJ: John Wiley \& Sons; 2008.

51. Heim C, Nemeroff CB. The role of childhood trauma in the neurobiology of mood and anxiety disorders: preclinical and clinical studies. Biol Psychiatry Jun 15 2001; 49(12): 1023-1039.

52. Kapur S. Psychosis as a state of aberrant salience: a framework linking biology, phenomenology, and pharmacology in schizophrenia.

Am J Psychiat 2003;160:13-23.

53. Heim C, Newport DJ, Mletzko T, Miller $\mathrm{AH}$, Nemeroff CB. The link between childhood trauma and depression: insights from HPA axis studies in humans. Psychoneuroendocrinology Jul 2008;33(6):693-710.

54. Walker EF, Diforio D. Schizophrenia: a neural diathesis-stress model. Psychol Rev Oct 1997;104(4):667-685.

55. Binder EB, Bradley RG, Liu W, et al. Associations of FKBP5 polymorphisms and childhood abuse with risk of posttraumatic stress disorder symp- toms in adults. JAMA

2008;299(11):1291-1305.

56. Collip D, Myin-Germeys I, Wichers M, et al. FKBP5 as a possible moderator of the psychosis-inducing effects of childhood trauma. $B$ J Psychiat 2013;202(4):261-268.

57. Colvert E, Rutter M, Kreppner J, et al. Do theory of mind and executive functioning deficits underlie the adverse outcomes associated with profound early deprivation? Findings from the English and Romanian adoptees study. J Abn Child Psychol 2008;35:1057-1068.

58. Rabinowitz J, De Smedt G, Harvey PD, Davidson M. Relationship between premorbid functioning and symptom severity as assessed at first episode of psychosis. Am J Psychiat Dec 2002;159(12):2021-2026.

59. Addington J, van Mastrigt $S$, Addington D. Patterns of premorbid functioning in first-episode psychosis: initial presentation. Schizophr Res Jul 1 2003;62(1-2):23-30.

60. Fisher HL, Craig TK, Fearon P, et al. Reliability and comparability of psychosis patients' retrospective reports of childhood abuse. Schizophr Bull 2011;37(3):546-553.

61. Morgan C, Fisher H. Environmental factors in schizophrenia: childhood trauma - a critical review. Schizophr Bull 2007;33(1):3-10. 


\title{
CHAPTER 9
}

\section{Evidence that Psychotic Symptoms Are Prevalent in Disorders of Anxiety and Depression, Impacting on IIIness Onset, Risk, and Severity-Implications for Diagnosis and Ultra-High Risk Research}

\author{
Johanna Wigman ${ }^{\mathrm{AB}}$ \\ Martine van Nierop ${ }^{B}$ \\ Wilma Vollebergh ${ }^{\mathrm{A}}$ \\ Roselind Lieb ${ }^{\mathrm{CD}}$ \\ Katja Beesdo-Baum ${ }^{\mathrm{c}}$ \\ Hans-Ullrich Wittchen ${ }^{\mathrm{CE}}$ \\ Jim van Os ${ }^{\mathrm{BF}}$
}

\footnotetext{
A Department of Interdisciplinary Social Science, University of Utrecht, 3508 TC Utrecht, The Netherlands

${ }^{B}$ Department of Psychiatry and Psychology, School of Mental Health and Neuroscience, Maastricht University Medical Center, PO Box 616, 6200 MD Maastricht, The Netherlands

c Clinical Psychology and Epidemiology Unit, Max Planck Institute of Psychiatry, Munich, Germany

${ }^{D}$ Department of Epidemiology and Health Psychology, Institute of Psychology, University of Basel, Basel, Switzerland

E Institute of Clinical Psychology and Psychotherapy, Technical University Dresden, Dresden, Germany

F Department of Psychosis Studies, King's College London, King's Health Partners, Institute of Psychiatry, De Crespigny Park, London, UK
} 


\section{ABSTRACT}

\section{Background}

It is commonly assumed that there are clear lines of demarcation between anxiety and depressive disorders on the one hand, and psychosis on the other. Recent evidence, however, suggests that this principle may be in need of updating.

\section{Methods}

Depressive and/or anxiety disorders, with no previous history of psychotic disorder, were examined for the presence of psychotic symptoms in a representative community sample of adolescents and young adults (Early Developmental Stages of Psychopathology study; $\mathrm{n}=3021$ ). Associations and consequences of psychotic symptomatology in the course of these disorders were examined in terms of demographic distribution, illness severity, onset of service use, and risk factors.

\section{Results}

Around $27 \%$ of those with disorders of anxiety and depression displayed one or more psychotic symptoms, vs $14 \%$ in those without these disorders (OR 2.23, 95\% Cl 1.89-2.66, $\mathrm{p}<0.001)$. Presence as compared with nonpresence of psychotic symptomatology was associated with younger age $(p<0.0001)$, male sex $(p<0.0058)$, and poorer illness course $(p<0.0002)$. In addition, there was greater persistence of schizotypal $(p<0.0001)$ and negative symptoms $(p<0.0170)$, more observable illness behaviour $(p<0.0001)$, greater likelihood of service use $(p<0.0069)$, as well as more evidence of familial liability for mental illness $(p<0.0100)$, exposure to trauma $(p<0.0150)$, recent and more distant life events $(p<0.0006-0.0244)$, cannabis use $(p<0.0009)$, and any drug use $(p<0.0008)$.

\section{Conclusion}

Copresence of psychotic symptomatology in disorders of anxiety and depression is common and a functionally and etiologically highly relevant feature, reinforcing the view that psychopathology is represented by a network of overlapping and reciprocally impacting dimensional liabilities. 


\section{INTRODUCTION}

Affective dysregulation and reality distortion are correlated but separable dimensions of psychopathology ${ }^{1}$. The association is present over the continuum of subclinical and clinical expression of psychopathology, although stronger in the clinical range ${ }^{2}$. Symptoms and syndromes of depression and anxiety ${ }^{3,4}$ are present in the majority of patients with schizophrenia, and these affective symptoms may distinguish distinct subgroups within clinical samples of individuals with psychotic illness 5 . Although the combination of affective disorder with superimposed psychotic disorder is considered rare $^{6}$, psychotic symptoms are often reported in patients with affective disorders ${ }^{7,8}$. Interestingly, the great majority of help-seeking individuals meeting ultra-high risk criteria (UHR) for psychotic disorder in fact initially present with anxiety disorder or major depression ${ }^{9-11}$, and the same is reported in individuals at psychometric risk for psychosis ${ }^{12}$. Epidemiological community and general population studies have furthermore reported strong associations between the subclinical expression of affective and psychotic symptoms ${ }^{13,14}$.

Thus, affective dysregulation (anxiety and depression) and reality distortion are coexpressed across the range of subclinical and clinical expression. In part, this may be considered the result of mental states that causally impact on each other, eg, affective dysregulation giving rise to psychotic symptoms ${ }^{15-17}$. In addition, genetic studies have suggested familial links between affective and psychotic disorders ${ }^{18,19}$ as well as between schizophrenia and a range of other mental disorders in the nonpsychotic spectrum ${ }^{20}$, suggesting shared liabilities. Furthermore, dimensions of affective and psychotic pathology are associated with similar risk factors ${ }^{19,}{ }^{21}$, although quantitative differences exist in strength of association. There is also evidence of shared underlying endophenotypes such as alterations in cognition ${ }^{19,22}$, as well as social and emotional functioning ${ }^{6,19}$. Additional support for a common factor underlying both affective and psychotic pathology, or for reciprocal causal influence, comes from longitudinal studies showing that subclinical psychotic experiences predict not only later onset of psychotic disorders ${ }^{23}$ but also later affective disorders ${ }^{24}$, even when the psychotic experiences are not considered clinically relevant ${ }^{25}$.

Evidence suggests that the predictive value of either psychotic or affective symptoms for later psychopathology and worse outcome is highest when they co-occur. One study showed that copresence of subclinical manic and psychotic experiences predicted the development of bipolar disorder more strongly that subclinical manic experiences in isolation ${ }^{26}$. Likewise, the risk of developing a psychotic disorder was highest for individuals with combined expression of subclinical psychotic and affective experiences compared with those with only expression of psychotic experiences ${ }^{27}$. Furthermore, co-occurrence of subclinical psychotic experiences predicted poorer outcome in a community sample of patients with major depressive disorder ${ }^{28}$.

In sum, affective and psychotic phenomena often co-occur, partly on the basis of shared vulnerability and partly on the basis of reciprocal causal influence; co-occurrence predicts 
poorer course and outcome. These findings have major conceptual and practical implications for diagnosis and treatment. Classificatory principles for mental disorders have been dominated by the nomothetic approach in which criteria relating to symptoms and complaints in patients are assumed to be indicators of an underlying latent diagnostic construct. This approach, however, may not be in agreement with evidence that symptoms in practice form part of overlapping and reciprocally impacting dimensional liabilities that give rise to highly patient-specific admixtures and trajectories ${ }^{29}$.

Given the above findings, it may well be that interindividual differences in dimensions that cross the boundaries of traditional disorders have been neglected to the point that important patterns of admixture impacting severity, course, and etiology have been overlooked. A case in point is the group of disorders of anxiety/depression that traditionally are not considered as fundamentally related to expression of psychosis. Instead, expression of psychosis in these disorders is reserved for the definition of rare cases where psychotic symptoms are dominant and clinically severe (as in the case of major depression with psychotic features). However, given the above suggestion of substantial shared vulnerability, shared expression, and reciprocal impact between affective dyregulation and reality distortion, the following questions suggest themselves: (1) how frequent is the expression of psychotic experiences in broadly defined disorders of anxiety and depression? and (2) does the occurrence of psychotic symptoms matter in terms of demography, onset, severity, and etiology?

\section{METHODS}

\section{Sample}

Data from the Early Developmental Stages of Psychopathology (EDSP) study were used. The EDSP study collected data on prevalence, incidence, risk factors, comorbidity, and course of mental disorders in a representative general population sample of adolescents and young adults. Detailed description of the study, sampling methods, instruments, and procedures can be found elsewhere ${ }^{30-32}$. The study was approved by the standing ethics committee. The baseline sample was drawn from population registry offices of Munich and its 29 counties in 1994. This sample was drawn to mirror the distribution of individuals expected to be 14-24 years of age at the time of the baseline (T0) interview in 1995.

The EDSP study was designed as a prospective, longitudinal study consisting of 4 data waves: baseline (TO) and 3 follow-up waves at an average of, respectively, 1.6 (TO-T1, SD 0.2), 3.5 (TOT2, SD 0.3), and 8.4 (TO-T3, SD 0.7) years after T0.

The younger participants (14-17 y) were assessed 4 times and subjects aged 18-24 years only 3 times. For the current analyses, data from waves with all participants were used (T0, T2, and T3). 


\section{Instruments}

\section{Psychopathology}

Symptoms, syndromes, and disorders were assessed with the computer-assisted version of the Munich-Composite International Diagnostic Interview (DIA-X/M-CIDI) ${ }^{33}$, an updated an expanded version of the WHO's CIDI version 1.2 (WHO 1990). The DIA-X/M-CIDI is a comprehensive, fully standardized, diagnostic interview, addressing symptoms, syndromes, and diagnoses of a wide range of mental disorders in accordance with definitions and criteria of the Diagnostic and Statistical Manual of Mental Disorders, Fourth Edition and International Classification of Diseases, Tenth Edition. The M-CIDI has been shown to be both reliable and valid. Interviews were conducted by fully trained and experienced psychologists, who were allowed to probe with follow-up questions, which is particularly relevant for the assessment of psychotic symptoms because these are sensitive to falsepositive ratings. The EDSP covers a total observation period of up to 10 years. At T0 (baseline), the lifetime version of the DIA-X/M-CIDI was used; for subsequent waves, the respective DIA-X/M-CIDI internal versions were used. Based on the relevant anxiety disorder and depression sections, dichotomous variables were constructed representing whether an individual had received (1) or had not received (0) a diagnosis of, respectively, a major depressive disorder or any anxiety disorder (includes panic disorder, General Anxiety Disorder [GAD], agoraphobia, specific phobias, and social anxiety disorder, posttraumatic stress disorder [PTSD], and Obsessive Compulsive Disorder [OCD]). The course of disorders of anxiety/depression was measured as the number of times an individual had received any diagnosis in these sections at each wave, at T0, T2, and T3 (range 0-3). Thus, a score of 3 indicates the presence of any anxiety and depressive disorder at all 3 time points. The G-section of the interview on psychotic symptoms and their clinical relevance were only collected at T2 (lifetime version) and T3 (interval version). Presence of positive psychotic symptoms was broadly defined as any rating of "present" on any of the 20 core psychosis items, as described previously ${ }^{34}$.

Based on the CIDI measures described above, a 3-level variable was constructed indicating whether an individual had (0) no affective (ie, major depressive disorder or any anxiety) disorder and no CIDI psychotic symptoms (the reference group), (1) an affective disorder but no CIDI psychotic symptoms, or (2) an affective disorder and CIDI psychotic symptoms at either T2 or T3. This variable effectively expressed lifetime coexpression of the behavioural liability to psychosis in individuals with major depressive disorder or any anxiety disorder and is hereafter referred to as "disorders of anxiety/depression". Individuals with a diagnosis of psychotic disorder at T2 or T3 (N=21) or bipolar disorder (bipolar I or bipolar II) at any time point $(\mathrm{N}=90)$ were excluded from analysis, as otherwise any differences between disorders of anxiety/depression with and without psychotic symptoms would be confounded by psychotic disorder.

Suicidal ideation was addressed with CIDI items rating whether the participant had ever had thoughts about suicide, conforming to previous work ${ }^{35}$. Item ratings were summed over the 3 assessments (range of possible values for each: 0-3). 
Negative symptoms were coded as present when the interviewer rated as present item $\mathrm{X} 11$ (on flat emotions) and/or X12 (on inadequate communication), in line with previous analyses in this sample ${ }^{34}$. Persistence of negative symptoms was calculated by scoring whether any negative symptom was present never (0) or at $1(1), 2(2)$, or all $3(3)$ time points, as described previously.

The Symptom Checklist-90-R (SCL-90), a reliable and valid screening instrument for a range of symptoms occurring in the last week, was also administered at all time points. The SCL-90 subscales on psychoticism and paranoid ideation, which rate a broader psychosis phenotype indexing the personality - or schizotypal - dimension of psychosis, were summed to create an SCL-schizotypy score at each time point. Persistence of schizotypy expression was subsequently calculated by scoring whether an individual was in the highest $10 \%$ of SCLschizotypy scores never (0) or at 1 (1), 2 (2), or all three (3) time points ${ }^{36}$. Because this schizotypy persistence score was based on the SCL-90-R and not the CIDI, it could be used to compare anxiety/depression groups without CIDI psychotic symptoms.

\section{Clinical Relevance}

\section{Help-Seeking Behaviour}

In line with previous analyses reported elsewhere ${ }^{36}$, help-seeking behaviour was defined as general help-seeking-behaviour, which was broadly defined as having visited any mental health institution ever for any mental health problem (based on the Q-section of the M-CIDI).

\section{Caseness}

Based on the X16 M-CIDI item, a variable indicating "caseness" was constructed, reflecting the interviewer's opinion on clinical evidence of mental illness in the participant, scored as not noticeable (0), slightly noticeable (1), clearly noticeable (2), and very ill (3).

Conforming to previous work ${ }^{36}$, a dichotomous variable was made based on this item indicating presence of clearly noticeable level of mental disorder (defined as score $>1$ ).

\section{Psychiatric Medication Use}

As part of a module assessing mental health treatments, participants were shown a list of different types of medication and were asked to endorse those they had been given for any psychopathological or psychosomatic problem. The acknowledgement of any psychiatric medication other than antipsychotic medication at T2 and T3 was rated and used as a binary variable in the analyses. 


\section{Risk Factors}

\section{Substance Use}

Substance use from any drug or nonprescribed medication was assessed with the L-section of the M-CIDI, assessed at all 3 time points. Conforming to previous work ${ }^{37}, 2$ variables indexing substance use were defined dichotomously as use of (1) any substance and (2) cannabis more than 5 times ever at each time point.

\section{Trauma}

Self-reported lifetime exposure to trauma was assessed using the $\mathrm{N}$-section of the M-CIDI on trauma and PTSD comprising 9 groups of specific traumatic events (presented by a respondent list) such as "experienced physical threat", "experienced serious accident", or "being sexually abused as a child". Consistent with earlier analyses ${ }^{38}$, positive responses to any of the events were coded as "self-reported trauma".

\section{Recent Life Events}

Recent life events were assessed at T2, with the Munich Interview for the Assessment of Life Events and Conditions (Münchner Ereignis Liste); a reliable 3-step interview assessing recent life events. For each of the 4 years over the period of 1995-1998, the total sum of positive and negative life events was calculated.

\section{Urbanicity}

Consistent with previous work ${ }^{39}$, urbanicity was defined as living in the urban region of the German city of Munich vs the surrounding areas of Munich. The urban area, thus defined, had a population density of 4061 persons per square mile; for the rural area, this was 553 persons per square mile.

\section{Family History of Help Seeking}

The item P8 of the TO M-CIDI, rating the proband's report on whether any of the proband's family members had ever sought help for emotional or mental problems, was used as a proxy for family liability for mental disorder. 


\section{Analyses}

All analyses were carried out with STATA 11.0. Using the MLOGIT command, multinomial logistic regression was used to predict the 3-level outcome variable of disorders of anxiety/ depression with/without psychotic symptoms. Given the fact that this outcome was measured twice (lifetime at T2 and interval T2-T3 at T3), data were analyzed in the "longformat", each individual contributing 2 observations (T2 and T3) for analysis, conform previous work ${ }^{35,36}$. In order to correct for the clustering of multiple observations within subjects, cluster-robust standard errors were computed using the CLUSTER option in the MLOGIT module in STATA. Individuals without disorders of anxiety/depression and without psychotic symptoms were the reference group. Presence of disorders of anxiety/ depression with and without psychotic symptoms was predicted by (1) demographic variables (age, gender, and education), (2) course and severity variables (course of disorders of anxiety/depression, persistence of both schizotypy expression, negative symptoms, and suicidal thoughts), (3) variables relevant for onset of professional help (help-seeking behaviour and caseness), and (4) risk factors (family history of mental disorder, trauma, life events, substance use, and urbanicity). All analyses were a priori adjusted for age, sex, and education. OR for disorders of anxiety/depression, with and without psychotic symptoms were compared by Wald test using the postestimation TEST command in STATA.

\section{Risk Set}

The risk set for analysis were individuals at T2 and T3 who (1) had no diagnosis of bipolar disorder or psychotic disorder, (2) had a diagnosis of disorders of anxiety/depression as defined above, and (3) did not present with psychotic symptoms in the absence of disorders of anxiety/depression as defined above. The reference group consisted of individuals who had neither psychotic/bipolar disorder nor anxiety/depression. This yielded a total risk set of 2118 individuals at T2 and 2027 individuals at T3.

\section{Sensitivity Analysis}

A planned sensitivity analysis was carried out excluding individuals with lifetime comorbid anxiety disorder and depressive disorder, sensitively using measures at T0, T2, and T3, in order to examine to what degree any differences between individuals with disorders of anxiety/depression with and without psychotic symptoms was mediated by comorbidity of anxiety and depression, which is associated with greater indices of illness severity and poorer prognosis ${ }^{40}$. 


\section{RESULTS}

\section{Descriptives}

Before exclusion of individuals with bipolar disorder and psychotic disorder, the number of eligible individuals at T0 was $\mathrm{N}=3021$, at T2 $\mathrm{N}=2548$ (84\%), and at T3 $\mathrm{N}=2210$ (73\%). Of all individuals with disorders of anxiety/depression, as defined for the purpose of this study, $27 \%$ also reported psychotic symptoms at any time point ( $36 \%$ at T2 and $19 \%$ at T3), vs $14 \%$ in those without (OR $2.23,95 \% \mathrm{Cl} 1.89-2.66, \mathrm{p}<0.001$ ).

\section{Associations of Demographics, Severity, Risk Factors, and Onset of Professional Help With Disorders of Anxiety/Depression With/Without Psychotic Symptoms}

Analyses in the risk set as defined above (described in Table 1) revealed that participants with a disorder of anxiety/depression with psychotic symptoms, compared with those without psychotic symptoms, were more likely to be male and younger (Table 2).

Lower education differentiated between disorders of anxiety/depression with and without psychotic symptoms, the former group having lower educational attainment. Measures of severity (persistence of both schizotypal and negative psychotic symptoms, suicidal ideation over T0-T3) and course (number of times diagnosed with disorders of anxiety/depression over T0-T3) were associated with both affective conditions but more strongly with disorders of anxiety/depression with psychotic symptoms. The same was found for associations with variables relevant for onset of professional help and risk factors: these variables were significantly associated with presence of disorders of anxiety/depression with and without psychotic symptoms but more strongly with disorders of anxiety/depression with psychotic symptoms.

The sensitivity analyses showed that results were largely robust to exclusion of comorbid anxiety and depression states from the analyses (see last column Table 2). Generally, all patterns remained similar, ie, quantitative differences were found between disorders of anxiety/depression with and without psychotic symptoms, with the strongest associations found for individuals with additional psychotic symptoms. Three predictors no longer discriminated significantly between the 2 groups, namely, use of cannabis $(p<0.0622)$, help seeking $(p<0.208)$, and life events that had occurred in the second year after baseline assessment $(p<0.068)$. However, the results still showed a trend toward quantitative differences and still displayed the strongest associations for individuals with additional psychotic symptoms. 
Table 1 Descriptives of Risk Set at T2 and T3, as well as of Entire Population at T2 and T3

\begin{tabular}{|c|c|c|c|c|}
\hline & \multicolumn{2}{|l|}{ Risk Set } & \multicolumn{2}{|c|}{ Total Sample } \\
\hline & T2 & T3 & T2 & T3 \\
\hline $\mathrm{N}$ total & 2118 & 2027 & 2548 & 2210 \\
\hline Mean age (SD) & $21.8(3.4)$ & $26.6(3.5)$ & $21.7(3.4)$ & $26.6(3.5)$ \\
\hline$\%$ Females & 49.8 & 49.7 & 49.1 & 48.6 \\
\hline \multicolumn{5}{|l|}{ Level of education } \\
\hline Low & $264(12 \%)$ & $239(12 \%)$ & $323(13 \%)$ & $271(12 \%)$ \\
\hline Medium & $647(31 \%)$ & $582(29 \%)$ & $769(30 \%)$ & $647(30 \%)$ \\
\hline High & $1207(57 \%)$ & $1206(59 \%)$ & $1456(57 \%)$ & $1292(58 \%)$ \\
\hline \multicolumn{5}{|l|}{ Major depressive episode } \\
\hline Not present & 1905 (90\%) & $1791(88 \%)$ & 2296 (90\%) & $1972(89 \%)$ \\
\hline Present & $213(10 \%)$ & $236(12 \%)$ & $233(9 \%)$ & $238(11 \%)$ \\
\hline Missing & 0 & 0 & $19(1 \%)$ & 0 \\
\hline \multicolumn{5}{|l|}{ Anxiety disorder } \\
\hline Not present & $1756(83 \%)$ & 1649 (81\%) & $2168(85 \%)$ & $1825(83 \%)$ \\
\hline Present & $362(17 \%)$ & $378(19 \%)$ & $380(15 \%)$ & $385(17 \%)$ \\
\hline Missing & 0 & 0 & 0 & 0 \\
\hline \multicolumn{5}{|l|}{ Psychotic symptoms } \\
\hline Not present & 1941 (92\%) & 1930 (95\%) & 1950 (77\%) & $1936(88 \%)$ \\
\hline Present & $177(8 \%)$ & $97(5 \%)$ & $574(22 \%)$ & $274(12 \%)$ \\
\hline Missing & 0 & 0 & $24(1 \%)$ & 0 \\
\hline \multicolumn{5}{|l|}{ Disorders of anxiety/depression and psychotic symptoms } \\
\hline Neither & $1630(77 \%)$ & 1509 (74\%) & $1630(64 \%)$ & 1509 (69\%) \\
\hline $\begin{array}{l}\text { Disorders of anxiety/depression without psychotic } \\
\text { symptoms }\end{array}$ & $311(15 \%)$ & $421(21 \%)$ & $311(12 \%)$ & $421(19 \%)$ \\
\hline $\begin{array}{l}\text { Disorders of anxiety/depression with psychotic } \\
\text { symptoms }\end{array}$ & $177(8 \%)$ & $97(5 \%)$ & $177(7 \%)$ & $97(4 \%)$ \\
\hline Missing & & & 430 (17\%) & $183(8 \%)$ \\
\hline \multicolumn{5}{|l|}{ Negative symptoms } \\
\hline Not present & $1850(87 \%)$ & $1785(88 \%)$ & $2223(87 \%)$ & $1938(88 \%)$ \\
\hline Present & $268(13 \%)$ & $240(12 \%)$ & 325 (13\%) & $270(12 \%)$ \\
\hline Missing & 0 & $2(0.1 \%)$ & 0 & $2(0.1 \%)$ \\
\hline \multicolumn{5}{|l|}{ Suicidal thoughts } \\
\hline None & $1819(86 \%)$ & 1735 (86\%) & $2192(86 \%)$ & $1883(85 \%)$ \\
\hline At 1 time point & 245 (12\%) & $236(12 \%)$ & $293(12 \%)$ & $264(12 \%)$ \\
\hline At 2 time points & $46(2 \%)$ & $47(2 \%)$ & $54(2 \%)$ & $54(2 \%)$ \\
\hline At 3 time points & $8(0.4 \%)$ & $9(0.4 \%)$ & $9(0.4 \%)$ & $9(1 \%)$ \\
\hline \multicolumn{5}{|l|}{ General help seeking } \\
\hline No & 1923 (91\%) & 1717 (85\%) & 2314 (91\%) & $1870(85 \%)$ \\
\hline Yes & $195(9 \%)$ & $310(15 \%)$ & $233(9 \%)$ & 340 (15\%) \\
\hline Missing & 0 & 0 & $1(0.04 \%)$ & 0 \\
\hline \multicolumn{5}{|l|}{ Psychiatric medication use (nonpsychotic) } \\
\hline No & $2114(100 \%)$ & 2013 (99\%) & $2539(100 \%)$ & 2194 (99\%) \\
\hline Yes & $4(0.2 \%)$ & $14(1 \%)$ & $8(0.3 \%)$ & $16(1 \%)$ \\
\hline Missing & 0 & 0 & $1(0.1 \%)$ & 0 \\
\hline \multicolumn{5}{|l|}{ Any drug use $>5$ times } \\
\hline No & $1690(80 \%)$ & $1482(73 \%)$ & $1979(77 \%)$ & $1600(72 \%)$ \\
\hline Yes & $412(19 \%)$ & $526(26 \%)$ & $529(21 \%)$ & $589(27 \%)$ \\
\hline
\end{tabular}


Table 1 Continued

\begin{tabular}{|c|c|c|c|c|}
\hline & \multicolumn{2}{|c|}{ Risk Set } & \multicolumn{2}{|c|}{ Total Sample } \\
\hline & T2 & T3 & T2 & T3 \\
\hline Missing & $16(1 \%)$ & $19(1 \%)$ & $40(2 \%)$ & $21(1 \%)$ \\
\hline \multicolumn{5}{|l|}{ Cannabis use $>5$ times } \\
\hline No & $1679(79 \%)$ & $1474(73 \%)$ & 1966 (77\%) & $1592(72 \%)$ \\
\hline Yes & $404(19 \%)$ & $512(25 \%)$ & $519(25 \%)$ & $519(20 \%)$ \\
\hline Missing & $35(2 \%)$ & $41(2 \%)$ & $63(3 \%)$ & $44(2 \%)$ \\
\hline \multicolumn{5}{|l|}{ Trauma } \\
\hline No & $1715(81 \%)$ & $1641(81 \%)$ & $2052(81 \%)$ & $1783(81 \%)$ \\
\hline Yes & $403(19 \%)$ & $386(19 \%)$ & 496 (19\%) & 427 (19\%) \\
\hline \multicolumn{5}{|l|}{ Mean (SD) number of life events } \\
\hline Recent life events (T0) & $4.18(3.03)$ & $3.88(3.13)$ & $4.22(3.08)$ & $3.91(3.15)$ \\
\hline Recent life events $(\mathrm{TO}+1 \mathrm{y})$ & $5.28(3.38)$ & $5.01(3.58)$ & $5.38(3.43)$ & $5.06(3.62)$ \\
\hline Recent life events $(\mathrm{TO}+2 \mathrm{y})$ & $6.85(3.66)$ & $6.35(3.92)$ & $6.91(3.68)$ & $6.42(3.94)$ \\
\hline Recent life events $(\mathrm{TO}+3 \mathrm{y})$ & $6.84(3.73)$ & $6.34(3.96)$ & $6.90(3.73)$ & $6.41(3.98)$ \\
\hline \multicolumn{5}{|l|}{ Urbanicity } \\
\hline Urban & $1488(70 \%)$ & $1427(70 \%)$ & 1796 (71\%) & $1558(71 \%)$ \\
\hline Rural & $630(30 \%)$ & $600(30 \%)$ & 752 (29\%) & $652(29 \%)$ \\
\hline \multicolumn{5}{|c|}{ Proband's report of familial psychopathology } \\
\hline No & $1684(80 \%)$ & 1596 (79\%) & 2018 (79\%) & 1744 (79\%) \\
\hline Yes & 421 (20\%) & 421 (21\%) & $516(20 \%)$ & $453(20 \%)$ \\
\hline Missing & $13(0.6 \%)$ & $10(0.5 \%)$ & $14(1 \%)$ & $13(1 \%)$ \\
\hline
\end{tabular}




\section{DISCUSSION}

\section{Findings}

Psychotic symptoms were reported in $27 \%$ of individuals with a disorder of anxiety/ depression in a large sample of adolescents and young adults from the general population. Individuals with a disorder of anxiety/depression were also more likely to report psychotic symptoms than individuals without such a disorder. Both anxiety/depression disorder groups, ie, with and without psychotic symptoms, differed from controls (indicating lack of qualitative differences), but anxiety/depression with psychotic symptoms showed larger effect sizes than anxiety/depression without psychotic symptoms (indicating quantitative differences). Thus anxiety/depression disorders with and without psychotic symptoms were distinguished quantitatively by indicators of severity, course, onset, and environmental and familial risk. The findings could not be attributed to comorbidity of anxiety and depression per se because the sensitivity analyses showed that the pattern of results was largely robust to exclusion of this comorbidity.

\section{Disorders of Anxiety/Depression With Psychosis: A Prevalent and More Severe Disorder}

The present findings confirm earlier work that psychopathological dimensions of affective dysregulation and reality distortion often co-occur ${ }^{3,5,14,17}$. Most research on this co-occurrence was published from the perspective of psychosis research, thus focusing on additional presence of disorders of anxiety/depression in individuals endorsing psychotic phenomena. However, the present study joins a novel line of research that takes an alternative perspective. Although previous work has shown that psychotic symptoms are common in clinical samples presenting with disorders of anxiety/depression ${ }^{7,8}$, the current study aimed to quantify (1) the prevalence and (2) the consequences of psychotic symptoms in individuals with disorders of anxiety/depression in an epidemiological general population sample. 


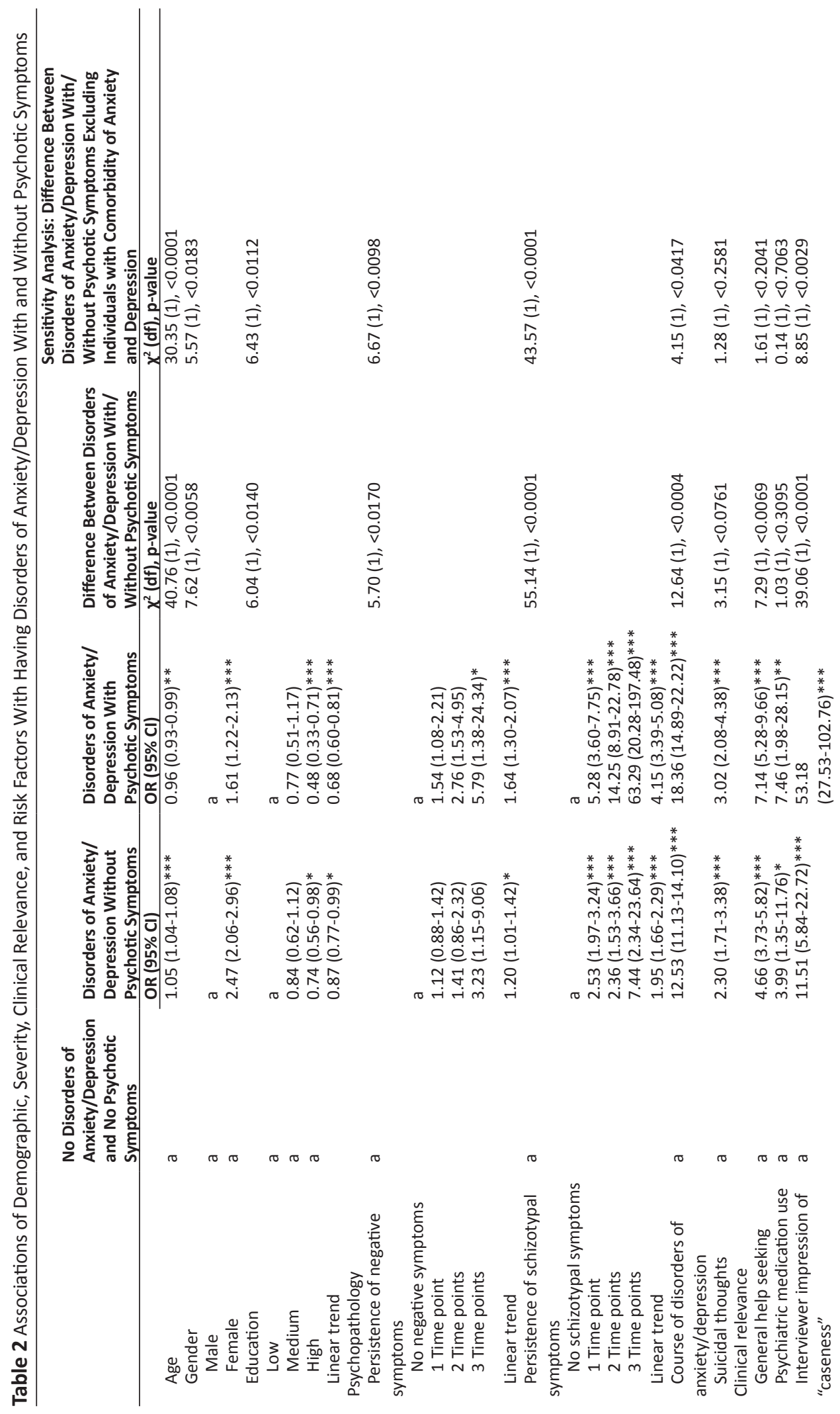




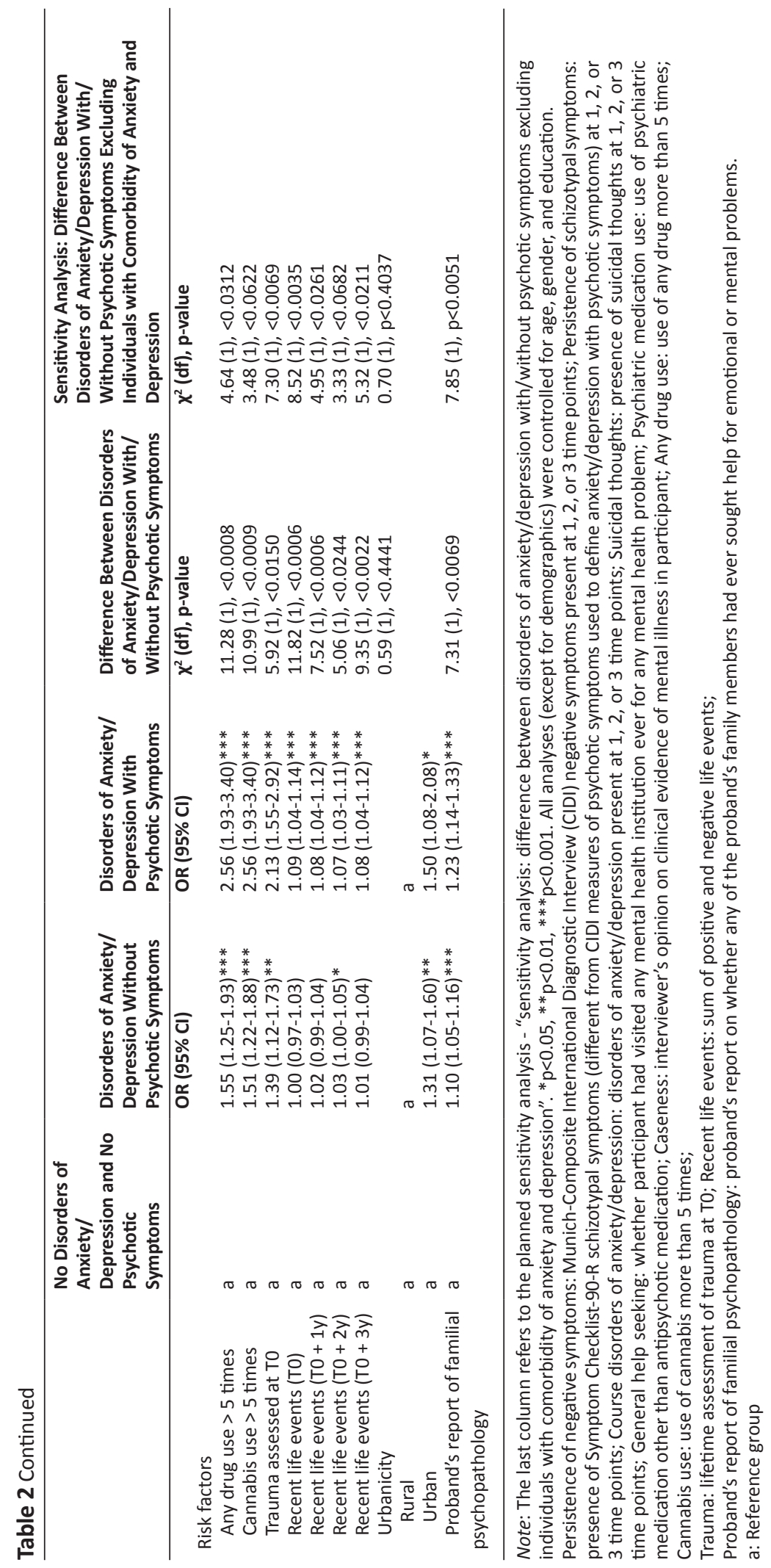

166 | Surviving Childhood 
The findings indicate that the sizeable subgroup of individuals with disorders of anxiety/ depression and psychotic symptoms has a more severe condition than those without psychotic symptoms. These findings are in agreement with studies showing that comorbidity of affective and psychotic psychopathology is more severe and of poorer prognosis than disorders without multidimensional admixture ${ }^{41}$. It also validates the interpretation of psychotic symptoms as complicating factors in disorders of anxiety/depression, characterized by a group of individuals with more severe pathology, earlier need for care, and more etiological loading.

The results support a hypothesized continuum of vulnerability between affective dysregulation and reality distortion, in which individuals who are vulnerable for either dimension are also more prone to develop the other ${ }^{7,12,42}$. Our results support this notion by showing that individuals with disorders of anxiety/depression are more prone to develop psychotic symptoms.

\section{Disorders of Anxiety/Depression, Psychotic Symptoms, and UHR Status}

Another explanation relevant to these findings is that individuals with disorders of anxiety/ depression and psychotic symptoms are in a prodromal stage of psychotic disorder. The rationale for this reasoning is that affective symptoms (1) form an intrinsic dimension of psychosis ${ }^{29}$, are the most common (and earliest) retrospectively reported prodromal symptoms ${ }^{6}$ that may come online earlier than psychotic phenomena that form the core characteristics of UHR status in help-seeking individuals, and (3) predict transition and worse outcome in UHR samples ${ }^{11,43}$. This interpretation, however, is not likely, given the (1) very large number of individuals in the present study reporting both disorders of anxiety/depression and psychotic symptoms, (2) the low prevalence of psychotic disorder in the population, and (3) the low number of individuals within the group at ultra-high risk for psychosis that actually make the transition to psychotic disorder ${ }^{43}$. Conversely, however, it can be considered likely that many of the help-seeking individuals presenting as UHR for psychosis actually represent the group with disorders of anxiety/depression with comorbid psychotic symptoms. In other words, a substantial proportion of helpseeking individuals presenting as UHR may in fact present with disorders of anxiety/ depression complicated by psychotic symptoms, which is supported by (1) the observation that the great majority of UHR individuals initially carries a diagnosis of anxiety disorder and/or depression ${ }^{9-11}$ and (2) reports that part of this group responds favourable to antidepressant medication ${ }^{44}$. Because disorders of anxiety/depression have traditionally been considered as "nonpsychotic", this explanation has not been considered before.

\section{Diagnostic Implications}

Mental disorders nosology is based on the theory that the signs and symptoms associated with mental ill health fluctuate as a function of an underlying latent diagnostic construct. However, disorders in practice do not occur in isolation, be it a combination of affective 
and psychotic disorders ${ }^{3}$ or mental disorder diagnoses in genera ${ }^{45}$. The data, in combination with previous work, suggest that psychopathology may be considered as a network of symptom dimensions that reciprocally impact on each other over time and are linked as part of a homeostatic mechanism, time, and/or shared liability. An approach that possibly can accommodate this type of variation over time is the clinical staging model ${ }^{46}$, in combination with a network model of psychopathology ${ }^{47}$. Thus, reciprocally impacting symptoms sharing degrees of liability may develop across stages of severity and comorbidity.

\section{Methodological Issues}

The current results should be interpreted in light of the strengths and limitations of the study. A major strength of the study was that it addressed the copresence of disorders of anxiety/depression and psychotic symptoms, assessed by psychologists who were allowed to probe with clinical questioning, in a large, representative population study that was followed over an extended period. However, the relatively long follow-up period with only 3 assessments also limits the possibilities of constructing dynamics, microlevel models of the development of reciprocally impacting domains of psychopathology. Second, even though psychotic symptoms were assessed by psychologists using clinical questioning, false-positive ratings are likely to have occurred. However, the effect of this would be conservative rather that to give rise to spurious associations. Furthermore, even "falsepositive" ratings of positive psychotic symptoms have been shown to be predictive of later (psychotic and affective) pathology ${ }^{25}$ and therefore are important predictors to include. Third, some of the risk factors that were investigated should be interpreted carefully. For example, the variable reflecting caseness was assigned by the same interviewer enquiring about psychopathology and therefore may be biased. Finally, some of the assessments of the exposure variables may have been biased because individuals who are more severely ill may report greater degree of exposure to, eg, childhood trauma or life events. 


\section{Funding}

European Union (EU) Interreg IVb project "Health and Demographic Changes" (30972507N): European Community's Seventh Framework Program (HEALTH-F2-2009-241909, Project EU-GEI); German Federal Ministry of Education and Research (BMBF) (01EB9405/6, 01EB9910/6, EB10106200, 01EB0140, 01EB0440); Deutsche Forschungsgemeinschaft (LA1148/1-1, WI2246/7-1, WI709/8-1).

\section{Acknowledgements}

This work is part of the EDSP study. Core staff members of the EDSP group are Dr. Kirsten von Sydow, Dr Gabriele Lachner, Dr Axel Perkonigg, Dr Peter Schuster, Dipl.-Stat. Michael Höfler, Dipl.-Psych. Holger Sonntag, Dipl.-Psych. Tanja Brückl, Dipl.-Psych. Elzbieta Garczynski, Dr Barbara Isensee, Dipl.-Psych. Agnes Nocon, Dr Chris Nelson, Dipl.-Inf. Hildegard Pfister, Dr Victoria Reed, Dipl.-Soz. Barbara Spiegel, Dipl.-Psych. Andrea Schreier, Dr Ursula Wunderlich, Dr Petra Zimmermann, Dipl.-Psych. Datja Beesdo, and Dipl.-Psych. Antje Bitner. Scientific advisors are Dr Jules Angst (Zurich), Dr. Jürgen Margraf (Basel), Dr Günther Esser (Potsdam), Dr Kathleen Merikangas (National Institute of Mental Health [NIMH], Bethesda), Dr Ron Kessler (Harvard, Boston), and Dr Jim van Os (Maastricht). 


\section{REFERENCES}

1. Kotov R, Chang SW, Fochtmann LJ, Mojtabai R, Carlson GA, Sedler MJ, Bromet EJ. Schizophrenia in the internalizing-externalizing framework: a third dimension? Schizophr Bull Nov 2011;37(6):1168-1178.

2. Krabbendam L, Myin-Germeys I, de Graaf R, Vollebergh W, Nolen WA, ledam J, van Os J. Dimensions of depression, mania and psychosis in the general population. Psychological Medicine 2004;34:1177-1186.

3. Buckley PF, Miller BJ, Lehrer DS, Castle DJ. Psychiatric comorbidities and schizophrenia. Schizophr Bull Mar 2009;35(2):383-402.

4. Huppert JD, Smith TE. Anxiety and schizophrenia: the interaction of subtypes of anxiety and psychotic symptoms. CNS spectrums

Sep 2005;10(9):721-731.

5. Boks MP, Leask S, Vermunt JK, Kahn RS. The structure of psychosis revisited: the role of mood symptoms.

Schizophr Res Jul 2007;93(1-3):178-185.

6. Hafner H, Maurer K, Trendler G, an der Heiden W, Schmidt M. The early course of schizophrenia and depression*. Eur Arch Psychiatry Clin Neurosci Jun 2005;255(3):167-173.

7. Hanssen $M$, Peeters $F$, Krabbendam $L$, Radstake $\mathrm{S}$, Verdoux H, van Os J. How psychotic are individuals with non-psychotic disorders? Soc Psychiatry Psychiatr Epidemiol Mar 2003;38(3):149-154.

8. Olfson $M$, Lewis-Fernandez $R$, Weissman MM, Feder A, Gameroff MJ, Pilowsky D, Fuentes M. Psychotic symptoms in an urban general medicine practice. Am J Psychiatry Aug 2002;159(8):1412-1419.
9. Addington J, Cornblatt BA, Cadenhead $\mathrm{KS}$, et al. At clinical high risk for psychosis: outcome for nonconverters. Am J Psychiatry Aug 2011;168(8):800-805.

10. Velthorst E, Nieman DH, Becker HE, et al. Baseline differences in clinical symptomatology between ultra high risk subjects with and without a transition to psychosis. Schizophr Res Apr 2009;109(1-3):60-65.

11. Yung AR, Phillips LJ, Yuen HP, McGorry PD. Risk factors for psychosis in an ultra high-risk group: psychopathology and clinical features. Schizophrenia Research 2004;67:131-142.

12. Verdoux $H$, van Os J, Maurice-Tison $S$, Gay B, Salamon R, Bourgeois ML. Increased occurrence of depression in psychosis-prone subjects: a follow-up study in primary care settings. Compr Psychiatry Nov-Dec 1999;40(6):462-468.

13. Armando $M$, Nelson $B$, Yung $A R$, Ross M, Birchwood M, Girardi P, Fiori Nastro P. Psychotic-like experiences and correlation with distress and depressive symptoms in a community sample of adolescents and young adults. Schizophr Res Jun 2010;119(1-3):258-265.

14. Varghese D, Scott J, Welham J, Bor W, Najman J, O'Callaghan M, Williams G, McGrath J. Psychotic-like experiences in major depression and anxiety disorders: a population-based survey in young adults. Schizophr Bull Mar 2011;37(2):389-393.

15. Garety PA, Kuipers E, Fowler D, Freeman D, Bebbington PE. A cognitive model of the positive symptoms of psychosis. Psychol Med

Feb 2001;31(2):189-195. 
16. Freeman D, Garety PA. Connecting neurosis and psychosis: the direct influence of emotion on delusions and hallucinations. Behav Res Ther Aug 2003;41(8):923-947.

17. Van Rossum I, Dominguez MD, Lieb R, Wittchen HU, Van Os J. Affective dysregulation and reality distortion: a 10-year prospective study of their association and clinical relevance.

Schizophrenia Bulletin 2011;37(3):561-571.

18. Lichtenstein P, Yip BH, Björk C, Pawitan Y, Cannon TD, Sullivan PF, Hultman CM. Common genetic determinants of schizophrenia and bipolar disorder in Swedish families: a population-based study. Lancet 2009;373:234-239.

19. Weiser M, van Os J, Davidson M. Time for a shift in focus in schizophrenia: from narrow phenotypes to broad endophenotypes. Br J Psychiatry Sep 2005;187:203-205.

20. Mortensen PB, Pedersen MG, Pedersen CB. Psychiatric family history and schizophrenia risk in Denmark: which mental disorders are relevant? Psychol Med Feb 2010;40(2):201-210.

21. Breetvelt EJ, Boks MP, Numans ME, Selten JP, Sommer IE, Grobbee DE, Kahn RS, Geerlings MI. Schizophrenia risk factors constitute general risk factors for psychiatric symptoms in the population. Schizophr Res Jul 2010;120(1-3):184-190.

22. Bora $E$, Yucel $M$, Pantelis $C$. Cognitive impairment in schizophrenia and affective psychoses: implications for DSM-V criteria and beyond. Schizophr Bull Jan 2010;36(1):36-42.
23. Chapman LJ, Chapman JP, Kwapil TR, Eckblad M, Zinser MC. Putatively psychosis-prone subjects 10 years later. J Abnorm Psychol May 1994;103(2):171-183.

24. Rossler W, Hengartner MP, AjdacicGross V, Haker H, Gamma A, Angst J. Sub-clinical psychosis symptoms in young adults are risk factors for subsequent common mental disorders. Schizophr Res Sep 2011;131(1-3):18-23.

25. van Nierop M, Van Os J, Gunther N, et al. Phenotypically continuous with clinical psychosis, discontinuous in need for care: Evidence for an extended psychosis phenotype. Schizophrenia Bull 2012;38(2):231-238.

26. Kaymaz N, Drukker M, Lieb R, Wittchen $H$, Werbeloff $N$, Weiser M, Lataster T, Van Os J. Do subthreshold psychotic experiences predict clinical outcomes in unselected non-helpseeking population-based samples? A systematic review and meta-analysis, enriched with new results. Psychol Med 2012;DOI:10.1017/ S0033291711002911.

27. Krabbendam L, Myin-Germeys I, Hanssen M, De Graaf R, Vollebergh W, Bak M, van Os J. Development of depressed mood predicts onset of psychotic disorder in individuals who report hallucinatory experiences. British Journal of Clinical Psychology 2005;44:113-125. 
28. Perlis $\mathrm{RH}$, Uher $\mathrm{R}$, Ostacher $\mathrm{M}$, Goldberg JF, Trivedi MH, Rush AJ, Fava M. Association between bipolar spectrum features and treatment outcomes in outpatients with major depressive disorder. Arch Gen Psychiatry Apr 2011;68(4):351-360.

29. van Os J, Kenis G, Rutten BPF. The environment and schizophrenia. Nature 2010;468(7321):203-212.

30. Lieb R, Isensee B, von Sydow K, Wittchen HU. The Early Developmental Stages of Psychopathology Study (EDSP): a methodological update. European addiction research Dec 2000;6(4):170-182.

31. Wittchen HU, Perkonigg A, Lachner G, Nelson CB. Early developmental stages of psychopathology study (EDSP): objectives and design.

European addiction research Mar 1998;4(1-2):18-27.

32. Beesdo K, Pine DS, Lieb R, Wittchen $\mathrm{HU}$. Incidence and risk patterns of anxiety and depressive disorders and categorization of generalized anxiety disorder. Arch Gen Psychiatry Jan 2010;67(1):47-57.

33. Wittchen $\mathrm{H}$, Pfister H. DIA-XInterviews: Manual fur ScreeningVerfahren und Interview; Interviewheft Langsschnittuntersuchung (DIA-XLifetime); Enganzungsheft (DIA-XLifetime); Interviewheft Querschnittsuntersuchung (DIA-XMonatsversion); Erganzungsheft (DIA-X-12 Monatsversion); PC-Programm zur Durchfuhrung der Interviews (Langsund Querschnittsuntersuchung). Ausertungsprogramm. Frankfurt, Germany: Swets \& Zeiltinger; 1997.
34. Dominguez MD, Saka MC, Lieb R, Wittchen $\mathrm{H}$, van Os J. Early expression of negative/disorganized symptoms predicting psychotic experiences and subsequent clinical psychosis: a 10-year study. American Journal of Psychiatry 2010;167(9):1075-1082.

35. Smeets $F$, Lataster T, Dominguez MG, Hommes J, Lieb R, Wittchen HU, van Os J. Evidence That Onset of Psychosis in the Population Reflects Early Hallucinatory Experiences That Through Environmental Risks and Affective Dysregulation Become Complicated by Delusions. Schizophrenia Bulletin 2012;38(3):531-542.

36. Dominguez MD, Wichers $M$, Lieb $R$, Wittchen HU, van Os J. Evidence that onset of clinical psychosis is an outcome of progressively more persistent subclinical psychotic experiences: an 8-year cohort study. Schizophr Bull Jan 2011;37(1):84-93.

37. Henquet $C$, Krabbendam L, Spauwen J, Kaplan C, Lieb R, Wittchen HU, van Os J. Prospective cohort study of cannabis use, predisposition for psychosis, and psychotic symptoms in young people. BMJ (Clinical research ed) Jan 1 2005;330(7481):11.

38. Spauwen J, Krabbendam L, Lieb R, Wittchen HU, van Os J. Impact of psychological trauma on the development of psychotic symptoms: relationship with psychosis proneness. Br J Psychiatry 2006;188:527-533.

39. Spauwen J, Krabbendam L, Lieb R, Wittchen HU, van Os J. Does urbanicity shift the population expression of psychosis? J Psychiatr Res Nov-Dec 2004;38(6):613-618. 
40. Penninx BW, Nolen WA, Lamers F, et al. Two-year course of depressive and anxiety disorders: results from the Netherlands Study of Depression and Anxiety (NESDA). J Affect Disord Sep 2011;133(1-2):76-85.

41. Saarni SI, Viertio S, Perala J, Koskinen S, Lonnqvist J, Suvisaari J. Quality of life of people with schizophrenia, bipolar disorder and other psychotic disorders. Br J Psychiatry Nov 2010;197(5):386-394.

42. Lin A, Wood SJ, Nelson B, et al. Neurocognitive predictors of functional outcome two to 13 years after identification as ultra-high risk for psychosis. Schizophr Res Oct 2011;132(1):1-7.

43. Yung $A R$, Yuen $H P$, Berger $G$, Francey $S$, Hung TC, Nelson B, Phillips L, McGorry P. Declining transition rate in ultra high risk (prodromal) services: dilution or reduction of risk? Schizophr Bull May 2007;33(3):673-681.

44. Cornblatt B, Lencz T, Obuchowski M. The schizophrenia prodrome: treatment and high-risk perspectives.

Schizophr Res

Mar 1 2002;54(1-2):177-186.

45. Kessler RC, Ormel J, Petukhova M, et al. Development of lifetime comorbidity in the World Health Organization world mental health surveys.

Arch Gen Psychiatry Jan 2011;68(1):90-100.

46. McGorry PD, Nelson B, Goldstone S, Yung AR. Clinical Staging: A Heuristic and Practical Strategy for New Research and Better Health and Social Outcomes for Psychotic and Related Mood Disorders. Canadian Journal of Psychiatry 2010;55(8):486-497.
47. Borsboom D, Cramer AO, Schmittmann VD, Epskamp S, Waldorp LJ. The small world of psychopathology. PLoS One 2011;6(11):e27407. 


\title{
Childhood Trauma Is Associated With a Specific Admixture of Affective, Anxiety, and Psychosis Symptoms Cutting Across Traditional Diagnostic Boundaries
}

\author{
Martine van Nierop ${ }^{A}$ \\ Wolfgang Viechtbauer ${ }^{A}$ \\ Nicole Gunther ${ }^{B}$ \\ Catherine van Zelst ${ }^{\mathrm{A}}$ \\ Ron de Graafc \\ Margreet ten Have ${ }^{c}$ \\ Saskia van Dorsselaer ${ }^{\mathrm{C}}$ \\ Maarten Bak ${ }^{\mathbf{A}}$ \\ Genetic Risk and OUtcome of Psychosis (GROUP) investigators* \\ Ruud van Winkel ${ }^{A D}$ \\ * The GROUP investigators are: \\ Richard Bruggeman ${ }^{\mathrm{E}}$ \\ Wiepke Cahn ${ }^{\mathrm{F}}$ \\ Lieuwe de Haan ${ }^{G}$ \\ Rene S. Kahn ${ }^{\mathrm{F}}$ \\ Carin Meijer ${ }^{\mathrm{G}}$ \\ Inez Myin-Germeys ${ }^{\mathrm{A}}$ \\ Jim van $\mathrm{Os}^{\mathrm{A}, \mathrm{H}}$ \\ Durk WiersmaE
}

\footnotetext{
A Dept. of Psychiatry and Psychology, School for Mental Health and Neuroscience, EURON, Maastricht University Medical Centre, PO Box 616 (Vijv1), 6200 MD Maastricht, The Netherlands

B Faculty of Psychology and Educational Sciences, Open University, The Netherlands

C Netherlands Institute of Mental Health and Addiction, Da Costakade 45, 3521 VS Utrecht, The Netherlands

D University Psychiatric Center Katholieke Universiteit Leuven, campus Kortenberg, Leuvensesteenweg, Kortenberg, Belgium

E University Medical Center Groningen, Department of Psychiatry, Groningen, The Netherlands

F University Medical Center Utrecht, Department of Psychiatry, Rudolf Magnus Institute of Neuroscience, Utrecht,

The Netherlands

GAcademic Medical Centre, University of Amsterdam, Department of Psychiatry, Amsterdam, The Netherlands

H King's College London, King's Health Partners, Department of Psychosis Studies, Institute of Psychiatry, London, United Kingdom
}

Psychological Medicine 2014, doi: 10.1017/S0033291714002372 


\section{ABSTRACT}

\section{Background}

Meta-analyses link childhood trauma to depression, mania, anxiety disorders, and psychosis. It is unclear, however, whether these outcomes truly represent distinct disorders following childhood trauma, or that childhood trauma is associated with admixtures of affective, psychotic, anxiety and manic psychopathology throughout life.

\section{Methods}

We used data from a representative general population sample (NEMESIS-2; $n=6646$ ), of whom respectively 1577 and 1120 had a lifetime diagnosis of mood or anxiety disorder, as well as from a sample of patients with a diagnosis of schizophrenia (GROUP; $n=825$ ). Multinomial logistic regression was used to assess whether childhood trauma was more strongly associated with isolated affective/psychotic/anxiety/manic symptoms than with their admixture.

\section{Results}

In NEMESIS-2, largely comparable associations were found between childhood trauma and depression, mania, anxiety and psychosis. However, childhood trauma was considerably more strongly associated with their lifetime admixture. These results were confirmed in the patient samples, in which it was consistently found that patients with a history of childhood trauma were more likely to have a combination of multiple symptom domains compared to their non-traumatized counterparts. This pattern was also found in exposed individuals who did not meet criteria for a psychotic, affective or anxiety disorder and who did not seek help for subclinical psychopathology.

\section{Conclusion}

Childhood trauma increases the likelihood of a specific admixture of affective, anxiety and psychotic symptoms cutting across traditional diagnostic boundaries, and this admixture may already be present in the earliest stages of psychopathology. These findings may have significant etiological, pathophysiological, diagnostic and clinical repercussions. 


\section{INTRODUCTION}

Childhood trauma has been robustly associated with a number of mental disorders, including schizophrenia ${ }^{1}$, depression ${ }^{2}$, anxiety ${ }^{3}$ and bipolar disorder ${ }^{4}$. In a recent meta-analysis on the link between childhood trauma and schizophrenia in comparison to other disorders, Matheson and colleagues found no clear evidence that childhood trauma showed a stronger association with schizophrenia than with depression or bipolar disorder ${ }^{5}$. The authors concluded that, instead of increasing risk for a specific disorder, childhood trauma may increase risk for stress-related disorders through changes of the hypothalamic-pituitary-adrenal axis, alterations of which have been reported in a number of mental disorders including psychosis $^{6}$, depression ${ }^{7}$, anxiety ${ }^{8}$ and mania ${ }^{9}$.

While it is theoretically plausible that the assumed causal effect of childhood trauma would induce comparable biological alterations in different individuals, the issue why some individuals go on to develop depression, while others develop psychosis or mania, remains unresolved. A popular explanation is that one's individual genetic background may determine the expression of psychopathology after exposure to a harmful environmental factor ${ }^{10}$. However, for childhood trauma, these models have not always been supported by the data at hand. For example, a number of studies found no evidence for an interaction between familial risk for psychosis and exposure to childhood trauma ${ }^{11-13}$.

It has also been observed that the early stages of psychopathology are characterized by a mixed collection of symptoms not specific for any one mental disorder ${ }^{14}$. Studies on highrisk individuals have also shown that predicting which individual is going to develop a particular disorder is extremely difficult ${ }^{15}$.

Taken together, these findings seem difficult to reconcile with the relatively simplistic notion that a person at above-average genetic risk for depression may develop an affective disorder following childhood trauma, whereas a person at higher genetic risk for schizophrenia would develop a psychotic outcome.

An alternative explanation is that childhood trauma may not specifically induce a particular disorder, but rather the co-occurrence of depression, psychosis, mania and anxiety over the life course. Depending on which symptoms prevail at any given point, diagnoses given to individual patients may then vary. Some research is available that reports associations between childhood trauma and clustering of multiple symptom domains, such as psychosis combined with depression ${ }^{16}$. If indeed childhood trauma is associated with the admixture of different symptom domains rather than specific mental disorders in individual patients, this would have important repercussions for the treatment of traumatized individuals presenting with mental symptoms ${ }^{17}$, as well as for etiological models involving traumainduced mental disorders ${ }^{18}$.

However, if evidence for trauma-related admixture would be found, one could argue that a stronger association with the admixture of symptom domains may simply reflect 
increased severity in these individuals, as for example, when depressive symptoms become more severe, subjects may be more likely to also experience psychotic symptoms. A related hypothesis is that childhood trauma may initially give rise to affective symptoms, and only later to psychotic symptoms, which has been referred to as "the affective pathway to psychosis ${ }^{19}$.

Therefore, the present study investigates whether childhood trauma is associated with specific isolated mental symptoms separately, or with the admixture of affective, anxious as well as psychotic symptoms in a representative, general population sample, with additional analyses investigating alternative hypotheses (increased severity of psychopathology and the affective pathway to psychosis). Moreover, the possible admixture of symptom domains following childhood trauma may have limited clinical relevance if it is only found in general population samples but not in clinical samples. Therefore, similar analyses were performed in a sample of patients with a lifetime mood disorder, a sample of patients with a lifetime anxiety disorder, and in a sample with a (nonaffective) psychotic disorder.

\section{METHODS}

\section{General population sample}

\section{NEMESIS-2}

The representative, general population sample forms part of the second Netherlands Mental Health Survey and Incidence Study (NEMESIS-2), a longitudinal study of mental disorders in the Dutch general population. For the present study, the baseline and three year follow-up measurements were used. The study was approved by the Medical Ethics Review Committee for Institutions on Mental Health Care. Participants aged 18-65 years were included; insufficient fluency in Dutch was an exclusion criterion. Participants were interviewed by trained interviewers, who were not clinicians, with the Composite International Diagnostic Interview (CIDI) version 3.0 ${ }^{20}$, and additional questionnaires.

A more detailed description of NEMESIS- 2 methodology was presented by De Graaf and colleagues ${ }^{21}$.

\section{Assessment of environmental factors}

Childhood trauma was assessed using a format based on the NEMESIS-1 trauma questionnaire ${ }^{21}$. Whenever a subject reported having experienced one of five types of childhood trauma (emotional neglect, physical abuse, psychological abuse, sexual abuse and peer victimization; before the age of 16), they were asked to state how often it had occurred, on a scale with possible scores of 1 (once), 2 (sometimes), 3 (regularly), 4 (often), 
and 5 (very often). Factor analysis indicated that the five childhood trauma scales loaded onto one factor (eigenvalue 2.47); therefore one childhood trauma scale (range 0-25) was constructed consisting of the sum of the five childhood trauma types, in agreement with previous studies ${ }^{22}$. Cannabis use has been implicated in the development of various mental disorders ${ }^{23}$ and may also be associated with childhood trauma ${ }^{24}$. Therefore, analyses were repeated using cannabis use as a possible confounder. Cannabis use was assessed in the section Illegal Substance Use of the CIDI 3.0. Subjects were asked to state whether they had ever (baseline) or in the previous three years (follow-up) used cannabis, and, if so, rated frequency of use in the period of most frequent use on a scale of 1 (less than once per month) to 6 (every day).

\section{Symptom domains}

Depressive, manic and anxiety symptoms were assessed with the CIDI 3.020. For depression, participants were asked if they ever had (at baseline) or in the time between baseline and follow-up experienced an episode lasting a number of consecutive days during which they felt low, depressed, or had a lack of interest. If endorsed, subjects were asked if, during this period, they had experienced any of a list of 28 depressive symptoms (see Appendix 1).

For mania, subjects were asked whether they had experienced a period of several days of feeling increased excitement, energy or irritability at baseline (lifetime) or follow-up (incidence). If endorsed, they were asked if they experienced any of a list of 18 symptoms (see Appendix 2).

Similarly, for anxiety, subjects were asked whether they had experienced a period in which they felt sudden attacks of anxiety or panic (section Panic Disorder [PD]), increased worry or nervousness (section Generalized Anxiety Disorder [GAD]), or anxiety in social situations (section Social Phobia [SP]), at baseline or follow-up. If endorsed, they were asked whether they had experienced any of a list of 16 (section PD), 12 (section GAD) or 15 (section SP) symptoms. All items were added to one anxiety scale (43 symptoms, see Appendix 3 ).

Lastly, presence of psychotic experiences was assessed using a questionnaire specifically developed, based on the CIDI 1.1, for the purpose of assessing psychotic symptoms, since studies on earlier CIDI versions concluded that the instrument did not adequately measure psychotic symptoms ${ }^{25}$. Participants were asked at baseline or follow-up whether they had experienced any of a list of 20 positive psychotic symptoms (see Appendix 4). As clinical relevance of psychotic experiences may be difficult to diagnose by lay interviewers ${ }^{26}$, individuals who endorsed at least one psychotic experience were contacted for re-interview over the telephone by an experienced clinician within eight weeks after the initial interview, similar to the procedure in NEMESIS- $1^{27}$. Re-interviews were conducted using questions from the Structured Clinical Interview for DSM-IV (SCID-I), an instrument with proven reliability and validity in diagnosing psychotic disorders ${ }^{28}$. Findings from all re-interviews were discussed with a second clinician (MB). For the present study, only the validated psychotic experiences, as established by re-interview, were used. 
For each symptom category, symptoms were considered present when participants endorsed at least one symptom. All symptoms were assessed using 'yes' or 'no' questions, and for each symptom cluster sum scores were obtained by adding reported symptoms. If symptoms were endorsed, subjects were asked whether they had sought help for these symptoms.

\section{Sample and attrition}

A total of 6646 subjects participated at the baseline measurement, 5303 subjects participated at the three-year follow-up measurement (Table 1). Baseline symptom scores did not differ between participants who were available for follow-up and participants who were not, except for psychosis; subjects lost to follow-up reported fewer psychosis symptoms at baseline (mean 0.07) than subjects available for follow-up (mean 0.12, $t=-2.7$, $p=0.006$ ). Subjects who did not participate in the follow-up measurement were slightly younger at baseline than subjects who were available for follow-up (mean no follow-up 42.88 , mean $\left._{\text {follow-up }} 44.59, \mathrm{t}=-4.5, \mathrm{p}<0.001\right)$. There were no differences in sex as a function of attrition.

\section{Patient samples}

From the NEMESIS-2 sample, two clinical samples were derived: a sample of participants with a mood disorder (major depressive disorder, bipolar disorder or dysthymia, $n=1577$ ) and a sample of participants with an anxiety disorder (social phobia, panic disorder or generalized anxiety disorder, $n=1120$ ). Diagnoses were established using the CIDI 3.0. Since the number of patients with a non-affective psychotic disorder in NEMESIS- 2 was too small $(n=43)$, we used data from an independent sample of patients with a psychotic disorder, the Genetic Risk and Outcome of Psychosis (GROUP) sample ${ }^{29}$. In the GROUP study, patients with a diagnosis of non-affective psychotic disorder were recruited from five university hospitals in the Netherlands and Belgium (Groningen, Amsterdam, Maastricht, Utrecht, Louvain) and their affiliated mental health care institutions. Patients were eligible for inclusion if they: (1) were aged 16-65 years, (2) met the Diagnostic and Statistical Manual of Mental Disorders, Fourth Edition (DSM-IV) criteria for a non-affective psychotic disorder (in Amsterdam, Maastricht, Utrecht and Louvain assessed with the Comprehensive Assessment of Symptoms and History [CASH ${ }^{30}$; in Groningen with the Schedules for Clinical Assessment in Neuropsychiatry [SCAN 2.131]), (3) had first contact with mental health care less than ten years before initial assessment, and (4) were proficient in Dutch. Participants were excluded if their estimated level of intelligence was below 70 .

Participants who were assessed at the Amsterdam, Maastricht, Utrecht and Louvain sites were used for the present study as we used symptom data from the CASH, which was not assessed at the Groningen site. Childhood trauma in the GROUP study was assessed with the Dutch version of the Childhood Trauma Questionnaire (CTQ-SF ${ }^{32}$ ), a 25 item self-report 
questionnaire rated on a scale of 1 (never true) to 5 (very often true). The CTQ measures physical abuse, physical neglect, sexual abuse, emotional abuse and emotional neglect. A total trauma score was obtained by calculating the average of all 25 items. Symptom assessments in GROUP at baseline and follow-up included present state depression, anxiety and psychosis (positive psychotic symptoms) using the Positive and Negative Syndrome Scale (PANSS ${ }^{33}$ ). Depression and anxiety were assessed with one item each (depression: have you felt sad, down or depressed; anxiety: worried, nervous, restless or panicked), on a scale of 1 (absent) to 7 (extreme), indicating severity of the symptom. Present state mania was assessed at baseline and follow-up with the CASH, with one item (have you felt overly or abnormally exited or active) on a scale of 0 (no) to 5 (severe). Cannabis use was assessed with the CIDI 2.1 ${ }^{25}$. Subjects were asked to state whether they had ever (baseline) or in the previous three years (follow-up) used cannabis, and, if so, were rated on frequency of use in the period of most use on a scale of 1 (less than weekly) to 3 (daily).

\section{Sample}

In GROUP, 825 subjects participated at the baseline measurement, and 582 subjects were interviewed at the three-year follow-up measurement. Subjects lost to follow-up did not differ from subjects who were available for follow-up in terms of age, sex, anxiety, or mania symptom severity at baseline. Subjects lost to follow-up did, however, report a higher level of depressive symptoms (mean no follow-up $^{2.65}$, mean follow-up $2.36, t=2.57, p=0.011$ ) and psychosis symptoms ( mean $_{\text {no follow-up }} 1.97$, $^{\text {mean }}$ follow-up $_{1.80} \mathrm{t}=2.80, \mathrm{p}=0.005$ ) at baseline. 
Table 1 Baseline and first follow-up of NEMESIS-2 and GROUP - Demographics and symptom profiles

\begin{tabular}{|c|c|c|}
\hline & Baseline & Three-year follow-up \\
\hline NEMESIS-2 & $(\mathrm{N}=6,646)$ & $(\mathrm{N}=5,303)$ \\
\hline Sex (male), N (\%) & $2,976(45)$ & $2,381(45)$ \\
\hline No mood, anxiety or psychotic disorder, N (\%) & $3,986(63)$ & $3,913(61)$ \\
\hline Age, years & $44.3(12.5)$ & $47.6(12.4)$ \\
\hline Childhood trauma ${ }^{\mathrm{A}}$ & $1.7,0-25(3.2)$ & n.a. \\
\hline Depression symptom scale ${ }^{B}$ & $4.0,0-28(7.3)$ & $1.50-28(5.0)$ \\
\hline Mania symptom scale ${ }^{B}$ & $0.6,0-17(1.8)$ & $0.4,0-17(1.3)$ \\
\hline Anxiety symptom scale ${ }^{B}$ & $4.9,0-34(6.7)$ & $1.5,0-34(3.8)$ \\
\hline Psychosis symptom scale ${ }^{c}$ & $0.1,0-17(0.6)$ & $0.1,0-17(0.4)$ \\
\hline Cannabis use $\mathrm{D}^{\mathrm{D}}$ & $0.4,0-6(1.1)$ & $0.03,0-6(0.3)$ \\
\hline Education level ${ }^{\mathrm{E}}$ & $3.0,1-4(0.9)$ & $3.0,1-4(0.9)$ \\
\hline GROUP & $(\mathrm{N}=825)$ & $(\mathrm{N}=582)$ \\
\hline Sex (male), N (\%) & $634(77)$ & $446(77)$ \\
\hline Age, years & $27.4,16-61(7.9)$ & $30.7,18-60(7.4)$ \\
\hline Childhood trauma ${ }^{\mathrm{F}}$ & $1.6,1-3.8(0.5)$ & n.a. \\
\hline Depression symptom scale ${ }^{G}$ & $2.4,1-7(1.5)$ & $1.9,1-7(1.2)$ \\
\hline Mania symptom scale ${ }^{H}$ & $0.1,0-4(0.5)$ & $0.1,0-4(0.5)$ \\
\hline Anxiety symptom scale' & $2.3,1-6(1.3)$ & $2.0,1-6(1.2)$ \\
\hline Psychosis symptom scale & $1.8,1-5.3(0.8)$ & $1.6,1-4.1(0.7)$ \\
\hline Cannabis use $\mathrm{K}^{\mathrm{k}}$ & $1.7,0-3(1.4)$ & $0.7,0-3(1.2)$ \\
\hline Education level ${ }^{\mathrm{L}}$ & $4.1,0-8(2.1)$ & $4.4,0-8(2.1)$ \\
\hline
\end{tabular}

Note: NEMESIS-2, The second Netherlands Mental Health Survey and Incidence Study; GROUP: Genetic Risk and Outcome of Psychosis study; n.a., not applicable. Values given are mean, range (SD) unless otherwise stated

A Childhood trauma (NEMESIS-2) is a combination scale of 5 different types of trauma; emotional neglect (0 [never]-5[very often]), psychological abuse (0-5), physical abuse (0-5), sexual abuse (0-5) and peer victimization (0-5).

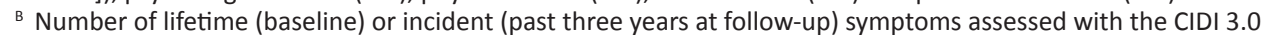

c Number of lifetime (baseline) or incident (past three year at follow-up) clinically validated positive psychotic symptoms assessed with a self-constructed questionnaire

D Cannabis use (NEMESIS-2) was rated as frequency of use in the period of most frequent use on a scale of 0 (never) to 6 (every day), lifetime (baseline) and incident (past three years, follow-up)

E Education level (NEMESIS-2); 1 (primary education), 2 (lower secondary education), 3 (higher secondary education), 4 (higher professional, university)

F Childhood trauma (GROUP) is an average score of 25 items (physical, sexual and emotional abuse, and physical and emotional neglect) each ranging 1 (never true) to 5 (very often true)

${ }^{G}$ Severity of present state (past two weeks) depression assessed with the PANSS

${ }^{H}$ Severity of present state (past month) mania assessed with the CASH

I Severity of present state (past two weeks) anxiety assessed with the PANSS

S Severity of present state (past two weeks) psychosis assessed with the PANSS

${ }^{k}$ Cannabis use (GROUP) rated as frequency of use in the period of most frequent use on a scale of (never) to 3 (daily), lifetime (baseline) and incident (past three years, follow-up)

L Education level (GROUP); 0 (no education), 1 (primary school), 2-3 (secondary school), 4-5 (highschool), 6 (lower vocational), 7 (higher vocational), 8 (university). 


\section{Analytic strategy}

All analyses were performed using STATA, version $12^{34}$. A priori confounders included in all analyses were age and sex.

\section{Associations of trauma with separate symptoms}

As a first step, it was assessed whether childhood trauma showed stronger associations with any of the symptoms using mixed-effects multilevel regression (XTMELOGIT in STATA) in the NEMESIS- 2 sample. These models allow for a proper test for differences in the way to which trauma impacts on depression, mania, anxiety or psychosis. In other words, instead of analyzing the impact of childhood trauma on each symptom cluster separately, this method tests whether the impact of childhood trauma differs significantly between symptoms ${ }^{35}$. For each subject, four dichotomous outcomes were analyzed, namely the presence of any depressive, manic, anxiety or psychotic symptom. Both the baseline and follow-up measurements were used, accounting for clustering within persons by including a random effect (intercept) at the subject level. Relevant effect sizes (i.e. ratios of odds ratios [RORs]) were obtained by post hoc tests using the LINCOM command. For a more detailed description of this method $\mathrm{see}^{35}$. The analysis was repeated including cannabis use $^{23}$ and education level as covariates.

\section{Associations of trauma with multiple symptoms}

In order to investigate whether childhood trauma shows an increased likelihood of association with multiple symptom domains, rather than association with single symptom domains (e.g. association with depressive symptoms in isolation versus association with depressive symptoms co-occurring with symptoms of mania, psychosis, or anxiety), multinomial logistic regression analyses (MLOGIT command in STATA) were performed, in the general population (NEMESIS-2) sample. The outcome variable consisted of eight categories: (1) no symptoms, (2) depression only, (3) mania only, (4) anxiety only, (5) psychosis only, (6) a combination of two symptom domains (any combination of depression, mania, anxiety, or psychosis), (7) a combination of three symptom domains, (8) all four symptom domains combined. Both the baseline and follow-up measurements were used, while accounting for clustering within persons. Effect sizes were obtained by post hoc tests using the LINCOM command. The analysis was repeated including cannabis use ${ }^{23}$ and education level as covariates.

Similar analyses as described above were performed in a sample of patients with a lifetime mood disorder, a sample of patients with a lifetime anxiety disorder, and in a sample with a (non-affective) psychotic disorder, in order to examine the clinical relevance of reported findings. 
Additional follow-up analyses addressed alternative hypotheses (increased severity or the affective pathway to psychosis) in two different ways. First, it was investigated whether the admixture of symptoms was a consequence of an increase in severity of psychopathology by repeating the analyses while controlling for symptom severity (e.g. associations of childhood trauma with depressive symptoms in isolation versus multiple symptoms, adjusted for number of depressive symptoms). A second follow-up analysis aimed to investigate the earliest stages of psychopathology by repeating the analyses in a subsample of individuals who did not meet criteria for a psychotic, affective or anxiety disorder and did not seek help for their subclinical symptoms $(n=3,986)$. 


\section{RESULTS}

\section{Associations of trauma with separate symptoms}

Table 2 shows the result of the analyses with separate symptoms (i.e. association of trauma with depression or mania or anxiety or psychosis, not necessarily in isolation). Childhood trauma was associated with all symptoms (left column), also after adding cannabis as a covariate (results not shown).

Childhood trauma did show a somewhat stronger association with psychosis than with mania (ROR 1.04, 95\% Cl 1.01-1.06, p=0.006) and anxiety (ROR 1.03, 95\% Cl 1.00-1.05, $\mathrm{p}=0.044)$. However, in the other comparisons (depression versus all other symptoms, anxiety versus mania) no such differences were found (three right hand columns). Adjusting for cannabis or education level did not significantly influence these results (results not shown).

Table 2 Baseline and first follow-up of NEMESIS-2. Associations of childhood trauma and presence of symptoms, and assessment of differences in strength of associations (e.g. whether trauma has a stronger association with one symptom over another)

\begin{tabular}{lllll}
\hline & $\begin{array}{l}\text { Main effect } \\
\text { OR }(95 \% \mathrm{Cl})^{\mathrm{A}}\end{array}$ & $\begin{array}{l}\text { Versus depression } \\
\text { ROR }(95 \% \mathrm{Cl})^{\mathrm{B}}\end{array}$ & $\begin{array}{l}\text { Versus anxiety } \\
\text { ROR }(95 \% \mathrm{Cl})\end{array}$ & $\begin{array}{l}\text { Versus mania } \\
\text { ROR }(95 \% \mathrm{Cl})\end{array}$ \\
\hline Depression $^{\mathrm{C}}$ & $1.21^{* * *}(1.18-1.23)$ & & & \\
Anxiety $^{\mathrm{C}}$ & $1.18^{* * *}(1.16-1.21)$ & $0.99(0.97-1.01)$ & & \\
Mania $^{\mathrm{C}}$ & $1.19 * * *(1.17-1.22)$ & $0.98(0.96-1.00)$ & $1.01(0.99-1.03)$ & $1.04 * *(1.01-1.06)$ \\
Psychosis $^{\mathrm{D}}$ & $1.23^{* * *}(1.20-1.26)$ & $1.02(0.99-1.04)$ & $1.03 *(1.00-1.05)$ & $1.05)$ \\
\hline
\end{tabular}

Note: NEMESIS-2, The second Netherlands Mental Health Survey and Incidence Study; ROR, ratio of odds ratios; Cl, Confidence Interval

All odds ratios (OR) are adjusted for age and sex. Childhood trauma is a combination scale of $0-25$ of 5 different types of trauma; emotional neglect (0 [never]-5[very often]), psychological abuse (0-5), physical abuse (0-5), sexual abuse (0-5) and peer victimization (0-5). (Thus each OR corresponds with a 1 point increase in trauma)

A Odds ratios express association of childhood trauma and presence of symptoms, other symptoms may be present.

${ }^{B}$ Ratios of odds ratios express how much higher/lower the association between symptom and trauma was for each symptom-pair

c Presence of any symptoms. CIDI 3.0 was used for the assessment of depressive, manic or anxiety (social fobia, generalized anxiety or panic disorder) symptoms. A self-constructed questionnaire was used for the assessment of positive psychotic symptoms (self-report)

D Participants that self-reported psychotic symptoms were followed-up with an additional interview performed by a clinician for validation

$* \mathrm{p}<0.05, * * \mathrm{p}<0.01, * * * \mathrm{p}<0.001$

\section{Associations of trauma with multiple symptoms}

\section{General population}

Analysis of the NEMESIS-2 sample revealed a pattern of significantly larger ORs as more symptom clusters were present (Table 3). Thus, childhood trauma was consistently more strongly associated with combinations of symptom domains than with symptoms in isolation. Adjusting for symptom load, cannabis use or education level did not significantly influence these results. Figure 1 shows the group sizes (\%) of subjects with isolated and 
co-occurring symptoms in the general population, divided by non-traumatized and traumatized subjects. Childhood trauma was included as a continuous measure throughout the different analyses but dichotomized for illustrative purposes at the 80th percentile, in accordance with previous work ${ }^{36}$. In Figure 1, a pattern of multiple symptom clusters in traumatized individuals (three right-hand red bars) rather than isolated symptoms (four left-hand red bars) is evident.

The pattern of admixture of symptoms associated with childhood trauma was confirmed in the sample of participants without a mood, anxiety, or psychotic disorder, and without help seeking for mental symptoms (table 3). Childhood trauma was associated with multiple rather then isolated symptoms of all domains. Adjusting for cannabis use or education level did not significantly influence these results (results not shown).

\section{Clinical samples}

The pattern of admixture of multiple symptom domains was also confirmed in the three clinical samples (Table 3). In mood disorder patients, childhood trauma was associated with an increased likelihood of combinations of symptom domains compared with depression in isolation. Results were similar when patients with bipolar disorder were excluded. Similarly, in anxiety disorder, traumatized patients showed an increased likelihood of multiple symptom clusters, compared with anxiety symptoms in isolation.

In the psychotic disorder sample, childhood trauma also showed an increased likelihood of multiple symptom domains, compared with depression, anxiety or psychosis in isolation. In all three clinical samples, adjusting for cannabis use or education level did not significantly influence these results (results not shown). 


\section{DISCUSSION}

\section{Findings}

In a general population sample, childhood trauma was associated with four major mental symptom domains: depression, mania, anxiety and psychosis. The strengths of these associations were similar, except for a somewhat stronger association with psychosis than with anxiety and mania, indicating a central role for reality distortion following childhood trauma, especially since sensitivity analyses suggested that psychotic symptoms are not the 'end-product' of severe affective dysregulation, but rather an integral part of psychopathology following childhood trauma, already present in the earliest stages of psychopathology. Importantly, childhood trauma was associated with multiple symptom clusters rather than with isolated symptoms. As more symptom clusters were combined (i.e. two, three or four symptom clusters) the odds ratios of the association with childhood trauma became significantly larger. The robustness of this pattern of admixture was noteworthy, not affected by controlling for symptom severity, and evident also in the sample of participants specifically selected for the absence of help seeking or a clinical (anxiety, mood or psychotic) disorder. Moreover, the pattern of admixture was consistently found in three different clinical samples.

\section{Why is this important?}

Biological psychiatry aims to understand the pathophysiology of mental disorders. Despite considerable research efforts, however, insights into the origins of mental illness are still limited. Reasons for the limited success may be found in the lack of a biologically informed diagnostic system ('the missing gold standard'), significance chasing with underpowered studies, approximate replications, and extreme comparisons between patients and (super)healthy controls, rather than focusing on clinically relevant subgroups ${ }^{37}$. Kapur and colleagues have recently argued that the application of "stratified medicine" in psychiatry may provide an interesting option to advance our understanding of mental illness. That is: focusing on clinically meaningful differences between homogeneous clinical subtypes across traditional diagnostic categories rather than hypothesis-rejection versus controls ${ }^{37}$. In their paper, this approach is illustrated with an example from the field of oncology: overexpression of human growth factor subtype (HER2) in breast cancer tissue was first identified as a form of breast cancer with a poor prognosis. Using a stratified medicine approach, overexpression of HER2 was later identified in subtypes of several types of cancer. Development of specific therapies considerably increased survival for all these subtypes and now HER2 stratification serves as an important guide for treatment ${ }^{37}$. 


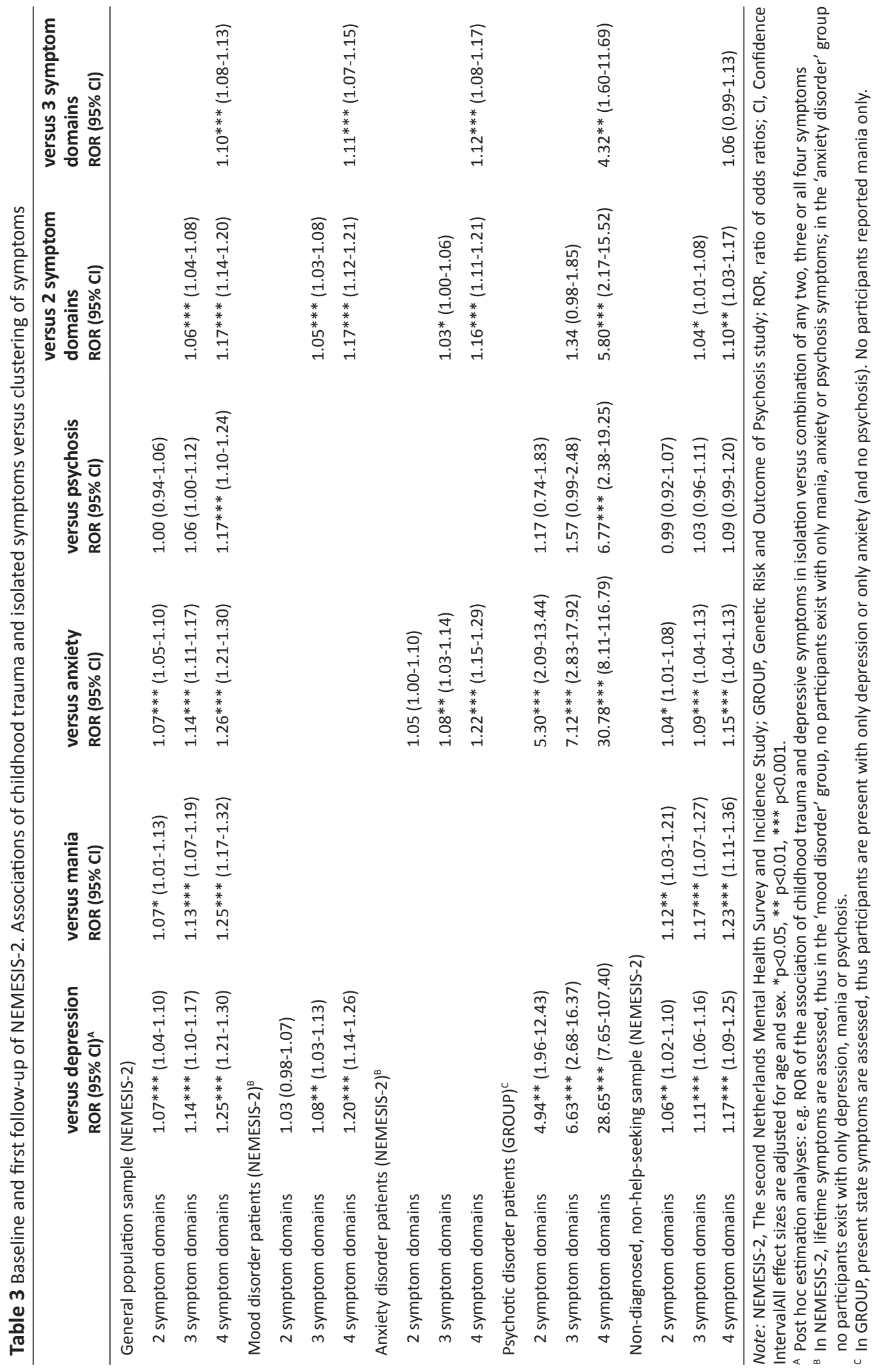


Figure 1 Group sizes (\%) of isolated and co-occurring symptoms in non-traumatized and traumatized subjects in the general population (NEMESIS-2)

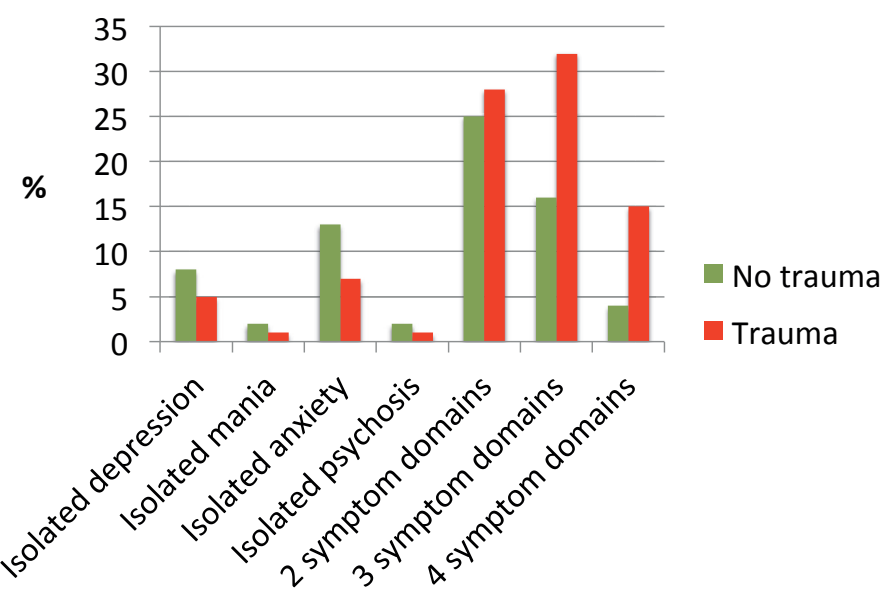

The childhood trauma score was dichotomized at the 80th percentile for illustrative purposes. Traumatized individuals tend to report multiple symptoms (three right hand red bars) rather than isolated symptoms (four left hand red bars).

In this paper, we adopted an environmental stratification approach, showing a specific pattern of symptom admixture across a range of different populations, at the subclinical as well as at the clinical level. These findings may have significant etiological, pathophysiological, diagnostic and clinical repercussion. At the level of etiology, our results strongly support a causal role of childhood trauma in the development of multiple psychiatric disorders, given the specificity of the pattern of admixture of symptoms that was strongly linked to childhood trauma exposure across multiple diagnostic categories.

In addition, these findings should also inform research strategies, as the present results suggest that future work should examine the long-term consequences of childhood trauma across multiple diagnostic domains, rather than focusing on traditional diagnostic categories, in order to elucidate the biological and psychological mechanisms underlying the transition from a subclinical admixture of symptoms, to help seeking, and finally a given clinical disorder. One could infer considerable interplay between the implicated symptom domains ${ }^{14}$, resulting in patterns of psychopathology that are increasingly specific to the individual as symptoms become more severe, and finally result in a diagnosis that to a large degree will depend on which symptoms prevail at that particular point in time ${ }^{38}$. In addition to significant etiological and research implications, these findings should also inform treatment, and possibly, diagnostic decisions. While the exact clinical implications are as yet unclear, active enquiry and diagnostic attention to multiple symptom domains is warranted, rather than only for the domain that is most prominent at the time of presentation. Moreover, once these findings are independently replicated, and further refined in terms of which specific affective, psychotic and anxiety symptoms are involved, it may be argued that these findings should have direct diagnostic repercussions. Finally, the findings also suggest that treatment of traumatic experiences should be considered irrespective of diagnosis, even though this is not always current practice ${ }^{39}$. 
Lastly, development of therapeutic approaches, tailored specifically at individuals exposed to severe childhood traumatic experiences, should be made a priority.

\section{Strengths and limitations}

The current findings should be interpreted in terms of its strengths and limitations. Childhood trauma was assessed by subjective retrospective report, therefore recall bias may have influenced these results, although research suggests good reliability of retrospective assessment of childhood trauma ${ }^{40}$. Childhood trauma was assessed using different questionnaires in the NEMESIS-2 and GROUP studies, thereby diminishing comparability between these samples. However, as the main focus of this study was the investigation of the association between trauma and symptoms, and not the comparison of different groups in terms of trauma history, the difference in methodology may be considered less relevant. As in earlier studies using these samples no clear evidence was found to support differential psychopathological outcomes depending on different childhood traumatic experiences ${ }^{35,41}$, the different types of trauma were grouped to one trauma scale, however additional studies may be warranted investigating differential effects of separate trauma types in more detail. Additionally, a more detailed investigation of gender differences may be warranted in future studies ${ }^{42}$. Even though the baseline and first follow-up measurements of NEMESIS-2 were used, the analyses performed used these measurements as cross-sectional data, since the incidence of novel symptoms in this sample was too low to allow for prospective analyses. Therefore, the present study does not allow to fully address the developmental model suggested by the cross-sectional analyses. However, by performing the various sensitivity analyses in separate subsamples at different stages of psychopathology, we were able to address at least part of this issue. Lifetime presence of symptoms was assessed, precluding assessment of simultaneously occurring symptoms. However, this study aimed to assess development of symptoms over the lifetime rather then at the same point in time, making the assessment of simultaneously occurring symptoms unnecessary.

A further limitation is that we did not have comprehensive data on (complex) post-traumatic stress disorder, borderline personality disorder or dissociative disorder, disorders that are also associated with childhood trauma. Future research should incorporate these disorders as well; however, they are known for their prominent mixture of affective and anxiety symptoms, and psychotic symptoms are often described as well, so we would argue that our findings may also extend to these disorders.

Strengths of this study are the multiple and large samples as well as the representativeness of the samples, increasing the generalizability of these results. By incorporating symptom assessments across diagnostic boundaries this study adds important and more comprehensive evidence to psychiatric research focused on the sequelae of traumatic experiences. Lastly, assessment of symptoms was conducted by trained clinical interviewers in the GROUP study and all reports of psychotic symptoms in the larger NEMESIS-2 study were validated by clinicians, thus increasing reliability compared to self-report measures of particularly psychosis. 
In conclusion, these findings provide evidence for childhood trauma as a major risk factor for psychopathology, characterized by an admixture of affective, anxiety and psychotic symptoms, which may already be present at the earliest stages of psychopathology.

\section{Acknowledgements}

This work was supported by the Ministry of Health, Welfare and Sports (grant number 310253); with supplement support from the Netherlands Organization for Health Research and Development (ZonMw). Supported by the European Community's Seventh Framework Program under grant agreement No.HEALTH-F2-2009-241909 (Project EU-GEI).

Appendix 1 list of depressive symptoms

In the period of several days in which you felt low, did you

1. feel low, empty or depressed on an almost daily basis, for most of the day

2. feel dispirited about life

3. feel desperate about the future

4. lose interest in things that you usually enjoy

5. have trouble getting enjoyment out of pleasant events

6. have an increase or decrease in appetite

7. have trouble sleeping, or did you sleep more than usual

8. have more or less energy than usual

9. move slower or were you restless

10. have slower or erratic thoughts

11 . have trouble concentrating

12. have trouble making decisions

13. lose your self-confidence

14. feel inferior to others

15. feel worthless

16. feel very guilty

17. feel irritable, or in a bad mood

18. feel nervous or anxious

19. get sudden attacks of fear or anxiety

20. think a lot about death

21. think that it would be better if you were dead

22. contemplate ending your life

23. make plans to end your life

24. try to end your life

25. feel like you couldn't handle daily responsibilities

26. prefer to be alone than with family or friends

27. feel less like talking

28. cry a lot 
Appendix 2 list of mania symptoms

Did you have a period of several days in which you

1. were restless, exited or full of energy

2. were irritated or moody

3. were so irritable that you fought with, yelled at or hit people

4. were restless and couldn't stand still

5. had a big increase in sexual appetite, or wanted sex with inappropriate people

6. were too friendly to others

7. did things or talked about things that would usually embarrass you

8. tried to do impossible things, like way too much work

9. were very talkative, or could not stop talking

10. constantly changed plans

11. had trouble concentrating

12. had erratic thoughts

13. needed much less sleep than usual

14. get involved in irresponsible investments

15. spent too much money

16. did reckless things

17. were overly self-confident

18. thought you were someone else, or affiliated with someone famous (which was not true)

Appendix 3 list of anxiety symptoms

- Panic disorder (PD)

1. Did you have an attack of anxiety or panic During this attack, did you

2. have heart palpitations

3. feel short of breath

4. feel nauseous

5. feel dizzy or lightheaded

6. sweat a lot

7. shake or tremble

8. have a dry mouth

9. feel like you were suffocating

10. have chest pains

11. feel like you lost self control

12. feel dissociated

13. feel like your would pass out

14. think you would die

15. get hot or cold flashes

16. feel numb 
17. did you have a period in which you felt very shy or scared with others

Did you feel like this when

18. meeting new people

19. talking to authoritative people

20. talking in public

21. going to social gatherings

22. doing a performance

23. doing an important exam or job interview

24. working in the presence of others

25 . going into a room when others are present

26. talking to people you don't know well

27. talking to people you don't agree with

28. writing, eating or drinking in the presence of others

29. using a public toilet or someone else's bathroom

30. on a romantic date

31. in any other situation when you are the center of attention

- Generalized Anxiety Disorder (GAD)

32. Did you have a period in which you worried more, or were more nervous than others who have similar problems, or

During this period, did you

33. feel restless or tense

34. get tired easily

35. feel irritable

36. have trouble concentrating

37. have tense, sensitive or painful muscles

38. have trouble sleeping

39. have heart palpitations

40. sweat a lot

41. shake or tremble

42. get a dry mouth

43. get down or depressed 
Appendix 3 list of psychosis symptoms

Did you feel that

1. people were spying on you

2. people were following you

3. you were secretly being tested on

4. someone was conspiring against you

5. a 'double' had taken the place of a loved one

6. someone was reading your mind

7. you could hear the thoughts of others

8. others could hear your thoughts

9. alien thoughts were placed in your head

10. someone took thoughts from your head

11. special messages were sent to you through media

12. you were influenced by strange energies

13. you were being controlled by an outer force

14. your thoughts were being influenced by machines

15. any other delusion reported by subject

16. you saw things that no one else could see

17. you could hear things that no one else could hear

18. your own thoughts were broadcasted

19. you smelled strange things, that others could not smell

20. you had strange sensations, like being touched when no one was around 


\section{REFERENCES}

1. Varese F, Smeets F, Drukker M, et al. Childhood adversities increase the risk of psychosis: a meta-analysis of patient-control, prospective- and cross-sectional cohort studies. Schizophrenia Bulletin 2012;38(4):661-671.

2. Nanni V, Uher R, Danese A. Childhood maltreatment predicts unfavorable course of illness and treatment outcome in depression: a meta-analysis. American Journal of Psychiatry 2012;169:141-151.

3. Kuo JR, Goldin PR, Werner K, Heimberg RG, Gross JJ. Childhood trauma and current psychological functioning in adults with social anxiety disorder. Journal of Anxiety Disorders 2011;25(4):467-473.

4. Etain B, Henry R, Bellivier F, Mathieu F, Leboyer M. Beyond genetics: childhood affective trauma in bipolar disorder. Bipolar Disorders 2008;10:867-876.

5. Matheson SL, Shepherd AM, Pinchbeck RM, Laurens KR, Carr VJ. Childhood adversity in schizophrenia: a systematic meta-analysis.

Psychological Medicine 2012;43(2):225-238.

6. Read J, Fosse R, Moskowitz A, Perry B. The traumagenic neurodevelopmental model of psychosis revisited. Neuropsychiatry 2014;4(1):65-79.

7. Vreeburg SA, Hoogendijk WJG, van Pelt J, et al. Major depressive disorder and hypothalamic-pituitary-adrenal axis activity. Results from a large cohort study. Archives of General Psychiatry 2009;66(6):617-626.
8. Elzinga BM, Spinhoven P, Berretty E, de Jong $\mathrm{P}$, Roelofs $\mathrm{K}$. The role of childhood abuse in HPA-axis reactivity in social anxiety disorder: a pilot study. Biological Psychiatry 2010;83(1):1-6.

9. Valiengo LL, Soeiro-de-Souza MG, Marques AH, Moreno DH, Juruena MF, Andreazza AC, Gattaz WF, Machado-Vieira R. Plasma cortisol in first episode drug-naive mania: differential levels in euphoric verus irritable mood. Journal of Affective Disorders 2012;138(1-2):149-152.

10. van Winkel R, Esquivel $G$, Kenis $G$, et al. Genome-wide findings in schizophrenia and the role of gene-environment interplay. CNS Neuroscience \& Therapeutics 2010;16(5):5-e192.

11. Arseneault $L$, Cannon $M$, Fisher $H$, Polanczyk G, Moffitt TE, Caspi A. Childhood trauma and children's emerging psychotic symptoms: a genetically sensitive longitudinal cohort study. American Journal of Psychiatry 2011;168:65-72.

12. Wigman JTW, Van Winkel R, Ormel J, Verhulst FC, Van Os J, Vollebergh WAM. Early trauma and familial risk in the development of the extended psychosis phenotype in adolescence. Acta Psychiatrica Scandinavia 2012;126(4):266-273.

13. Fisher $\mathrm{H}, \mathrm{McGuffin} \mathrm{P}$, Boydell J, et al. Interplay between childhood physical abuse and familial risk in the onset of psychotic disorders. Schizophrenia Bulletin 2014;doi: 10.1093/schbul/ sbt201. 
14. van Os J. The dynamics of subthreshold psychopathology: implications for diagnosis and treatment. American Journal of Psychiatry 2013;170:695-698.

15. Fusar-Poli $P$, Yung AR, McGorry $P$, van Os J. Lessons learned from the psychosis high-risk state: towards a general staging model of prodromal intervention. Psychological Medicine 2014;44:17-24.

16. Smeets $F$, Lataster $T$, Dominguez MG, Hommes J, Lieb R, Wittchen HU, van Os J. Evidence That Onset of Psychosis in the Population Reflects Early Hallucinatory Experiences That Through Environmental Risks and Affective Dysregulation Become Complicated by Delusions. Schizophrenia Bulletin 2012;38(3):531-542.

17. Read J, Hammersley P, Rudegeair T. Why, when and how to ask about childhood abuse. Advances in Psychiatric Treatment 2007;13:101-110.

18. Read J, van Os J, Morrison AP, Ross CA. Childhood trauma, psychosis and schizophrenia: a literature review with theoretical and clinical implications. Acta Psychiatrica Scandinavia 2005;112:330-350.

19. Myin-Germeys I, van Os J. Stressreactivity in psychosis: evidence for an affective pathway to psychosis. Clinical Psychology Review 2007;27:409-424.
20. De Graaf R, Ormel J, Ten Have M, Burger $\mathrm{H}$, Buist-Bouwman M. Mental disorders and service use in the Netherlands. Results from the European Study of the Epidemiology of Mental Disorders (ESEMeD). The WHO World Mental Health Surveys: Global Perspectives on the Epidemiology of Mental Disorders. Kessler, R.C., Üstün, T.B. ed: Cambridge University Press; 2008:388-405.

21. De Graaf $R$, ten Have $M$, van Dorsselaer S. The Netherlands Mental Health Survey and Incidence study-2 (NEMESIS-2): design and methods. International Journal of Methods in Psychiatric Research 2010;19(3):125-141.

22. van Nierop M, van Os J, Gunther N, et al. Does Social Defeat mediate the association between childhood trauma and psychosis? Evidence from the Nemesis-2 study. Acta Psychiatrica Scandinavia 2014;129(6):467-476.

23. Moore THM, Zammit $S$, LingfordHughes A, Barnes TRE, Jones PB, Burke $M$, Lewis $G$. Cannabis use and risk of psychotic or affective mental health outcomes: a systematic review. The Lancet 2007;370(9584):319-328.

24. Harley M, Kelleher I, Clarke M, Lynch F, Arseneault L, Connor D, Fitzpatrick C, Cannon M. Cannabis use and childhood trauma interact additively to increase the risk of psychotic symptoms in adolescence. Psychological Medicine 2010;40:1627-1634. 
25. Andrew G, Peters L. The psychometric properties of the Composite International Diagnostic Interview. Social Psychiatry and Psychiatric Epidemiology 1998;33:80-88.

26. Helzer JE, Robins LN, McEvoy LT, Spitznagel EL, Stoltzman RK, Farmer A, Brockington IF. Physician reexamination of lay-interviewed cases in the general population. Archives of General Psychiatry 1985;42(7):657-666.

27. Bijl RV, van Zessen G, Ravelli A, de Rijk $C$, Langendoen $Y$. The Netherlands Mental Health Survey and Incidence Study (NEMESIS): Objectives and design Social Psychiatry and Psychiatric Epidemiology 1998;33:581-586.

28. Spitzer RL, Williams JB, Gibbon M, First MB. The Structured Clinical Interview for DSM-III-R (SCID). I: History, rationale, and description. Archives of General Psychiatry 1992;49:624-629.

29. Korver N, Quee PJ, Boos HB, Simons C, de Haan L. Genetic Risk and Outcome of Psychosis (GROUP), a multi site longitudinal cohort study focused on gene-environment interaction: objectives, sample characteristics, recruitment and assessment methods. International Journal of Methods in Psychiatric Research 2012;21(3):205-221.

30. Andreasen NC, Flaum M, Arndt S. The comprehensive assessment of symptoms and history (CASH). An instrument for assessing diagnosis and psychopathology. Archives of General Psychiatry 1992;49(8):615-623.
31. Wing JK, Babor T, Brugha $\mathrm{T}$, et al. SCAN. Schedules for Clinical Assessment in Neuropsychiatry. Archives of General Psychiatry 1990;47(6):589-593.

32. Bernstein DP, Stein JA, Newcomb MD, et al. Development and validation of a brief screening version of the Childhood Trauma Questionnaire. Child Abuse \& Neglect 2003;27:169-190.

33. Kay SR, Fiszbein A, Opler LA. The positive and negative syndrome scale (PANSS) for schizophrenia. Schizophrenia Bulletin 1987;13(2):261-276.

34. Statacorp. Stata Statistical Software: Release 12. College Station, TX: StataCorp LP. 2011.

35. van Nierop M, Lataster $\mathrm{T}$, Smeets $\mathrm{F}$, et al. Psychopathological mechanisms linking childhood traumatic experiences to risk of psychotic symptoms. Analysis of a large, representative population-based sample.

Schizophrenia Bulletin 2014;40(suppl.2):S123-S130.

36. Heins $M$, Simons $C$, Lataster $T$, et al. Childhood trauma and psychosis: A case-control and case-sibling comparison across different levels of genetic liability, psychopathology, and type of trauma. American Journal of Psychiatry 2011;168(12):1286-1294.

37. Kapur S, Phillips AG, Insel TR. Why has it taken so long for biological psychiatry to develop clinical tests and what to do about it? Molecular Psychiatry 2012;17:1174-1179. 
38. Wigman JTW, van Os J, Thiery E, Derom C, Collip D, Jacobs N, Wichers M. Psychiatric diagnosis revisited: towards a system of staging and profiling combining nomothetic and idiographic parameters of momentary mental states. Plos One 2013;8(3):e59559.

39. De Bont PA, van den Berg DP, van der Vleugel BM, et al. A multi-site single blind clinical study to compare the effects of prolonged exposure, eye movement desensitization and reprocessing and waiting list on patients with a current diagnosis of psychosis and co morbid post traumatic stress disorder: study protocol for the randomized controlled trial Treating Trauma in Psychosis. Trials 2013;14(151):doi: 10.1186/ 1745-6215-1114-1151.
40. Fisher HL, Craig TK, Fearon P, et al. Reliability and comparability of psychosis patients' retrospective reports of childhood abuse. Schizophrenia Bulletin 2011;37(3):546-553.

41. van Nierop $M$, van Dam $D$, Viechtbauer $W$, Velthorst $E$, van Winkel R, GROUP Investigators. Childhood abuse and neglect in relation to presence and persistence of psychotic and depressive symptomatology. Psychological Medicine 2014;doi:10.1017/ S0033291714001561.

42. Fisher $H$, Morgan $C$, Dazzan $P$, et al. Gender differences in the association between childhood abuse and psychosis. British Journal of Psychiatry 2009;194(4):319-325. 


\title{
CHAPTER 11
}

\section{The Functional and Clinical Relevance of Childhood Trauma-Related Admix- ture of Affective, Anxious, and Psychosis Symptoms: Further Support for a Stratified Medicine Approach in Psychiatry}

\author{
Martine van Nierop ${ }^{A}$ \\ Maarten Bak ${ }^{\mathrm{A}}$ \\ Ron de Graaf \\ Margreet ten Have ${ }^{B}$ \\ Saskia van Dorsselaer ${ }^{B}$ \\ Genetic Risk and OUtcome of Psychosis (GROUP) Investigators* \\ Ruud van Winkel ${ }^{\mathrm{AC}}$
}

\author{
* The GROUP Investigators are: \\ Richard Bruggeman ${ }^{\mathrm{D}}$ \\ Wiepke Cahn ${ }^{\mathrm{E}}$ \\ Lieuwe de Haan ${ }^{\mathrm{F}}$ \\ Rene S. Kahn ${ }^{\mathrm{E}}$ \\ Carin J. Meijer ${ }^{\mathrm{F}}$ \\ Inez Myin-Germeys ${ }^{\mathrm{A}}$ \\ Jim van $\mathrm{Os}^{\mathrm{AG}}$ \\ Durk Wiersma ${ }^{\mathrm{D}}$
}

\footnotetext{
A Maastricht University Medical Centre, Dept. of Psychiatry \& Psychology, School for Mental Health and Neuroscience, Maastricht, The Netherlands

${ }^{B}$ Netherlands Institute of Mental Health and Addiction, Utrecht, The Netherlands

c University Psychiatric Centre, Katholieke Universiteit Leuven, campus Kortenberg, Kortenberg, Belgium

D University Medical Centre Groningen, Dept. of Psychiatry, Groningen, The Netherlands

E University Medical Centre Utrecht, Dept. of Psychiatry, Rudolf Magnus Institute of Neuroscience, Utrecht, The Netherlands

F Academic Medical Centre, University of Amsterdam, Dept. of Psychiatry, Amsterdam, The Netherlands

${ }^{\mathrm{G}}$ King's College London, King's Health Partners, Dept. of Psychosis Studies, Institute of Psychiatry, London, United Kingdom
} 


\section{ABSTRACT}

\section{Background}

Previous work has shown that across samples of patients with mood disorder, anxiety disorder or psychotic disorder, patients with childhood trauma are more likely to have co-occurrence of affective, anxious and psychosis symptoms than non-traumatized patients. These findings suggest that stratification according to childhood trauma may be helpful to identify etiological subgroups. However, the clinical relevance of trauma-related admixture remains to be established.

\section{Methods}

We examined patients with mood disorder (NEMESIS-2; $n=1260$ ), anxiety disorder (NEMESIS-2; $n=896$ ) or psychotic disorder (GROUP; $n=532$ ) in terms of symptom profiles, quality of life and social functioning.

\section{Results}

Results showed that mood disorder patients with both trauma and co-occurrence of affective, anxious and psychosis symptoms had a lower quality of life (B $-12.6,95 \% \mathrm{Cl}$ -17.7- -7.5, $\mathrm{p}<0.001$ ), more help-seeking behaviour (Odds Ratio [OR] 2.5, 95\% Cl 1.1-5.7, $\mathrm{p}=0.031$ ), and higher prevalence of substance use disorders (OR 7.8, 95\% Cl 1.1-58.0, $\mathrm{p}=0.044$ ), compared with patients without trauma history and symptom admixture (CT-/ CL-). Similar results were found in patients with an anxiety disorder. Patients with a psychotic disorder and trauma-related admixture showed lower quality of life (B -0.6, 95\% $\mathrm{Cl}-0.9--0.4, \mathrm{p}<0.001$ ), higher prevalence of drug disorders (OR 2.2, 95\% $\mathrm{Cl} 1.2-3.9$, $\mathrm{p}=0.008$ ), and lower global assessment of functioning ([GAF] B $-12.8,95 \% \mathrm{Cl}-17.1--8.5$, $\mathrm{p}<0.001$ ) than $\mathrm{CT}-/ \mathrm{CL}$ - patients.

\section{Conclusion}

Stratification according to childhood trauma exposure thus identifies a phenotype characterized by admixture of affective, anxiety and psychotic symptoms that has functional and clinical relevance. Identification of functionally meaningful aetiological subgroups may aid clinical practice. 


\section{INTRODUCTION}

Despite efforts to develop valid clinical tests ${ }^{1,2}$, the process of psychiatric diagnosis and treatment still largely depends on observed behaviours and reported experiences. The failure to develop clinical tests is in part related to the fact that diagnostic classification categories, while useful for clinical practice, lack biological and etiological validity ${ }^{3}$. In order to develop a better functional understanding of clinical heterogeneity, and a more informed system for diagnosis and treatment, a more personalised approach, based on transdiagnostic stratification with the aid of experiential, neurobiological and etiological factors may be productive ${ }^{34}$.

Work from our group has shown the viability of such a stratification approach by stratifying according to a known environmental risk factor for psychopathology: childhood trauma ${ }^{5,6}$. Childhood trauma is an important risk factor for several mental disorders such as depression ${ }^{7}$, anxiety disorder ${ }^{8}$ and psychotic disorder ${ }^{9}$, but it remains unclear why some individuals go on to develop depression following childhood trauma while others may develop schizophrenia or a generalized anxiety disorder.

We hypothesized that childhood trauma may not specifically induce a particular disorder, but rather the co-occurrence of symptoms of depression, psychosis, and anxiety over the life course. Depending on which symptoms prevail at any given point, and how they develop over time, diagnoses given to individual patients may vary. Previous work has demonstrated that individuals exposed to early adverse events were more likely to develop an admixture of affective, anxious and psychosis symptoms, rather than any of these symptom clusters in isolation ${ }^{5,6}$. Such an admixture of symptoms was reported in a sample of healthy participants $(n=3,986)$, as well as in samples of patients with a mood disorder $(n=1,260)$, an anxiety disorder $(n=896)$, and a psychotic disorder $(n=532)^{5}$.

While these findings may aid in our understanding of clinical heterogeneity in relation to underlying etiology, the notion of a particular childhood trauma-related phenotype only has relevance to clinicians if it is also associated with functional outcome, and if specific interventions aimed at reducing (the effects of) the etiological factor are available. Previous work has shown that psychological therapies targeting traumatic experiences are feasible, also in patients with psychosis ${ }^{10-13}$. Therefore, efforts to examine the functional and clinical correlates of this particular subgroup may be productive. The present study aimed to examine the three clinical samples used in a previous study (patients with a mood, anxiety and psychotic disorder ${ }^{5}$ ) in terms of functional outcome, stratifying by childhood trauma history, and the associated level of clustering of symptoms. 


\section{METHODS}

\section{Mood disorder and anxiety disorder}

\section{NEMESIS-2 samples}

The first two samples, i.e. participants with a mood disorder and participants with an anxiety disorder, were derived from the ongoing second Netherlands Mental Health Survey and Incidence Study (NEMESIS-2) ${ }^{14}$, a representative general population sample studying the mental health of the Dutch population. For the present analysis, the baseline assessment of NEMESIS-2 was used. The study was approved by the Medical Ethics Review Committee for Institutions on Mental Health Care. Participants aged 18-65 years were included; insufficient fluency in Dutch was an exclusion criterion. Participants were interviewed by trained interviewers, who were not clinicians, with the Composite International Diagnostic Interview (CIDI) version $3.0^{15}$ and additional questionnaires. A more detailed description of NEMESIS-2 methodology was presented by De Graaf and colleagues ${ }^{14}$.

A total number of 6646 subjects participated at the baseline measurement. Of these, two clinical samples were derived: a sample of participants with a mood disorder (major depressive disorder, bipolar disorder or dysthymia, $n=1260$ ) and a sample of participants with an anxiety disorder (social phobia, panic disorder or generalized anxiety disorder, $\mathrm{n}=896$ ). Diagnoses were established using the CIDI 3.0.

\section{Measures}

Symptom categories within mood and anxiety disorder

Symptoms were measured using the CIDI 3.0 (depression, anxiety and mania) and a questionnaire based on the CIDI 1.1 (psychosis). A detailed description can be found in a recent report by Van Nierop and colleagues $\left(2014^{5}\right)$. In that study, a variable for symptom categories was constructed by assessing presence of any the following four symptom clusters, divided over eight categories: 1 (no symptoms), 2 (isolated depression), 3 (isolated mania), 4 (isolated anxiety), 5 (isolated psychosis), 6 (any combination of two of these four symptom clusters), 7 (any combination of three symptom clusters) and 8 (all four symptom clusters). Since associations with childhood trauma were highest in participants with symptoms in at least three clusters ${ }^{5}$, participants were divided into two categories:

(1) no or minimal clustering (i.e. no symptoms, isolated symptoms only, or combinations of maximally two symptom clusters [categories 1-6]; CL-; mood disorder $n=592$ [47\%], anxiety disorder $\mathrm{n}=450[50 \%])$; and

(2) clustering (combinations of at least three symptoms [categories 7 or 8]; $\mathrm{CL}+$, mood disorder $n=668[53 \%]$, anxiety disorder $n=446[50 \%])$. 


\section{Childhood trauma}

Childhood trauma was assessed using a format based on the NEMESIS-1 trauma questionnaire ${ }^{14}$. Whenever a subject reported having experienced one of five types of childhood trauma (emotional neglect, physical abuse, psychological abuse, sexual abuse and peer victimization; before the age of 16), they were asked to state how often it had occurred, on a scale of 1 (once) to 5 (very often). Factor analysis indicated that the five childhood trauma scales loaded onto one factor (eigenvalue 2.47); therefore one childhood trauma scale (range 0-25) was constructed consisting of the sum of the five childhood trauma types, in agreement with previous studies ${ }^{16}$. Following previous work ${ }^{17,18}$, this scale was dichotomized using the 80th percentile of the total trauma score of the full general population sample to 0 (any childhood trauma score under 3; mood disorder $n=781$ [62\%], anxiety disorder $\mathrm{n}=578$ [58\%]; $\mathrm{CT}-$ ) and 1 (any trauma score of 3 or above; $\mathrm{CT}+$ ).

\section{Childhood trauma \& symptom clustering categories}

The childhood trauma and symptom categories were combined to construct one categorical variable describing four different groups:

-0 (no trauma, no clustering; CT-/CL-); mood disorder ( $n=412)$, anxiety disorder ( $n=311)$;

-1 (trauma, no clustering; $C T+/ C L-)$; mood disorder $(n=369)$, anxiety disorder $(n=225)$;

-2 (no trauma, clustering; CT-/CT+); mood disorder ( $n=180)$, anxiety disorder ( $n=139)$;

-3 (trauma, clustering; $C T+/ C L+)$; mood disorder $(n=299)$, anxiety disorder $(n=221)$.

\section{Functioning}

All participants were assessed for employment status and relationship status (married, living with partner). Additionally, categorical level of income (low, middle and high) and level of education (primary, lower secondary, higher secondary and higher professional/ university) was assessed.

Participants were asked if they had, in the previous 12 months, sought help with a family doctor, company doctor, social worker, homecare specialist or mental health specialist for any psychological, drug or alcohol related problems, or whether they have been prescribed any medication for these problems. Any drug or alcohol use disorder (abuse or dependence) was assessed using the CIDI 3.015.

The Short Form (36) Health Survey (SF-36 ${ }^{19}$ ) was used to assess quality of life in terms of physical functioning, social functioning, physical role-functioning, emotional rolefunctioning, mental health, vitality, bodily pain and general health. The SF-36 measures any impairment in these domains in terms of physical or emotional problems. In the current sample, the subscales role-physical and role-emotional showed very low variability within the subgroups, resulting in very large effect sizes not useful for interpretation, thus were omitted from the analyses. 


\section{Psychotic disorder}

\section{GROUP sample}

As the number of participants with a psychotic disorder in NEMESIS-2 was too small $(n=43)$, we used data from an independent sample of patients with a psychotic disorder, the Genetic Risk and Outcome of Psychosis (GROUP) sample ${ }^{20}$. In the GROUP study, patients with a diagnosis of non-affective psychotic disorder were recruited from five university hospitals in the Netherlands and Belgium (Groningen, Amsterdam, Maastricht, Utrecht and Louvain) and their affiliated mental health care institutions. Patients were eligible for inclusion if they: (1) were aged 16-65 years, (2) met the Diagnostic and Statistical Manual of Mental Disorders, Fourth Edition (DSM-IV) criteria for a non-affective psychotic disorder (in Amsterdam, Maastricht, Utrecht and Louvain assessed with the Comprehensive Assessment of Symptoms and History [CASH ${ }^{21}$; in Groningen with the Schedules for Clinical Assessment in Neuropsychiatry [SCAN 2.122]), (3) had first contact with mental health care less than ten years ago, and (4) were proficient in Dutch. Participants were excluded if their estimated level of intelligence was below 70. Participants who were assessed at the Amsterdam, Maastricht, Utrecht and Louvain sites ( $n=532$ ) were used for the present study as this study used symptom data from the CASH, which was not administered at the Groningen site.

\section{Measures}

\section{Symptom categories}

Depressive, anxiety and psychosis symptoms were measured with the PANSS ${ }^{23}$, and mania symptoms with the $\mathrm{CASH}^{21}$. For a detailed description of the measurement of depressive, anxiety, mania and psychosis symptoms see Van Nierop et al. ${ }^{5}$. Childhood trauma/ clustering groups were constructed as described above (see mood disorder and anxiety disorder patients), resulting in the following group sizes: $C T-/ C L-(n=270) ; C T+/ C L-(n=157)$; $C T-/ C T+(n=51)$; and $C T+/ C L+(n=54)$.

\section{Childhood trauma}

Childhood trauma in the GROUP study was assessed with the Dutch version of the Childhood Trauma Questionnaire (CTQ-SF ${ }^{24}$ ), a 25 item self-report questionnaire rated on a scale of 1 (never true) to 5 (very often true). The CTQ measures physical abuse, physical neglect, sexual abuse, emotional abuse and emotional neglect. A total trauma score was obtained by calculating the average of all 25 items. This scale was dichotomized to 0 (low trauma; $n=418,80 \%$ ) and 1 (high trauma; $n=105$ ), using the 80th percentile of trauma scores of controls, conform previous work ${ }^{17,18}$. 


\section{Functioning}

All participants were assessed for employment status, relationship status (married, living with partner), and use of medication. Educational level was assessed using nine categories (1 - no education; 2 - primary school; 3 - lower secondary; 4 - higher secondary; 5 - higher vocational / university). A categorical level of income (low, middle, high) was assessed. For the assessment of symptom severity, the Positive and Negative Syndrome Scale (PANSS ${ }^{23}$ ) was used. The PANSS measures positive psychotic symptoms (7 items), negative psychotic symptoms (7 items), and general psychopathology, such as anxiety, tension and depression (16 items). Each item is scores in terms of severity, ranging from 1 (absent) to 7 (extreme). Scores for the three subscales were analysed as the mean of their respective items. Presence of any substance use disorder (abuse or dependence) was assessed by interview with the Composite International Diagnostic Interview ${ }^{25}$.

The World Health Organization Quality of Life questionnaire (WHOQOL ${ }^{26}$ ) was used to assess quality of life (physical, psychological, social and environmental domains, range 1-5; higher score indicating better functioning). Level of functioning in terms of symptom severity and disability was measured using the Global Assessment of Functioning (GAF ${ }^{27}$, range 0-100; higher score indicating better functioning).

\section{Analyses}

All analyses were performed in Stata, version $13^{28}$, including age and gender as a priori confounders. Separate analyses were performed for each functioning variable described above. The type of analysis (regression, logistic regression, or multinomial regression analysis) depended on the score distribution of each functioning variable (continuous, dichotomous, or categorical). Comparative effect sizes were obtained by performing posthoc analyses using the LINCOM command. 


\section{RESULTS}

\section{Mood disorder patients}

Individuals with trauma-related clustering of symptoms $(\mathrm{CT}+/ \mathrm{CL}+)$ displayed significant lower quality of life and social functioning than individuals without trauma ( $C T-/ C L+)$, without clustering ( $\mathrm{CT}+/ \mathrm{CL}-)$, or without both trauma and clustering (CT-/CL-), (Table 1; Figure 1). The $\mathrm{CT}+/ \mathrm{CL}+$ group had a lower income, was more often unemployed, was more often unfit for work, and less often married, compared with the CT-/CL- group. The $\mathrm{CT}+/$ $\mathrm{CL}+$ group also displayed more help seeking than the other three groups, had a lower educational level, and more often had a substance use disorder, compared with the CT-/ $\mathrm{CL}-$ and $\mathrm{CT}-/ \mathrm{CL}+$ groups.

\section{Anxiety disorder patients}

Within the anxiety disorder patient group, the $\mathrm{CT}+\mathrm{CL}+$ group also displayed lower quality of life and social functioning scores on most of the SF-36 subscales, compared with the three other groups (Table 2; Figure 2). The $\mathrm{CT}+\mathrm{CL}+$ group more often was unemployed, unfit for work, and less often married than the CT-/CL- and CT-/CL+ groups. Compared with the three other groups, the $\mathrm{CT}+\mathrm{CL}+$ group showed more help-seeking behaviour, used more medication for psychological problems, and more often had a substance use disorder. The $\mathrm{CT}+\mathrm{CL}+$ group had a lower income, lower educational level, and more often had an alcohol disorder than the CT-/CL- group.

\section{Psychotic disorder patients}

In the psychotic disorder patient group, the $\mathrm{CT}+\mathrm{CL}+$ group showed lower quality of life as measured with the WHOQOL, and lower functioning (GAF) scores than the CT-/CL- and $\mathrm{CT}-/ \mathrm{CL}+$ groups (Table 3; Figure 3 ). The $\mathrm{CT}+/ \mathrm{CL}+$ group also showed higher PANSS symptom severity scores, had a lower educational level, and was more often in a sheltered living situation than the $\mathrm{CT}-/ \mathrm{CL}$ - and $\mathrm{CT}-/ \mathrm{CL}+$ groups. Compared with the $\mathrm{CT}-/ \mathrm{CL}$ - group, the $\mathrm{CT}+/$ $\mathrm{CL}+$ group had a lower income and more often had a substance use disorder. 


\section{DISCUSSION}

This study shows that trauma-related admixture of symptoms in patients with a mood disorder, an anxiety disorder or a psychotic disorder is associated with clinically meaningful differences in role functioning, quality of life and symptom severity.

These findings are in line with earlier reports of lower functioning in psychiatric patients with a history of childhood trauma ${ }^{29,30}$, or in patients with clustering of symptoms ${ }^{6,31}$. These studies, however, did not take the specific clustering of symptoms associated with exposure to childhood trauma into account. One study did find that patients with an anxiety disorder or depression, who also experienced psychotic symptoms, reported more childhood traumatic experiences ${ }^{6}$. The current findings indicate that within samples of patients with a particular disorder, clinically meaningful differences in functioning are present when considering combinations of symptom clustering and exposure to childhood trauma, the group with worst functioning being the group with childhood trauma-related admixture of multiple symptom categories.

This pattern was less clear in the psychotic disorder patient group, compared with the mood disorder and anxiety disorder patients. One possible explanation for this difference may be because of lower power in these analyses, as the group sizes in the GROUP study were smaller. Additionally, psychotic disorder patients may arguably have a higher clinical severity, compared with individuals with a lifetime mood or anxiety disorder, resulting in lower contrasts in functional outcome when subdividing these patients in groups according to trauma history and symptom clustering. Furthermore, in the NEMESIS-2 study, lifetime symptoms were assessed, whereas in the GROUP study present state symptoms were measured. Nevertheless, despite a smaller number of significant associations with functional outcomes, all associations consistently were in the expected direction.

These findings may be an important addition to the current debate on using a stratified medicine approach in psychiatric research and practice ${ }^{3,32-36}$. Teicher and Samson describe a distinct subtype across diagnostic categories, associated with a childhood trauma history, as an 'ecophenotype', or phenotypic specialization resulting from environmental exposure ${ }^{35}$. In this review, similar patterns in terms of earlier disease onset, greater clinical severity, lower response to treatment and similar brain abnormalities in traumatized individuals across several mental disorders such as depression and anxiety disorders are highlighted. One viable underlying mechanism that may explain these similarities across mental disorders may be found in the field of epigenetics ${ }^{37}$. Environmental exposures, occurring in a critical period during development, such as childhood traumatic experiences, result in genome-wide alterations of DNA methylation patterns, leading to somatic and mental health problems later in life ${ }^{37}$. Thus, stratification according to trauma history is already widely applied in epigenetic studies, with reports of differences in DNA methylation levels and very low overlap in transcriptional profiles when comparing traumatized to non-traumatized patients $s^{38-40}$.

Yet how can these findings be applied to clinical practice? Patients with a trauma-related admixture of symptoms may be more resistant to treatment, demonstrated by lower 
functional outcome. These findings may provide an important indicator for targeted treatment approaches in the form of specific efforts to counter the detrimental effects of traumatic experiences. Further studies specifically aimed at assessing effectiveness of trauma-related treatment in adults, such as trauma-focused cognitive behavioural therapy ${ }^{41}$, outside the context of post-traumatic stress disorder (PTSD) or other trauma related anxiety disorders, are urgently required. One study, recently initiated, is investigating the safety and efficacy of prolonged exposure (PE) and eye movement desensitization (EMDR) in psychotic disorder patients with comorbid PTSD ${ }^{12}$, with promising results ${ }^{13}$.

The question whether childhood trauma has a causal association with an admixture of symptoms, or with a lower functional outcome, cannot fully be addressed in the present study, due to the cross-sectional nature of the data. NEMESIS-2 and GROUP are both longitudinal studies, with follow-up measurements currently underway; therefore part of this issue may be addressed once these studies are completed. It is, however, also possible that some of this association was a result of reverse causality (i.e. individual differences causing childhood traumatic events) ${ }^{42}$. Careful assessment of psychopathological profiles, personality styles in addition to trauma assessment in samples of children, using longitudinal designs, are required to disentangle these questions. Nevertheless, Kelleher and colleagues recently reported attenuation of psychotic experiences in children after cessation of abusive events, arguing for a causal association between trauma and psychosis ${ }^{43}$. Using such a design, further evidence for a causal association between trauma and an admixture of symptoms or functional outcome (e.g. school performance or social functioning) may also be found. Additionally, if these findings are replicated in samples of children, this may provide important avenues for secondary prevention, as arguably any trauma-related treatment may be more effective when applied shortly after exposure, rather than 10 or 20 years later.

\section{Strengths and weaknesses}

The findings should be interpreted in terms of their relative strengths and weaknesses. The samples of mood disorder and anxiety disorder patients were partly overlapping ( $n=466$, i.e. $37 \%$ of mood disorder patients also had an anxiety disorder, and vice versa, $48 \%$ of anxiety disorder patients also had a mood disorder). Comprehensive data on posttraumatic stress disorder (PTSD) and borderline personality disorder (BPD) were not available in these samples, however as these disorders are known for their co-occurrence of symptoms, we think it is likely that results may be generalized to these disorders as well. Moreover, etiological stratification is particularly helpful in disorders for which considerable subsets were exposed to the stratifying factor, but not the vast majority (as is the case in PTSD and BPD), thus allowing for the identification of clinically meaningful subtypes $^{36}$. The NEMESIS-2 and GROUP studies utilized different questionnaires for the assessment of childhood trauma, symptoms and functional outcomes, diminishing the comparability between the mood disorder and anxiety disorder patients on the one hand, and psychotic disorder patients on the other. 
The strengths of this study are the generalizability of these results across different patient groups, as samples of mood disorder, anxiety disorder and psychotic disorder patients were included. In both the NEMESIS- 2 and GROUP study, participants were assessed on a wide variety of functional outcome measures, including validated measures often used in clinical settings.

In conclusion, stratification according to childhood trauma exposure identifies a phenotype characterized by admixture of affective, anxiety and psychotic symptoms that has functional and clinical relevance. These findings show that adopting a 'stratified medicine' approach may aid in distinguishing etiological and clinically meaningful subgroups in mental health research and practice.

\section{Acknowledgements}

Supported by the Geestkracht program of the Dutch Health Research Council (ZON-MW, grant number 10-000-1002), and the European Community's Seventh Framework Program under grant agreement No. HEALTH-F2-2009-241909 (Project EU-GEI). NEMESIS-2 is conducted by the Netherlands Institute of Mental Health and Addiction (Trimbos Institute) in Utrecht. Financial support has been received from the Ministry of Health, Welfare and Sport, with supplementary support from the Netherlands Organization for Health Research and Development (ZonMw) and the Genetic Risk and Outcome of Psychosis (GROUP) investigators. 
Table 1 Associations between childhood trauma and symptom clustering combinations with functioning in mood disorder patients

\begin{tabular}{|c|c|c|c|}
\hline & $C T+/ C L+(n=299)$ & $C T+/ C L+(n=299)$ & $C T+/ C L+(n=299)$ \\
\hline Group comparison: & Versus & Versus & Versus \\
\hline \multirow[t]{2}{*}{ Odds ratios indicate: } & $\begin{array}{l}\text { Association of trauma and } \\
\text { clustering with functioning } \\
\text { (versus absence of both) }\end{array}$ & $\begin{array}{l}\text { Association of trauma with } \\
\text { functioning in patients } \\
\text { with clustering of } \\
\text { symptoms }\end{array}$ & $\begin{array}{l}\text { Association of clustering } \\
\text { with functioning in } \\
\text { patients with trauma } \\
\text { history }\end{array}$ \\
\hline & $O R^{A} / B^{B}(95 \% \mathrm{Cl})$ & OR/B $(95 \% \mathrm{Cl})$ & OR/B $(95 \% \mathrm{Cl})$ \\
\hline \multicolumn{4}{|l|}{ Work / income / education } \\
\hline Unemployed/disabled (OR) & $2.27 * *(1.40-3.69)$ & $1.32(0.86-2.02)$ & $1.04(0.63-1.70)$ \\
\hline Unfit for work (OR) & $3.13 * * *(1.84-5.33)$ & $1.80 *(1.10-2.94)$ & $1.45(0.82-2.53)$ \\
\hline $\begin{array}{l}\text { Low income (vs middle or high } \\
\text { income) (OR) }\end{array}$ & $1.65 * *(1.18-2.31)$ & $1.37(0.98-1.92)$ & $1.04(0.70-1.55)$ \\
\hline Education level (B) & $-0.30 *(-0.57--0.03)$ & $0.03(-0.25-0.31)$ & $-0.42 *(-0.76--0.07)$ \\
\hline \multicolumn{4}{|l|}{ Relationships } \\
\hline Married (OR) & $0.66 *(0.48-0.91)$ & $0.64 * *(0.46-0.88)$ & $1.22(0.82-1.81)$ \\
\hline Living together (OR) & $0.62(0.37-1.03)$ & $0.64(0.38-1.09)$ & $0.67(0.36-1.25)$ \\
\hline \multicolumn{4}{|l|}{ Clinical features } \\
\hline Care use $<12$ months $^{\mathrm{C}}(\mathrm{OR})$ & $3.76 * * *(2.59-5.45)$ & $1.83 * * *(1.30-2.56)$ & $1.62 *(1.08-2.45)$ \\
\hline Meds use $<12$ months $^{\mathrm{D}}(\mathrm{OR})$ & $2.27 * * *(1.56-3.31)$ & $1.41(0.99-2.02)$ & $1.67 *(1.07-2.62)$ \\
\hline $\begin{array}{l}\text { Any drug disorder } \\
\text { (dependence/abuse) (OR) }\end{array}$ & $3.63^{* * *}(2.16-6.11)$ & $2.80 * * *(1.72-4.55)$ & $3.14 * *(1.54-6.41)$ \\
\hline $\begin{array}{l}\text { Any alcohol disorder } \\
\text { (dependence/abuse) (OR) }\end{array}$ & $1.86 * *(1.23-2.80)$ & $1.70 *(1.13-2.55)$ & $1.25(0.75-2.08)$ \\
\hline \multicolumn{4}{|c|}{ Short Form (36) Health Survey (SF-36) } \\
\hline SF-36 physical functioning (B) & $-20.24 * * *(-25.92--14.56)$ & $-9.60 * *(-15.22--3.98)$ & $-11.01 * *(-17.88--4.14)$ \\
\hline SF-36 social functioning (B) & $-30.62 * * *(-37.69--23.55)$ & $-19.63 * * *(-26.59--12.68)$ & $-14.29 * *(-22.77--5.82)$ \\
\hline SF-36 mental health (B) & $-13.69 * * *(-16.21--11.16)$ & $-7.62 * * *(-10.20--5.04)$ & $-8.20 * * *(-11.35--5.06)$ \\
\hline SF-36 vitality (B) & $-13.46 * * *(-16.42--10.51)$ & $-7.66 * * *(-10.68--4.64)$ & $-5.27 * *(-8.95--1.59)$ \\
\hline SF-36 bodily pain (B) & $-21.29 * * *(-28.15--14.43)$ & $-10.95 * *(-17.84--4.07)$ & $-15.17 * * *(-23.64--6.70)$ \\
\hline SF-36 general health (B) & $-12.14 * * *(-15.07--9.20)$ & $-6.54 * * *(-9.54--3.54)$ & $-7.88 * * *(-11.54--4.21)$ \\
\hline
\end{tabular}

Note: $\mathrm{Cl}$, Confidence Interval; * $\mathrm{p}<0.05, * * \mathrm{p}<0.01, * * * \mathrm{p}<0.001$

A OR (Odds Ratio) for dichotomous functioning variables. All significant (in bold) ORs below 1 for functioning variables where a higher score indicates better functioning. All significant (in bold) ORs above 1 for functioning variables where higher score indicates worse functioning.

${ }^{\text {B }}$ B coefficient for continuous variables. All significant (in bold) B coefficients below 0 for functioning variables where a higher score indicates better functioning. All significant (in bold) B coefficients above 0 for functioning variables where higher score indicates worse functioning.

c Any help seeking for psychological, drug or alcohol problems in the past 12 months, with family doctor, company doctor, social worker, homecare, mental health specialist.

${ }^{\mathrm{D}}$ Any medication prescribed for psychological, drug or alcohol problems. 
Table 2 Associations between childhood trauma and symptom clustering combinations with functioning in anxiety disorder patients

\begin{tabular}{|c|c|c|c|}
\hline & $C T+/ C L+(n=221)$ & $C T+/ C L+(n=221)$ & $C T+/ C L+(n=221)$ \\
\hline \multirow[t]{2}{*}{ Group comparisons: } & Versus & Versus & Versus \\
\hline & CT-/CL- (n=311) & CT-/CL+ (n=139) & $C T+/ C L-(n=225)$ \\
\hline \multirow[t]{2}{*}{ Odds ratios indicate: } & $\begin{array}{l}\text { Association of trauma and } \\
\text { clustering with functioning } \\
\text { (versus absence of both) }\end{array}$ & $\begin{array}{l}\text { Association of trauma with } \\
\text { functioning in patients } \\
\text { with clustering of } \\
\text { symptoms }\end{array}$ & $\begin{array}{l}\text { Association of clustering } \\
\text { with functioning in } \\
\text { patients with trauma } \\
\text { history }\end{array}$ \\
\hline & $\mathrm{OR}^{\mathrm{A}} / \mathrm{B}^{\mathrm{B}}(95 \% \mathrm{Cl})$ & OR/B (95\% Cl) & OR/B (95\% Cl) \\
\hline \multicolumn{4}{|l|}{ Work / income / education } \\
\hline Unemployed/disabled (OR) & $4.07 * * *(2.23-7.44)$ & $1.89 *(1.09-3.28)$ & $0.97(0.56-1.68)$ \\
\hline Unfit for work (OR) & $5.02 * * *(2.57-9.80)$ & $2.60 * *(1.39-4.88)$ & $1.38(0.75-2.56)$ \\
\hline $\begin{array}{l}\text { Low income (vs middle or high } \\
\text { income) (OR) }\end{array}$ & $2.43^{* * *}(1.63-3.61)$ & $1.32(0.88-1.97)$ & $1.04(0.66-1.62)$ \\
\hline Education level (B) & $-0.35 *(-0.66--0.03)$ & $0.05(-0.29-0.38)$ & $-0.45 *(-0.85--0.04)$ \\
\hline \multicolumn{4}{|l|}{ Relationships } \\
\hline Married (OR) & $0.42 * * *(0.29-0.61)$ & $0.59 *(0.40-0.89)$ & $0.88(0.56-1.39)$ \\
\hline Living together (OR) & $0.46 * *(0.27-0.79)$ & $0.80(0.45-1.43)$ & $1.67(0.78-3.57)$ \\
\hline \multicolumn{4}{|l|}{ Clinical features } \\
\hline Care use $<12$ months $^{\mathrm{C}}(\mathrm{OR})$ & $5.58 * * *(3.56-8.74)$ & $1.89 * *(1.26-2.82)$ & $2.14 * *(1.33-3.43)$ \\
\hline Meds use $<12$ months $^{\mathrm{D}}(\mathrm{OR})$ & $4.63 * * *(2.87-7.48)$ & $1.80^{* *}(1.17-2.78)$ & $2.96 * * *(1.70-5.16)$ \\
\hline $\begin{array}{l}\text { Any drug disorder } \\
\text { (dependence/abuse) (OR) }\end{array}$ & $4.80 * * *(2.70-8.55)$ & $2.34 * *(1.39-3.93)$ & $4.30 * * *(2.02-9.15)$ \\
\hline $\begin{array}{l}\text { Any alcohol disorder } \\
\text { (dependence/abuse) (OR) }\end{array}$ & $2.01 * *(1.27-3.19)$ & $1.31(0.82-2.07)$ & $1.50(0.86-2.61)$ \\
\hline \multicolumn{4}{|l|}{ Short Form (36) Health Survey } \\
\hline SF-36 physical functioning (B) & $-17.43 * * *(-23.77--11.08)$ & $-5.56(-12.16-1.04)$ & $-7.59 *(-15.16--0.03)$ \\
\hline SF-36 social functioning (B) & $-31.71 * * *(-39.57--23.85)$ & $-15.66 * * *(-23.76--7.57)$ & $-16.52 * *(-25.84--7.19)$ \\
\hline SF-36 mental health (B) & $-12.03 * * *(-14.87--9.18)$ & $-5.71 * * *(-8.77--2.66)$ & $-7.68^{* * *}(-11.18--4.18)$ \\
\hline SF-36 vitality (B) & $-12.18^{* * *}(-15.48--8.87)$ & $-3.25(-6.80-0.30)$ & $-5.41 * *(-9.48--1.34)$ \\
\hline SF-36 bodily pain (B) & $-18.64 * * *(-26.63--10.65)$ & $-11.15^{*}(-19.67--2.63)$ & $-10.06 *(-19.78--0.33)$ \\
\hline SF-36 general health (B) & $-12.09 * * *(-15.45--8.74)$ & $-5.93 * *(-9.53--2.33)$ & $-7.57 * * *(-11.69--3.44)$ \\
\hline
\end{tabular}

Note: $\mathrm{Cl}$, Confidence Interval; ${ }^{*} \mathrm{p}<0.05,{ }^{*} \mathrm{p}<0.01,{ }^{* * *} \mathrm{p}<0.001$

A Odds Ratio for dichotomous functioning variables. All significant (in bold) ORs below 1 for functioning variables where a higher score indicates better functioning. All significant (in bold) ORs above 1 for functioning variables where higher score indicates worse functioning.

${ }^{\text {B }}$ B coefficient for continuous variables. All significant (in bold) B coefficients below 0 for functioning variables where a higher score indicates better functioning. All significant (in bold) B coefficients above 0 for functioning variables where higher score indicates worse functioning.

c Any help seeking for psychological, drug or alcohol problems in the past 12 months, with family doctor, company doctor, social worker, homecare, mental health specialist.

${ }^{D}$ Any medication prescribed for psychological, drug or alcohol problems. 
Table 3 Associations between childhood trauma and symptom cluster combinations with functioning in psychotic disorder patients

\begin{tabular}{|c|c|c|c|}
\hline Group comparisons: & $\begin{array}{l}C T+/ C L+(n=54) \\
\text { versus } \\
C T-/ C L-(n=270)\end{array}$ & $\begin{array}{l}C T+/ C L+(n=54) \\
\text { versus } \\
C T-/ C L+(n=51)\end{array}$ & $\begin{array}{l}C T+/ C L+(n=54) \\
\text { versus } \\
C T+/ C L-(n=157)\end{array}$ \\
\hline \multirow[t]{2}{*}{ Odds ratios indicate: } & $\begin{array}{l}\text { Association of trauma and } \\
\text { clustering with functioning } \\
\text { (versus absence of both) }\end{array}$ & $\begin{array}{l}\text { Association of trauma with } \\
\text { functioning in patients } \\
\text { with clustering of } \\
\text { symptoms }\end{array}$ & $\begin{array}{l}\text { Association of clustering } \\
\text { with functioning in } \\
\text { patients with trauma } \\
\text { history }\end{array}$ \\
\hline & $\mathrm{OR}^{A} / \mathrm{B}^{\mathrm{B}}(95 \% \mathrm{Cl})$ & OR/B (95\% Cl) & OR/B (95\% Cl) \\
\hline \multicolumn{4}{|l|}{ Work / income / education } \\
\hline No job (OR) & $1.06(0.46-2.41)$ & $1.37(0.56-3.35)$ & $1.11(0.36-3.39)$ \\
\hline Unfit for work (OR) & $1.10(0.51-2.36)$ & $1.62(0.70-3.76)$ & $1.19(0.41-3.48)$ \\
\hline $\begin{array}{l}\text { Low income (vs middel or high } \\
\text { income) (OR) }\end{array}$ & $5.02 *(1.44-17.50)$ & $3.58(0.98-13.09)$ & $5.25 *(1.33-20.73)$ \\
\hline Education level (B) & $-0.81 * *(-1.36--0.27)$ & $-0.55(-1.13-0.02)$ & $-0.36(-1.08-0.35)$ \\
\hline \multicolumn{4}{|l|}{ Relationships / living situation } \\
\hline Married (OR) & $0.24(0.05-1.14)$ & $0.29(0.06-1.47)$ & $0.96(0.15-6.06)$ \\
\hline Living together (OR) & $0.61(0.18-2.01)$ & $0.64(0.18-2.28)$ & $1.03(0.24-4.36)$ \\
\hline Sheltered living (OR) & $3.53 * *(1.64-7.59)$ & $2.92 *(1.29-6.59)$ & $2.60(0.83-8.13)$ \\
\hline \multicolumn{4}{|c|}{ Positive And Negative Syndrome Scale (PANSS) symptom severity } \\
\hline PANSS positive severity (B) & $0.98 * * *(0.77-1.19)$ & $0.44 * * *(0.21-0.66)$ & $0.70 * * *(0.42-0.98)$ \\
\hline PANSS negative severity (B) & $0.42 * *(0.17-0.66)$ & $0.06(-0.20-0.32)$ & $0.23(-0.10-0.55)$ \\
\hline PANSS general severity (B) & $0.80 * * *(0.67-0.93)$ & $0.27^{* * *}(0.14-0.41)$ & $0.68 * * *(0.51-0.85)$ \\
\hline \multicolumn{4}{|l|}{ Clinical status } \\
\hline $\begin{array}{l}\text { Any drug disorder (abuse/ } \\
\text { dependence) (OR) }\end{array}$ & $2.45^{* *}(1.31-4.60)$ & $1.86(0.96-3.60)$ & $1.09(0.47-2.51)$ \\
\hline $\begin{array}{l}\text { Current use of antipsychotics } \\
\text { (OR) }\end{array}$ & $0.84(0.34-2.09)$ & $0.61(0.23-1.65)$ & $0.99(0.32-3.07)$ \\
\hline $\begin{array}{l}\text { Current use of other } \\
\text { medication (OR) }\end{array}$ & $1.03(0.50-2.13)$ & $1.36(0.62-2.95)$ & $0.97(0.37-2.54)$ \\
\hline \multicolumn{4}{|c|}{ Global Assessment of Functioning (GAF) } \\
\hline GAF symptoms (B) & $-13.92 * * *(-18.57--9.28)$ & $-4.39(-9.31-0.53)$ & $-11.13 * * *(-17.31--4.96)$ \\
\hline GAF disability (B) & $-12.23 * * *(-16.92--7.53)$ & $-3.54(-8.50-1.42)$ & $-8.18 *(-14.40--1.96)$ \\
\hline \multicolumn{4}{|c|}{ WHO Quality of Life (WHOQOL) } \\
\hline WHOQOL physical (B) & $-0.55 * * *(-0.75--0.36)$ & $-0.10(-0.30-0.10)$ & $-0.24(-0.49-0.02)$ \\
\hline WHOQOL psychological (B) & $-0.62 * * *(-0.81--0.43)$ & $-0.02(-0.22-0.18)$ & $-0.29 *(-0.55--0.04)$ \\
\hline WHOQOL social (B) & $-0.61 * * *(-0.87--0.35)$ & $-0.26(-0.53-0.02)$ & $-0.24(-0.58-0.10)$ \\
\hline WHOQOL environment (B) & $-0.64 * * *(-0.82--0.45)$ & $-0.36 * * *(-0.55--0.16)$ & $-0.37 * *(-0.62--0.12)$ \\
\hline
\end{tabular}

Note: $\mathrm{Cl}$, Confidence Interval; WHO, World Health Organization; * $\mathrm{p}<0.05,{ }^{* *} \mathrm{p}<0.01, * * * \mathrm{p}<0.001$

A Odds Ratio for dichotomous functioning variables. All significant (in bold) ORs below 1 for functioning variables where a higher score indicates better functioning; all significant (in bold) ORs above 1 for functioning variables where higher score indicates worse functioning.

${ }^{\text {B }}$ B coefficient for continuous variables. All significant (in bold) B coefficients below 0 for functioning variables where a higher score indicates better functioning. All significant (in bold) B coefficients above 0 for functioning variables where higher score indicates worse functioning. 
Figure 1 Social and clinical functioning in mood disorder patients, grouped by trauma and symptom clustering combinations

\section{1a. Work situation / income (\%)}

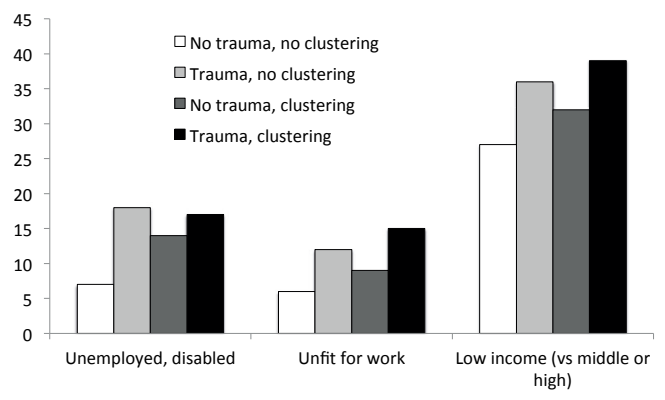

1c. Relationships (\%)

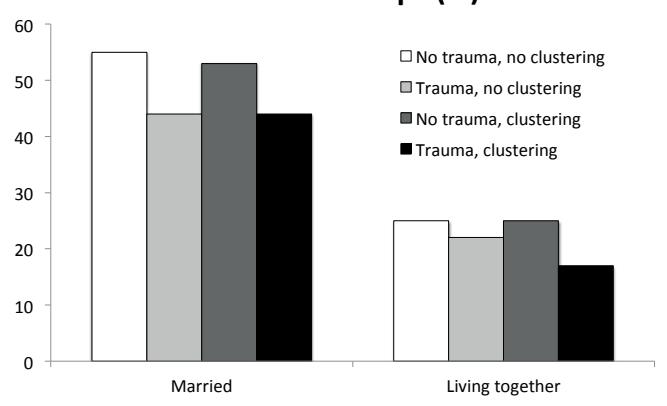

1e. SF-36* (means)

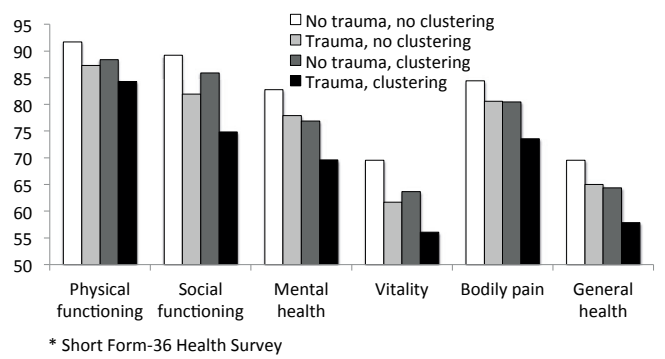

\section{1b. Education level (means)}
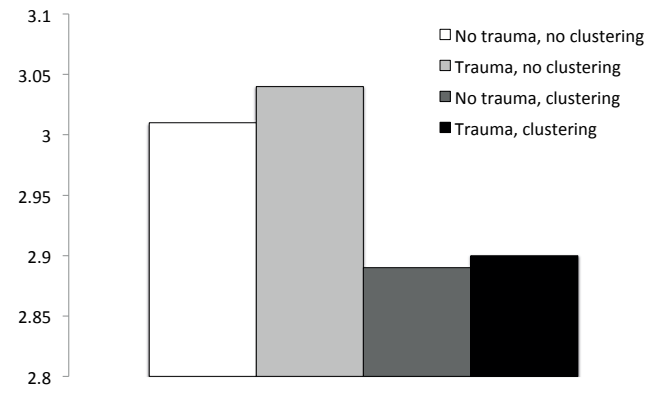

\section{1d. Clinical features (\%)}

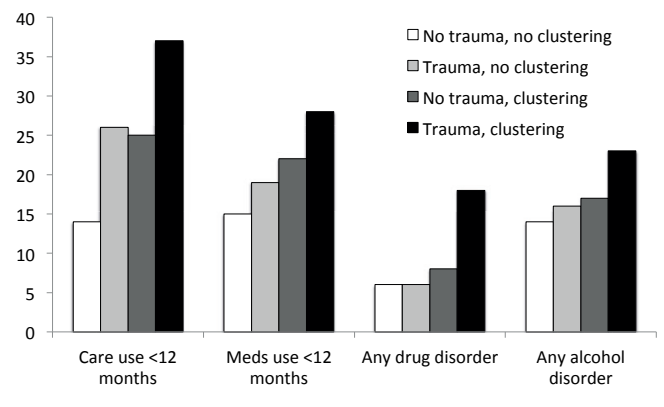


Figure 2 Social and clinical functioning in anxiety disorder patients, grouped by trauma and symptom clustering combinations

2a. Work situation / income (\%)

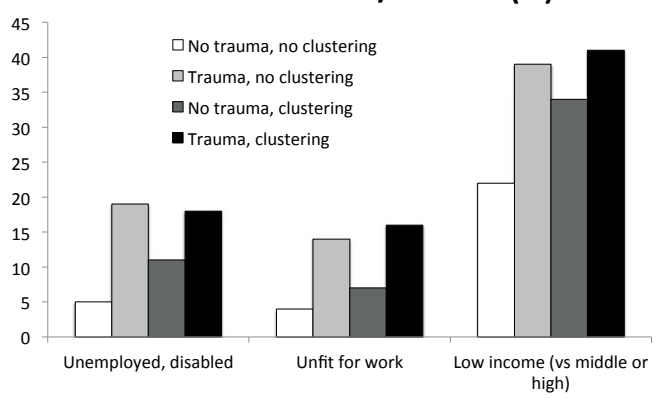

2c. Relationships (\%)

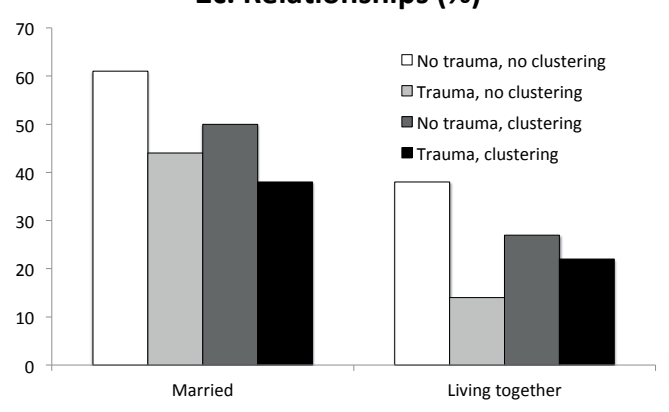

2e. SF-36* (means)

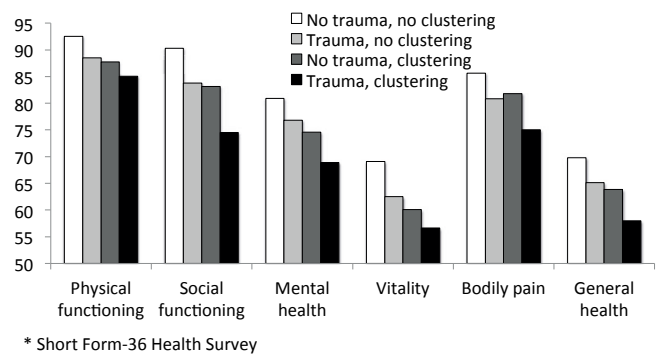

\section{2b. Education level (means)}
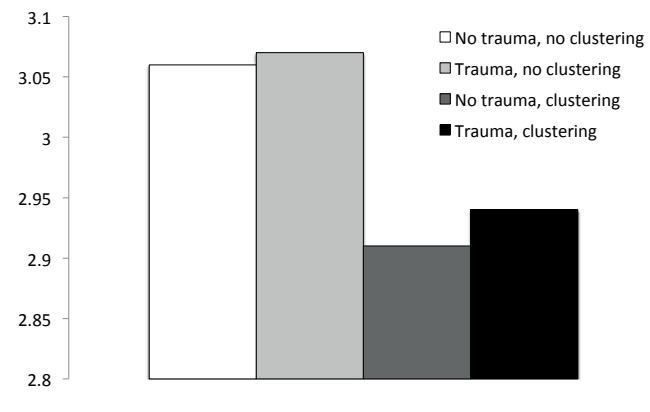

2d. Clinical features (\%)

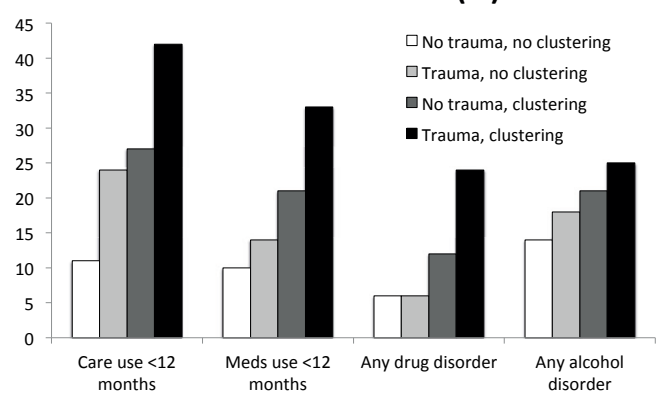


Figure 3 Social and clinical functioning in psychotic disorder patients, grouped by trauma and symptom clustering combinations

\section{3a. Work situation / income (\%)}

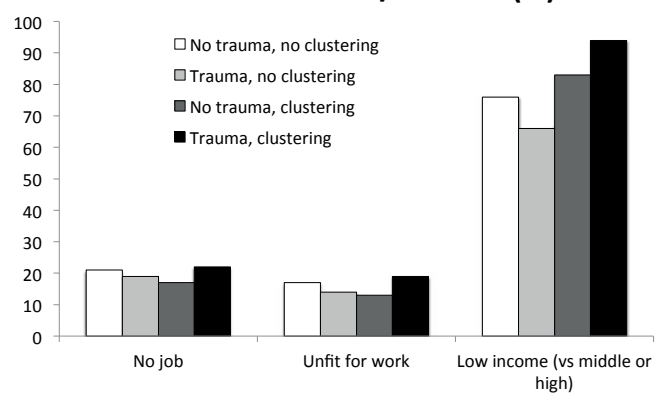

\section{3c. Relationships / living situation (\%)}

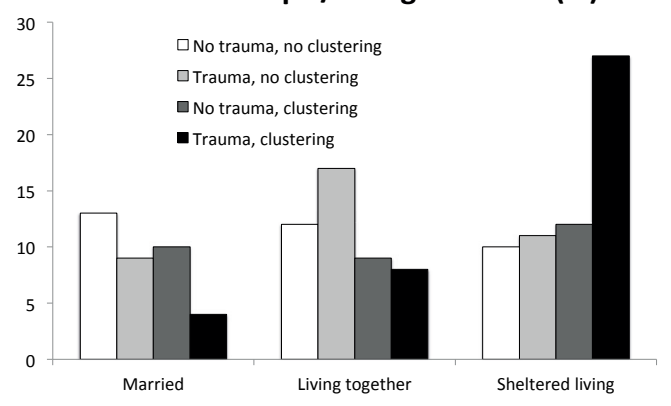

\section{3b. Education level (means)}

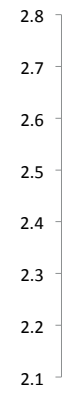

$\square$ No trauma, no clustering

$\square$ Trauma, no clustering

$\square$ No trauma, clustering

- Trauma, clustering

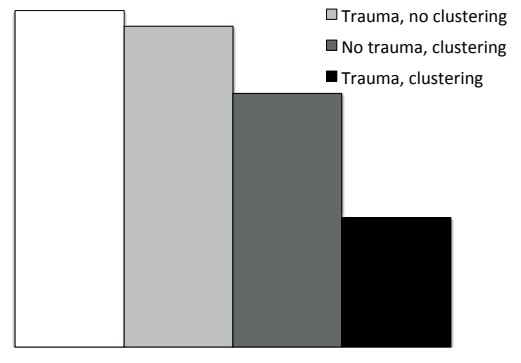

3 3d. Symptoms severity PANSS* (means)

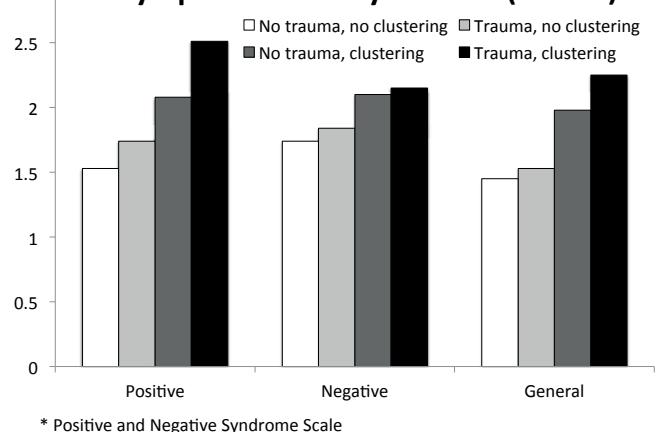


Figure 3 Continued

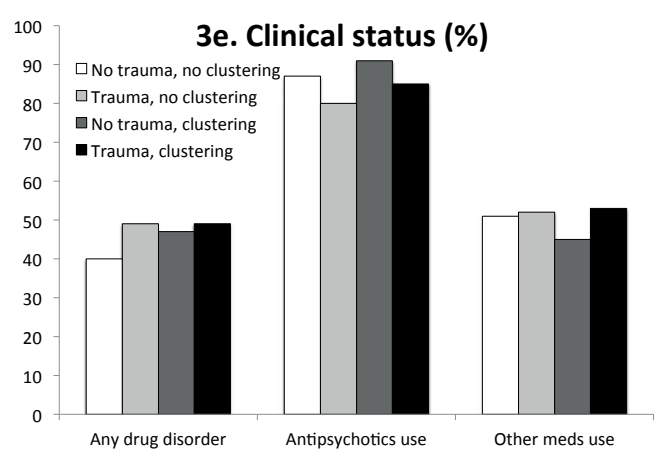

\section{3f. Global Assessment of Functioning (mean:}

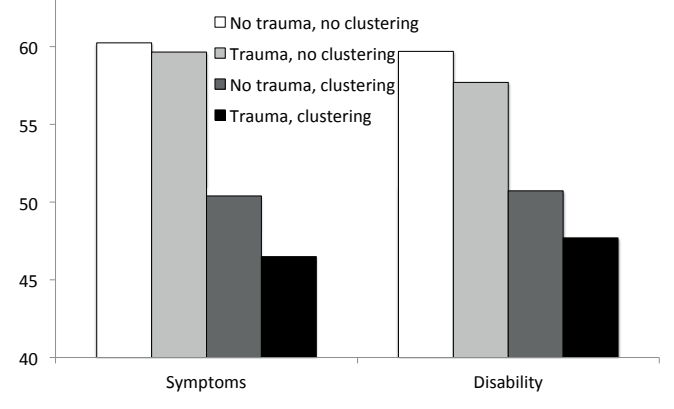

3g. WHO* Quality of Life (means)

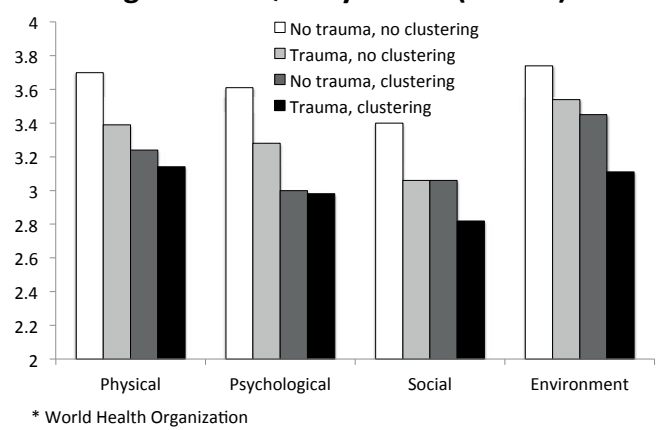




\section{REFERENCES}

1. Prata D, Mechelli A, Kapur S. Clinically meaningful biomarkers for psychosis: A systematic and quantitative review. Neurosci biobehav rev May 272014.

2. Graham J, Salimi-Khorshidi G, Hagan C, Walsh N, Goodyer I, Lennox B, Suckling J. Meta-analytic evidence for neuroimaging models of depression: state or trait? J Affect Disord Nov 2013;151(2):423-431.

3. Kapur S, Phillips AG, Insel TR. Why has it taken so long for biological psychiatry to develop clinical tests and what to do about it? Mol Psychiatr 2012;17:1174-1179.

4. van Os J, Delespaul P, Wigman J, Myin-Germeys I, Wichers M. Beyond DSM and ICD: introducing "precision diagnosis" for psychiatry using momentary assessment technology.

World Psychiatry Jun 2013;12(2):113117.

5. van Nierop M, Viechtbauer W, Gunther N, et al. Childhood trauma is associated with a specific admixture of affective, anxiety, and psychotic symptoms cutting across traditional diagnostic boundaries. Psychol Med 2014; In press.

6. Wigman JTW, van Nierop $M$, Vollebergh W, Lieb R, Beesdo-Baum K, Wittchen HU, Van Os J. Evidence that psychotic symptoms are prevalent in disorders of anxiety and depression, impacting on illness onset, risk, and severity - implications for diagnosis and ultra-high risk research. Schizophr Bull 2012;38(2):247-257.

7. Nanni V, Uher R, Danese A. Childhood maltreatment predicts unfavorable course of illness and treatment outcome in depression: a meta-analysis. Am J Psychiatry 2012;169:141-151.

8. Kuo JR, Goldin PR, Werner K, Heimberg RG, Gross JJ. Childhood trauma and current psychological functioning in adults with social anxiety disorder. J Anx Dis 2011;25(4):467-473.

9. Varese F, Smeets F, Drukker M, et al. Childhood adversities increase the risk of psychosis: a meta-analysis of patient-control, prospective- and cross-sectional cohort studies. Schizophr Bull 2012;38(4):661-671.

10. Mueser KT, Rosenberg SD, Xie H, et al. A randomized controlled trial of cognitive-behavioral treatment for posttraumatic stress disorder in severe mental illness. J Consult Clin Psychol Apr 2008;76(2):259-271.

11. Frueh BC, Grubaugh AL, Cusack KJ, Kimble MO, Elhai JD, Knapp RG. Exposure-based cognitive-behavioral treatment of PTSD in adults with schizophrenia or schizoaffective disorder: a pilot study. J Anxiety Disord Jun 2009;23(5):665-675.

12. De Bont $P A$, van den Berg $D P$, van der Vleugel $B M$, et al. A multi-site single blind clinical study to compare the effects of prolonged exposure, eye movement desensitization and reprocessing and waiting list on patients with a current diagnosis of psychosis and co morbid post traumatic stress disorder: study protocol for the randomized controlled trial Treating Trauma in Psychosis. Trials 2013;14(151):doi: 10.1186/1745-6215-1114-1151. 
13. de Bont PA, van Minnen A, de Jongh A. Treating PTSD in patients with psychosis: a within-group controlled feasibility study examining the efficacy and safety of evidence-based PE and EMDR protocols. Beh ther Dec 2013;44(4):717-730.

14. De Graaf $R$, ten Have $M$, van Dorsselaer $\mathrm{S}$. The Netherlands Mental Health Survey and Incidence study-2 (NEMESIS-2): design and methods. Int J Meth Psychiat Res 2010;19(3):125141.

15. De Graaf R, Ormel J, Ten Have M, Burger H, Buist-Bouwman M. Mental disorders and service use in the Netherlands. Results from the European Study of the Epidemiology of Mental Disorders (ESEMeD). The WHO World Mental Health Surveys: Global Perspectives on the Epidemiology of Mental Disorders. Kessler, R.C., Üstün, T.B. ed: Cambridge University Press; 2008:388-405.

16. van Nierop M, van Os J, Gunther N, et al. Does Social Defeat mediate the association between childhood trauma and psychosis? Evidence from the Nemesis-2 study. Acta Psychiat Scand 2014;129(6):467-476.

17. van Dam $D$, van Nierop $M$, Viechtbauer W, Velthorst E, van Winkel R, GROUP Investigators. Childhood abuse and neglect in relation to presence and persistence of psychotic and depressive symptomatology. Psychol Med 2014; in press.
18. Heins $M$, Simons $C$, Lataster $T$, et al. Childhood trauma and psychosis: A case-control and case-sibling comparison across different levels of genetic liability, psychopathology, and type of trauma. Am J Psychiatry 2011;168(12):1286-1294.

19. Stewart AL, Hayes RD, Ware JE. The MOS short form general health survey. Medical Care 1988;26:724735.

20. Korver N, Quee PJ, Boos HB, Simons C, de Haan L. Genetic Risk and Outcome of Psychosis (GROUP), a multi site longitudinal cohort study focused on gene-environment interaction: objectives, sample characteristics, recruitment and assessment methods. Int J Meth Psychiat Res 2012;21(3):205-221.

21. Andreasen NC, Flaum M, Arndt S. The comprehensive assessment of symptoms and history (CASH). An instrument for assessing diagnosis and psychopathology. Arch Gen Psychiatry 1992;49(8):615-623.

22. Wing JK, Babor $T$, Brugha $T$, et al. SCAN. Schedules for Clinical Assessment in Neuropsychiatry. Arch Gen Psychiatry 1990;47(6):589-593.

23. Kay SR, Fiszbein A, Opler LA. The positive and negative syndrome scale (PANSS) for schizophrenia.

Schizophr Bull 1987;13(2):261-276. 
24. Bernstein DP, Stein JA, Newcomb MD, et al. Development and validation of a brief screening version of the Childhood Trauma Questionnaire. Child Abuse \& Neglect 2003;27:169-190.

25. Andrew $G$, Peters $L$. The psychometric properties of the Composite International Diagnostic Interview. Soc Psych Psych Epidem 1998;33:80-88.

26. The WHOQOL Group. Development of the World Health Organization WHOQOL-BREF quality of life assessment. Psychol Med 1998;28:551-558.

27. A.P.A. Diagnostic and Statistical Manual of Mental Disorders (DSM-IV$T R$ ), 4th edition. Washington, DC: American Psychiatric Association; 2000.

28. Statacorp. Stata Statistical Software:

Release 13. College Station, TX: StataCorp LP. 2013.

29. Conus $P$, Cotton $S$, Schimmelmann BG, Berk M, Daglas R, McGorry PD, Lambert M. Pretreatment and outcome correlates of past sexual and physical trauma in 118 bipolar I disorder patients with a first episode of psychotic mania. Bipolar Disord May 2010;12(3):244-252.

30. Boyette LL, van Dam D, Meijer C, et al. Personality Compensates for Impaired Quality of Life and Social Functioning in Patients With Psychotic Disorders Who Experienced Traumatic Events. Schizophr Bull Apr 252014.

31. van Nierop $M$, Lataster $T$, Smeets $F$, et al. Psychopathological mechanisms linking childhood traumatic experiences to risk of psychotic symptoms. Analysis of a large, representative population-based sample. Schizophr Bull 2014;40(suppl.2):S123-S130.

32. Ogino $S$, Lochhead $P$, Chan AT, et al. Molecular pathological epidemiology of epigenetics: emerging integrative science to analyze environment, host, and disease. Mod path : an official journal of the United States and Canadian Academy of Pathology, Inc Apr 2013;26(4):465-484.

33. Schumann G, Binder EB, Holte A, et al. Stratified medicine for mental disorders. European neuropsychopharmacology : the journal of the European College of Neuropsychopharmacology Jan 2014;24(1):5-50.

34. Owen DR, Rupprecht R, Nutt DJ. Stratified medicine in psychiatry: a worrying example or new opportunity in the treatment of anxiety? J psychopharm (Oxford, England) Feb 2013;27(2):119-122.

35. Teicher MH, Samson JA. Childhood maltreatment and psychopathology: A case for ecophenotypic variants as clinically and neurobiologically distinct subtypes. Am J Psychiatry Oct 1 2013;170(10):1114-1133.

36. van Winkel R. Etiological stratification as a conceptual framework for gene-by-environment interaction research in psychiatry. Epidem Psychiat Sciences 2014; In press.

37. Szyf M. The genome- and system-wide response of DNA methylation to early life adversity and its implication on mental health. Can j psychiatry Revue canadienne de psychiatrie Dec 2013;58(12):697-704. 
38. Perroud N, Dayer A, Piguet C, Nallet A, Favre S, Malafosse A, Aubry JM. Childhood maltreatment and methylation of the glucocorticoid receptor gene NR3C1 in bipolar disorder.

Br J Psychiatry Jan 2014;204(1):30-35.

39. Mehta D, Klengel T, Conneely KN, et al. Childhood maltreatment is associated with distinct genomic and epigenetic profiles in posttraumatic stress disorder. Proceedings of the National Academy of Sciences of the United States of America May 14 2013;110(20):8302-8307.

40. McGowan PO, Sasaki A, D'Alessio AC, Dymov S, Labonte B, Szyf M, Turecki $\mathrm{G}$, Meaney MJ. Epigenetic regulation of the glucocorticoid receptor in human brain associates with childhood abuse. Nat Neurosci Mar 2009;12(3):342-348.
41. de Arellano MA, Lyman DR, JobeShields L, et al. Trauma-focused cognitive-behavioral therapy for children and adolescents: assessing the evidence. Psychiatric services (Washington, DC) May 1 2014;65(5): 591-602.

42. Van Winkel R, Van Nierop M, MyinGermeys I, van Os J. Childhood Trauma as a Cause of Psychosis: Linking Genes, Psychology, and Biology. Can J Psychiatry 2013;58(1): 44-51.

43. Kelleher I, Keeley $\mathrm{H}$, Corcoran $\mathrm{P}$, et al. Childhood trauma and psychosis in a prospective cohort study: cause, effect, and directionality. Am J Psychiatry 2013;170(7):734-741. 
CHAPTER 12

Discussion 


\section{PSYCHOSIS}

\section{Measuring psychosis}

This thesis started with an investigation into the very basics of psychosis research; how psychosis is measured. In most studies, either a self-report measure or a clinical interview is used to establish positive psychotic experiences, usually depending on sample size. One important caveat in studies using self-report measures for psychosis is the fact that these yield high numbers of false-positives ${ }^{1}$. Thus, in these studies, a large amount of 'noise' may be incorporated in analyses, yielding unreliable results.

However, Bak and colleagues demonstrated that false-positive ratings of psychosis do carry important risk-related information, as these were strongly associated with future development of psychotic disorder ${ }^{2}$. Therefore, the characteristics of the group of individuals with false-positive ratings of psychosis were investigated in chapter two, in order to identify factors associated with the expression of mild psychotic experiences and how they can lead to need for care ${ }^{3}$. In this chapter, three groups were compared: (1) controls (individuals who did not self-report psychotic experiences; (2) the false-positive (FP) group (individuals who self-reported one or more psychotic experiences, none of which were confirmed by clinical interview); and (3) the true positive (TP) group (individuals who self-reported one or more psychotic experiences and for whom at least one psychotic experience was confirmed during clinical interview (see Figure 1).

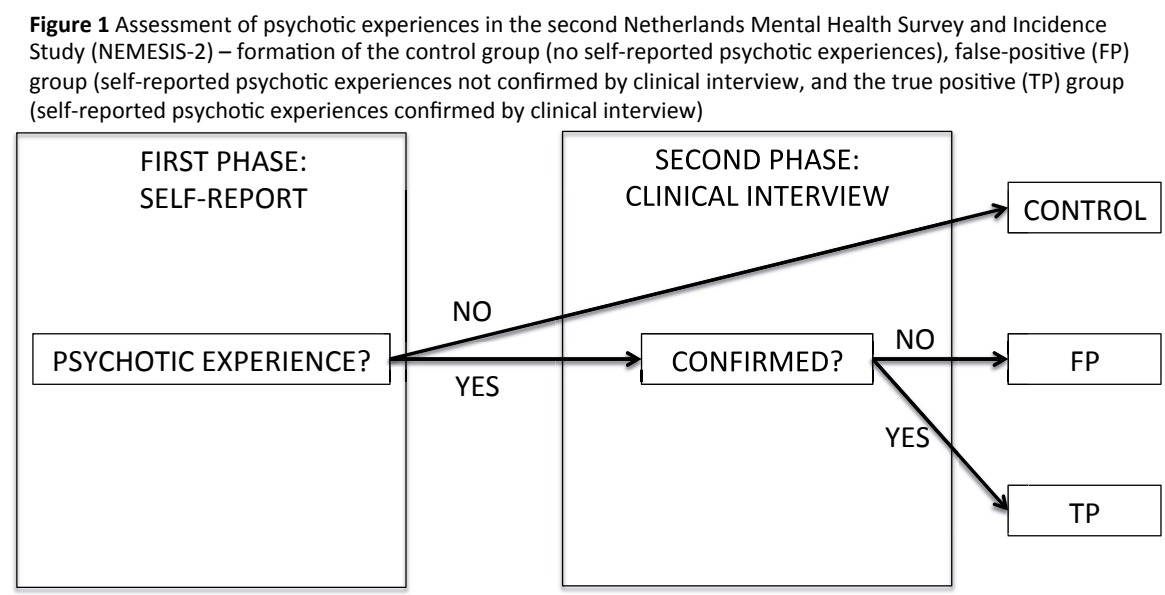

This study uncovered that the FP group, compared with the controls, were more often exposed to risk factors for psychosis, such as childhood trauma and cannabis use. They more often had mood, anxiety, or substance use disorders, and showed poorer social functioning. Thus, even though, based on the clinical interviews, these individuals were thought to have no psychotic experiences, they showed important psychosis-related characteristics. When comparing the FP group with the TP group, however, differences in terms of other psychopathology and risk factors were either non-significant or much smaller, with the exception of help-seeking behaviour and recency of the psychotic 
experiences. The TP group were more likely to have sought help for their psychotic experiences, and these experiences were more recent, which can be an important clue as to when clinicians assess an experience as psychotic. By contrasting these three groups, this study showed that FP psychotic experiences may represent the mildest expression of psychosis, and do not indicate absence of risk ${ }^{2}$. In line with Poulton and colleagues ${ }^{4}$, these results suggest that psychotic experiences may not be either true or false-positive, but index risk as a continuum reflecting the certainty with which reports are rated as psychotic.

\section{Transition to psychotic disorder}

Given the cross-sectional nature of the data used in chapter two, no inferences could be made about factors associated with transition to psychotic disorder. Therefore, using a longitudinal study, chapter three investigated which factors can predict future development of psychotic disorder ${ }^{5}$. Given the low prevalence of psychotic disorders such as schizophrenia, prospective studies assessing these risk factors in general population samples require very large samples. The classic yearly incidence rate for schizophrenia (percentage of the population that transitions to schizophrenia per year) is estimated at $0.02 \%$. Thus, for a 1 -year study, 5,000 individuals must be included to catch 1 individual who makes a transition to schizophrenia. As this is not very feasible, so-called high-risk studies are done for these types of investigations. In these studies, individuals at 'highrisk' for psychosis are included, and followed over a certain period of time. What constitutes as 'high-risk' can differ, but usually includes non-clinical psychotic experiences, and more importantly, help-seeking. This means that these individuals already display a relatively mild form of psychosis, and form a very specific group of individuals who are already at such a high level of concern about their mental state, that they are seeking professional help ${ }^{7,8}$. Transition to psychosis within this population therefore does not refer to transition from health to psychosis, and thus any found associations with risk factors are not based on true transitions.

In chapter three, another approach to this investigation was implemented. A large sample of healthy (non-psychotic) controls $(n=462)$ and unaffected siblings of patients with a psychotic disorder $(n=810)$ were followed over a period of three years. By including these two samples, not only the environmental risk could be assessed, but also the possible influence of genetic risk could be included in the analyses (as the siblings are at elevated genetic risk for psychosis). The exposures assessed in this study were minority position ${ }^{9}$, urban birth ${ }^{10}$, cannabis use ${ }^{11}$, and childhood trauma ${ }^{12}$. Within this sample, it was found that exposure rates were very high; $68 \%$ of the siblings, and $60 \%$ of the controls, were exposed to at least one environmental factor. In total, 11 individuals made a transition to psychotic disorder. All of these individuals were exposed to at least one environmental factor, whereas $65 \%$ of the non-transition individuals were exposed to an environmental factor.

By comparing these estimates, combining them with estimates of (proxy) genetic risk, the proportion of transitions in the population attributable to environmental or genetic risk (PAF) could be calculated, which ranged from $28 \%$ for minority status, $45 \%$ for urban birth, 
$57 \%$ for cannabis use, $86 \%$ for childhood trauma, and $50 \%$ for high-risk sibling status. All transitions were associated with environmental exposure, with the greatest effect for childhood trauma. This study provided further evidence for a causal association between environmental exposure and psychosis, as no evidence was found for gene-environment correlation.

This chapter discussed the similarity of the finding of high rates of environmental exposures compared with current considerations in genetics research; the risk of schizophrenia is thought to be dependent on thousands of common alleles of very small effect $^{13}$, and therefore the genetic risk to psychosis is present in the entire population, to a lesser or greater degree. Analogously, most individuals in the current sample are exposed to environmental risk factors, yet only some of them transition to psychosis. As these environmental risk factors are so common, the impact of these factors can only be detected against the background of differential genetic risk, indicating gene-environment interaction.

\section{Mechanisms of psychosis}

In chapters four through seven, possible underlying mechanisms linking childhood trauma to psychosis were investigated. In chapter four, five existing psychological models were examined using a large representative sample of the Dutch population $(n=13,722)$, and utilizing the most appropriate statistical methods ${ }^{14}$.

Contrary to previous findings ${ }^{15,16}$, no evidence was found that childhood trauma shows a stronger association with delusions than with hallucinations, nor that particular types of trauma show a stronger association with auditory-verbal hallucinations (AVH), or with paranoia. Furthermore, while other researchers found that abuse was more likely to lead to psychosis than neglect ${ }^{17,18}$, no such specificity was found in this chapter.

The current study did replicate earlier findings ${ }^{19}$ of a greater likelihood of co-occurring hallucinations and delusions in traumatized individuals, compared with presence of either symptom in isolation. These findings, combined with earlier work $^{19}{ }^{20}$, suggest that childhood trauma increases risk for intensification of the psychosis process (delusion formation compounding existing hallucinatory experiences), causing build-up to more complex psychotic states that can increase risk for help seeking and clinical decompensation. Moreover, the experience of "intention-to-harm" (e.g. abusive events) was more likely to have a psychotic outcome than trauma with a non-intentional nature (e.g. the death of a loved one). In line with earlier work ${ }^{21}$, this could indicate that perceived social threat could be the key risk factor for psychosis. Alternatively, childhood traumatic experiences, in particular with an intentional nature, could induce a state of social defeat, or a perceived 'outsider status', which in turn increases risk for psychosis ${ }^{22}$.

This last theory is reviewed in more detail in chapter five ${ }^{23}$. The social defeat hypothesis of psychosis was originally postulated in order to link together several known risk factors for 
psychosis; growing up in an urban environment, hearing impairment, low IQ, minority status, and childhood trauma ${ }^{22}$. The higher levels of competition in urban environments, social exclusion associated with minority status or hearing impairment, fewer job opportunities for individuals with a lower IQ, and humiliation associated with childhood abuse may all induce a state of social defeat. Even though this theory was only directly tested and corroborated in one human study ${ }^{24}$, a large amount of animal studies have shown that specific social defeat stress, induced by repeated attacks by a stronger intruder animal, leads to long-term abnormalities in dopaminergic neurotransmission ${ }^{25}, 26$. As dopamine transmission plays an important role in psychotic symptoms ${ }^{27}$, the viability of this link between psychology and biology in humans seems plausible.

The one human study linking a state of social defeat to psychosis mentioned above is based on a relatively small sample of individuals at ultra high risk for psychosis ${ }^{24}$. Therefore, in chapter six, the social defeat hypothesis is investigated using a large representative general population sample ${ }^{28}$. The state of social defeat was defined as feelings of selfdevaluation, such as being hopeless about the future, and having a loss of self-confidence. By utilizing specific mediation analyses, it was found that individuals exposed to trauma were (i) more likely to report a state of social defeat, (ii) were also more likely to report psychotic symptoms, and, most importantly, (iii) that the link between childhood trauma and psychosis was mediated by social defeat.

Even though this body of work certainly increases the plausibility of a causal association between childhood trauma and psychosis, gene-environment correlation may still explain a large proportion, if not all, of the found association ${ }^{23}$. As it has proven very difficult to replicate genetic factors associated with psychosis by using candidate gene approaches ${ }^{29}$, chapter seven ${ }^{30}$ utilizes polygenic risk scores based on the most recent genome-wide association study (GWAS) of schizophrenia ${ }^{31}$. In this chapter, the association between polygenic risk score and psychosis was replicated. More importantly, reports of childhood trauma were not associated with polygenic risk. Moreover, the association between childhood trauma and psychosis remained similar after adding polygenic risk score as a covariate. These last two findings indicate that individuals with higher polygenic risk, which currently is our strongest indicator for genetic vulnerability for psychosis, are not more likely to experience childhood trauma, and that the association between trauma and psychosis remains after controlling for genetic risk. These findings argue against geneenvironment correlation explaining most of the link between trauma and psychosis, adding to other studies reporting similar evidence ${ }^{32}$. 


\section{OUTCOME OF CHILDHOOD TRAUMA}

\section{Differential impact of childhood trauma}

Four main topics were discussed in chapter eight: (i) the differential impact of trauma on positive and negative psychotic symptoms, (ii) the influence of trauma on the course of psychotic symptoms, (iii) the difference in impact of abuse or neglect on symptoms, and (iv) the differential impact of trauma on psychotic or affective symptoms ${ }^{33}$. The sample for this study consisted of psychotic disorder patients, their unaffected siblings, and controls. An earlier study found that abuse is more likely to induce positive psychotic symptoms compared with negative symptoms, whereas neglect shows a comparable impact on positive and negative symptoms ${ }^{18}$. These findings were confirmed in the current study, using a larger sample and more sophisticated statistical techniques. This study was the first to assess whether trauma is related to a differential course of symptoms (i.e. whether symptoms in traumatized individuals increase or decrease to a similar extend compared with non-traumatized individuals). No such differential course was found; psychotic symptom severity decreased to a similar extent in both groups. When comparing the impact of abuse to that of neglect, some between-group differences were found; in the siblings and controls, abuse and neglect had a similar impact on psychotic symptoms, whereas in the patients abuse showed a stronger association with psychosis than neglect. This last finding is noteworthy, given the finding of chapter four (no difference in impact of abuse versus neglect). In that study, however, a general population sample was used, which arguably is more similar to the siblings and controls than to the group of psychotic disorder patients. However, it may be possible that the patient group was exposed to higher levels of abuse severity, compared with the siblings or controls, or the general population. Thus, the impact of abuse may be more stressful than that of neglect, however, only when the severity of abuse reaches a certain threshold, impact on the stress-response system becomes most pronounced ${ }^{34}$, and a differential psychotic outcome compared with neglect can be observed.

The last question within this study examined the differential impact of trauma on psychotic and affective symptoms. Especially within the patient and sibling groups, individuals at above-average genetic risk for psychosis, one could assume that the association between trauma and psychotic symptoms would be stronger than with affective symptoms. Surprisingly, the current study uncovered that either the impact of trauma on psychosis and depression was similar, or the association with depression was stronger. These findings indicate that childhood trauma may contribute to a shared vulnerability for psychosis and depression, possibly related to a dysregulation of the stress-response system, which has been shown to be associated with both disorders ${ }^{20,35}$.

This raises another important question; at least in the patient group, there is a presence of co-occurrence of symptoms, across diagnostic boundaries (patients with a psychotic disorder, who also develop symptoms of depression). The impact of trauma on this co-occurrence, and on disease severity, warrants further investigation. 


\section{Co-occurrence of symptoms}

In chapter nine, instead of focusing on patients with a psychotic disorder, the impact of (subclinical) psychotic symptoms on disease severity in patients with a mood or anxiety disorder was investigated ${ }^{36}$. Even though individuals with an affective disorder often report (subclinical) psychotic symptoms ${ }^{37,38}$, these symptoms are usually neglected unless they are dominant and clinically severe. However, co-occurrence of psychotic symptoms in non-psychotic disorders predicts poorer outcome ${ }^{39,40}$, and affective and psychosis symptoms may impact on each other ${ }^{41-43}$. Furthermore, affective and psychotic pathology share similar environmental risk factors, and shared genetic liabilities have been suggested ${ }^{44-46}$. Therefore, chapter nine investigated the differences between individuals with an anxiety or depressive disorder with or without psychotic symptoms.

Firstly, evidence was found that psychotic symptoms were not rare in these individuals: nearly one third reported (subclinical) hallucinations and/or delusions. Individuals with psychotic symptoms displayed an increased illness severity, poorer illness course, and more help seeking behaviour, than individuals without psychotic symptoms. These findings indicate that co-occurrence of symptoms not only is not rare, but also very clinically relevant. Importantly, subjects reporting co-occurring psychotic symptoms also had a higher likelihood of a childhood trauma history, indicating a higher etiological loading in these individuals. The progression from affective symptoms to psychotic symptoms has been described before, resulting in a hypothesis called 'the affective pathway to psychosis'. This theory postulated that first affective symptoms develop (possibly induced by exposure to trauma), which, when these become severe enough, give rise to psychotic symptoms ${ }^{47}$. In essence this hypothesis describes the development of psychosis as an end-product of severe affective dysregulation.

Based on the findings of chapter nine, chapter ten investigated the link between trauma and co-occurring symptoms in more detail ${ }^{48}$. Within this study, a representative general population sample was used, as well as a sample of patients with a psychotic disorder, patients with a lifetime mood disorder, and patients with a lifetime anxiety disorder. Across all these samples, childhood trauma exposed individuals were more likely to report co-occurrence of psychotic, anxious and affective symptoms, rather than any of these symptoms in isolation. These findings could be evidence for the affective pathway to psychosis $^{47}$; as affective symptoms become more pronounced, co-occurring psychotic symptoms may have developed. In order to address this alternative hypothesis, additional analyses were performed. These analyses indicated that trauma was associated with co-occurring symptoms in individuals without a psychiatric disorder, and who had never sought help for their subclinical symptoms. Moreover, the association between trauma and co-occurring symptoms remained strong and significant after controlling for symptom severity load. Thus, psychotic symptoms co-occurred with other symptoms before any severe affective dysregulation had developed, and this co-occurrence was independent of symptom severity. These findings indicate that psychotic symptoms are not the endproduct of severe affective dysregulation, but rather an integral part of psychopathology following childhood trauma. 
The fact that this co-occurrence (or clustering) of symptoms in traumatized patient groups was present, already presents us with some important clinical relevance. However, additional detailed insights in functional outcome are needed to get a greater understanding of the exact and practical meaning of these findings. Therefore, in chapter eleven, the three patient groups of chapter ten were investigated in more detail (psychotic, anxiety, and depressive disorder patients) ${ }^{49}$. Each patient group was divided according to childhood trauma history, and clustering of symptoms, resulting in four subgroups: (1) patients without a trauma history, and without clustering of symptoms (CT-/CL-); (2) patients without a trauma history, and with clustering of symptoms (CT-/CL+); (3) patients with a trauma history, and without clustering of symptoms ( $C T+/ C L-)$; and (4) patients with a trauma history, and with clustering of symptoms (CT+/CL+) (see Figure 2).

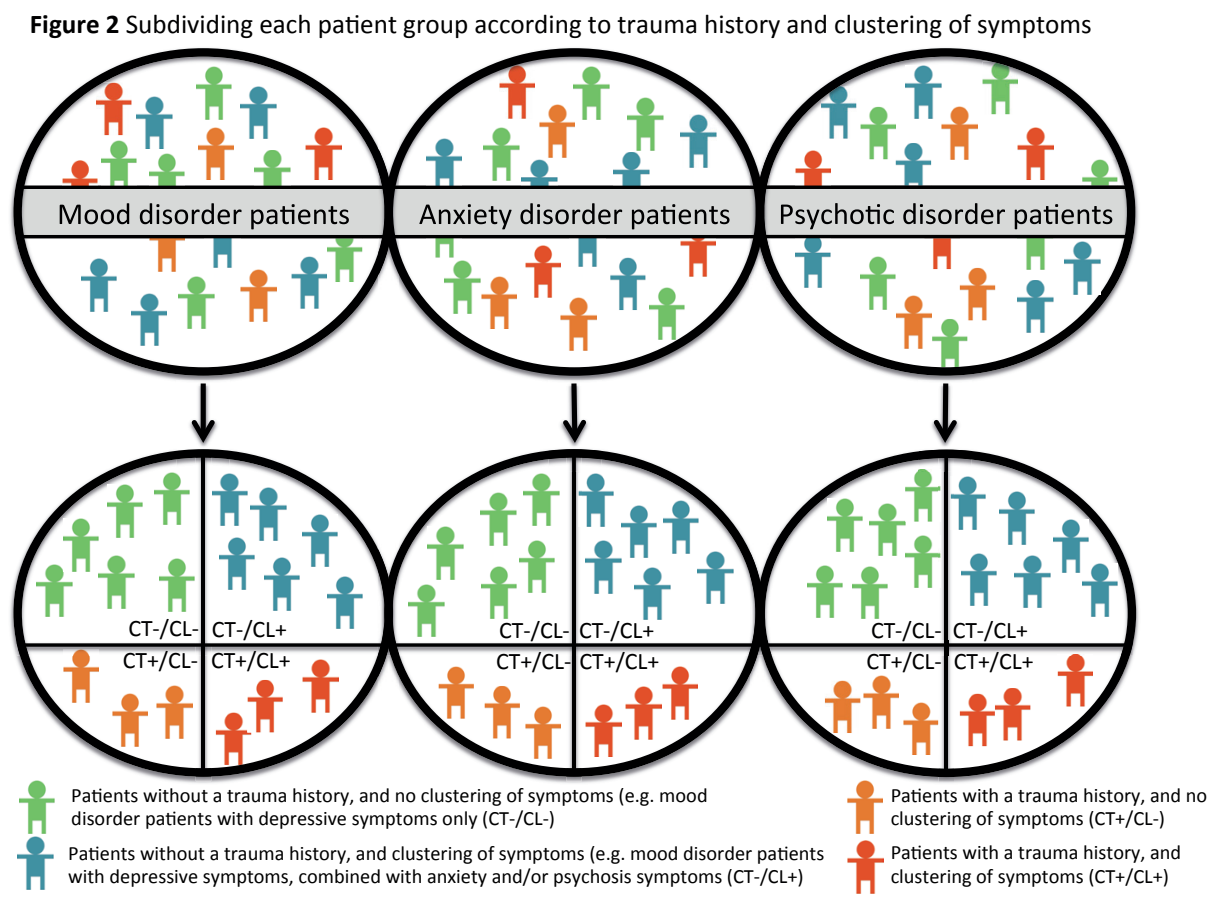

Subsequently, differences in functional outcome between these four groups were investigated. Some examples of these functional variables were: disease severity, help seeking behaviour, social functioning, occupational functioning, comorbid drug or alcohol disorders, and quality of life. In all three patient groups, traumatized individuals with clustering of symptoms displayed a poorer functional outcome, when compared to individuals without a trauma history, without clustering of symptoms, or neither. These findings show that the trauma-related admixture of symptoms is associated with clinically highly meaningful differences in functional outcome. 


\section{Childhood trauma as stratifying factor}

Why are the findings of co-occurrence or clustering of symptoms in traumatized individuals important? The relevance of co-occurring symptoms is clear; it predicts poorer illness outcome, and increased disease severity. Yet, this knowledge does not help us a great deal to provide better care for patients. The finding that childhood trauma specifically is associated with this co-occurrence might help us develop better care, however. In order to clarify that, first some important differences between somatic and psychiatric medicine should be discussed.

When someone presents with a somatic complaint (e.g. a lump in their breast), physicians have a wide array of clinical tests available to investigate which disease this patient might have. They can palpate this lump, do a scan to see what it looks like, and they can do a biopsy. All this information together can aid the physician to make an unambiguous diagnosis of for example a benign cyst, or breast cancer. Even though exceptions certainly exist, most somatic diseases can be diagnosed in a similar fashion, using clinical tests that are based on the biological features of somatic diseases. These somatic diagnostic categories have changed as medical science progressed, but they are preferably based on underlying biological mechanisms.

In psychiatric medicine, classification of disorders is based on diagnostic categories as well. For example, a patient may receive a diagnosis of schizophrenia, or major depressive disorder. These classifications, however, are not based on their biological features, but stem from observed behaviour and patients' reports of feelings. Nonetheless, in the past few decades, major efforts have been made to develop clinical tests that can distinguish between these classifications. For example, Magnetic Resonance Imaging (MRI) studies have been done to see whether there are structural or functional differences between the brains of patients with schizophrenia, and those of healthy controls ${ }^{50}$. Or blood tests were developed that could help diagnose depression and predict drug response, such as the Dexamethasone Suppression Test ${ }^{51, a}$. However, either findings from such studies were not replicated, provided very low accuracy, or were only useful when comparing textbook cases to super healthy controls, a situation in which no clinician would actually need an additional test for confirmation ${ }^{52}$. The major issue preventing us from finding useful clinical tests for psychiatric medicine is therefore based on a faulty premise; it is very unlikely that we'll find a biological test for a diagnostic classification that is not based on biological features.

Within somatic medicine, another very useful tool is being used to aid the diagnostic process and increase prognostic accuracy: stratified medicine. Going back to the example of the patient with a breast lump, a family history of breast cancer can be established. Given our knowledge of the genetics of breast cancer, a patient with high familial loading will be more likely to develop breast cancer as well ${ }^{53}$.

\footnotetext{
${ }^{a}$ In the Dexamethasone Suppression Test, cortisol levels in the blood are measured after administering dexamethasone. It was thought that depressive patients have higher cortisol levels in response to this injection, compared with healthy controls.
} 
Or assessment of smoking habits and alcohol use, a form of environmental stratification, can help predict the outcome in patients with throat cancer ${ }^{54}$. Stratified medicine is not only useful when applied within certain diagnostic categories, but has proven to be particularly helpful across diagnostic boundaries, the most famous example for which originated in breast cancer research. Overexpression of human epidermal growth factor subtype 2 (HER2) was found to be associated with a particularly poor prognosis in breast cancer ${ }^{55}$. Using a stratified approach, HER2 was also found to be associated with a poor prognosis in other types of cancer, such as ovarian, lung, or gastric cancer ${ }^{56}$. Now, specific treatment approaches for these subtypes of cancer are being implemented, increasing survival odds in all these cancer types ${ }^{56}$. Turning back to the findings of this thesis, one

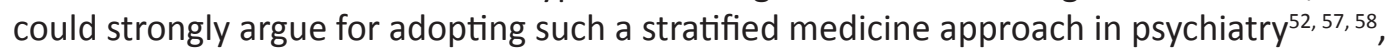
using childhood trauma history as an environmental stratifying factor. By dividing one patient group (i.e. a group of patients all sharing the same diagnosis) according to their trauma history, a specific phenotype of an admixture of psychopathology was found (see Figure 3). The substantial subgroup of patients exposed to trauma, and in particular patients exposed to trauma with an admixture of psychopathology, displayed a poor functional outcome. Moreover, these findings translated across diagnostic categories, as similar phenotypic and functional patterns were found in several patient groups (Figure 3).

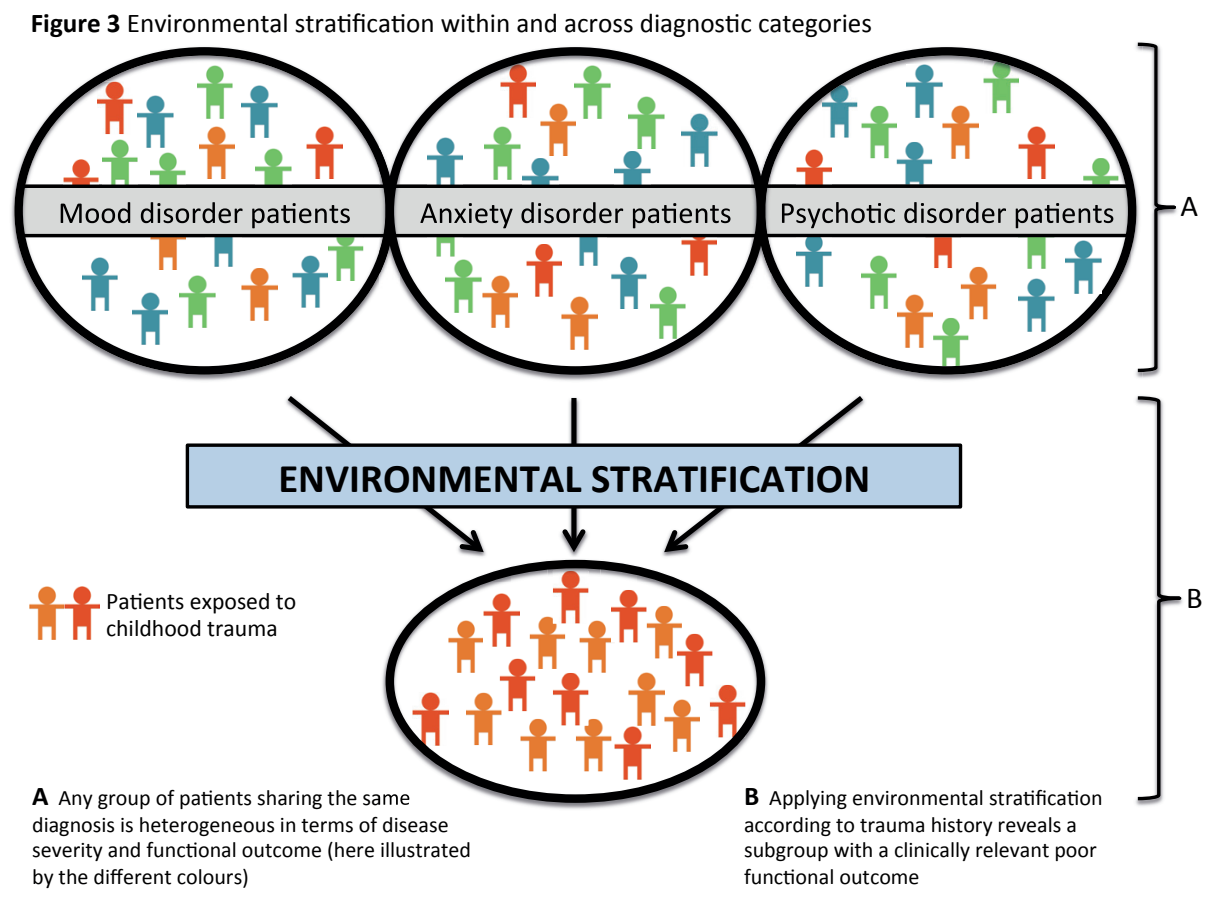

These findings echo those discussed in a recent review, in which the similar patterns in terms of earlier disease onset, greater clinical severity, lower response to treatment, and similar brain abnormalities in traumatized individuals across several mental disorders such as depression and anxiety disorders are highlighted ${ }^{59}$.

One viable underlying mechanism that may explain these similarities across mental 
disorders can be found in the field of epigenetics ${ }^{60}$. Epigenetics is the mechanism that allows us to adapt to our environment. Basic genetics operate by DNA providing instructions to RNA that in turn forms protein structures based on these instructions (i.e. transcription). These protein structures are the very basis of all life, and are needed for assembly, maintenance, and reproduction. Epigenetics, on the other hand, are subject to external influence, such as stress ${ }^{61}$. This external influence sets in motion epigenetic processes, which are responsible for which genes will, or will not, come to expression (thus which genes will be 'turned on' or 'turned off'). There are many forms of epigenetic functioning, but the main components are DNA methylation, and histone tail modification (Figure 4). In DNA methylation, methyl binding proteins bind to DNA, which disrupts transcription, and results in 'gene-silencing' (i.e. processes normally initiated by that gene stop, see Figure 4). Histone tail modification can either drive transcription (i.e. processes from that gene are initiated) or repress transcription, depending on structural changes of the DNA molecule. Epigenetic processes can change the structure of the DNA molecule to a heterochromatin, where the structure is condensed, or a euchromatin, which has an open structure. In a heterochromatin, histone proteins are tightly packed, which blocks access of transcription factors (Figure 4). In a euchromatin, the open structure allows access for transcription factors, which drives transcription (Figure 4).

Figure 4 The main components of the epigenetic code

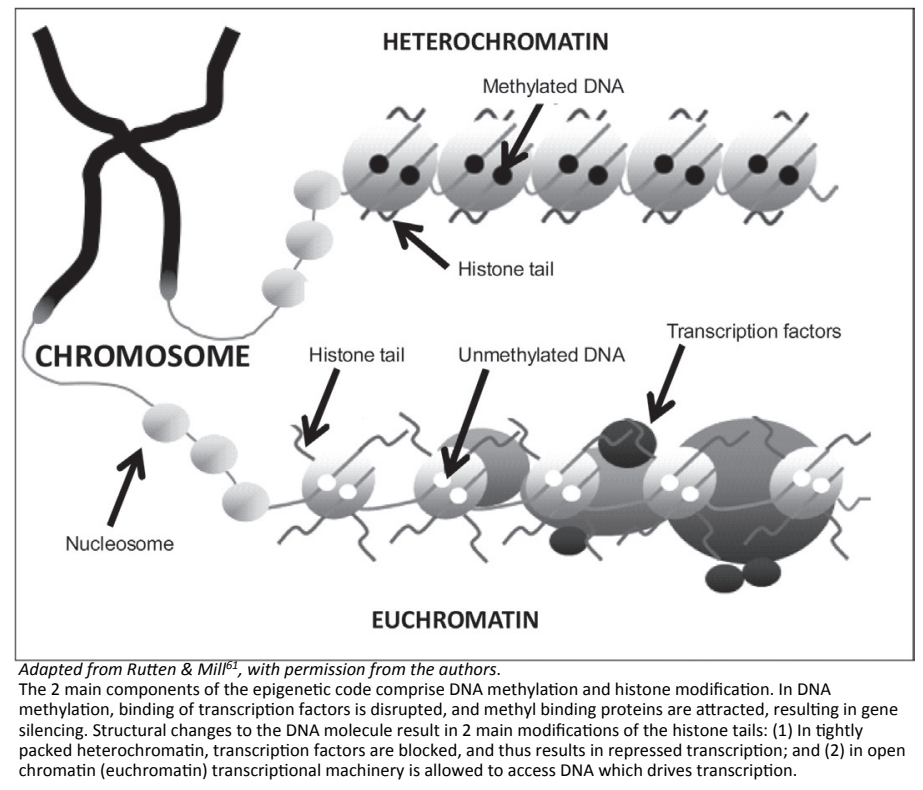

One important function of epigenetic mechanisms is that of cell differentiation. Our DNA is identical in each stem cell, yet each stem cell can transform into any cell, such as a skin cell, or brain cell. Epigenetic mechanisms, influenced by the outside environment, make sure that the right part of the DNA is expressed or transcribed, and a stem cell can differentiate into the right type of cell. Epigenetic mechanisms can, however, also have a detrimental outcome. Particularly environmental exposures, occurring in a critical period 
in development, such as childhood trauma, can result in system wide altered DNA methylation patterns, leading to somatic and mental health problems later in life ${ }^{60}$.

Stratification according to trauma history is already widely applied in epigenetic studies. For example, when comparing post-traumatic stress disorder (PTSD) patients with a childhood trauma history with PTSD patients without a childhood trauma history, Mehta and colleagues found almost completely non-overlapping transcription profiles (i.e. each group showed highly distinct biological modifications, dependent on childhood trauma history $)^{62}$. Similarly, in two studies investigating bipolar disorder patients, and suicide victims, respectively, higher levels of DNA methylation were found, depending on abusive experiences ${ }^{63}$, their frequency ${ }^{64}$, and severity ${ }^{64}$. Each of these last two studies found these methylation patterns in a gene known to be partly responsible for our biological stress response system, or hypothalamic-pituitary-adrenal (HPA) axis ${ }^{65}$. Therefore, childhood traumatic experiences have very likely set in motion epigenetic processes, which directly altered the way individuals from these samples were able to handle stress.

\section{Unique disease principle}

These findings do not necessarily negate the validity of the current classification system for psychiatric disorders. For many patients, treatments based on current diagnostic categories are very helpful, and getting a certain diagnosis will help them understand their disease better, as they will most likely share many features or symptoms with other patients with the same diagnosis. Nonetheless, a large amount of heterogeneity exists within patient groups, be it in symptom profiles, or in functional outcome. One patient receiving a diagnosis of schizophrenia may respond very well to treatment, or will need relatively low levels of intervention, or even none at all, maintain a fruitful occupational status, meaningful social relationships, and will in general enjoy life. Yet another patient, receiving the exact same diagnosis, can need lifelong high doses of antipsychotics with all bothersome side effects that go with it, will be unable to work or find some other constructive activity, will not be able to maintain relationships with friends or family, and think that their life is meaningless. A substantial part of this heterogeneity can be accounted for by stratifying according to childhood trauma history, but certainly not all heterogeneity is explained.

An explanation for this may be provided by the 'unique disease principle'66. As most of us (excluding monozygotic twins) have a unique DNA profile, and therefore a unique epigenome, and all of us have unique environmental exposures, possible outcomes of interactions between the epigenome and exposures are endless. The way psychiatric classification works now is essentially a Gestalt-type approach; as long as the general overall pattern of symptoms is recognizable as a diagnostic category, the patient will receive this diagnosis, much like how our brain will recognize and classify 'dog' when we see a Chihuahua or a Saint Bernard. The 'unique-disease-principle' provides an alternative to classic epidemiology or psychiatric classification, by stating that each individual can develop a unique outcome or disease based on our epigenome and experiences. Environmental 
stratification can provide us with a practical bridge between a possibly insufficient psychiatric diagnostic system and an unpractical unique disease principle (Figure 5).

Figure 5 Environmental stratification and the 'Unique Disease Principle'
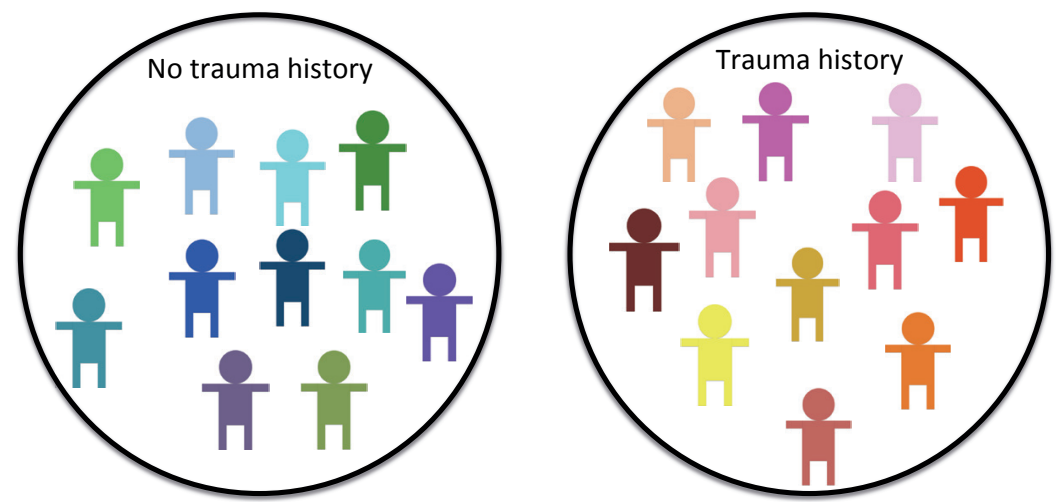

According to the 'unique disease principle', each individual can develop a unique outcome or disease, based on unique epigenomes and environmental exposures (here indicated by the unique colours). A substantial part of the heterogeneity in disease severity or functional outcome is accounted for by environmental stratification, according to childhood trauma history.

\section{Future directions}

Given the poor functional outcome, the subgroup of patients with a trauma-related admixed phenotype may be more resistant to treatment. This provides us with important avenues for additional targeted treatment options, by providing treatment plans specifically aimed at diminishing the detrimental effects of traumatic experiences. Currently, these treatment plans are mainly offered to patients with overt post-traumatic stress symptoms. Findings from this thesis would argue that offering these treatment plans to other patients as well, such as trauma-focused cognitive behavioural therapy or eye movement desensitization (EMDR), could prove very beneficial outside the context of PTSD or other trauma related anxiety disorders. Further studies are urgently needed to assess effectiveness of these treatment plans in individuals with trauma-related mixed phenotypes. One study, recently initiated, is investigating the safety and efficacy of prolonged exposure (PE) and EMDR therapies in psychotic disorder patients with comorbid PTSD $^{67}$, with promising results ${ }^{68}$.

But before clinical practice can even move forward to such an approach, another hurdle needs to be addressed: when patients present with mental problems (but not posttraumatic stress symptoms), and do not spontaneously report traumatic events from their childhood, health care professionals tend to not ask about childhood trauma ${ }^{69}$. This (almost) taboo is maintained from both sides. Patients may not really want to talk about difficult events from their past, or are less aware that they could still have a profound effect in adulthood. Health care professionals, on the other hand, may assume no such past exists as their patients haven't mentioned it, or even think that digging up events from long ago 
may exacerbate symptoms, especially in psychotic disorder patients ${ }^{69}$. Findings from this thesis, combined with other reports, however, would argue that assessing trauma history in all patients first presenting with mental problems may prove very beneficial for both patients and health care professionals.

The studies from this thesis utilized data from adult samples. Longitudinally investigating symptom patterns and functional outcome in response to childhood trauma in samples of children studied may provide important evidence for a causal association between trauma and clustering of symptoms. Studies utilizing such a design, including careful assessment of psychopathological profiles and personality styles, may provide insights into the chronological order of exposure and outcome, and developmental trajectories of psychopathology. Additionally, replicating these findings in such samples could provide compelling evidence demanding more efforts for primary prevention, and important avenues for secondary prevention. Arguably any trauma-related treatment may be much more effective when applied immediately, rather than decades later.

\section{Developmental pathway}

Combining the findings of this thesis provides a possible developmental pathway to psychopathology and functional outcome (Figure 6). Individuals exposed to childhood trauma, in particular trauma with an intentional nature (i.e. abuse), may develop a mixed phenotype of psychopathology, consisting of co-expression of symptoms of psychosis (in particular co-occurring hallucinations and delusions), anxiety, mania, and depression. These symptoms of depression are in particular characterized by feelings fitting a state of social defeat (e.g. feelings of hopelessness, loss of self-confidence). In chapter 6 , social defeat was initially described as a mediator between childhood trauma and psychosis. However, the symptoms of psychosis and the expression of social defeat may have co-occurred, and following the findings of chapter 10 , this state of social defeat is most likely part of the overall mixed phenotype of psychopathology, rather than a mediator. The symptom clusters within this mixed phenotype may still impact on each other; detailed temporal analyses may uncover the specifics of such a mechanism. Ultimately, especially those individuals who are exposed to trauma, and have this mixed phenotype of psychopathology, have a poor functional outcome, in terms of social functioning, clinical severity, and quality of life. Intervening on this developmental pathway may come in the form of primary or secondary prevention, or trauma-related therapies in adulthood. 
Figure 6 Developmental pathway of childhood trauma, to psychopathology and functional outcome

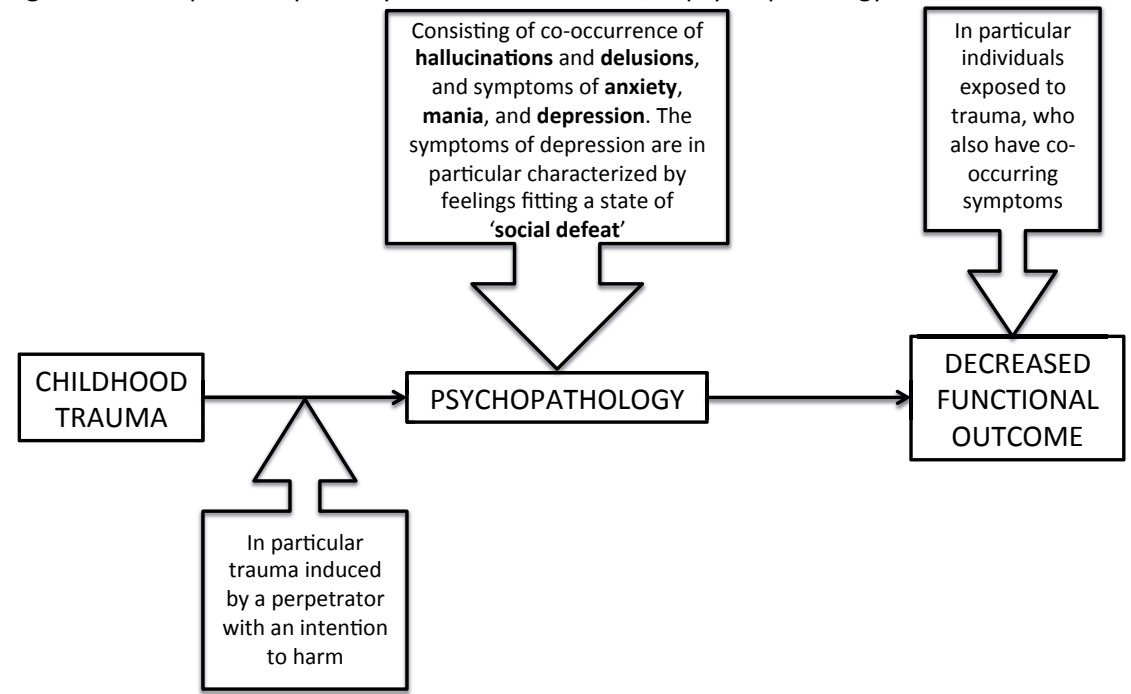

Childhood traumatic experiences, in particular those with an intentional nature, may lead to psychopathology in adulthood. This psychopathology consists of co-occurring hallucinations and delusions, and symptoms of anxiety, mania, and depression, the latter characterized by feelings of hopelessness, and loss of selfconfidence (i.e. a state of social defeat). Particularly those individuals exposed to trauma, with co-occurring symptoms, display a decreased functional outcome. 


\section{REFERENCES}

1. Kelleher I, Harley M, Murtagh A, Cannon M. Are screening instruments valid for psychotic-like experiences? A validation study of screening questions for psychotic-like experiences using in-depth clinical interview. Schizophr Bull 2009;37(2):362-369.

2. Bak M, Delespaul P, Hanssen $M$, de Graaf R, Vollebergh W, van Os J. How false are "false" positive psychotic symptoms? Schizophr Res 2003;62:187-189.

3. van Nierop M, Van Os J, Gunther N, et al. Phenotypically continuous with clinical psychosis, discontinuous in need for care: Evidence for an extended psychosis phenotype. Schizophr Bull 2012;38(2):231-238.

4. Poulton R, Caspi A, Moffitt TE, Cannon M, Murray R, Harrington H. Children's self-reported psychotic symptoms and adult schizophreniform disorder: A 15 year longitudinal study. Arch Gen Psychiatry 2000;57:1053-1058.

5. van Nierop M, Janssens M, GROUP. Evidence that transition from health to psychotic disorder can be traced to semi-ubiquitous environmental effects operating against background genetic risk. Plos One 2013;8(11):e76690.

6. Sutterland AL, Dieleman J, Storosum $J G$, et al. Annual incidence rate of schizophrenia and schizophrenia spectrum disorders in a longitudinal population-based cohort study. Soc Psych Psych Epidemiol Sep 2013;48(9):1357-1365.

7. Fusar-Poli $P$, Nelson $B$, Valmaggia $L$, Yung AR, McGuire PK. Comorbid Depressive and Anxiety Disorders in 509 Individuals With an At-Risk Mental State: Impact on
Psychopathology and Transition to Psychosis. Schizophr Bull 2012; doi:10.1093/schbul/sbs136.

8. Fusar-Poli P, van Os J. Lost in transition: setting the psychosis threshold in prodromal research. Acta Psychiat Scand 2013;127(3):248-252.

9. Cantor-Graae E, Selten JP. Schizophrenia and migration: a meta-analysis and review. Am J Psychiat 2005;162:12-24.

10. Vassos E, Pedersen CB, Murray R, Collier DA, Lewis CM. Meta-analysis of the association of urbanicity with schizophrenia. Schizophr Bull 2012;38(6):1118-1123.

11. Matheson SL, Shepherd AM, Laurens $\mathrm{KR}$, Carr VJ. A systematic meta-review grading the evidence for non-genetic risk factors and putative antecedents of schizophrenia. Schizophr Res Dec 2011;133(1-3):133-142.

12. Varese F, Smeets F, Drukker M, et al. Childhood adversities increase the risk of psychosis: a meta-analysis of patient-control, prospective- and cross-sectional cohort studies. Schizophr Bulletin 2012;38(4):661-671.

13. Purcell SM, Wray NR, Stone JL, Visscher PM, O'Donovan MC, Sullivan PF, Sklar P. Common polygenic variation contributes to risk of schizophrenia and bipolar disorder. Nature 2009;460(7256):748-752.

14. van Nierop $M$, Lataster $T$, Smeets $F$, et al. Psychopathological mechanisms linking childhood traumatic experiences to risk of psychotic symptoms. Analysis of a large, representative population-based sample. Schizophr Bull 2014;40(suppl.2):S123-S130. 
15. Read J, Agar K, Argyle N, Aderhold V. Sexual and physical abuse during childhood and adulthood as predictors of hallucinations, delusions and thought disorder. Psychol Psychother: Theo Res Pract 2003;76:1-22.

16. Bentall R, Wickham $S$, Shevlin $M$, Varese F. Do specific early-life adversities lead to specific symptoms of psychosis? A study from the 2007 the adult psychiatric morbidity survey. Schizophr Bull 2012;38(4):734-740.

17. Shevlin M, Dorahy M, Adamson G. Childhood traumas and hallucinations: An analysis of the National Comorbidity Survey. J Psychiatr Res 2007; 41:222-228.

18. Heins $M$, Simons $C$, Lataster $T$, et al. Childhood trauma and psychosis: A case-control and case-sibling comparison across different levels of genetic liability, psychopathology, and type of trauma. Am J Psychiat 2011;168(12):1286-1294.

19. Smeets F, Lataster T, Dominguez MG, Hommes J, Lieb R, Wittchen HU, van Os J. Evidence That Onset of Psychosis in the Population Reflects Early Hallucinatory Experiences That Through Environmental Risks and Affective Dysregulation Become Complicated by Delusions. Schizophr Bull 2012;38(3):531-542.

20. Kapur S. Psychosis as a state of aberrant salience: a framework linking biology, phenomenology, and pharmacology in schizophrenia. Am J Psychiat 2003;160:13-23.

21. Arseneault $L$, Cannon $M$, Fisher $H$, Polanczyk G, Moffitt TE, Caspi A. Childhood trauma and children's emerging psychotic symptoms: a genetically sensitive longitudinal cohort study. Am J Psychiat 2011;168:65-72.

22. Selten JP, Cantor-Graae E. Hypothesis: social defeat is a risk factor for schizophrenia? B J Psychiat 2007;191(suppl. 51):s9-s12.

23. Van Winkel $R$, Van Nierop $M$, MyinGermeys I, van Os J. Childhood Trauma as a Cause of Psychosis: Linking Genes, Psychology, and Biology. Can J Psychiat 2013;58(1):44-51.

24. Stowkowy J, Addington J. Maladaptive schemas as a mediator between social defeat and positive symptoms in young people at clinical high risk for psychosis. Early Interv Psychiat 2012;6:87-90.

25. Krishnan V, Han MH, Graham DL, et al. Molecular adaptations underlying susceptibility and resistance to social defeat in brain reward regions. Cell 2007;131:391-404.

26. Morgan $D$, Grant $K$, Gage $D$, et al. Social dominance in monkeys: dopamine $D_{2}$ receptors and cocaine self-administration. Nature Neurosc 2002;5(2):169-174.

27. Howes OD, Fusar-Poli P, Bloomfield $M$, Selvaraj S, McGuire P. From the prodrome to chronic schizophrenia: the neurobiology underlying psychotic symptoms and cognitive impairments. Curr pharmac des 2012;18(4):459-465.

28. van Nierop M, van Os J, Gunther N, et al. Does Social Defeat mediate the association between childhood trauma and psychosis? Evidence from the Nemesis-2 study. Acta Psychiat Scand 2014;129(6):467-476.

29. van Os J, Kapur S. Schizophrenia. Lancet 2009;374:635-645. 
30. van Nierop $M$, van Winkel $R$, Viechtbauer W, et al. Childhood trauma and polygenic risk for schizophrenia: no evidence for gene-environment correlation or gene-environment interaction in a family-based sample. submitted.

31. Schizophrenia Working Group of the Psychiatric Genomic Consortium. Biological insights from 108 schizophrenia-associated genetic loci. Nature 2014; in press.

32. Alemany $S$, Goldberg $X$, van Winkel R, Gasto C, Peralta V, Fananas L. Childhood adversity and psychosis: examining whether the association is due to genetic confounding using a monozygotic twin differences approach. Eur psych: j Ass Eur Psychiatr May 2013;28(4):207-212.

33. van Nierop $M$, van Dam $D$, Viechtbauer $W$, Velthorst $E$, van Winkel R, GROUP Investigators. Childhood abuse and neglect in relation to presence and persistence of psychotic and depressive symptomatology. Psychol Med 2014; doi:10.1017/S0033291714001561.

34. Glaser D. Child abuse and neglect and the brain--a review. J child psychol psychiat all discip Jan 2000;41(1):97-116.

35. Heim C, Newport DJ, Mletzko T, Miller $A H$, Nemeroff $C B$. The link between childhood trauma and depression: insights from HPA axis studies in humans. Psychoneuroendocrinology Jul 2008;33(6):693-710.

36. Wigman JTW, van Nierop $M$, Vollebergh W, Lieb R, Beesdo-Baum K, Wittchen HU, Van Os J. Evidence that psychotic symptoms are prevalent in disorders of anxiety and depression, impacting on illness onset, risk, and severity - implications for diagnosis and ultra-high risk research. Schizophr Bull 2012;38(2):247-257.

37. Hanssen $M$, Peeters $F$, Krabbendam $L$, Radstake S, Verdoux H, van Os J. How psychotic are individuals with non-psychotic disorders? Soc Psychiatry Psychiatr Epidemiol Mar 2003;38(3):149-154.

38. Olfson $M$, Lewis-Fernandez $R$, Weissman MM, Feder A, Gameroff MJ, Pilowsky D, Fuentes M. Psychotic symptoms in an urban general medicine practice. Am J Psychiat Aug 2002;159(8):1412-1419.

39. Kaymaz N, van Os J, de Graaf R, Ten Have M, Nolen W, Krabbendam L. The impact of subclinical psychosis on the transition from subclinicial mania to bipolar disorder. J Affect Disord Feb 2007;98(1-2):55-64.

40. Perlis RH, Uher R, Ostacher $\mathrm{M}$, Goldberg JF, Trivedi MH, Rush AJ, Fava M. Association between bipolar spectrum features and treatment outcomes in outpatients with major depressive disorder. Arch Gen Psychiatry Apr 2011;68(4):351-360.

41. Garety PA, Kuipers E, Fowler $D$, Freeman D, Bebbington PE. A cognitive model of the positive symptoms of psychosis. Psychol Med Feb 2001;31(2):189-195.

42. Freeman D, Garety PA. Connecting neurosis and psychosis: the direct influence of emotion on delusions and hallucinations. Behav Res Ther Aug 2003;41(8):923-947.

43. Van Rossum I, Dominguez MD, Lieb R, Wittchen HU, Van Os J. Affective dysregulation and reality distortion: a 10-year prospective study of their 
association and clinical relevance.

Schizophr Bull 2011;37(3):561-571.

44. Lichtenstein P, Yip BH, Björk C, Pawitan Y, Cannon TD, Sullivan PF, Hultman CM. Common genetic determinants of schizophrenia and bipolar disorder in Swedish families: a population-based study. Lancet 2009;373:234-239.

45. Weiser M, van Os J, Davidson M. Time for a shift in focus in schizophrenia: from narrow phenotypes to broad endophenotypes. Br J Psychiatry Sep 2005;187:203-205.

46. Mortensen PB, Pedersen MG, Pedersen CB. Psychiatric family history and schizophrenia risk in Denmark: which mental disorders are relevant? Psychol Med Feb 2010;40(2):201-210.

47. Myin-Germeys I, van Os J. Stressreactivity in psychosis: evidence for an affective pathway to psychosis. Clin Psychol Rev 2007;27:409-424.

48. van Nierop $M$, Viechtbauer $W$, Gunther N, et al. Childhood trauma is associated with a specific admixture of affective, anxiety, and psychotic symptoms cutting across traditional diagnostic boundaries. Psychol Med 2014;doi: 10.1017/ S0033291714002372.

49. van Nierop $M, B a k M$, de Graaf $R$, ten Have $M$, van Dorsselaer S, GROUP Investigators, van Winkel R. The functional and clinical relevance of childhood trauma-related admixture of affective, anxious and psychosis symptoms: further support for a stratified medicine approach in psychiatry. submitted.

50. Wheeler AL, Voineskos AN. A review of structural neuroimaging in schizop- hrenia: from connectivity to connectomics. Front hum neurosc 2014;8:653.

51. Goldberg IK. Dexamethasone suppression tests in depression and response to treatment. Lancet Jul 12 1980;2(8185):92.

52. Kapur S, Phillips AG, Insel TR. Why has it taken so long for biological psychiatry to develop clinical tests and what to do about it? Mol Psychiat 2012;17:1174-1179.

53. Familial breast cancer: collaborative reanalysis of individual data from 52 epidemiological studies including 58,209 women with breast cancer and 101,986 women without the disease. Lancet Oct 27 2001;358(9291):1389-1399.

54. Petrakos I, Kontzoglou K, Nikolopoulos TP, Papadopoulos O, Kostakis A. Glottic and supraglottic laryngeal cancer: epidemiology, treatment patterns and survival in 164 patients. J BUON Oct-Dec 2012;17(4):700-705.

55. Deng $X$, Zheng $X$, Yang $H$, Moreira JM, Brunner N, Christensen $\mathrm{H}$. Comparative Analysis of Evolutionarily Conserved Motifs of Epidermal Growth Factor Receptor 2 (HER2) Predicts Novel Potential Therapeutic Epitopes. PLoS One 2014;9(9):e106448.

56. Martin V, Cappuzzo F, Mazzucchelli L, Frattini M. HER2 in solid tumors: more than 10 years under the microscope; where are we now? Fut onc (London, England) Jun 2014;10(8):1469-1486.

57. Owen DR, Rupprecht R, Nutt DJ. Stratified medicine in psychiatry: a worrying example or new opportunity in the treatment of anxiety? J psy- 
chopharm (Oxford, England) Feb

2013;27(2):119-122.

58. Schumann G, Binder EB, Holte A, et

al. Stratified medicine for mental disorders. Eur neuropsychopharm: Jan 2014;24(1):5-50.

59. Teicher MH, Samson JA. Childhood maltreatment and psychopathology: A case for ecophenotypic variants as clinically and neurobiologically distinct subtypes. Am J Psychiat Oct 1 2013;170(10):1114-1133.

60. Szyf M. The genome- and system-wide response of DNA methylation to early life adversity and its implication on mental health. Can $j$ psychiat Revue canadienne de psychiatrie Dec 2013;58(12):697-704.

61. Rutten BP, Mill J. Epigenetic mediation of environmental influences in major psychotic disorders. Schizophr Bull Nov 2009;35(6):1045-1056.

62. Mehta $\mathrm{D}$, Klengel $\mathrm{T}$, Conneely KN, et al. Childhood maltreatment is associated with distinct genomic and epigenetic profiles in posttraumatic stress disorder. Proc Nat Ac Scie USA May 14 2013;110(20):8302-8307.

63. McGowan PO, Sasaki A, D’Alessio AC, Dymov S, Labonte B, Szyf M, Turecki G, Meaney MJ. Epigenetic regulation of the glucocorticoid receptor in human brain associates with childhood abuse. Nat Neurosci Mar 2009;12(3):342-348.

64. Perroud N, Dayer A, Piguet C, Nallet A, Favre $S$, Malafosse A, Aubry JM. Childhood maltreatment and methylation of the glucocorticoid receptor gene NR3C1 in bipolar disorder. Br J Psychiat Jan 2014;204(1):30-35.

65. Nicolaides NC, Kyratzi E, Lamprokostopoulou A, Chrousos GP,
Charmandari E. Stress, the Stress

System and the Role of

Glucocorticoids.

Neuroimmunomodulation Sep 12 2014;22(1-2):6-19.

66. Ogino $S$, Lochhead $P$, Chan AT, et al. Molecular pathological epidemiology of epigenetics: emerging integrative science to analyze environment, host, and disease. Mod path Apr 2013;26(4):465-484.

67. De Bont $P A$, van den Berg DP, van der Vleugel $B M$, et al. A multi-site single blind clinical study to compare the effects of prolonged exposure, eye movement desensitization and reprocessing and waiting list on patients with a current diagnosis of psychosis and co morbid post traumatic stress disorder: study protocol for the randomized controlled trial Treating Trauma in Psychosis. Trials 2013;14(151):doi:

10.1186/1745-6215-1114-1151.

68. de Bont PA, van Minnen A, de Jongh

A. Treating PTSD in patients with psychosis: a within-group controlled feasibility study examining the efficacy and safety of evidence-based PE and EMDR protocols. Behav ther Dec 2013;44(4):717-730.

69. Read J, Hammersley P, Rudegeair T. Why, when and how to ask about childhood abuse. Adv Psychiat Treat 2007;13:101-110. 


\section{SUMMARY}

The first part of this thesis (chapters two through seven) focused on the link between childhood trauma and psychosis, investigating possible underlying mechanisms that may explain found associations. The second part of this thesis (chapters eight through eleven) broadened the scope to other psychopathology as well, by assessing a more comprehensive psychopathological and functional outcome of experiences of childhood traumatic events.

In chapter two, the very basics of psychosis research were investigated; how psychosis is measured. In this chapter, two different, often used, measures of psychosis were utilized; a self-report measure and a more extensive clinical interview. Three main groups were compared on measures of psychosis-related characteristics: (1) individuals who did not self-report psychosis (controls), (2) individuals who did self-report psychosis, but these reports were assessed as "false-positive" after clinical interview (false-positive [FP] group), and (3) individuals who had clinically validated psychotic experiences (true positive [TP] group). For a great number of psychosis-related characteristics (such as childhood trauma, affective symptoms, and social functioning), the FP group was much more similar to the TP group than to the controls. These findings indicated that false-positive ratings on selfreport measures for psychosis carry important risk-related information, and may represent the mildest expression of psychosis.

In chapter three, factors associated with a transition from health to psychotic disorder were investigated. This study differed from traditional ultra-high risk studies, as unaffected siblings of patients with a psychotic disorder (elevated genetic risk for psychosis), and (non-psychotic) controls (average genetic risk for psychosis) were included in this study, instead of individuals at ultra-high risk for psychosis (i.e., help-seeking individuals). This study uncovered multiple common environmental risk factors associated with transition, such as minority status, urban birth, cannabis use, and (with the strongest association) childhood trauma. Most of the individuals in this sample were exposed to at least one environmental risk factor, thus the impact of these factors can only be detected against the background of differential genetic risk, indicating gene-environment interaction.

Five existing psychological models for the association between childhood trauma and psychosis were investigated in chapter four. No support was found for the notion that childhood trauma shows a stronger association with hallucinations than with delusions, nor that particular types of trauma show a stronger association with auditory-verbal hallucinations than with paranoia. No evidence was found that abuse shows a stronger association with psychosis than neglect. This study did find evidence that exposure to trauma is associated with a greater likelihood of co-occurring hallucinations and delusions, compared with either symptoms in isolation. In addition, traumatic experiences involving a perpetrator with an intention to harm (i.e., abuse) showed a stronger association with psychosis than trauma without intent (death of a loved one). 
Chapter five reviewed the available evidence of the link between childhood trauma and psychosis, highlighting the social defeat hypothesis of psychosis. Several known risk factors for psychosis, such as growing up in an urban environment, hearing impairment, low IQ, minority status, and childhood trauma, can all lead to a state of social defeat through abnormailities in dopaminergic neurotransmission. This, in turn, can ultimately lead to psychosis.

This theory is investigated further in chapter six. It was found that the association between trauma and psychosis was indeed mediated by feelings fitting a state of social defeat, such as loss of confidence, and hopelessness.

In chapter seven, a state-of-the-art quantitative genetic measure, i.e., the polygenic risk score, was used to assess it's association with psychosis and with childhood trauma. The association with psychosis was replicated, however, no interaction with childhood trauma was found. Moreover, evidence against gene-environment correlation was reported, as childhood trauma was not associated with polygenic risk, nor was the association between trauma and psychosis significantly influenced (confounded) by polygenic risk.

In chapter eight, the link between abuse and neglect on the one hand, and symptoms of psychosis and depression on the other, was investigated. The main findings were that childhood trauma did not show a stronger association with psychosis, than with depression, even in a sample of patients with a psychotic disorder, or their unaffected siblings (at elevated genetic risk for psychosis). Moreover, psychosis symptom severity decreased in a similar rate over the follow-up period of three years, when comparing traumatized individuals to their non-traumatized counterparts.

Patients with an anxiety or depressive disorder were investigated in chapter nine, comparing those patients who also reported psychotic symptoms to patients without psychotic symptoms. It was found that patients with psychotic symptoms displayed an increased illness severity, more help seeking behaviour, and more often had a history of childhood trauma, than patients without psychotic symptoms. These findings indicated that not only is the co-occurrence of psychotic symptoms in such patient populations not rare $(27 \%)$, but also very clinically relevant.

Thus, in chapter ten, possible co-occurrence of symptoms of depression, anxiety, psychosis, and mania was investigated, in the general population, and in samples of patients with a mood, anxiety, or psychotic disorder. It was found that as more (frequent) childhood trauma was experienced, more symptom clusters (combinations of two, three, or four symptom clusters) were reported, in all samples. In chapter eleven, these patient samples were investigated in terms of social and clinical functioning, contrasting groups with or without trauma history, and with or without clustering of symptoms. It was found that in particular the group with a trauma history, and co-occurring symptoms showed a poorer functional outcome, compared with patients without a trauma history, without co-occurrence of symptoms, or neither. Based on these findings, the benefits of using a stratified approach in psychiatry (stratifying according to trauma history) was argued. 


\section{SAMENVATTING}

In het eerste deel van deze thesis (hoofdstuk twee tot en met zeven) lag de nadruk op de associatie tussen jeugdtrauma en psychose, waarbij mogelijke onderliggende mechanismen werden onderzocht. In het tweede deel (hoofdstuk acht tot en met elf) werd er ook naar andere psychopathologie gekeken, door uitgebreider de psychopathologische en functionele consequenties van traumatische ervaringen in de jeugd te onderzoeken.

In hoofdstuk twee werd de basis van psychose onderzoek geëxploreerd; hoe psychose wordt gemeten. In dit hoofdstuk werden twee verschillende, vaak gebruikte, methodes om psychose te meten toegepast; zelfrapportage en een meer uitgebreid klinisch interview. Drie hoofdgroepen werden vergeleken op verschillende psychose-gerelateerde karakteristieken: (1) mensen die geen psychose zelf-rapporteerden (controles), (2) mensen die wel psychose zelf-rapporteerden, maar waarbij na klinisch interview werd besloten dat deze rapportages "fout-positief" waren (fout-positief [FP] groep), en (3) mensen die klinisch gevalideerde psychotische ervaringen hebben gehad (echt-positief [EP] groep). Voor een groot aantal psychose-gerelateerde karakteristieken (zoals jeugdtrauma, affectieve symptomen, en sociaal functioneren), was de FP groep meer overeenkomstig met de EP groep, dan met de controles. Deze bevindingen laten zien dat fout-positieven op zelfrapportages voor psychose belangrijker risico-gerelateerde informatie bevatten, en wellicht de mildste uiting van psychose vertegenwoordigen.

In hoofdstuk drie werden factoren die geassocieerd zijn met transitie van mentale gezondheid naar een psychotische stoornis onderzocht. Deze studie verschilde van traditionele 'Ultra-High Risk' studies, omdat de (niet psychotische) broers en zussen van patiënten met een psychotische stoornis (dus met verhoogd genetisch risico voor psychose) en (niet psychotische) controles (gemiddeld genetische risico) werden geïncludeerd in deze studie, in plaats van mensen met 'ultra-high risk' (dus hulpzoekende individuen). In deze studie werden meerdere omgevingsfactoren gevonden die geassocieerd waren met transitie, zoals etnische minderheidsstatus, geboren worden in stad, cannabis gebruik, en (met als sterkste associatie) jeugdtrauma. De meeste van de deelnemers waren blootgesteld aan minstens één van deze omgevingsfactoren, dus het effect hiervan kan alleen gemeten worden tegen de achtergrond van verschillende niveaus van genetisch risico, iets dat op gen-omgevingsinteractie wijst.

Vijf bestaand psychologische modellen voor de associatie tussen jeugdtrauma en psychose werden onderzocht in hoofdstuk vier. Er werd geen bewijs gevonden dat jeugdtrauma een sterkere associatie heeft met hallucinaties dan met wanen, noch dat bepaalde soorten trauma's sterker geassocieerd zijn met auditief-verbale hallucinaties dan met paranoïde wanen. Ook werd er geen bewijs gevonden dat mishandeling sterker geassocieerd is met psychose dan verwaarlozing. Deze studie vond wel aanwijzingen dat blootstelling aan trauma sterker geassocieerd is met het hebben van wanen en hallucinaties samen, vergeleken met het hebben van alléén hallucinaties of alléén wanen. Bovendien lieten die soorten trauma waarbij er sprake was van een dader met intentie (zoals mishandeling) een sterker effect zien dan trauma zonder intentie (zoals het overlijden van een naaste). 
Hoofdstuk vijf gaf een overzicht van studies naar de link tussen jeugdtrauma en psychose, waarbij de 'social defeat' hypothese in het bijzonder werd besproken. Meerdere bekende risicofactoren voor psychose, zoals opgroeien in de stad, gehoor problemen, een laag IQ, lid zijn van een minderheidsgroep, en jeugdtrauma, kunnen allemaal door veranderingen in de dompaminerge neurotransmissie leiden tot een staat van 'social defeat'. Dit laatste kan dan weer leiden tot psychose.

Deze theorie wordt verder onderzocht in hoofdstuk zes. Hier werd gevonden dat de associatie tussen jeudtrauma en psychose werd gemedieerd door symptomen passend bij 'social defeat', zoals een laag zelfbeeld, en gevoelens van hopeloosheid.

In hoofdstuk zeven werd een nieuwe kwantitatieve genetisch methode, de 'polygenic risk score', gebruikt om te kijken in hoeverre die geassocieerd is met psychose en jeugdtrauma. Allereerst was de polygenic risk score geassocieerd met psychose, maar er werd geen interactie met jeugdtrauma gevonden. Bovendien werd er bewijs tegen gen-omgevings correlatie gevonden, omdat jeugdtrauma niet geassocieerd was met polygenic risk, en de associatie tussen trauma en psychose was niet significant beïnvloed door polygenic risk.

In hoofdstuk acht werd de link tussen mishandeling en verwaarlozing, en psychotische en depressieve symptomen onderzocht. De hoofdbevindingen waren dat jeugdtrauma niet een sterkere associatie liet zien met psychose dan met depressie, zelfs in een groep van mensen met een psychotische stoornis, of hun broers of zussen (met verhoogd genetisch risico voor psychose). Verder werd daalde de ernst van de psychose symptomen met dezelfde hoeveelheid bij mensen die jeugdtrauma hebben meegemaakt, vergeleken met mensen die geen trauma hebben meegemaakt.

Patiënten met een angststoornis of een depressieve stoornis werden onderzocht in hoofdstuk negen. Hierin werden die patiënten die ook psychotische symptomen rapporteerden vergeleken met patiënten zonder psychotische symptomen. In dit hoofdstuk werd gevonden dat patiënten met psychotische symptomen een grotere ziekte-ernst hadden, vaker hulp zochten, en vaker jeugdtrauma hadden meegemaakt. Deze bevindingen lieten zien dat psychotische symptomen vaak voorkomen bij mensen met een angststoornis of een depressieve stoornis (27\%), maar ook dat dit fenomeen erg klinisch relevant is.

Daarom werd in hoofdstuk tien de comorbiditeit van depressieve, angst, psychotische en manie symptomen onderzocht, in een algemene bevolkingsstudie, en in groepen patiënten met een stemmings-, angst-, of psychotische stoornis. Er werd gevonden dat wanneer er meer (frequenter) jeugdtrauma was meegemaakt, er meer symptoom clusters (combinatie van twee, drie, of vier symptoom clusters) werden gerapporteerd. In hoofdstuk elf werden deze patiënt groepen onderzocht naar hun sociale en klinisch functioneren, waarbij de groepen werden gecontrasteerd naar trauma geschiedenis en symptoom clustering. Hieruit bleek dat vooral de groep patiënten met een trauma geschiedenis en meerdere symptoom clusters slechter functioneerden dan patiënten zonder trauma geschiedenis, en/of zonder meerdere symptoom clusters. Deze bevindingen laten de toegevoegde waarde zien van stratificeren (naar trauma geschiedenis) binnen de psychiatrie. 


\section{VALORIZATION}

The cost of mental disorders is staggering. In Europe, in 2010 alone, these costs have been estimated at 113.4 billion euro for mood disorders, 74.4 billion euro for anxiety disorders, and 93.9 billion euro for psychotic disorders ${ }^{1}$. These costs constitute the direct health care costs, direct non-medical costs (such as social services or special accommodation), but also indirect costs due to work absence or early retirement. In mental disorders, these indirect costs form the largest portion of the total costs (48\%), thus patients with a mental disorder who display poor functioning (e.g. not able to work) put the highest strain on healthcare expenditure ${ }^{1}$. Of course, these numbers do not express the personal suffering these diseases can cause in patients with psychiatric disorders, or their families and friends. Arguably, this strain is also highest when disease severity is high, quality of life is poor, and social or vocational functioning is low, both in the patients as well as in their relatives and friends ${ }^{2}$.

Thus, new avenues for more effective treatment options are urgently needed. Currently, following examples from oncology, researchers are arguing for the implementation of personalized medicine in psychiatry ${ }^{3}$. This mostly entails assessing genetic or epigenetic factors associated with a given mental disorder, guiding pharmacologic therapies (i.e. estimating the choice and dosage of medication based on genetic profiles) ${ }^{3}$. Studies providing clinically applicable evidence, however, are sparse and in their infancy. The findings from this thesis provide a practical alternative, by stratifying according to childhood trauma history. Patients with a mental disorder (anxiety disorder, depression, or psychotic disorder) who had experienced childhood traumatic events displayed poorer functioning (social or vocational functioning, or quality of life) than patients without a trauma history, especially when these traumatized patients also displayed a mixed phenotype of anxious, affective, manic, and psychotic symptoms. Therefore, patients with a trauma history may be more resistant to the treatment programs currently available. This shows that in particular these patients with a trauma history arguably put the highest strain on healthcare costs, have the greatest personal suffering, and put the greatest strain on their loved ones. Therefore, by stratifying according to trauma history, instead of focusing on diagnostic categories, important within group differences are explained. Screening for childhood trauma history can be fast and cost-effective, will increase prognostic values, and can provide healthcare professionals with important needs predictions early in the treatment process. Furthermore, these findings would argue that implementing trauma-treatment in patients with common mental disorder (i.e. anxiety disorder or depression) or psychotic disorder and with a childhood trauma history (thus implementing personalized medicine), could prove very beneficial in reducing disease severity, improving functional outcome, and therefore also reducing direct or indirect health care costs. These types of treatments, however, such as trauma-focused cognitive behavioural therapy or eye movement desensitization (EMDR), are currently only offered to patients with posttraumatic stress disorder (PTSD). In one study, recently initiated, psychotic disorder patients with comorbid PTSD showed a decrease in general psychopathology (aside from improvement of PTSD symptoms) after trauma-treatment ${ }^{4}$. 
Further studies are needed to investigate whether these promising results may also translate to psychiatric patients without PTSD.

However, prevention of childhood trauma would of course be much more effective. Although some work is being done for primary prevention, such as national add campaigns against child abuse or school programs designed to prevent bullying, childhood trauma is highly prevalent, and the number of instances of child abuse has not gone down in recent years 5 . In the Netherlands, 118,000 children (0-18 years) have been exposed to some type of abuse (by an adult) in 2010 alone $^{5}$. Additionally, over $10 \%$ of school-aged children report feeling unsafe in school due to bullying ${ }^{6}$. By reporting on the detrimental outcome of childhood trauma, using rigorous analyses in large and representative samples, this thesis provides important additional awareness, which may convince municipal and national governments to increase efforts for primary prevention. Furthermore, this thesis reports evidence arguing strongly for a causal association between childhood trauma and psychopathology (i.e. evidence against gene-environment correlation, and evidence for plausible underlying mechanisms), increasing its persuasiveness.

Findings from this thesis have been and will be presented at conferences aimed at healthcare professionals, working in adult and children's (mental or somatic) healthcare, as well as published in international and national scientific journals. This will underscore the importance of early detection with young adults, increasing efforts for secondary prevention, as arguably any trauma-related treatment will be much more effective when applied immediately rather that decades later. Additionally, this will hopefully induce healthcare professionals to include trauma assessment in the standard protocol for adult psychiatric patients, which currently is far from daily practice ${ }^{7}$. When patients present with mental problems (but not PTSD symptoms), and do not spontaneously report traumatic events from their childhood, health care professionals tend not to ask about a possible trauma history ${ }^{7}$. Moreover, patients may not want to talk about these painful events, or are less aware of how these events may still have a profound effect in adulthood. Thus, these findings have also been presented at conferences and published in magazines aimed at service users, thus increasing awareness amongst patients with a mental disorder. By doing so, service users may also be encouraged to initiate a discussion about their early experiences with their care providers.

From a scientific perspective, this thesis adds to the body of work stressing the importance of childhood traumatic experiences in the development of mental health problems, after years of ignoring it as a valid risk factor, or even dismissing reports of abusive events as fantasy 8 . Additionally, these findings provide important additional fuel to the debate of possible shared pathways or vulnerabilities across diagnostic categories. By showing that childhood trauma is similarly associated with psychotic as well as depressive symptoms in a sample of patients with a psychotic disorder or their unaffected siblings, and showing similar phenotypic and functional outcome of trauma across diagnostic categories, evidence of a shared etiological pathway has been provided. Combining these findings with findings from epigenetic studies can form the basis of a new direction in psychiatric research; decreasing the focus on traditional diagnostic categories (top-down approach), 
and greatly increasing the focus on etiological mechanisms (bottom-up approach). Given the relative lag of psychiatric medicine compared with somatic medicine, evidence provided in this thesis concerning adopting a stratified approach could be an important step towards decreasing this gap. Only by getting a firm grasp on the etiology of psychiatric disorders, arguably through environmental stratification, true progress in understanding the mechanisms of mental illness can be obtained.

This thesis also provides important evidence towards the necessity of applying suitable statistical techniques when reporting on etiological models based on specificity. For example: only by applying mixed-effects multilevel models utilized in this thesis it was revealed that no specific effects (e.g. association of childhood trauma with delusions versus hallucinations) were present, even when one effect size was significant and the other was not. Through publications and presentations at scientific conferences, several requests have already been made for assistance in implementing these models. By including large and diverse samples (representative of the general population, healthy samples, samples of patients with a mood disorder, patients with an anxiety disorder, patients with a psychotic disorder and their unaffected siblings; thus increasing generalizability), important insights into mechanisms of childhood trauma and (subclinical) psychopathology have been revealed. 


\section{REFERENCES}

1. Gustavsson A, Svensson M, Jacobi F, et al. Cost of disorders of the brain in Europe 2010. European neuropsychopharmacology : the journal of the European College of Neuropsychopharmacology 2011;21(10):718-779.

2. Goncalves-Pereira $M$, Xavier $M$, van Wijngaarden B, Papoila AL, Schene AH, Caldas-de-Almeida JM. Impact of psychosis on Portuguese caregivers: a cross-cultural exploration of burden, distress, positive aspects and clinicalfunctional correlates. Soc Psychiatry Psychiatr Epidemiol Feb 2013;48(2):325-335.

3. Ozomaro U, Wahlestedt C, Nemeroff $\mathrm{CB}$. Personalized medicine in psychiatry: problems and promises. BMC medicine 2013;11:132.

4. de Bont PA, van Minnen A, de Jongh A. Treating PTSD in patients with psychosis: a within-group controlled feasibility study examining the efficacy and safety of evidence-based PE and EMDR protocols. Behavior therapy Dec 2013;44(4):717-730.
5. Kindermishandeling. Available at: http://www.nji.nl/ Kindermishandeling. Accessed Oct 10, 2014.

6. Cijfers over pesten. Available at: http://www.nji.nl/Cijfers-over-pesten. Accessed Oct 10, 2014.

7. Read J, Hammersley P, Rudegeair T. Why, when and how to ask about childhood abuse. Advances in Psychiatric Treatment 2007;13:101-110.

8. Read J, Bentall RP. Negative childhood experiences and mental health: theoretical, clinical and primary prevention implications. $\mathrm{Br} \mathrm{J}$ Psychiatry Feb 2012;200(2):89-91. 


\section{DANKWOORD}

Ik had deze thesis nooit alleen tot stand kunnen brengen, en wil daarom graag de volgende mensen nog bedanken:

Allereerst grote dank aan alle deelnemers van GROUP en NEMESIS, zonder jullie bijdrage was (onder andere) deze thesis er niet geweest!

En dan natuurlijk mijn promotie dreamteam. Jim en Inez, jullie beide als promotors krijgen was op zich al heel bijzonder, maar de kansen die jullie mij hebben geboden en deuren die overal spontaan voor mij open zijn gegaan waren echt onmisbaar. De input die ik van jullie allebei heb gekregen, zeker omdat dit van zo verschillende achtergronden vandaan kwam, heeft ervoor gezorgd dat ik een zo breed mogelijke ontwikkeling kon maken. Jullie boden mij door jullie expertise en vertrouwen in mijn kunnen de steigers die ik nodig had om mijn carrière te beginnen. Maar vooral wil ik Ruud heeeeel erg bedanken. Je stond letterlijk elke dag voor me klaar om me overal mee te helpen. Jij dwong me het grotere plaatje te zien, als ik weer eens bijna verdronk in de gigantische berg data die ik op mijn schoot geworpen had gekregen. Door jou heb ik sommige inzichten gekregen waar ik in mijn eentje nooit op was gekomen. Mijn zelfvertrouwen in wat ik qua werk aankan is enorm gegroeid, en dat is voor het grootste deel toe te schrijven aan de manier waarop jij mij hebt begeleid. Als ik het even niet meer zag zitten, wist jij me feilloos uit de put te trekken. Ik hoop dat ik net zo'n goede begeleider en wetenschapper kan worden als jij.

De hele afdeling heb ik eigenlijk altijd als heel behulpzaam en gezellig ervaren, dus allemaal heel erg bedankt! Mayke en Sanne, voor m'n gevoel hebben we dit echt samen gedaan en meegemaakt, wat me heel erg heeft geholpen om alles voor elkaar te krijgen (gedeelde smart en zo). En door jullie waren de congressen ook erg gezellig! Christine en Aleida, laten we nog eens teruggaan naar Florence, maar dan als ik niet een praatje hoef te houden.. Dennis en Nicole L., volgens mij heb ik nooit helemaal begrepen wat jullie nou deden, maar jullie waren altijd in voor een gezellig praatje, en altijd bereid te helpen. Catherine, ik heb zoveel geleerd door mee te werken aan jouw stigma project, en wat een eer dat ik jouw paranimf mocht zijn! Wolfgang, dankzij al jouw geduldige uitleg denken andere mensen nu dat ik goed in statistiek ben.. ;-) Marjan, ook erg bedankt voor al je onmisbare statistiek en Stata hulp! Maarten, dank voor je hulp bij NEMESIS, je humor, en input op mijn papers. Truda, je was mijn inval-moeder van de afdeling, wat moet ik straks zonder jou?! Debora, ook erg bedankt voor jouw inval-Truda hulp. Wendy en Karin, volgens mij worden jullie überhaupt niet genoeg bedankt voor wat jullie allemaal doen, iets wat ik vooral heb geleerd na de Op de Pedalen dag, dus bij deze! Ron, jij stond ook altijd voor me klaar, volgens mij heb je zelfs ook nog eens mijn telefoon onder handen genomen.. En natuurlijk de Nele's, bedankt voor alle hulp met Geestkracht! Roos en Silke, jullie hebben me alles geleerd over interviews en NPO's doen, en jullie waren vooral hele gezellige instructeurs. Tineke, Dina en Marieke, dank voor alle advies, jullie waren belangrijke voorbeelden voor me! Petra en Anne, heel erg bedankt voor het doen van al die NEMESIS interviews! En voor alle 'verse' AIO's: veel succes! Ik vond het erg leuk om jullie te leren kennen (met een speciale dank aan Yori voor het overnemen van de GROUP data). Feikje, dank voor al je hulp, en voor ons paper! 
Rufa, ik ben zo blij dat ik jou hier heb leren kennen! Ik vond het zo ontzettend jammer toen je wegging bij UM, maar gelukkig heeft dat niets veranderd aan onze relatie. Ik kon altijd alles aan jou kwijt, en voel me vereerd dat jij mijn paranimf wilde zijn. Net als Claudia eigenlijk! Vier jaar hebben we naast elkaar gezeten, en onze (behoorlijk verschillende) levens gedeeld. In de tussentijd ben jij zelfs een getrouwde vrouw en moeder geworden, en dan krijg je het ook nog eens voor elkaar te promoveren! Jij was mijn klankbord, mijn raadgever, en vooral een ontzettend goede luisteraar.

Ron de Graaf, Margreet ten Have, en Saskia van Dorsselaer: NEMESIS is een heel groot gedeelte van mijn PhD, echt heel erg bedankt dat ik jullie geweldige data mocht gebruiken (hoeveel mensen kunnen een $n=13.722$ studie in hun thesis zetten..?), en bedankt voor alle input op mijn papers!

Craig Morgan, thank you for letting me stay at your department, and using your data (which in the end unfortunately didn't make it to the thesis, but nonetheless will turn into a nice paper eventually!) and expertise.

Grote dank aan alle GROUP Investigators (Richard Bruggeman, Wiepke Cahn, Lieuwe de Haan, Rene Kahn, Carin Meijer en Durk Wiersma) voor alle onmisbare input op mijn GROUP papers. Daniella van Dam, bedankt voor de leuke en vruchtbare samenwerking op ons paper! And thanks to the Psychiatric Genomic Consortium for the polygenic risk scores.

En dan natuurlijk de harde kern van mijn familie. Mama, je zal je misschien tijdens mijn studie wel eens op je hoofd gekrabd hebben of er nog wel wat van terecht zou komen. Gelukkig dus uiteindelijk wel, en voor een groot deel omdat jij me altijd hebt bijgestaan. Ook niet onbelangrijk is dat jij me altijd hebt geleerd dat ik moet doen wat mij gelukkig maakt, en niet wat andere mensen misschien van me verwachten. (en sorry, waarschijnlijk dus geen kleinkinderen voor jou van mijn kant.. ;-)) Ik kan eigenlijk bijna precies hetzelfde zeggen over Petra. Er is denk ik niemand die me zo goed kent als jij, en niet een beter iemand die ik als voorbeeld kon hebben. Hoewel we toch wel wat jaren schelen, en we niet zo lang in één huis hebben gewoond, is onze band altijd even sterk geweest. Wouter, jij staat ook altijd voor me klaar, of het nu om de duizendste computer-vraag gaat, of weer een verhuizing. Zelfs toen we vroeger nog oorlog hadden over wie over wiens helft van de achterbank kwam op weg naar Frankrijk, was jij altijd nog mijn beschermende grote broer. Wim, ik ben zo blij dat mama zo'n lieve man als jij heeft leren kennen. Je zegt misschien niet zo heel veel, maar als je wel iets zegt zijn dat altijd zulke oprechte en lieve dingen! Ik kijk uit naar het Italiaanse volkslied over twee jaar..? And Allan, I never thought that Petra would ever get married, until I met you. You make her so happy, which makes me so happy. You're the best brother in law I could hope for.

Faye, hoe lang kennen we elkaar nu al? (pfff wat worden we oud) Op veel gebieden zo anders (bv in dimensie, en stress-levels?), maar toch ben je al zo lang mijn beste vriendin, hoe cheesy dat ook klinkt. Jij wist alle familie-, vriendjes en studie/werk drama's feilloos te relativeren. Jelle, bedankt dat je Faye zo gelukkig maakt, en voor je werk-kleptomanie.. :-) 
Barbara, Ladi, Jonathan, Alex, Joao, Bert, Mauricio and Juan: you are indeed my urban family, even though I've never actually lived in the same city as any of you.. You all taught me that life after 30 just becomes more fun, that you can have an awesome career and still have a great social life, and that Easter is actually a pretty cool holiday.

En Tom, tja, sta je er toch in.. :-) Je was en bent nog steeds een grote verrassing. Mijn promotie zal in elk geval ook voor jou niet een eitje zijn geweest. Bedankt voor al je steun, en geduld! 


\section{CURRICULUM VITAE}

Martine van Nierop was born on July $25^{\text {th }} 1982$, in The Hague, the Netherlands. After finishing secondary school at Gymnasium Haganum in The Hague, she started Higher Laboratory School in Leiden, but changed to Psychology at the University of Leiden in 2001. In 2008, she received a Bachelors of Science in Psychology. As part of her Bachelors, she worked as a research assistant on a project investigating the cognitive consequences of recreational cocaine use. In 2009, she started a Masters in Clinical Neuropsychology, also at the University of Leiden. During her Masters she worked as a research intern at Parnassia in The Hague, on a project investigating cognition and memory impairment after Electro Convulsive Therapy. Her Masters thesis involved the study into the utility of a questionnaire designed to distinguish between cognitive impairment as a result of depression and dementia. After receiving her Masters of Science degree in 2010, she was appointed as a PhD student at Maastricht University, at the department of Psychiatry and Psychology, school of Mental Health and Neuroscience. During her PhD, she was involved in several projects, such as the Genetic Risk and Outcome of Psychosis (GROUP) project and the second Netherlands Mental Health Survey and Incidence Study (NEMESIS-2), and she taught at the Faculty of Health, Medicine and Life Sciences. In 2014, she worked at the Institute of Psychiatry at King's College London, for a period of three months. 


\section{LIST OF PUBLICATIONS}

1. Van Nierop, M., van Os, J. Gunther, N., Myin-Germeys, I., de Graaf, R., ten Have, M., van Dorsselaer, S., Bak, M., van Winkel, R. (2012). Phenotypically continuous with clinical psychosis, discontinuous in need for care: evidence for an extended psychosis phenotype. Schizoprenia Bulletin 38(2) 231-238. Impact Factor (IF) 8.486

2. Van Nierop, M., Janssens, M., GROUP investigators (2013). Evidence that transition from health to psychotic disorder can be traced to semi-ubiquitous environmental effects operating against background genetic risk. PLOS ONE, 8(11), e76690. IF 3.73

3. Van Nierop, M., Lataster, T., Smeets, F., Gunther, N., van Zelst, C., de Graaf, R., ten Have, M., van Dorsselaer, S., Bak, M., Myin-Germeys, I., Viechtbauer, W., van Os, J., van Winkel, R. Psychopathological mechanisms linking childhood traumatic experiences to risk of psychotic symptoms. Analysis of a large, representative population-based sample. Schizophrenia Bulletin, 40, Suppl 2, S123-30. IF 8.486

4. Van Winkel, R., van Nierop, M., Myin-Germeys, I., van Os, J. (2013). Childhood trauma as a cause of psychosis: linking genes, psychology, and biology. Canadian Journal of Psychiatry, 58(1) 44-51. IF 2.483

5. Van Nierop, M., van Os, J., Gunther, N., van Zelst, C., de Graaf, R., ten Have, M., van Dorsselaer, S., Bak, M., Myin-Germeys, I., van Winkel R. (2014). Does Social Defeat Mediate the Association Between Childhood Trauma and Psychosis? Evidence from the Nemesis-2 Study. Acta Psychiatrica Scandinavica, 129(6) 467-476. IF 4.857

6. Van Nierop, M., van Winkel, R., Viechtbauer, W., Rutten, B., Vinckers, C., Richards, A., Owens, M., Holmans, P., O’Donovan, M., The Psychiatric Genomic Consortium \& GROUP Investigators (submitted). Childhood trauma and polygenic risk for schizophrenia: no evidence for gene-environment correlation of gene-environment interaction in a family-based sample.

7. Van Nierop, M., van Dam, D.S., Viechtbauer, W., Velthorst, E., van Winkel, R. \& GROUP investigators. Childhood abuse and neglect in relation to the presence and persistence of psychotic and depressive symptomatology. Psychological Medicine. doi:10.1017/ S0033291714001561. IF 5.587

8. Wigman, J.T., van Nierop, M., Vollebergh, W.A., Lieb, R., Beesdo-Baum, K., Wittchen, H.U., van Os, J. (2012). Evidence that psychotic symptoms are prevalent in disorders of anxiety and depression, impacting on illness onset, risk, and severity--implications for diagnosis and ultra-high risk research. Schizophrenia Bulletin, 38(2) 247-257. IF 8.486

9. Van Nierop, M., Viechtbauer, W., Gunther, N., van Zelst, C., de Graaf, R., ten Have, M., van Dorsselaer, S., Bak, M., GROUP investigators \& van Winkel, R. (2014). Childhood trauma is associated with a specific admixture of affective, anxiety, and psychotic symptoms cutting across traditional diagnostic boundaries. Psychological Medicine, doi: $10.1017 /$ S0033291714002372. IF 5.587

10. Van Nierop, M., Bak, M., de Graaf, R., ten Have, M., van Dorsselaer, S., GROUP Investigators \& van Winkel, R. (submitted). The functional and clinical relevance of childhood trauma-related admixture of affective, anxious and psychosis symptoms: further support for a stratified medicine approach in psychiatry. 
11. Van Zelst, C., van Nierop, M., Oorschot, M., Myin-Germeys, I., van Os, J., Delespaul, P.; for GROUP (2014). Stereotype awareness, self-esteem and psychopathology in people with psychosis. PLoS One, 11(2): e88586. IF 3.73

12. Van Nierop, M. Psychopathologische mechanismen van jeugdtrauma. Tijdschrift voor Psychiatrie - Nederlands-Vlaams Toponderzoek, 56(2014) 7, 477.

13. Van Zelst, C., van Nierop, M., van Dam, D., Bartels-Velthuis, A., Delespaul, P., and GROUP-investigators (submitted). Associations between stereotype awareness, childhood trauma and psychopathology: A study in people with psychosis, their siblings and controls.

14. Guloksuz, S., van Nierop, M., Lieb, R., van Winkel, R., Wittchen, H.U. \& van Os, J. (submitted). Evidence that environmental stratification in affective disorders predicts psychosis expression and helpseeking, mediated by severity of non-psychotic psychopathology.

15. van Winkel, R., Rutten, B., Richards, A., Viechtbauer, W., Wolters, K., van Nierop, M., Drukker, M., Owen, M., Holmans, P., O'Donovan, M., The Psychiatric Genomic Consortium \& GROUP Investigators (submitted). Evidence that a causal effect of cannabis on the development of psychosis is most prominent in individuals at lower polygenic risk for schizophrenia.

16. Lataster, T., Viechtbauer, W., Oorschot, M., Collip, D., Janssens, M., van Nierop, M., Menne-Lothmann, C., Lardinois, M., Lataster, J., Jacobs, N., Thewissen, V., van der Steen, Y., Wagner, M., Gimpel-Drees, J., Geschwind, N., Simons, C., Nicolson, N., Delespaul, P., Wichers, M., Myin-Germeys, I. (submitted). Evidence for a two-factor positive and negative affect structure in daily life: presenting the Maastricht Momentary Mood Questionnaire (3MQ). 